\title{
Polarity-Reversed Addition of Enol Ethers to Imines under Visible- Light: Redox-Neutral Access to Azide-Containing Amino Acids
}

\section{Sen Yang, Shuangyu Zhu, Dengfu Lu* and Yuefa Gong}

School of Chemistry and Chemical Engineering, Huazhong University of Science and Technology 1037 Luoyu Rd., Wuhan, Hubei, 430074, China

E-mails: dlu@hust.edu.cn

\section{Table of Contents}

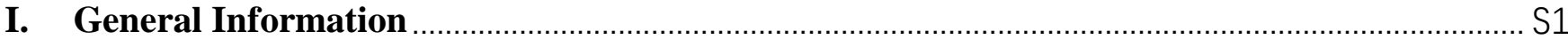

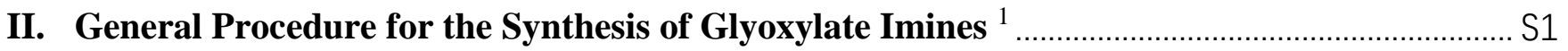

III. Condition Optimization of the Three-Component Reaction........................................................ S2

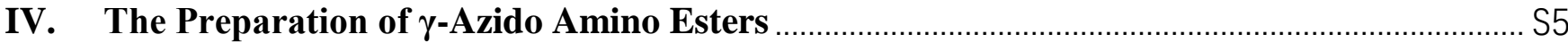

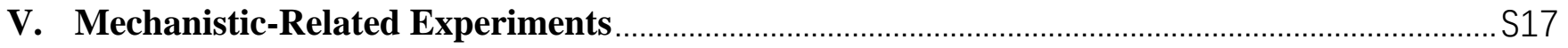

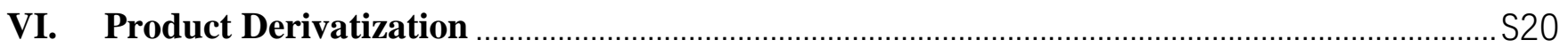

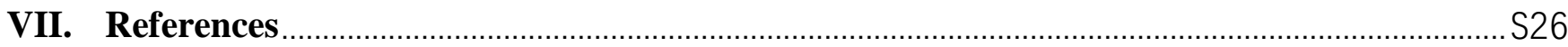

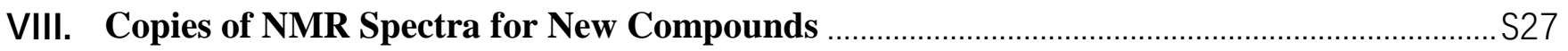




\section{General Information}

General procedures. All reactions were performed in oven-dried or flame-dried round-bottom flasks and vials. Reactions were monitored with thin layer chromatography (TLC) using silica gel 60 F-254 plates. TLC plates were normally visualized by UV irradiation ( $254 \mathrm{~nm}$ or $365 \mathrm{~nm}$ ), stained with basic $\mathrm{KMnO}_{4}$ or phosphomolybdic acid. Flash chromatography was performed using silica gel 60 (200-300 mesh).

Instrumentation. Proton nuclear magnetic resonance $\left({ }^{1} \mathrm{H}\right.$ NMR) spectra and carbon nuclear magnetic resonance $\left({ }^{13} \mathrm{C}\right.$ NMR) spectra were recorded on Bruker Ascend $400 \mathrm{MHz}$ and $600 \mathrm{MHz}$. Chemical shifts for protons are reported in parts per million downfield from tetramethylsilane and are referenced to the NMR solvent residual peak $\left(\mathrm{CHCl}_{3}: \delta 7.26\right)$. Chemical shifts for carbons are reported in parts per million downfield from tetramethylsilane and are referenced to the carbon resonances of the NMR solvent (Chloroform- $d: \delta 77.0$ ). Data are represented as follows: chemical shift, multiplicity ( $\mathrm{s}=$ singlet, $\mathrm{d}=$ doublet, $\mathrm{t}=$ triplet, $\mathrm{q}=$ quartet, $\mathrm{m}=$ multiplet, $\mathrm{br}=$ broad $)$, coupling constants in Hertz $(\mathrm{Hz})$, and integration. When ${ }^{19} \mathrm{~F}$ NMR is used for quantitative purpose (yield and $d . r$. determination), a 30-degree pulse and longer delay time $(\mathrm{d} 1=5 \mathrm{~s})$ were employed and the receiver gain was manually set as 32 . IR spectra were recorded on a Bruker FT-IR spectrometer. HRMS was measured on a TOF-Q mass spectrometer equipped with an ESI source.

\section{General Procedure for the Synthesis of Glyoxylate Imines ${ }^{1}$}
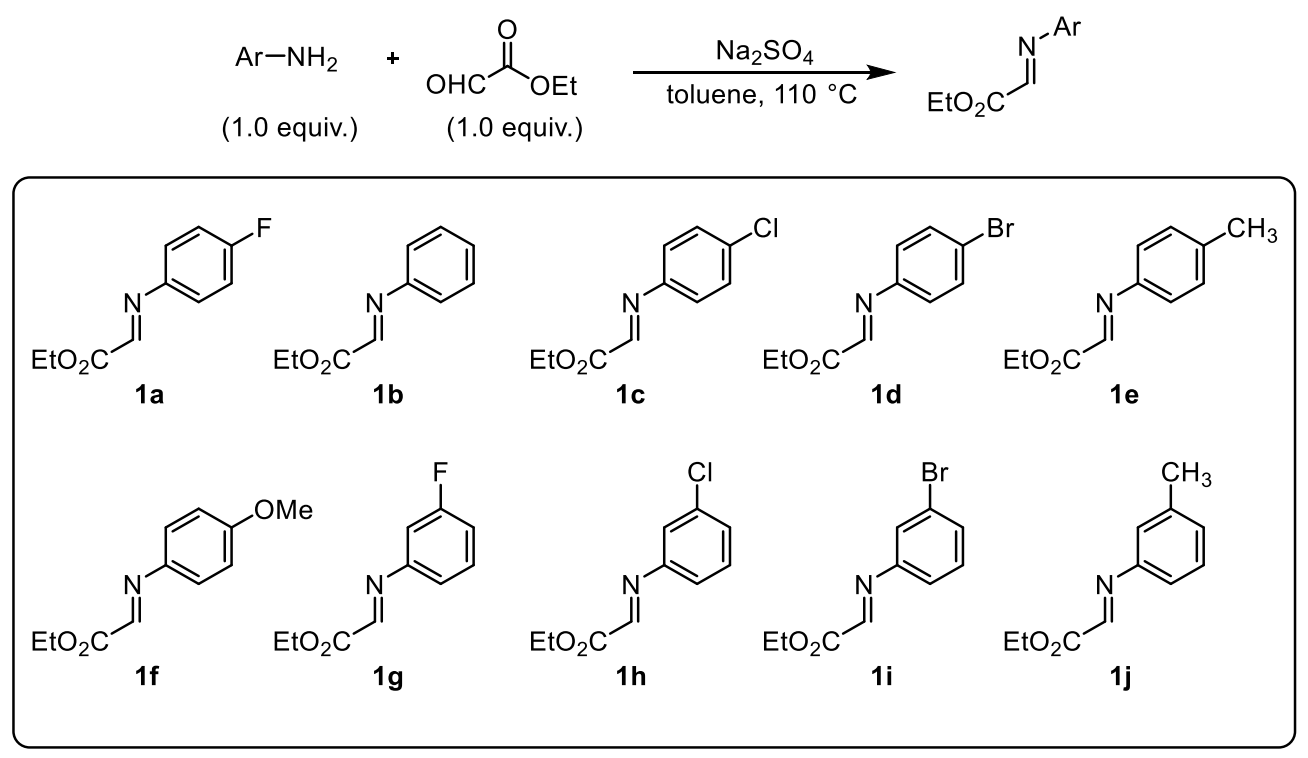

A solution containing ethyl glyoxylate $(1.02 \mathrm{~g}, 10 \mathrm{mmol}, 2.0 \mathrm{~mL}$, ca. $50 \%$ in toluene), a substituted aniline $(10 \mathrm{mmol})$, and anhydrous sodium sulfate $(1.0 \mathrm{~g})$ in toluene $(20 \mathrm{~mL})$ was heated under reflux for $0.5-1.0$ hour to afford the corresponding imine $\mathbf{1}$ in good yields. The solution was filtered and the filtrate was concentrated under reduced pressure. The residue was then purified through a silica gel flash column which was deactivated by triethylamine to afford the pure product $\mathbf{1}$. 


\section{Characterization data for ethyl (arylimino)acetates}

Imine 1a-1f, $\mathbf{1 h}, \mathbf{1} \mathbf{i}$ are known compounds and the characerization data are in agreement with above literatures, while imines $\mathbf{1 g}, \mathbf{1 i}$ are reported for the first time and the characterization data are presented as follows.

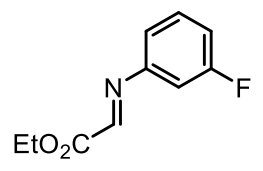

Ethyl-2-((3-fluorophenyl)imino)acetate (1g): yellow oil; ${ }^{1} \mathrm{H}$ NMR (400 MHz, Chloroform- $d$ ) $\delta 7.84$ (s, 1H), $7.38-7.27(\mathrm{~m}, 1 \mathrm{H}), 7.04-6.98(\mathrm{~m}, 2 \mathrm{H}), 6.98-6.91(\mathrm{~m}, 1 \mathrm{H}), 4.38(\mathrm{q}, J=7.2 \mathrm{~Hz}, 2 \mathrm{H}), 1.37(\mathrm{t}, J=$ $7.2 \mathrm{~Hz}, 3 \mathrm{H}) ;{ }^{13} \mathrm{C}\left\{{ }^{1} \mathrm{H}\right\} \mathrm{NMR}(101 \mathrm{MHz}$, Chloroform- $d$ ) $\delta 163.1(\mathrm{~d}, J=247.9 \mathrm{~Hz}), 162.8,152.4,150.6$ (d, $J=8.9 \mathrm{~Hz}), 130.6(\mathrm{~d}, J=8.7 \mathrm{~Hz}), 117.1(\mathrm{~d}, J=3.0 \mathrm{~Hz}), 115.1(\mathrm{~d}, J=21.4 \mathrm{~Hz}), 108.6(\mathrm{~d}, J=23.1 \mathrm{~Hz})$, 62.2, 14.1; ${ }^{19} \mathrm{~F}$ NMR (376 MHz, Chloroform- $d$ ) $\delta$-111.6 - -111.7 (m); IR (neat): 2984, 1744, 1719, 1604, 1587, 1485, 1372, 1300, 1254, $1203 \mathrm{~cm}^{-1}$; HRMS (ESI, m/z): calcd for $\mathrm{C}_{10} \mathrm{H}_{11} \mathrm{FNO}_{2}[\mathrm{M}+\mathrm{H}]^{+} 196.0768$, found 196.0761.<smiles>CCOCCNc1cccc(Br)c1</smiles>

Ethyl-2-((3-bromophenyl)imino)acetate (1i): yellow oil; ${ }^{1} \mathrm{H}$ NMR $(400 \mathrm{MHz}$, Chloroform- $d$ ) $\delta 7.78$ (s, $1 \mathrm{H}), 7.36(\mathrm{~d}, J=7.9 \mathrm{~Hz}, 1 \mathrm{H}), 7.32(\mathrm{~s}, 1 \mathrm{H}), 7.19(\mathrm{t}, J=7.9 \mathrm{~Hz}, 1 \mathrm{H}), 7.12(\mathrm{~d}, J=8.0 \mathrm{~Hz}, 1 \mathrm{H}), 4.33$ (q, $J=$ $7.2 \mathrm{~Hz}, 2 \mathrm{H}), 1.32$ (t, $J=7.2 \mathrm{~Hz}, 3 \mathrm{H}) ;{ }^{13} \mathrm{C}\left\{{ }^{1} \mathrm{H}\right\} \mathrm{NMR}$ (101 MHz, Chloroform- $d$ ) $\delta 162.7,152.5,150.3$, 131.2, 130.6, 124.0, 122.9, 120.3, 62.2, 14.2; IR (neat): 2981, 1743, 1720, 1633, 1567, 1470, 1394, 1301, $1210 \mathrm{~cm}^{-1}$; HRMS (ESI, m/z): calcd for $\mathrm{C}_{10} \mathrm{H}_{11} \mathrm{BrNO}_{2}[\mathrm{M}+\mathrm{H}]^{+} 255.9968$, found 255.9957 .

\section{Condition Optimization of the Three-Component Reaction.}

i. The acid-promoted background [4+2] addition

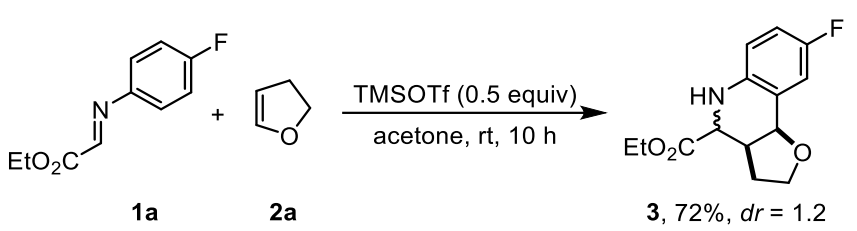

To a flame-dried test tube charged with a stir bar were added ethyl-2-((4-fluorophenyl)imino)acetate 1a (39 $\mathrm{mg}, 0.2 \mathrm{mmol})$ and 2,3-dihydrofuran $(46 \mu \mathrm{L}, 0.6 \mathrm{mmol})$. Acetone $(1 \mathrm{~mL})$ was added through a syringe, followed by the addition of TMSOTf $(18 \mu \mathrm{L}, 0.1 \mathrm{mmol})$. The reaction mixture was stirred at room temperature for $10 \mathrm{~min}$. The progress of the reaction was monitored by TLC. Upon the consumption of imine, the mixture was quenched with $\mathrm{NaHCO}_{3}$ (sat. aq., $2 \mathrm{~mL}$ ), extracted with ether $(3 \mathrm{~mL} \times 3)$ and 
concentrated in vacuo. The residue was then purified through a silica gel flash column to afford the corresponding [4+2] product 3 .

Ethyl 8-fluoro-2,3,3a,4,5,9b-hexahydrofuro[3,2-c]quinoline-4-carboxylate (3): compound 3 was isolated through a silica gel flash column $(\mathrm{PE}:$ EtOAc $=10: 1)$ as colourless oil $(38 \mathrm{mg}, 72 \%$ yield, $d r=$ $1.2)$

Isomer 1 (less polar): ${ }^{1} \mathrm{H}$ NMR $(400 \mathrm{MHz}$, Chloroform- $d) \delta 7.00(\mathrm{dd}, J=9.1,3.0 \mathrm{~Hz}, 1 \mathrm{H}), 6.80(\mathrm{td}, J=$ 8.5, 3.0 Hz, 1H), $6.55(\mathrm{dd}, J=8.8,4.6 \mathrm{~Hz}, 1 \mathrm{H}), 5.15(\mathrm{~d}, J=8.1 \mathrm{~Hz}, 1 \mathrm{H}), 4.36-4.22(\mathrm{~m}, 2 \mathrm{H}), 4.21-4.13$ $(\mathrm{m}, 2 \mathrm{H}), 3.87-3.69(\mathrm{~m}, 2 \mathrm{H}), 3.08(\mathrm{qd}, J=8.1,2.1 \mathrm{~Hz}, 1 \mathrm{H}), 2.04-1.95(\mathrm{~m}, 1 \mathrm{H}), 1.95-1.84(\mathrm{~m}, 1 \mathrm{H})$, $1.33(\mathrm{t}, J=7.1 \mathrm{~Hz}, 3 \mathrm{H}) ;{ }^{13} \mathrm{C}\left\{{ }^{1} \mathrm{H}\right\} \mathrm{NMR}(101 \mathrm{MHz}$, Chloroform- $d$ ) $\delta 171.2,156.6(\mathrm{~d}, J=237.1 \mathrm{~Hz}), 139.6$ (d, $J=2.0 \mathrm{~Hz}), 123.3(\mathrm{~d}, J=6.4 \mathrm{~Hz}), 115.72(\mathrm{~d}, J=22.0 \mathrm{~Hz}), 115.70(\mathrm{~d}, J=7.5 \mathrm{~Hz}), 115.67$ (d, $J=22.9$ $\mathrm{Hz}), 75.4,66.6,61.6,55.4,40.1,25.0,14.2 ;{ }^{19} \mathrm{~F}$ NMR (376 MHz, Chloroform- $d$ ) $\delta$-125.02 - -125.66 (m); IR (neat): 3382, 2975, 2956, 2923, 2873, 2853, 1736, 1502, 1476, 1219, $1165 \mathrm{~cm}^{-1}$; HRMS (ESI, m/z): calcd for $\mathrm{C}_{14} \mathrm{H}_{16} \mathrm{FNO}_{3} \mathrm{Na}[\mathrm{M}+\mathrm{Na}]^{+} 288.1006$, found 288.1012 .

Isomer 2 (more polar): ${ }^{1} \mathrm{H}$ NMR $(400 \mathrm{MHz}$, Chloroform- $d$ ) $\delta 7.05(\mathrm{dd}, J=8.9,2.9 \mathrm{~Hz}, 1 \mathrm{H}), 6.84(\operatorname{td}, J=$ 8.5, 2.9 Hz, 1H), $6.62(\mathrm{dd}, J=8.8,4.6 \mathrm{~Hz}, 1 \mathrm{H}), 4.61(\mathrm{~d}, J=6.3 \mathrm{~Hz}, 1 \mathrm{H}), 4.31$ (br, s, 1H), $4.30-4.21$ (m, $2 \mathrm{H}), 3.96(\mathrm{td}, J=8.5,5.3 \mathrm{~Hz}, 1 \mathrm{H}), 3.82(\mathrm{td}, J=8.5,7.2 \mathrm{~Hz}, 1 \mathrm{H}), 3.59(\mathrm{~d}, J=9.1 \mathrm{~Hz}, 1 \mathrm{H}), 2.65(\mathrm{tdd}, J=$ 8.7, 6.3, $4.7 \mathrm{~Hz}, 1 \mathrm{H}), 2.34-2.25(\mathrm{~m}, 1 \mathrm{H}), 2.25-2.14(\mathrm{~m}, 1 \mathrm{H}), 1.31(\mathrm{t}, J=7.1 \mathrm{~Hz}, 3 \mathrm{H}) ;{ }^{13} \mathrm{C}\left\{{ }^{1} \mathrm{H}\right\} \mathrm{NMR}$ $(101 \mathrm{MHz}$, Chloroform- $d$ ) $\delta$ 172.2, $156.4(\mathrm{~d}, J=237.0 \mathrm{~Hz}), 139.4(\mathrm{~d}, J=1.9 \mathrm{~Hz}), 121.8$ (d, $J=6.9 \mathrm{~Hz})$, $116.30(\mathrm{~d}, J=22.0 \mathrm{~Hz}), 116.10(\mathrm{~d}, J=7.5 \mathrm{~Hz}), 116.00(\mathrm{~d}, J=22.9 \mathrm{~Hz}), 74.7(\mathrm{~d}, J=1.5 \mathrm{~Hz}), 65.8,61.6$, 56.0, 39.1, 29.6, 14.2; ${ }^{19} \mathrm{~F}$ NMR (376 MHz, Chloroform- $d$ ) $\delta$-125.17 - -125.47 (m); IR (neat): 3382, 2924, 1732, 1507, 1472, 1214, $1178 \mathrm{~cm}^{-1}$; HRMS (ESI, m/z): calcd for $\mathrm{C}_{14} \mathrm{H}_{16} \mathrm{FNO}_{3} \mathrm{Na}[\mathrm{M}+\mathrm{Na}]^{+} 288.1006$, found 288.1011 .

\section{ii. General procedure for the optimization of reaction conditions}

To a test tube charged with a stir bar were added imine $1 \mathbf{a}(39 \mathrm{mg}, 0.2 \mathrm{mmol})$ and a photosensitizer $(0.02$ mmol). After the test tube was evacuated and backfilled with $\mathrm{N}_{2}$ three times, a solvent $(1 \mathrm{~mL})$ was added through a syringe, followed by the addition of dihydrofuran 2a (46 $\mu \mathrm{L}, 0.6 \mathrm{mmol})$ and azidotrimethylsilane $(32 \mu \mathrm{L}, 0.24 \mathrm{mmol})$. The reaction mixture was stirred under irradiation ( $3 \mathrm{~W}$ blue LEDs) at room temperature for $24 \mathrm{~h}$. After the removal of solvent under vacuum, 1-fluoro-4-iodobenzene (23 $\mu \mathrm{L}, 0.2 \mathrm{mmol}$ ) was added as an internal standard. The whole mixture was then dissolved in chloroform- $d$ and submitted for quantitative ${ }^{19} \mathrm{~F}$ NMR analysis. 


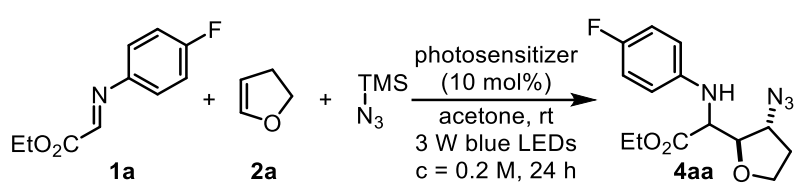

(1 equiv) (3 equiv) (1.2 equiv)

Photosensitizers:

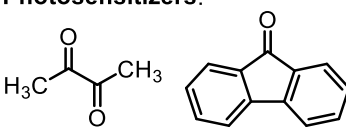

BD

FLN<smiles>O=C(P)c1ccccc1</smiles>

BP

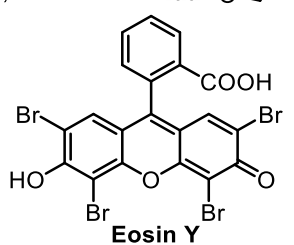

Eosin $Y^{B}$

$\begin{array}{llcc}\text { entry } & \text { catalysts and conditions } & \text { conv. }^{b}(\%) & \text { yield }^{c}(\%) \\ 1 & \text { FLN, standard cond. } & >95 & 89 \\ 2 & \text { BD, standard cond. } & >95 & 70 \\ 3 & \text { BP, standard cond. } & >95 & 65 \\ 4 & \text { Eosin Y, standard cond. } & >95 & 69 \\ 5 & \text { FLN, in } \mathrm{CH}_{3} \mathrm{CN} & >95 & 87 \\ 6 & \text { FLN, in } \mathrm{CH}_{2} \mathrm{Cl} l_{2} & >95 & 78 \\ 7 & \text { FLN, DMF } & >95 & 75 \\ 8 & c=0.1 \text { M } & 93 & 80 \\ 9 & \text { FLN, In darkness } & <5 & <5 \\ 10 & \text { no catalyst, standard cond. } & 90 & 70\end{array}$

${ }^{a}$ Unless otherwise stated, the reaction was performed under $\mathrm{N}_{2}$ with imine $1 \mathrm{a}(0.2 \mathrm{mmol})$, dihydrofuran $(0.6 \mathrm{mmol})$ and $\mathrm{TMSN}_{3}(0.24 \mathrm{mmol})$ in the presence of a photosensitizer $(0.02 \mathrm{mmol})$ in indicating solvent with light irradiation ( $3 \mathrm{~W}$ blue LEDs). ${ }^{b}$ the conversion of 1 a was determined by quantitative ${ }^{19} \mathrm{~F}$ NMR analysis with 4-fluoroiodobenzene as an internal standard. ${ }^{c}$ The yield is also determined by NMR and 4aa was determined to be a pair of diastereomers, $d r \approx 1.2: 1$.<smiles>CCOC(=O)C(Nc1ccc(F)cc1)C1OCCC1N</smiles>

Ethyl 2-(3-azidotetrahydrofuran-2-yl)-2-((4-fluorophenyl)amino)acetate (4aa): compound 4aa was isolated through a silica gel flash column ( $\mathrm{PE}$ : EtOAc $=40: 1)$ as yellow oil $(53 \mathrm{mg}, 86 \%$ yield, $d r=1.2)$.

Isomer 1 (less polar): ${ }^{1} \mathrm{H}$ NMR (600 MHz, Chloroform- $d$ ) $\delta 6.92-6.84(\mathrm{~m}, 2 \mathrm{H}), 6.71-6.64(\mathrm{~m}, 2 \mathrm{H})$, $4.34(\mathrm{~d}, J=8.3 \mathrm{~Hz}, 1 \mathrm{H}), 4.25-4.20(\mathrm{~m}, 2 \mathrm{H}), 4.16(\mathrm{dt}, J=7.5,3.9 \mathrm{~Hz}, 1 \mathrm{H}), 4.12-4.04(\mathrm{~m}, 2 \mathrm{H}), 3.97$ (ddd, $J=8.6,7.7,4.7 \mathrm{~Hz}, 1 \mathrm{H}), 3.89(\mathrm{td}, J=8.4,6.9 \mathrm{~Hz}, 1 \mathrm{H}), 2.22(\mathrm{dq}, J=13.0,7.8 \mathrm{~Hz}, 1 \mathrm{H}), 2.00(\mathrm{ddt}, J$ $=13.0,6.9,4.4 \mathrm{~Hz}, 1 \mathrm{H}), 1.26(\mathrm{t}, J=7.1 \mathrm{~Hz}, 3 \mathrm{H}),{ }^{13} \mathrm{C}\left\{{ }^{1} \mathrm{H}\right\} \mathrm{NMR}(151 \mathrm{MHz}$, Chloroform- $d) \delta 171.3,156.7$ (d, $J=237.0 \mathrm{~Hz}), 143.0(\mathrm{~d}, J=2.2 \mathrm{~Hz}), 115.7(\mathrm{~d}, J=22.5 \mathrm{~Hz}), 115.3(\mathrm{~d}, J=7.6 \mathrm{~Hz}), 84.8,67.7,62.6$, $61.9,60.1,32.3,14.2 ;{ }^{19} \mathrm{~F}$ NMR (376 MHz, Chloroform- $d$ ) $\delta-126.0$ (tt, $J=8.6,4.4 \mathrm{~Hz}$ ); IR (neat): 3361, 2981, 2099, 1731, 1508, 1254, $1214 \mathrm{~cm}^{-1}$; HRMS (ESI, m/z): calcd for $\mathrm{C}_{14} \mathrm{H}_{17} \mathrm{FN}_{4} \mathrm{O}_{3} \mathrm{Na}[\mathrm{M}+\mathrm{Na}]^{+}$ 331.1177 , found 331.1172 .

Isomer 2 (more polar): ${ }^{1} \mathrm{H}$ NMR (600 MHz, Chloroform- $d$ ) $\delta 6.93-6.86(\mathrm{~m}, 2 \mathrm{H}), 6.64-6.58(\mathrm{~m}, 2 \mathrm{H})$, $4.28(\mathrm{~d}, J=10.4 \mathrm{~Hz}, 1 \mathrm{H}), 4.23-4.18(\mathrm{~m}, 2 \mathrm{H}), 4.16(\mathrm{dt}, J=7.8,5.3 \mathrm{~Hz}, 1 \mathrm{H}), 4.13-4.08(\mathrm{~m}, 2 \mathrm{H}), 4.06$ 
(ddd, $J=8.8,7.5,5.0 \mathrm{~Hz}, 1 \mathrm{H}), 3.92(\mathrm{dt}, J=8.8,7.5 \mathrm{~Hz}, 1 \mathrm{H}), 2.29$ (dq, $J=12.9,7.6 \mathrm{~Hz}, 1 \mathrm{H}), 2.06$ (ddt, $J$ $=12.5,7.2,5.1 \mathrm{~Hz}, 1 \mathrm{H}), 1.24(\mathrm{t}, J=7.1 \mathrm{~Hz}, 3 \mathrm{H}) ;{ }^{13} \mathrm{C}\left\{{ }^{1} \mathrm{H}\right\} \mathrm{NMR}(151 \mathrm{MHz}$, Chloroform- $d) \delta 171.4,156.7$ $(\mathrm{d}, J=237.1 \mathrm{~Hz}), 143.3(\mathrm{~d}, J=2.2 \mathrm{~Hz}), 115.9(\mathrm{~d}, J=22.5 \mathrm{~Hz}), 115.3(\mathrm{~d}, J=7.6 \mathrm{~Hz}), 83.8,68.0,62.2$, 61.7, 58.8, 32.3, 14.2; ${ }^{19} \mathrm{~F}$ NMR (376 MHz, Chloroform- $d$ ) $\delta-125.8$ (tt, $J=8.7,3.4 \mathrm{~Hz}$ ); IR (neat): 3372 , 2982, 2100, 1737, 1509, 1258, $1217 \mathrm{~cm}^{-1}$; HRMS (ESI, m/z): calcd for $\mathrm{C}_{14} \mathrm{H}_{17} \mathrm{FN}_{4} \mathrm{O}_{3} \mathrm{Na}[\mathrm{M}+\mathrm{Na}]^{+}$ 331.1177 , found 331.1164 .

\section{The Preparation of $\gamma$-Azido Amino Esters}

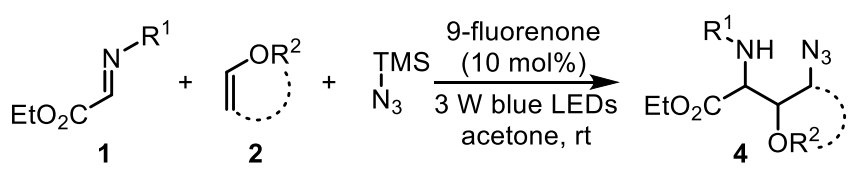

The general procedure for the visible light enabled radical addition cascade: To a test tube charged with a stir bar were added an imine $1(0.2 \mathrm{mmol})$ and 9-fluorenone $(3.6 \mathrm{mg}, 0.02 \mathrm{mmol})$. After the test tube was evacuated and backfilled with $\mathrm{N}_{2}$ three times, acetone $(1 \mathrm{~mL})$ was added through a syringe, followed by the addition of an enol ether $2(0.6 \mathrm{mmol})$ and azidotrimethylsilane ( $32 \mu \mathrm{L}, 0.24 \mathrm{mmol})$. The reaction mixture was stirred under irradiation ( $3 \mathrm{~W}$ blue LEDs) at room temperature for $24 \mathrm{~h}$. The progress of the reaction was monitored by TLC. Upon the consumption of imine, the mixture was concentrated in vacuo. The residue was then purified through a silica gel flash column to afford the corresponding product 4.

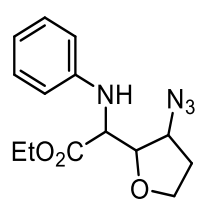

Ethyl 2-(3-azidotetrahydrofuran-2-yl)-2-(phenylamino)acetate (4ba): compound 4ba was isolated through a silica gel flash column $(\mathrm{PE}:$ EtOAc $=40: 1)$ as yellow oil $(47 \mathrm{mg}, 81 \%$ yield, $d r=1.2)$.

Isomer 1 (less polar): ${ }^{1} \mathrm{H}$ NMR (600 MHz, Chloroform- $d$ ) $\delta 7.23-7.16(\mathrm{~m}, 2 \mathrm{H}), 6.82-6.76(\mathrm{~m}, 1 \mathrm{H})$, $6.77-6.71(\mathrm{~m}, 2 \mathrm{H}), 4.43(\mathrm{~d}, J=8.5 \mathrm{~Hz}, 1 \mathrm{H}), 4.24(\mathrm{q}, J=7.1 \mathrm{~Hz}, 2 \mathrm{H}), 4.21-4.15(\mathrm{~m}, 2 \mathrm{H}), 4.11(\mathrm{dd}, J=$ 5.2, 4.2 Hz, 1H), 3.99 (ddd, $J=8.7,7.8,4.6 \mathrm{~Hz}, 1 \mathrm{H}), 3.90(\mathrm{td}, J=8.4,6.9 \mathrm{~Hz}, 1 \mathrm{H}), 2.24$ (dq, $J=13.1$, $7.8 \mathrm{~Hz}, 1 \mathrm{H}), 2.01$ (ddt, $J=13.1,6.9,4.3 \mathrm{~Hz}, 1 \mathrm{H}), 1.27(\mathrm{t}, J=7.1 \mathrm{~Hz}, 3 \mathrm{H}) ;{ }^{13} \mathrm{C}\left\{{ }^{1} \mathrm{H}\right\} \mathrm{NMR}(151 \mathrm{MHz}$, Chloroform- $d$ ) $\delta 171.3,146.7,129.3,119.1,114.2,84.9,67.7,62.7,61.8,59.4,32.3,14.2$; IR (neat): 3360, 2925, 2098, 1731, 1602, 1505, 1255, $1181 \mathrm{~cm}^{-1}$; HRMS (ESI, m/z): calcd for $\mathrm{C}_{14} \mathrm{H}_{18} \mathrm{~N}_{4} \mathrm{O}_{3} \mathrm{Na}[\mathrm{M}+\mathrm{Na}]^{+}$ 313.1271, found 313.1279.

Isomer 2 (more polar): ${ }^{1} \mathrm{H}$ NMR (600 MHz, Chloroform- $d$ ) $\delta 7.23-7.17(\mathrm{~m}, 2 \mathrm{H}), 6.82-6.76(\mathrm{~m}, 1 \mathrm{H})$, $6.71-6.64(\mathrm{~m}, 2 \mathrm{H}), 4.39(\mathrm{~d}, J=10.2 \mathrm{~Hz}, 1 \mathrm{H}), 4.22(\mathrm{dd}, J=10.1,2.4 \mathrm{~Hz}, 1 \mathrm{H}), 4.21$ (q, $J=7.1 \mathrm{~Hz}, 2 \mathrm{H})$, $4.16(\mathrm{dt}, J=7.7,5.3 \mathrm{~Hz}, 1 \mathrm{H}), 4.12(\mathrm{dd}, J=5.7,2.5 \mathrm{~Hz}, 1 \mathrm{H}), 4.07(\mathrm{ddd}, J=8.8,7.5,5.1 \mathrm{~Hz}, 1 \mathrm{H}), 3.92(\mathrm{dt}$, 
$J=8.8,7.5 \mathrm{~Hz}, 1 \mathrm{H}), 2.28(\mathrm{dq}, J=12.9,7.6 \mathrm{~Hz}, 1 \mathrm{H}), 2.06(\mathrm{ddt}, J=12.5,7.2,5.1 \mathrm{~Hz}, 1 \mathrm{H}), 1.26(\mathrm{t}, J=7.1$ $\mathrm{Hz}, 3 \mathrm{H}) ;{ }^{13} \mathrm{C}\left\{{ }^{1} \mathrm{H}\right\} \mathrm{NMR}(151 \mathrm{MHz}$, Chloroform- $d) \delta$ 171.4, 147.0, 129.5, 119.1, 114.0, 83.9, 68.0, 62.2, 61.7, 57.9, 32.3, 14.2; IR (neat): 3374, 2925, 2102, 1740, 1648, 1602, 1502, 1257, $1185 \mathrm{~cm}^{-1}$; HRMS (ESI, m/z): calcd for $\mathrm{C}_{14} \mathrm{H}_{18} \mathrm{~N}_{4} \mathrm{O}_{3} \mathrm{Na}[\mathrm{M}+\mathrm{Na}]^{+}$313.1271, found 313.1281.

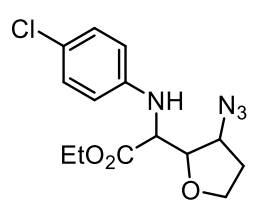

Ethyl 2-(3-azidotetrahydrofuran-2-yl)-2-((4-chlorophenyl)amino)acetate (4ca): compound 4ca was isolated through a silica gel flash column (PE: EtOAc $=40: 1)$ as yellow oil (56 mg, 86\% yield, $d r=1.3)$. Isomer 1 (less polar): ${ }^{1} \mathrm{H}$ NMR (400 MHz, Chloroform- $d$ ) $\delta 7.17-7.08(\mathrm{~m}, 2 \mathrm{H}), 6.70-6.62(\mathrm{~m}, 2 \mathrm{H})$, $4.47(\mathrm{~d}, J=8.1 \mathrm{~Hz}, 1 \mathrm{H}), 4.24(\mathrm{q}, J=7.2 \mathrm{~Hz}, 2 \mathrm{H}), 4.18-4.11(\mathrm{~m}, 2 \mathrm{H}), 4.08(\mathrm{t}, J=4.5 \mathrm{~Hz}, 1 \mathrm{H}), 3.96(\mathrm{ddd}$, $J=8.7,7.7,4.7 \mathrm{~Hz}, 1 \mathrm{H}), 3.88(\mathrm{td}, J=8.3,6.9 \mathrm{~Hz}, 1 \mathrm{H}), 2.22(\mathrm{dq}, J=13.1,7.7 \mathrm{~Hz}, 1 \mathrm{H}), 2.00(\mathrm{ddt}, J=$ 13.0, 6.9, 4.5 Hz, 1H), 1.27 (t, $J=7.2 \mathrm{~Hz}, 3 \mathrm{H}) ;{ }^{13} \mathrm{C}\left\{{ }^{1} \mathrm{H}\right\} \mathrm{NMR}(101 \mathrm{MHz}$, Chloroform- $d$ ) $\delta 171.0,145.3$, 129.1, 123.7, 115.3, 84.8, 67.7, 62.5, 62.0, 59.4, 32.3, 14.2; IR (neat): 3368, 2981, 2098, 1730, 1599, 1493, 1252, $1178 \mathrm{~cm}^{-1}$; HRMS (ESI, m/z): calcd for $\mathrm{C}_{14} \mathrm{H}_{17} \mathrm{ClN}_{4} \mathrm{O}_{3} \mathrm{Na}[\mathrm{M}+\mathrm{Na}]^{+} 347.0881$, found 347.0862 .

Isomer 2 (more polar): ${ }^{1} \mathrm{H}$ NMR (400 MHz, Chloroform- $d$ ) $\delta 7.18-7.09(\mathrm{~m}, 2 \mathrm{H}), 6.64-6.54(\mathrm{~m}, 2 \mathrm{H})$, $4.40(\mathrm{~d}, J=10.3 \mathrm{~Hz}, 1 \mathrm{H}), 4.21(\mathrm{q}, J=7.1 \mathrm{~Hz}, 2 \mathrm{H}), 4.19-4.01(\mathrm{~m}, 4 \mathrm{H}), 3.92(\mathrm{dt}, J=8.8,7.4 \mathrm{~Hz}, 1 \mathrm{H})$, $2.28(\mathrm{dq}, J=13.1,7.5 \mathrm{~Hz}, 1 \mathrm{H}), 2.06(\mathrm{ddt}, J=12.5,7.3,5.0 \mathrm{~Hz}, 1 \mathrm{H}), 1.25(\mathrm{t}, J=7.1 \mathrm{~Hz}, 3 \mathrm{H}) ;{ }^{13} \mathrm{C}\left\{{ }^{1} \mathrm{H}\right\} \mathrm{NMR}$ $(101 \mathrm{MHz}$, Chloroform- $d$ ) $\delta 171.2,145.6,129.3,123.8,115.2,83.7,68.0,62.1,61.8,57.9,32.2,14.2$; IR (neat): 3370, 2981, 2101, 1730, 1598, 1493, 1251, $1179 \mathrm{~cm}^{-1} ;$ HRMS (ESI, m/z): calcd for $\mathrm{C}_{14} \mathrm{H}_{17} \mathrm{ClN}_{4} \mathrm{O}_{3} \mathrm{Na}[\mathrm{M}+\mathrm{Na}]^{+}$347.0881, found 347.0859.

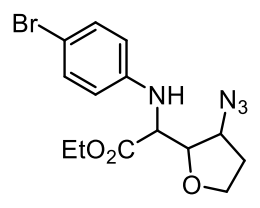

Ethyl 2-(3-azidotetrahydrofuran-2-yl)-2-((4-bromophenyl)amino)acetate (4da): compound 4da was isolated through a silica gel flash column $(\mathrm{PE}:$ EtOAc $=30: 1)$ as yellow oil $(63 \mathrm{mg}, 86 \%$ yield, $d r=1.3)$. Isomer 1 (less polar): ${ }^{1} \mathrm{H}$ NMR (400 MHz, Chloroform- $d$ ) $\delta 7.30-7.20(\mathrm{~m}, 2 \mathrm{H}), 6.66-6.57(\mathrm{~m}, 2 \mathrm{H})$, $4.49(\mathrm{~d}, J=8.0 \mathrm{~Hz}, 1 \mathrm{H}), 4.23(\mathrm{q}, J=7.1 \mathrm{~Hz}, 2 \mathrm{H}), 4.19-4.10(\mathrm{~m}, 2 \mathrm{H}), 4.08(\mathrm{t}, J=4.5 \mathrm{~Hz}, 1 \mathrm{H}), 3.96(\mathrm{ddd}$, $J=8.7,7.7,4.7 \mathrm{~Hz}, 1 \mathrm{H}), 3.88(\mathrm{td}, J=8.3,6.9 \mathrm{~Hz}, 1 \mathrm{H}), 2.21(\mathrm{dq}, J=13.1,7.7 \mathrm{~Hz}, 1 \mathrm{H}), 2.00(\mathrm{ddt}, J=$ 13.1, 6.9, 4.5 Hz, 1H), 1.27 (t, $J=7.1 \mathrm{~Hz}, 3 \mathrm{H}) ;{ }^{13} \mathrm{C}\left\{{ }^{1} \mathrm{H}\right\} \mathrm{NMR}(101 \mathrm{MHz}$, Chloroform- $d$ ) $\delta 170.9,145.7$, 132.0, 115.8, 110.8, 84.7, 67.7, 62.4, 62.0, 59.3, 32.3, 14.2; IR (neat): 3363, 2980, 2100, 1730, 1593, 
1489, 1255, $1180 \mathrm{~cm}^{-1}$; HRMS (ESI, m/z): calcd for $\mathrm{C}_{14} \mathrm{H}_{17} \mathrm{BrN}_{4} \mathrm{O}_{3} \mathrm{Na}[\mathrm{M}+\mathrm{Na}]^{+}$391.0376, found 391.0361 .

Isomer 2 (more polar): ${ }^{1} \mathrm{H}$ NMR (400 MHz, Chloroform- $d$ ) $\delta 7.29-7.22(\mathrm{~m}, 2 \mathrm{H}), 6.56-6.49(\mathrm{~m}, 2 \mathrm{H})$, $4.39(\mathrm{~d}, J=10.2 \mathrm{~Hz}, 1 \mathrm{H}), 4.19(\mathrm{q}, J=7.1 \mathrm{~Hz}, 2 \mathrm{H}), 4.13(\mathrm{dd}, J=10.2,2.3 \mathrm{~Hz}, 1 \mathrm{H}), 4.13-3.99(\mathrm{~m}, 3 \mathrm{H})$, $3.90(\mathrm{dt}, J=8.8,7.5 \mathrm{~Hz}, 1 \mathrm{H}), 2.26(\mathrm{dq}, J=13.0,7.4 \mathrm{~Hz}, 1 \mathrm{H}), 2.04(\mathrm{ddt}, J=12.5,7.3,5.0 \mathrm{~Hz}, 1 \mathrm{H}), 1.23$ $(\mathrm{t}, J=7.1 \mathrm{~Hz}, 3 \mathrm{H}) ;{ }^{13} \mathrm{C}\left\{{ }^{1} \mathrm{H}\right\} \mathrm{NMR}(101 \mathrm{MHz}$, Chloroform- $d) \delta 171.1,146.1,132.2,115.6,110.9,83.6$, 68.0, 62.1, 61.9, 57.8, 32.2, 14.2; IR (neat): 3374, 2979, 2099, 1732, 1593, 1496, 1251, $1181 \mathrm{~cm}^{-1}$; HRMS (ESI, m/z): calcd for $\mathrm{C}_{14} \mathrm{H}_{17} \mathrm{BrN}_{4} \mathrm{O}_{3} \mathrm{Na}[\mathrm{M}+\mathrm{Na}]^{+}$391.0376, found 391.0355.

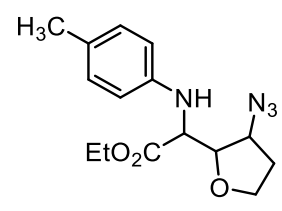

Ethyl 2-(3-azidotetrahydrofuran-2-yl)-2-(p-tolylamino)acetate (4ea): compound 4ea was isolated through a silica gel flash column $(\mathrm{PE}:$ EtOAc $=30: 1)$ as yellow oil $(51 \mathrm{mg}, 84 \%$ yield, $d r=1.1)$.

Isomer 1 (less polar): ${ }^{1} \mathrm{H}$ NMR (600 MHz, Chloroform- $d$ ) $\delta 7.00(\mathrm{~d}, J=8.1 \mathrm{~Hz}, 2 \mathrm{H}), 6.70-6.61(\mathrm{~m}, 2 \mathrm{H})$, $4.29(\mathrm{~d}, J=8.3 \mathrm{~Hz}, 1 \mathrm{H}), 4.23(\mathrm{q}, J=7.2 \mathrm{~Hz}, 2 \mathrm{H}), 4.18(\mathrm{dt}, J=7.6,3.9 \mathrm{~Hz}, 1 \mathrm{H}), 4.14-4.08(\mathrm{~m}, 2 \mathrm{H}), 4.00$ (ddd, $J=8.6,7.7,4.5 \mathrm{~Hz}, 1 \mathrm{H}), 3.90(\mathrm{td}, J=8.5,6.9 \mathrm{~Hz}, 1 \mathrm{H}), 2.28-2.20(\mathrm{~m}, 4 \mathrm{H}), 2.00$ (ddt, $J=13.0,6.8$, $4.2 \mathrm{~Hz}, 1 \mathrm{H}), 1.27$ (t, $J=7.2 \mathrm{~Hz}, 3 \mathrm{H}) ;{ }^{13} \mathrm{C}\left\{{ }^{1} \mathrm{H}\right\} \mathrm{NMR}(151 \mathrm{MHz}$, Chloroform-d) $\delta 171.5,144.3,129.8$, 128.4, 114.4, 85.0, 67.7, 62.8, 61.7, 59.8, 32.3, 20.4, 14.2; IR (neat): 3363, 2981, 2099, 1731, 1616, 1518, 1257, $1183 \mathrm{~cm}^{-1}$; HRMS (ESI, m/z): calcd for $\mathrm{C}_{15} \mathrm{H}_{20} \mathrm{~N}_{4} \mathrm{O}_{3} \mathrm{Na}[\mathrm{M}+\mathrm{Na}]^{+} 327.1428$, found 327.1433 .

Isomer 2 (more polar): ${ }^{1} \mathrm{H}$ NMR (400 MHz, Chloroform- $d$ ) $\delta 7.06-6.97(\mathrm{~m}, 2 \mathrm{H}), 6.67-6.55(\mathrm{~m}, 2 \mathrm{H})$, $4.28(\mathrm{~d}, J=10.4 \mathrm{~Hz}, 1 \mathrm{H}), 4.21(\mathrm{q}, J=7.1 \mathrm{~Hz}, 2 \mathrm{H}), 4.22-4.13(\mathrm{~m}, 2 \mathrm{H}), 4.11(\mathrm{dd}, J=5.6,2.5 \mathrm{~Hz}, 1 \mathrm{H})$, 4.07 (ddd, $J=8.8,7.5,5.0 \mathrm{~Hz}, 1 \mathrm{H}), 3.92(\mathrm{dt}, J=8.7,7.4 \mathrm{~Hz}, 1 \mathrm{H}), 2.33-2.23(\mathrm{~m}, 1 \mathrm{H}), 2.25(\mathrm{~s}, 3 \mathrm{H}), 2.05$ (ddt, $J=12.4,7.2,5.0 \mathrm{~Hz}, 1 \mathrm{H}), 1.26(\mathrm{t}, J=7.1 \mathrm{~Hz}, 3 \mathrm{H}) ;{ }^{13} \mathrm{C}\left\{{ }^{1} \mathrm{H}\right\} \mathrm{NMR}(101 \mathrm{MHz}$, Chloroform-d) $\delta 171.6$, 144.7, 129.9, 128.4, 114.2, 84.0, 68.0, 62.3, 61.6, 58.3, 32.3, 20.4, 14.2; IR (neat): 3375, 2980, 2100, 1739, 1616, 1518, 1252, $1183 \mathrm{~cm}^{-1}$; HRMS (ESI, m/z): calcd for $\mathrm{C}_{15} \mathrm{H}_{20} \mathrm{~N}_{4} \mathrm{O}_{3} \mathrm{Na}[\mathrm{M}+\mathrm{Na}]^{+} 327.1428$, found 327.1437.

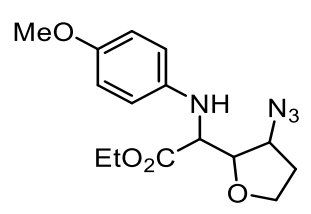

Ethyl 2-(3-azidotetrahydrofuran-2-yl)-2-((4-methoxyphenyl)amino)acetate (4fa): compound 4 fa was isolated through a silica gel flash column (PE: EtOAc $=20: 1)$ as yellow oil (40 $\mathrm{mg}, 63 \%$ yield, $d r=1.3$ ). Isomer 1 (less polar): ${ }^{1} \mathrm{H}$ NMR (400 MHz, Chloroform- $d$ ) $\delta 6.80-6.74(\mathrm{~m}, 2 \mathrm{H}), 6.73-6.67(\mathrm{~m}, 2 \mathrm{H})$, $4.21(\mathrm{q}, J=7.1 \mathrm{~Hz}, 2 \mathrm{H}), 4.20-4.15(\mathrm{~m}, 2 \mathrm{H}), 4.10-4.03(\mathrm{~m}, 2 \mathrm{H}), 3.99$ (ddd, $J=8.7,7.8,4.5 \mathrm{~Hz}, 1 \mathrm{H})$, 
$3.89(\mathrm{td}, J=8.4,6.9 \mathrm{~Hz}, 1 \mathrm{H}), 3.74(\mathrm{~s}, 3 \mathrm{H}), 2.23(\mathrm{dq}, J=13.2,7.8 \mathrm{~Hz}, 1 \mathrm{H}), 1.99$ (dddd, $J=13.1,6.9,4.5$, $3.8 \mathrm{~Hz}, 1 \mathrm{H}), 1.25(\mathrm{t}, J=7.1 \mathrm{~Hz}, 3 \mathrm{H}) ;{ }^{13} \mathrm{C}\left\{{ }^{1} \mathrm{H}\right\} \mathrm{NMR}(101 \mathrm{MHz}$, Chloroform- $d$ ) $\delta 171.6,153.2,140.6$, 115.8, 114.8, 84.9, 67.7, 62.8, 61.7, 60.6, 55.7, 32.2, 14.2; IR (neat): 3354, 2981, 2097, 1730, 1511, 1237 , $1181 \mathrm{~cm}^{-1}$; HRMS (ESI, m/z): calcd for $\mathrm{C}_{15} \mathrm{H}_{20} \mathrm{~N}_{4} \mathrm{O}_{4} \mathrm{Na}[\mathrm{M}+\mathrm{Na}]^{+} 343.1377$, found 343.1379.

Isomer 2 (more polar): ${ }^{1} \mathrm{H}$ NMR (400 MHz, Chloroform- $d$ ) $\delta 6.82-6.75(\mathrm{~m}, 2 \mathrm{H}), 6.69-6.61(\mathrm{~m}, 2 \mathrm{H})$, $4.24-4.17(\mathrm{~m}, 3 \mathrm{H}), 4.16-4.02(\mathrm{~m}, 4 \mathrm{H}), 3.91(\mathrm{dt}, J=8.8,7.5 \mathrm{~Hz}, 1 \mathrm{H}), 3.74(\mathrm{~s}, 3 \mathrm{H}), 2.29(\mathrm{dq}, J=12.9$, $7.7 \mathrm{~Hz}, 1 \mathrm{H}), 2.05$ (ddt, $J=12.4,7.2,5.0 \mathrm{~Hz}, 1 \mathrm{H}), 1.24(\mathrm{t}, J=7.1 \mathrm{~Hz}, 3 \mathrm{H}) ;{ }^{13} \mathrm{C}\left\{{ }^{1} \mathrm{H}\right\} \mathrm{NMR}(101 \mathrm{MHz}$, Chloroform- $d$ ) $\delta 171.7,153.2,141.0,115.8,114.9,84.0,68.0,62.3,61.6,59.2,55.7,32.3,14.2$; IR (neat): 3371, 2916, 2100, 1739, 1511, 1234, $1181 \mathrm{~cm}^{-1}$; HRMS (ESI, m/z): calcd for $\mathrm{C}_{15} \mathrm{H}_{20} \mathrm{~N}_{4} \mathrm{O}_{4} \mathrm{Na}[\mathrm{M}+\mathrm{Na}]^{+}$ 343.1377 , found 343.1368 .

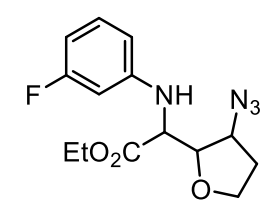

Ethyl 2-(3-azidotetrahydrofuran-2-yl)-2-((3-fluorophenyl)amino)acetate (4ga): compound 4ga was isolated through a silica gel flash column $(\mathrm{PE}$ : EtOAc $=20: 1)$ as colourless oil $(52 \mathrm{mg}, 84 \%$ yield, $d r=$ 1.2).

Isomer 1 (less polar): ${ }^{1} \mathrm{H}$ NMR (400 MHz, Chloroform- $d$ ) $\delta 7.17-7.04(\mathrm{~m}, 1 \mathrm{H}), 6.54-6.42(\mathrm{~m}, 3 \mathrm{H})$, $4.58(\mathrm{~d}, J=8.1 \mathrm{~Hz}, 1 \mathrm{H}), 4.25(\mathrm{q}, J=7.2 \mathrm{~Hz}, 2 \mathrm{H}), 4.20-4.13(\mathrm{~m}, 2 \mathrm{H}), 4.13-4.05(\mathrm{~m}, 1 \mathrm{H}), 3.97(\mathrm{td}, J=$ 8.2, $4.7 \mathrm{~Hz}, 1 \mathrm{H}), 3.94-3.83(\mathrm{~m}, 1 \mathrm{H}), 2.29-2.17(\mathrm{~m}, 1 \mathrm{H}), 2.01(\mathrm{ddt}, J=11.8,7.5,4.5 \mathrm{~Hz}, 1 \mathrm{H}), 1.28(\mathrm{t}$, $J=7.2 \mathrm{~Hz}, 3 \mathrm{H}) ;{ }^{13} \mathrm{C}\left\{{ }^{1} \mathrm{H}\right\} \mathrm{NMR}(101 \mathrm{MHz}$, Chloroform- $d) \delta 170.9,163.9(\mathrm{~d}, J=243.7 \mathrm{~Hz}), 148.5(\mathrm{~d}, J=$ $10.6 \mathrm{~Hz}), 130.4$ (d, $J=10.0 \mathrm{~Hz}), 109.9$ (d, $J=2.5 \mathrm{~Hz}), 105.5(\mathrm{~d}, J=21.4 \mathrm{~Hz}), 101.1$ (d, $J=25.5 \mathrm{~Hz})$, 84.7, 67.7, 62.4, 62.0, 59.2, 32.3, 14.2; ${ }^{19} \mathrm{~F}$ NMR (376 MHz, Chloroform- $d$ ) $\delta-110.50--113.60$ (m); IR (neat): 3369, 2982, 2099, 1730, 1615, 1592, 1513, 1256, $1178 \mathrm{~cm}^{-1}$; HRMS (ESI, m/z): calcd for $\mathrm{C}_{14} \mathrm{H}_{17} \mathrm{FN}_{4} \mathrm{O}_{3} \mathrm{Na}[\mathrm{M}+\mathrm{Na}]^{+} 331.1177$, found 331.1165 .

Isomer 2 (more polar): ${ }^{1} \mathrm{H}$ NMR (400 MHz, Chloroform- $d$ ) $\delta 7.12(\mathrm{td}, J=8.2,6.6 \mathrm{~Hz}, 1 \mathrm{H}), 6.53-6.39$ $(\mathrm{m}, 2 \mathrm{H}), 6.36(\mathrm{dt}, J=11.3,2.3 \mathrm{~Hz}, 1 \mathrm{H}), 4.51(\mathrm{~d}, J=10.1 \mathrm{~Hz}, 1 \mathrm{H}), 4.22(\mathrm{q}, J=7.1 \mathrm{~Hz}, 2 \mathrm{H}), 4.17(\mathrm{dd}, J=$ 10.2, $2.2 \mathrm{~Hz}, 1 \mathrm{H}), 4.16-4.09(\mathrm{~m}, 2 \mathrm{H}), 4.06(\mathrm{ddd}, J=8.8,6.8,4.3 \mathrm{~Hz}, 1 \mathrm{H}), 3.92(\mathrm{dt}, J=8.8,7.5 \mathrm{~Hz}, 1 \mathrm{H})$, $2.36-2.23(\mathrm{~m}, 1 \mathrm{H}), 2.12-2.02(\mathrm{~m}, 1 \mathrm{H}), 1.26(\mathrm{t}, J=7.1 \mathrm{~Hz}, 3 \mathrm{H}) ;{ }^{13} \mathrm{C}\left\{{ }^{1} \mathrm{H}\right\} \mathrm{NMR}(101 \mathrm{MHz}$, Chloroformd) $\delta 171.0,164.0(\mathrm{~d}, J=243.9 \mathrm{~Hz}), 148.8(\mathrm{~d}, J=10.3 \mathrm{~Hz}), 130.6(\mathrm{~d}, J=10.2 \mathrm{~Hz}), 109.6(\mathrm{~d}, J=2.6 \mathrm{~Hz})$, $105.6(\mathrm{~d}, J=21.4 \mathrm{~Hz}), 100.9(\mathrm{~d}, J=25.3 \mathrm{~Hz}), 83.6,67.9,62.1,61.8,57.6,32.2,14.1 ;{ }^{19} \mathrm{~F} \mathrm{NMR}(376 \mathrm{MHz}$, Chloroform- $d$ ) $\delta-111.45$ - -113.04 (m); IR (neat): 3384, 2982, 2102, 1738, 1615, 1591, 1511, 1255, 1177 $\mathrm{cm}^{-1}$; HRMS (ESI, m/z): calcd for $\mathrm{C}_{14} \mathrm{H}_{17} \mathrm{FN}_{4} \mathrm{O}_{3} \mathrm{Na}[\mathrm{M}+\mathrm{Na}]^{+} 331.1177$, found 331.1167. 


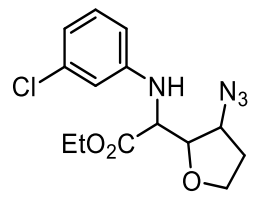

Ethyl 2-(3-azidotetrahydrofuran-2-yl)-2-((3-chlorophenyl)amino)acetate (4ha): compound 4ha was isolated through a silica gel flash column (PE: EtOAc $=30: 1)$ as yellow oil (51 mg, 79\% yield, $d r=1.1)$. Isomer 1 (less polar): ${ }^{1} \mathrm{H}$ NMR (400 MHz, Chloroform- $d$ ) $\delta 7.08(\mathrm{t}, J=8.0 \mathrm{~Hz}, 1 \mathrm{H}), 6.80-6.73(\mathrm{~m}, 2 \mathrm{H})$, $6.64-6.55(\mathrm{~m}, 1 \mathrm{H}), 4.54(\mathrm{~d}, J=8.1 \mathrm{~Hz}, 1 \mathrm{H}), 4.25(\mathrm{q}, J=7.1 \mathrm{~Hz}, 2 \mathrm{H}), 4.19-4.12(\mathrm{~m}, 2 \mathrm{H}), 4.12-4.07$ $(\mathrm{m}, 1 \mathrm{H}), 3.97(\mathrm{td}, J=8.2,4.7 \mathrm{~Hz}, 1 \mathrm{H}), 3.93-3.85(\mathrm{~m}, 1 \mathrm{H}), 2.31-2.16(\mathrm{~m}, 1 \mathrm{H}), 2.01$ (ddt, $J=11.9,7.5$, $4.5 \mathrm{~Hz}, 1 \mathrm{H}), 1.28(\mathrm{t}, J=7.1 \mathrm{~Hz}, 3 \mathrm{H}) ;{ }^{13} \mathrm{C}\left\{{ }^{1} \mathrm{H}\right\} \mathrm{NMR}$ (101 MHz, Chloroform- $d$ ) $\delta 170.8,147.9,135.0$, 130.3, 119.0, 114.0, 112.4, 84.7, 67.7, 62.4, 62.0, 59.1, 32.3, 14.2; IR (neat): 3368, 2981, 2098, 1730, 1596, 1505, 1255, $1189 \mathrm{~cm}^{-1}$; HRMS (ESI, m/z): calcd for $\mathrm{C}_{14} \mathrm{H}_{17} \mathrm{ClN}_{4} \mathrm{O}_{3} \mathrm{Na}[\mathrm{M}+\mathrm{Na}]^{+} 347.0881$, found 347.0875 .

Isomer 2 (more polar): ${ }^{1} \mathrm{H}$ NMR $(400 \mathrm{MHz}$, Chloroform- $d$ ) $\delta 7.10(\mathrm{t}, J=8.0 \mathrm{~Hz}, 1 \mathrm{H}), 6.75$ (ddd, $J=7.9$, $1.9,0.9 \mathrm{~Hz}, 1 \mathrm{H}), 6.65(\mathrm{t}, J=2.1 \mathrm{~Hz}, 1 \mathrm{H}), 6.53(\mathrm{ddd}, J=8.2,2.4,0.9 \mathrm{~Hz}, 1 \mathrm{H}), 4.47(\mathrm{~d}, J=10.1 \mathrm{~Hz}, 1 \mathrm{H})$, $4.22(\mathrm{q}, J=7.2 \mathrm{~Hz}, 2 \mathrm{H}), 4.17(\mathrm{dd}, J=10.1,2.0 \mathrm{~Hz}, 1 \mathrm{H}), 4.13-4.08(\mathrm{~m}, 2 \mathrm{H}), 4.08-4.02(\mathrm{~m}, 1 \mathrm{H}), 3.92$ $(\mathrm{dt}, J=8.8,7.4 \mathrm{~Hz}, 1 \mathrm{H}), 2.33-2.22(\mathrm{~m}, 1 \mathrm{H}), 2.12-2.01(\mathrm{~m}, 1 \mathrm{H}), 1.26(\mathrm{t}, J=7.1 \mathrm{~Hz}, 3 \mathrm{H}) ;{ }^{13} \mathrm{C}\left\{{ }^{1} \mathrm{H}\right\} \mathrm{NMR}$ (101 MHz, Chloroform- $d$ ) $\delta 171.0,148.2,135.2,130.4,119.0,113.9,112.1,83.6,67.9,62.1,61.9,57.5$, 32.2, 14.2; IR (neat): 3378, 2981, 2103, 1738, 1597, 1503, 1259, $1195 \mathrm{~cm}^{-1}$; HRMS (ESI, m/z): calcd for $\mathrm{C}_{14} \mathrm{H}_{17} \mathrm{ClN}_{4} \mathrm{O}_{3} \mathrm{Na}[\mathrm{M}+\mathrm{Na}]^{+} 347.0881$, found 347.0872 .

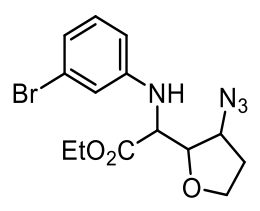

Ethyl 2-(3-azidotetrahydrofuran-2-yl)-2-((3-bromophenyl)amino)acetate (4ia): compound 4ia was isolated through a silica gel flash column $(\mathrm{PE}:$ EtOAc $=30: 1)$ as yellow oil $(60 \mathrm{mg}, 81 \%$ yield, $d r=1.1)$. Isomer 1 (less polar): ${ }^{1} \mathrm{H}$ NMR (400 MHz, Chloroform- $d$ ) $\delta 7.07-6.98(\mathrm{~m}, 1 \mathrm{H}), 6.93-6.85(\mathrm{~m}, 2 \mathrm{H})$, $6.65(\mathrm{ddd}, J=8.2,2.3,1.0 \mathrm{~Hz}, 1 \mathrm{H}), 4.53(\mathrm{~d}, J=8.0 \mathrm{~Hz}, 1 \mathrm{H}), 4.25(\mathrm{q}, J=7.1 \mathrm{~Hz}, 2 \mathrm{H}), 4.19-4.11(\mathrm{~m}$, $2 \mathrm{H}), 4.11-4.07(\mathrm{~m}, 1 \mathrm{H}), 3.97(\mathrm{ddd}, J=8.7,7.7,4.7 \mathrm{~Hz}, 1 \mathrm{H}), 3.89(\mathrm{td}, J=8.4,6.9 \mathrm{~Hz}, 1 \mathrm{H}), 2.22(\mathrm{dq}, J$ $=13.1,7.7 \mathrm{~Hz}, 1 \mathrm{H}), 2.01(\mathrm{ddt}, J=13.1,6.9,4.5 \mathrm{~Hz}, 1 \mathrm{H}), 1.29(\mathrm{t}, J=7.1 \mathrm{~Hz}, 3 \mathrm{H}) ;{ }^{13} \mathrm{C}\left\{{ }^{1} \mathrm{H}\right\} \mathrm{NMR}(101$ MHz, Chloroform- $d$ ) $\delta 170.8,148.0,130.6,123.2,121.9,116.9,112.8,84.7,67.7,62.4,62.1,59.0,32.3$, 14.2; IR (neat): 3365, 2980, 2100, 1730, 1595, 1502, 1257, $1189 \mathrm{~cm}^{-1}$; HRMS (ESI, m/z): calcd for $\mathrm{C}_{14} \mathrm{H}_{17} \mathrm{BrN}_{4} \mathrm{O}_{3} \mathrm{Na}[\mathrm{M}+\mathrm{Na}]^{+}$391.0376, found 391.0378 .

Isomer 2 (more polar): ${ }^{1} \mathrm{H}$ NMR (400 MHz, Chloroform- $d$ ) $\delta 7.04(\mathrm{t}, J=8.0 \mathrm{~Hz}, 1 \mathrm{H}), 6.90$ (ddd, $J=7.9$, $1.8,0.9 \mathrm{~Hz}, 1 \mathrm{H}), 6.81(\mathrm{t}, J=2.1 \mathrm{~Hz}, 1 \mathrm{H}), 6.57(\mathrm{ddd}, J=8.2,2.4,0.9 \mathrm{~Hz}, 1 \mathrm{H}), 4.45(\mathrm{~d}, J=10.1 \mathrm{~Hz}, 1 \mathrm{H})$, 
4.26 - $4.19(\mathrm{~m}, 2 \mathrm{H}), 4.17(\mathrm{dd}, J=10.2,2.0 \mathrm{~Hz}, 1 \mathrm{H}), 4.15-4.06(\mathrm{~m}, 2 \mathrm{H}), 4.10-4.01(\mathrm{~m}, 1 \mathrm{H}), 3.92(\mathrm{dt}$, $J=8.8,7.5 \mathrm{~Hz}, 1 \mathrm{H}), 2.35-2.21(\mathrm{~m}, 1 \mathrm{H}), 2.13-2.00(\mathrm{~m}, 1 \mathrm{H}), 1.27(\mathrm{t}, J=7.2 \mathrm{~Hz}, 3 \mathrm{H}) ;{ }^{13} \mathrm{C}\left\{{ }^{1} \mathrm{H}\right\} \mathrm{NMR}$ (101 MHz, Chloroform- $d$ ) $\delta 171.0,148.3,130.7,123.4,121.9,116.8,112.5,83.6,68.0,62.1,61.9,57.5$, 32.2, 14.2; IR (neat): 3376, 2979, 2100, 1732, 1593, 1499, 1258, $1193 \mathrm{~cm}^{-1}$; HRMS (ESI, m/z): calcd for $\mathrm{C}_{14} \mathrm{H}_{17} \mathrm{BrN}_{4} \mathrm{O}_{3} \mathrm{Na}[\mathrm{M}+\mathrm{Na}]^{+}$391.0376, found 391.0366.

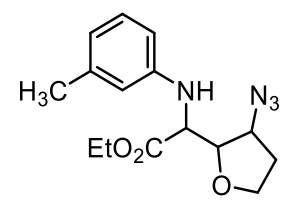

Ethyl 2-(3-azidotetrahydrofuran-2-yl)-2-(m-tolylamino)acetate (4ja): compound 4ja was isolated through a silica gel flash column (PE: EtOAc $=40: 1)$ as yellow oil (51 mg, 84\% yield, $d r=1.2)$.

Isomer 1 (less polar): ${ }^{1} \mathrm{H}$ NMR (400 MHz, Chloroform- $d$ ) $\delta 7.13-7.05(\mathrm{~m}, 1 \mathrm{H}), 6.62$ (ddt, $J=7.5,1.7$, $0.8 \mathrm{~Hz}, 1 \mathrm{H}), 6.59-6.52(\mathrm{~m}, 2 \mathrm{H}), 4.38(\mathrm{~d}, J=8.6 \mathrm{~Hz}, 1 \mathrm{H}), 4.24(\mathrm{q}, J=7.1 \mathrm{~Hz}, 2 \mathrm{H}), 4.21-4.14(\mathrm{~m}, 2 \mathrm{H})$, $4.10(\mathrm{dd}, J=5.3,4.1 \mathrm{~Hz}, 1 \mathrm{H}), 4.00(\mathrm{ddd}, J=8.7,7.8,4.5 \mathrm{~Hz}, 1 \mathrm{H}), 3.90(\mathrm{td}, J=8.4,6.8 \mathrm{~Hz}, 1 \mathrm{H}), 2.29$ (s, $3 \mathrm{H}), 2.28-2.19(\mathrm{~m}, 1 \mathrm{H}), 2.07-1.95(\mathrm{~m}, 1 \mathrm{H}), 1.28(\mathrm{t}, J=7.1 \mathrm{~Hz}, 3 \mathrm{H}) ;{ }^{13} \mathrm{C}\left\{{ }^{1} \mathrm{H}\right\} \mathrm{NMR}(101 \mathrm{MHz}$, Chloroform- $d$ ) $\delta 171.4,146.6,139.2,129.2,120.0,115.0,111.2,84.9,67.7,62.8,61.8,59.4,32.2,21.6$, 14.2; IR (neat): 3360, 2980, 2098, 1731, 1606, 1511, 1258, $1180 \mathrm{~cm}^{-1}$; HRMS (ESI, m/z): calcd for $\mathrm{C}_{15} \mathrm{H}_{20} \mathrm{~N}_{4} \mathrm{O}_{3} \mathrm{Na}[\mathrm{M}+\mathrm{Na}]^{+} 327.1428$, found 327.1433 .

Isomer 2 (more polar): ${ }^{1} \mathrm{H}$ NMR (400 MHz, Chloroform- $d$ ) $\delta 7.09(\mathrm{t}, J=7.7 \mathrm{~Hz}, 1 \mathrm{H}), 6.62$ (ddt, $J=7.5$, $1.7,0.8 \mathrm{~Hz}, 1 \mathrm{H}), 6.54-6.47(\mathrm{~m}, 2 \mathrm{H}), 4.36(\mathrm{~d}, J=10.2 \mathrm{~Hz}, 1 \mathrm{H}), 4.26-4.19(\mathrm{~m}, 3 \mathrm{H}), 4.18-4.11(\mathrm{~m}, 2 \mathrm{H})$, 4.06 (ddd, $J=8.7,7.5,5.1 \mathrm{~Hz}, 1 \mathrm{H}), 3.92(\mathrm{dt}, J=8.7,7.4 \mathrm{~Hz}, 1 \mathrm{H}), 2.35-2.23(\mathrm{~m}, 1 \mathrm{H}), 2.28(\mathrm{~s}, 3 \mathrm{H}), 2.05$ $(\mathrm{ddt}, J=12.6,7.3,5.1 \mathrm{~Hz}, 1 \mathrm{H}), 1.26(\mathrm{t}, J=7.1 \mathrm{~Hz}, 3 \mathrm{H}) ;{ }^{13} \mathrm{C}\left\{{ }^{1} \mathrm{H}\right\} \mathrm{NMR}(101 \mathrm{MHz}$, Chloroform- $d$ ) $\delta 171.5$, 147.0, 139.3, 129.3, 120.0, 114.8, 111.0, 83.9, 68.0, 62.3, 61.7, 57.8, 32.3, 21.6, 14.2; IR (neat): 3376, 2980, 2099, 1740, 1605, 1510, 1260, $1179 \mathrm{~cm}^{-1}$; HRMS (ESI, m/z): calcd for $\mathrm{C}_{15} \mathrm{H}_{20} \mathrm{~N}_{4} \mathrm{O}_{3} \mathrm{Na}[\mathrm{M}+\mathrm{Na}]^{+}$ 327.1428 , found 327.1423 .

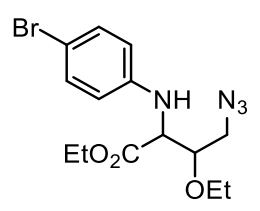

Ethyl 4-azido-2-((4-bromophenyl)amino)-3-ethoxybutanoate (4db): compound $4 \mathrm{db}$ was isolated through a silica gel flash column $(\mathrm{PE}:$ EtOAc $=40: 1)$ as yellow oil (58 mg, 78\% yield, $d r=1.2)$.

Isomer 1 (less polar): ${ }^{1} \mathrm{H}$ NMR (400 MHz, Chloroform- $d$ ) $\delta 7.30-7.21(\mathrm{~m}, 2 \mathrm{H}), 6.57-6.48(\mathrm{~m}, 2 \mathrm{H})$, $4.42(\mathrm{~d}, J=10.5 \mathrm{~Hz}, 1 \mathrm{H}), 4.19(\mathrm{q}, J=7.1 \mathrm{~Hz}, 2 \mathrm{H}), 4.08(\mathrm{dd}, J=10.5,2.2 \mathrm{~Hz}, 1 \mathrm{H}), 3.98(\mathrm{td}, J=6.6,2.2$ $\mathrm{Hz}, 1 \mathrm{H}), 3.67(\mathrm{dq}, J=9.2,7.0 \mathrm{~Hz}, 1 \mathrm{H}), 3.59-3.50(\mathrm{~m}, 2 \mathrm{H}), 3.36(\mathrm{dd}, J=12.4,6.6 \mathrm{~Hz}, 1 \mathrm{H}), 1.25(\mathrm{t}, J=$ $7.1 \mathrm{~Hz}, 3 \mathrm{H}), 1.19(\mathrm{t}, J=7.0 \mathrm{~Hz}, 3 \mathrm{H}) ;{ }^{13} \mathrm{C}\left\{{ }^{1} \mathrm{H}\right\} \mathrm{NMR}(101 \mathrm{MHz}$, Chloroform- $d$ ) $\delta 171.7,146.2,132.0$, 
115.5, 110.5, 78.7, 67.4, 61.6, 57.8, 51.7, 15.4, 14.2; IR (neat): 3382, 2978, 2931, 2097, 1738, 1594, 1496, 1275, $1193 \mathrm{~cm}^{-1}$; HRMS (ESI, m/z): calcd for $\mathrm{C}_{14} \mathrm{H}_{19} \mathrm{BrN}_{4} \mathrm{O}_{3} \mathrm{Na}[\mathrm{M}+\mathrm{Na}]^{+}$393.0533, found 393.0518.

Isomer 2 (more polar): ${ }^{1} \mathrm{H}$ NMR (400 MHz, Chloroform- $d$ ) $\delta 7.31-7.22(\mathrm{~m}, 2 \mathrm{H}), 6.60-6.51(\mathrm{~m}, 2 \mathrm{H})$, $4.35(\mathrm{~d}, J=8.7 \mathrm{~Hz}, 1 \mathrm{H}), 4.27-4.21(\mathrm{~m}, 2 \mathrm{H}), 4.21-4.13(\mathrm{~m}, 1 \mathrm{H}), 3.75-3.65(\mathrm{~m}, 2 \mathrm{H}), 3.59(\mathrm{dq}, J=9.2$, $7.0 \mathrm{~Hz}, 1 \mathrm{H}), 3.52-3.47(\mathrm{~m}, 2 \mathrm{H}), 1.28(\mathrm{t}, J=7.1 \mathrm{~Hz}, 3 \mathrm{H}), 1.20(\mathrm{t}, J=7.0 \mathrm{~Hz}, 3 \mathrm{H}) ;{ }^{13} \mathrm{C}\left\{{ }^{1} \mathrm{H}\right\} \mathrm{NMR}(101$ MHz, Chloroform- $d$ ) $\delta 171.3,145.4,132.1,115.6,110.7,79.4,67.0,61.7,58.5,51.2,15.4,14.2$; IR (neat): 3373, 2978, 2931, 2097, 1729, 1593, 1489, 1284, $1180 \mathrm{~cm}^{-1}$; HRMS (ESI, m/z): calcd for $\mathrm{C}_{14} \mathrm{H}_{19} \mathrm{BrN}_{4} \mathrm{O}_{3} \mathrm{Na}[\mathrm{M}+\mathrm{Na}]^{+}$393.0533, found 393.0503.

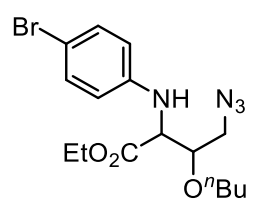

Ethyl 4-azido-2-((4-bromophenyl)amino)-3-butoxybutanoate (4dc): compound $4 \mathrm{dc}$ was isolated through a silica gel flash column $(\mathrm{PE}:$ EtOAc $=30: 1)$ as yellow oil $(60 \mathrm{mg}, 75 \%$ yield, $d r=1.2)$.

Isomer 1 (less polar): ${ }^{1} \mathrm{H}$ NMR (600 MHz, Chloroform- $d$ ) $\delta 7.31-7.23(\mathrm{~m}, 2 \mathrm{H}), 6.56-6.49(\mathrm{~m}, 2 \mathrm{H})$, $4.40(\mathrm{~d}, J=10.6 \mathrm{~Hz}, 1 \mathrm{H}), 4.24-4.15(\mathrm{~m}, 2 \mathrm{H}), 4.09$ (dd, $J=10.6,2.3 \mathrm{~Hz}, 1 \mathrm{H}), 3.97$ (dd, $J=6.6,2.3 \mathrm{~Hz}$, $1 \mathrm{H}), 3.61(\mathrm{dt}, J=9.1,6.4 \mathrm{~Hz}, 1 \mathrm{H}), 3.55(\mathrm{dd}, J=12.4,6.4 \mathrm{~Hz}, 1 \mathrm{H}), 3.47(\mathrm{dt}, J=9.0,6.6 \mathrm{~Hz}, 1 \mathrm{H}), 3.38$ $(\mathrm{dd}, J=12.4,6.8 \mathrm{~Hz}, 1 \mathrm{H}), 1.59-1.49(\mathrm{~m}, 2 \mathrm{H}), 1.40-1.30(\mathrm{~m}, 2 \mathrm{H}), 1.25(\mathrm{t}, J=7.1 \mathrm{~Hz}, 3 \mathrm{H}), 0.91(\mathrm{t}, J=$ $7.4 \mathrm{~Hz}, 3 \mathrm{H}) ;{ }^{13} \mathrm{C}\left\{{ }^{1} \mathrm{H}\right\} \mathrm{NMR}(151 \mathrm{MHz}$, Chloroform-d) $\delta 171.7,146.3,132.0,115.5,110.4,78.9,71.7$, 61.6, 57.8, 51.6, 32.0, 19.1, 14.2, 13.8; IR (neat): 3388, 2958, 2932, 2871, 2098, 1740, 1594, 1497, 1275 , $1193 \mathrm{~cm}^{-1}$; HRMS (ESI, m/z): calcd for $\mathrm{C}_{16} \mathrm{H}_{23} \mathrm{BrN}_{4} \mathrm{O}_{3} \mathrm{Na}[\mathrm{M}+\mathrm{Na}]^{+} 421.0846$, found 421.0836 .

Isomer 2 (more polar): ${ }^{1} \mathrm{H}$ NMR (600 MHz, Chloroform- $d$ ) $\delta 7.29-7.23(\mathrm{~m}, 2 \mathrm{H}), 6.58-6.52(\mathrm{~m}, 2 \mathrm{H})$, $4.34(\mathrm{~d}, J=8.8 \mathrm{~Hz}, 1 \mathrm{H}), 4.22(\mathrm{q}, J=7.1 \mathrm{~Hz}, 2 \mathrm{H}), 4.18(\mathrm{dd}, J=8.4,5.6 \mathrm{~Hz}, 1 \mathrm{H}), 3.74-3.67(\mathrm{~m}, 1 \mathrm{H})$, $3.63(\mathrm{dt}, J=9.0,6.4 \mathrm{~Hz}, 1 \mathrm{H}), 3.55-3.45(\mathrm{~m}, 3 \mathrm{H}), 1.59-1.50(\mathrm{~m}, 2 \mathrm{H}), 1.43-1.32(\mathrm{~m}, 2 \mathrm{H}), 1.28(\mathrm{t}, J=$ $7.1 \mathrm{~Hz}, 3 \mathrm{H}), 0.91(\mathrm{t}, J=7.4 \mathrm{~Hz}, 3 \mathrm{H}) ;{ }^{13} \mathrm{C}\left\{{ }^{1} \mathrm{H}\right\} \mathrm{NMR}(151 \mathrm{MHz}$, Chloroform-d) $\delta 171.4,145.4,132.1$, 115.6, 110.7, 79.6, 71.3, 61.7, 58.5, 51.1, 32.0, 19.1, 14.2, 13.8; IR (neat): 3368, 2958, 2932, 2871, 2099 , 1730, 1593, 1489, 1286, $1178 \mathrm{~cm}^{-1}$; HRMS (ESI, m/z): calcd for $\mathrm{C}_{16} \mathrm{H}_{23} \mathrm{BrN}_{4} \mathrm{O}_{3} \mathrm{Na}[\mathrm{M}+\mathrm{Na}]^{+} 421.0846$, found 421.0826 .

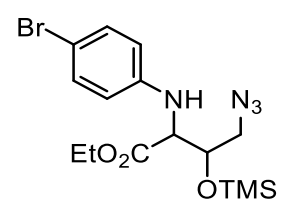

Ethyl 4-azido-2-((4-bromophenyl)amino)-3-((trimethylsilyl)oxy)butanoate (4dd): compound 4dd was isolated through a silica gel flash column (PE: EtOAc $=100: 1)$ as yellow oil (42 mg, 51\% yield, $d r=1.3$ ). 
Isomer 1 (less polar): ${ }^{1} \mathrm{H}$ NMR (600 MHz, Chloroform- $d$ ) $\delta 7.28-7.23(\mathrm{~m}, 2 \mathrm{H}), 6.56-6.50(\mathrm{~m}, 2 \mathrm{H})$, $4.41(\mathrm{~d}, J=10.9 \mathrm{~Hz}, 1 \mathrm{H}), 4.28(\mathrm{td}, J=6.8,1.9 \mathrm{~Hz}, 1 \mathrm{H}), 4.22-4.11(\mathrm{~m}, 2 \mathrm{H}), 4.08(\mathrm{dd}, J=10.9,1.9 \mathrm{~Hz}$, $1 \mathrm{H}), 3.46$ (dd, $J=12.1,7.1 \mathrm{~Hz}, 1 \mathrm{H}), 3.39$ (dd, $J=12.1,6.4 \mathrm{~Hz}, 1 \mathrm{H}), 1.24$ (t, $J=7.1 \mathrm{~Hz}, 3 \mathrm{H}), 0.13$ (s, $9 \mathrm{H}) ;{ }^{13} \mathrm{C}\left\{{ }^{1} \mathrm{H}\right\} \mathrm{NMR}(151 \mathrm{MHz}$, Chloroform- $d) \delta 171.6,146.2,132.0,115.5,110.4,71.9,61.6,58.6,53.9$, 14.2, 0.0; IR (neat): 3390, 2958, 2101, 1745, 1595, 1497, 1252, $1193 \mathrm{~cm}^{-1}$; HRMS (ESI, m/z): calcd for $\mathrm{C}_{15} \mathrm{H}_{23} \mathrm{BrN}_{4} \mathrm{O}_{3} \mathrm{SiNa}[\mathrm{M}+\mathrm{Na}]^{+}$437.0615, found 437.0606.

Isomer 2 (more polar): ${ }^{1} \mathrm{H}$ NMR (400 MHz, Chloroform- $d$ ) $\delta 7.31-7.23(\mathrm{~m}, 2 \mathrm{H}), 6.58-6.50(\mathrm{~m}, 2 \mathrm{H})$, $4.35(\mathrm{~d}, J=8.9 \mathrm{~Hz}, 1 \mathrm{H}), 4.22(\mathrm{q}, J=7.2 \mathrm{~Hz}, 2 \mathrm{H}), 4.13(\mathrm{dd}, J=8.8,4.9 \mathrm{~Hz}, 1 \mathrm{H}), 4.03(\mathrm{dt}, J=5.8,5.0 \mathrm{~Hz}$, 1H), 3.51 (dd, $J=12.6,5.0 \mathrm{~Hz}, 1 \mathrm{H}), 3.44$ (dd, $J=12.6,5.9 \mathrm{~Hz}, 1 \mathrm{H}), 1.28$ (t, $J=7.1 \mathrm{~Hz}, 3 \mathrm{H}), 0.14$ (s, $9 \mathrm{H}) ;{ }^{13} \mathrm{C}\left\{{ }^{1} \mathrm{H}\right\} \mathrm{NMR}(101 \mathrm{MHz}$, Chloroform- $d$ ) $\delta 171.0,145.2,132.1,115.4,110.5,72.7,61.6,59.6,54.0$, 14.2, 0.1; IR (neat): 3390, 2958, 2099, 1732, 1594, 1497, 1251, $1179 \mathrm{~cm}^{-1}$; HRMS (ESI, m/z): calcd for $\mathrm{C}_{15} \mathrm{H}_{23} \mathrm{BrN}_{4} \mathrm{O}_{3} \mathrm{SiNa}[\mathrm{M}+\mathrm{Na}]^{+}$437.0615, found 437.0627 .

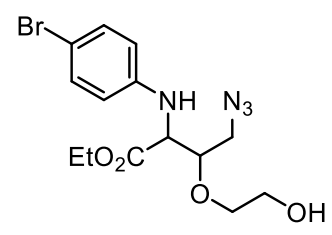

Ethyl 4-azido-2-((4-bromophenyl)amino)-3-(2-hydroxyethoxy)butanoate (4de): compound 4de was isolated through a silica gel flash column (PE: Acetone $=5: 1)$ as yellow oil (43 mg, 56\% yield, $d r=1.5)$. ${ }^{1} \mathrm{H}$ NMR (600 MHz, Chloroform- $d$ ) (mixture of 2 diastereomers, 1.5:1) $\delta 7.30-7.23(\mathrm{~m}, 2 \mathrm{H}$, minor + $2 \mathrm{H}$, major), $6.60-6.54$ (m, 2H, minor), $6.57-6.51$ (m, 2H, major), $\delta 4.61$ (brs, $1 \mathrm{H}$, major), $\delta 4.28-4.20$ $(\mathrm{m}, 3 \mathrm{H}$, minor $+2 \mathrm{H}$, major), $4.13(\mathrm{~d}, J=2.5 \mathrm{~Hz}, 1 \mathrm{H}$, major), $\delta 4.02(\mathrm{td}, J=6.5,2.4 \mathrm{~Hz}, 1 \mathrm{H}$, major), $\delta$ $3.88-3.77(\mathrm{~m}, 1 \mathrm{H}$, minor $+2 \mathrm{H}$, major $), \delta 3.77-3.65(\mathrm{~m}, 3 \mathrm{H}$, minor $+2 \mathrm{H}$, major $), \delta 3.64-3.60(\mathrm{~m}, 1 \mathrm{H}$, minor $+1 \mathrm{H}$, major), $\delta 3.58-3.53(\mathrm{~m}, 1 \mathrm{H}$, major $+1 \mathrm{H}$, minor), $3.46(\mathrm{dd}, \mathrm{J}=12.5,6.3 \mathrm{~Hz}, 1 \mathrm{H}$, major), $\delta$ 2.71 (br, $1 \mathrm{H}$, minor $+1 \mathrm{H}$, major), $1.29(\mathrm{t}, J=7.2 \mathrm{~Hz}, 1 \mathrm{H}$, minor), $1.28(\mathrm{t}, J=7.1 \mathrm{~Hz}, 1 \mathrm{H}$, major); ${ }^{13} \mathrm{C}\left\{{ }^{1} \mathrm{H}\right\} \mathrm{NMR}$ (151 MHz, Chloroform- $d$ ) (mixture of 2 diastereomers) $\delta 172.0$ (major), 171.4 (minor), 146.0 (major), 145.2 (minor), 132.2 (minor), 132.1 (major), 115.6 (minor), 115.5 (major), 110.8 (minor), 110.6 (major), 79.9 (minor), 79.4 (major), 73.4 (major), 73.2 (minor), 62.0 (minor), 61.9(major + minor), 61.8 (major), 58.6 (minor), 57.8 (major), 51.8 (major), 51.5 (minor), 14.22 (minor), 14.20 (major); IR (neat): 3372, 2931, 2099, 1728, 1594, 1489, 1264, $1194 \mathrm{~cm}^{-1}$; HRMS (ESI, m/z): calcd for $\mathrm{C}_{14} \mathrm{H}_{19} \mathrm{BrN}_{4} \mathrm{O}_{4} \mathrm{Na}[\mathrm{M}+\mathrm{Na}]^{+} 409.0482$, found 409.0463 .

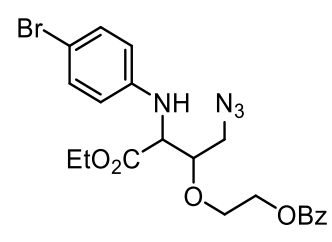


2-((1-Azido-3-((4-bromophenyl)amino)-4-ethoxy-4-oxobutan-2-yl)oxy)ethyl benzoate (4df): compound 4df was isolated through a silica gel flash column $(\mathrm{PE}$ : EtOAc $=5: 1)$ as yellow oil $(79 \mathrm{mg}, 81 \%$ yield, $d r$ $=1.3)$.

Isomer 1 (less polar): ${ }^{1} \mathrm{H}$ NMR (400 MHz, Chloroform- $d$ ) $\delta 8.07(\mathrm{~d}, J=7.7 \mathrm{~Hz}, 2 \mathrm{H}), 7.58(\mathrm{t}, J=7.5 \mathrm{~Hz}$, 1H), $7.45(\mathrm{t}, J=7.7 \mathrm{~Hz}, 2 \mathrm{H}), 7.24(\mathrm{~d}, J=8.6 \mathrm{~Hz}, 2 \mathrm{H}), 6.50(\mathrm{~d}, J=8.3 \mathrm{~Hz}, 2 \mathrm{H}), 4.50-4.39(\mathrm{~m}, 3 \mathrm{H}), 4.22$ - $4.04(\mathrm{~m}, 4 \mathrm{H}), 3.96(\mathrm{dt}, J=9.6,4.5 \mathrm{~Hz}, 1 \mathrm{H}), 3.86(\mathrm{dt}, J=10.5,4.7 \mathrm{~Hz}, 1 \mathrm{H}), 3.61(\mathrm{dd}, J=12.5,6.6 \mathrm{~Hz}$, $1 \mathrm{H}), 3.42(\mathrm{dd}, J=12.5,6.3 \mathrm{~Hz}, 1 \mathrm{H}), 1.19(\mathrm{t}, J=7.2 \mathrm{~Hz}, 3 \mathrm{H}) ;{ }^{13} \mathrm{C}\left\{{ }^{1} \mathrm{H}\right\} \mathrm{NMR}(101 \mathrm{MHz}$, Chloroform- $d) \delta$ $171.5,166.5,146.1,133.2,132.0,129.9,129.7,128.5,115.5,110.6,79.4,69.7,63.9,61.7,57.8,51.8$, 14.2; IR (neat): 3382, 2935, 2101, 1730, 1717, 1594, 1498, 1272, $1195 \mathrm{~cm}^{-1}$; HRMS (ESI, m/z): calcd for $\mathrm{C}_{21} \mathrm{H}_{23} \mathrm{BrN}_{4} \mathrm{O}_{5} \mathrm{Na}[\mathrm{M}+\mathrm{Na}]^{+}$513.0744, found 513.0745.

Isomer 2 (more polar): ${ }^{1} \mathrm{H}$ NMR (400 MHz, Chloroform- $d$ ) $\delta 8.06(\mathrm{~d}, J=7.7 \mathrm{~Hz}, 2 \mathrm{H}), 7.58(\mathrm{t}, J=7.4 \mathrm{~Hz}$, 1H), $7.44(\mathrm{t}, J=7.7 \mathrm{~Hz}, 2 \mathrm{H}), 7.21(\mathrm{~d}, J=8.4 \mathrm{~Hz}, 2 \mathrm{H}), 6.50(\mathrm{~d}, J=8.4 \mathrm{~Hz}, 2 \mathrm{H}), 4.52-4.41(\mathrm{~m}, 3 \mathrm{H}), 4.24$ - $4.13(\mathrm{~m}, 3 \mathrm{H}), 4.05-3.97(\mathrm{~m}, 1 \mathrm{H}), 3.96-3.89(\mathrm{~m}, 1 \mathrm{H}), 3.88-3.80(\mathrm{~m}, 1 \mathrm{H}), 3.61-3.52(\mathrm{~m}, 2 \mathrm{H}), 1.22$ $(\mathrm{t}, J=7.2 \mathrm{~Hz}, 3 \mathrm{H}) ;{ }^{13} \mathrm{C}\left\{{ }^{1} \mathrm{H}\right\} \mathrm{NMR}(101 \mathrm{MHz}$, Chloroform- $d$ ) $\delta 170.8,166.5,145.2,133.2,132.1,129.9$, 129.7, 128.4, 115.5, 110.8, 80.1, 69.6, 64.0, 61.8, 58.6, 51.5, 14.2; IR (neat): 3371, 2979, 2099, 1716, 1593, 1489, 1270, $1178 \mathrm{~cm}^{-1}$; HRMS (ESI, m/z): calcd for $\mathrm{C}_{21} \mathrm{H}_{23} \mathrm{BrN}_{4} \mathrm{O}_{5} \mathrm{Na}[\mathrm{M}+\mathrm{Na}]^{+} 513.0744$, found 513.0734 .

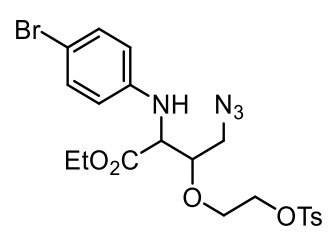

Ethyl 4-azido-2-((4-bromophenyl)amino)-3-(2-(tosyloxy)ethoxy)butanoate (4dg): compound 4dg was isolated through a silica gel flash column $(\mathrm{PE}:$ EtOAc $=4: 1)$ as yellow oil $(84 \mathrm{mg}, 78 \%$ yield, $d r=1.3)$.

Isomer 1 (less polar): ${ }^{1} \mathrm{H}$ NMR (400 MHz, Chloroform- $d$ ) $\delta 7.85-7.77(\mathrm{~m}, 2 \mathrm{H}), 7.35(\mathrm{~d}, J=8.0 \mathrm{~Hz}, 2 \mathrm{H})$, $7.28-7.20(\mathrm{~m}, 2 \mathrm{H}), 6.56-6.43(\mathrm{~m}, 2 \mathrm{H}), 4.37(\mathrm{~d}, J=10.5 \mathrm{~Hz}, 1 \mathrm{H}), 4.23-4.13(\mathrm{~m}, 2 \mathrm{H}), 4.12(\mathrm{t}, J=4.6$ $\mathrm{Hz}, 2 \mathrm{H}), 4.07$ (dd, $J=10.6,2.4 \mathrm{~Hz}, 1 \mathrm{H}), 3.99(\mathrm{td}, J=6.5,2.4 \mathrm{~Hz}, 1 \mathrm{H}), 3.82$ (dt, $J=11.4,4.4 \mathrm{~Hz}, 1 \mathrm{H})$, $3.73(\mathrm{dt}, J=11.3,4.7 \mathrm{~Hz}, 1 \mathrm{H}), 3.51(\mathrm{dd}, J=12.5,6.5 \mathrm{~Hz}, 1 \mathrm{H}), 3.38(\mathrm{dd}, J=12.5,6.5 \mathrm{~Hz}, 1 \mathrm{H}), 2.45(\mathrm{~s}$, $3 \mathrm{H}), 1.23(\mathrm{t}, J=7.1 \mathrm{~Hz}, 3 \mathrm{H}) ;{ }^{13} \mathrm{C}\left\{{ }^{1} \mathrm{H}\right\} \mathrm{NMR}(101 \mathrm{MHz}$, Chloroform- $d$ ) $\delta 171.4,146.1,145.1,132.9,132.1$, 129.9, 128.0, 115.5, 110.6, 79.7, 69.3, 68.8, 61.8, 57.8, 51.6, 21.7, 14.2; IR (neat): 3388, 2981, 2099, 1740, 1597, 1496, 1292, $1191 \mathrm{~cm}^{-1}$; HRMS (ESI, m/z): calcd for $\mathrm{C}_{21} \mathrm{H}_{25} \mathrm{BrN}_{4} \mathrm{O}_{6} \mathrm{SNa}\left[\mathrm{M}+\mathrm{Na}^{+} 563.0570\right.$, found 563.0562 .

Isomer 2 (more polar): ${ }^{1} \mathrm{H}$ NMR (400 MHz, Chloroform- $d$ ) $\delta 7.79(\mathrm{~d}, J=7.9 \mathrm{~Hz}, 2 \mathrm{H}), 7.34(\mathrm{~d}, J=8.0 \mathrm{~Hz}$, $2 \mathrm{H}), 7.25(\mathrm{~d}, J=8.5 \mathrm{~Hz}, 2 \mathrm{H}), 6.54(\mathrm{~d}, J=8.4 \mathrm{~Hz}, 2 \mathrm{H}), 4.48(\mathrm{br}, 1 \mathrm{H}), 4.22(\mathrm{q}, J=7.1 \mathrm{~Hz}, 2 \mathrm{H}), 4.17-4.11$ $(\mathrm{m}, 3 \mathrm{H}), 3.87(\mathrm{dt}, J=11.5,4.2 \mathrm{~Hz}, 1 \mathrm{H}), 3.84-3.70(\mathrm{~m}, 2 \mathrm{H}), 3.57-3.40(\mathrm{~m}, 2 \mathrm{H}), 2.45(\mathrm{~s}, 3 \mathrm{H}), 1.26(\mathrm{t}, J$ 
$=7.1 \mathrm{~Hz}, 3 \mathrm{H}) ;{ }^{13} \mathrm{C}\left\{{ }^{1} \mathrm{H}\right\} \mathrm{NMR}(101 \mathrm{MHz}$, Chloroform- $d) \delta 170.6,145.2,145.0,132.8,132.1,129.9,128.0$, 115.6, 110.8, 80.3, 69.4, 68.8, 61.9, 58.5, 51.5, 21.7, 14.2; IR (neat): 3376, 2981, 2101, 1732, 1594, 1489, 1292, $1187 \mathrm{~cm}^{-1}$; HRMS (ESI, m/z): calcd for $\mathrm{C}_{21} \mathrm{H}_{25} \mathrm{BrN}_{4} \mathrm{O}_{6} \mathrm{SNa}[\mathrm{M}+\mathrm{Na}]^{+}$563.0570, found 563.0557.

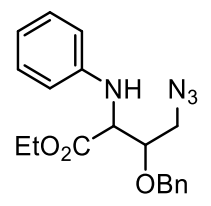

Ethyl 4-azido-3-(benzyloxy)-2-(phenylamino)butanoate (4bh): compound $4 \mathrm{bh}$ was isolated through a silica gel flash column (PE: EtOAc $=20: 1)$ as yellow oil $(43 \mathrm{mg}, 61 \%$ yield, $d r=1.2)$.

Isomer 1 (less polar): ${ }^{1} \mathrm{H}$ NMR (400 MHz, Chloroform- $d$ ) $\delta 7.43-7.29(\mathrm{~m}, 5 \mathrm{H}), 7.24-7.13(\mathrm{~m}, 2 \mathrm{H})$, $6.82-6.73(\mathrm{~m}, 1 \mathrm{H}), 6.68-6.60(\mathrm{~m}, 2 \mathrm{H}), 4.68(\mathrm{~d}, J=11.3 \mathrm{~Hz}, 1 \mathrm{H}), 4.58(\mathrm{~d}, J=11.3 \mathrm{~Hz}, 1 \mathrm{H}), \delta 4.43(\mathrm{br}$, $1 \mathrm{H}), 4.25-4.05(\mathrm{~m}, 4 \mathrm{H}), 3.61(\mathrm{dd}, J=12.4,6.4 \mathrm{~Hz}, 1 \mathrm{H}), 3.50(\mathrm{dd}, J=12.4,6.7 \mathrm{~Hz}, 1 \mathrm{H}), 1.21(\mathrm{t}, J=7.1$ $\mathrm{Hz}, 3 \mathrm{H}) ;{ }^{13} \mathrm{C}\left\{{ }^{1} \mathrm{H}\right\} \mathrm{NMR}(101 \mathrm{MHz}$, Chloroform- $d$ ) $\delta 171.9,147.0,137.2,129.3,128.5,128.2,128.2,118.8$, 113.9, 78.2, 73.5, 61.5, 57.8, 51.7, 14.2; IR (neat): 3386, 2981, 2101, 1743, 1602, 1506, 1276, $1194 \mathrm{~cm}^{-}$ 1; HRMS (ESI, m/z): calcd for $\mathrm{C}_{19} \mathrm{H}_{22} \mathrm{~N}_{4} \mathrm{O}_{3} \mathrm{Na}[\mathrm{M}+\mathrm{Na}]^{+} 377.1584$, found 377.1573.

Isomer 2 (more polar): ${ }^{1} \mathrm{H}$ NMR (400 MHz, Chloroform- $d$ ) $\delta 7.38-7.30(\mathrm{~m}, 5 \mathrm{H}), 7.22-7.15(\mathrm{~m}, 2 \mathrm{H})$, $6.78(\mathrm{tt}, J=7.2,1.1 \mathrm{~Hz}, 1 \mathrm{H}), 6.68-6.62(\mathrm{~m}, 2 \mathrm{H}), 4.72(\mathrm{~d}, J=11.5 \mathrm{~Hz}, 1 \mathrm{H}), 4.63(\mathrm{~d}, J=11.5 \mathrm{~Hz}, 1 \mathrm{H})$, $4.28(\mathrm{~d}, J=5.4 \mathrm{~Hz}, 1 \mathrm{H}), 4.20(\mathrm{q}, J=7.1 \mathrm{~Hz}, 2 \mathrm{H}), 3.89(\mathrm{dt}, J=5.2 \mathrm{~Hz}, 1 \mathrm{H}), 3.60-3.53(\mathrm{~m}, 2 \mathrm{H}), 1.24(\mathrm{t}$, $J=7.2 \mathrm{~Hz}, 3 \mathrm{H}) ;{ }^{13} \mathrm{C}\left\{{ }^{1} \mathrm{H}\right\} \mathrm{NMR}(101 \mathrm{MHz}$, Chloroform- $d) \delta 171.6,146.2,137.3,129.4,128.5,128.1$, 128.0, 119.0, 114.0, 79.2, 73.5, 61.6, 58.5, 51.6, 14.2; IR (neat): 3371, 2933, 2099, 1729, 1601, 1497 , 1260, $1181 \mathrm{~cm}^{-1}$; HRMS (ESI, m/z): calcd for $\mathrm{C}_{19} \mathrm{H}_{22} \mathrm{~N}_{4} \mathrm{O}_{3} \mathrm{Na}[\mathrm{M}+\mathrm{Na}]^{+} 377.1584$, found 377.1563 .

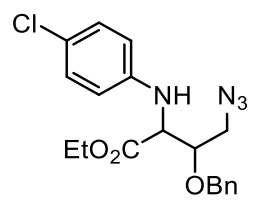

Ethyl 4-azido-3-(benzyloxy)-2-((4-chlorophenyl)amino)butanoate (4ch): compound 4ch was isolated through a silica gel flash column $(\mathrm{PE}:$ EtOAc $=20: 1)$ as yellow oil (63 mg, 81\% yield, $d r=1.3)$.

Isomer 1 (less polar): ${ }^{1} \mathrm{H}$ NMR (600 MHz, Chloroform- $d$ ) $\delta 7.41-7.30(\mathrm{~m}, 5 \mathrm{H}), 7.12(\mathrm{~d}, J=8.5 \mathrm{~Hz}, 2 \mathrm{H})$, $6.56(\mathrm{~d}, J=8.6 \mathrm{~Hz}, 2 \mathrm{H}), 4.67(\mathrm{~d}, J=11.3 \mathrm{~Hz}, 1 \mathrm{H}), 4.55(\mathrm{~d}, J=11.3 \mathrm{~Hz}, 1 \mathrm{H}), 4.43(\mathrm{~d}, J=10.6 \mathrm{~Hz}, 1 \mathrm{H})$, $4.20-4.06(\mathrm{~m}, 4 \mathrm{H}), 3.59(\mathrm{dd}, J=12.4,6.2 \mathrm{~Hz}, 1 \mathrm{H}), 3.48(\mathrm{dd}, J=12.4,6.9 \mathrm{~Hz}, 1 \mathrm{H}), 1.21(\mathrm{t}, J=7.1 \mathrm{~Hz}$, $3 \mathrm{H}) ;{ }^{13} \mathrm{C}\left\{{ }^{1} \mathrm{H}\right\} \mathrm{NMR}(151 \mathrm{MHz}$, Chloroform- $d) \delta 171.6,145.7,137.0,129.2,128.6,128.3,128.2,123.4$, 115.0, 77.9, 73.5, 61.7, 57.9, 51.5, 14.2; IR (neat): 3389, 2981, 2101, 1742, 1600, 1500, 1276, $1196 \mathrm{~cm}^{-}$ 1; HRMS (ESI, m/z): calcd for $\mathrm{C}_{19} \mathrm{H}_{21} \mathrm{ClN}_{4} \mathrm{O}_{3} \mathrm{Na}[\mathrm{M}+\mathrm{Na}]^{+}$411.1194, found 411.1202.

Isomer 2 (more polar): ${ }^{1} \mathrm{H}$ NMR (600 MHz, Chloroform- $d$ ) $\delta 7.39-7.30(\mathrm{~m}, 5 \mathrm{H}), 7.14-7.09(\mathrm{~m}, 2 \mathrm{H})$, $6.57-6.52(\mathrm{~m}, 2 \mathrm{H}), 4.72(\mathrm{~d}, J=11.6 \mathrm{~Hz}, 1 \mathrm{H}), 4.61(\mathrm{~d}, J=11.6 \mathrm{~Hz}, 1 \mathrm{H}), 4.30(\mathrm{~d}, J=9.1 \mathrm{~Hz}, 1 \mathrm{H}), 4.25$ 
- $4.17(\mathrm{~m}, 3 \mathrm{H}), 3.87(\mathrm{dt}, J=5.3 \mathrm{~Hz}, 1 \mathrm{H}), 3.59-3.53(\mathrm{~m}, 2 \mathrm{H}), 1.25(\mathrm{t}, J=7.1 \mathrm{~Hz}, 3 \mathrm{H}) ;{ }^{13} \mathrm{C}\left\{{ }^{1} \mathrm{H}\right\} \mathrm{NMR}$ $(151 \mathrm{MHz}$, Chloroform- $d$ ) $\delta 171.2,144.8,137.2,129.2,128.6,128.2,128.0,123.6,115.1,78.9,73.4$, 61.8, 58.6, 51.4, 14.2; IR (neat): 3374, 2981, 2100, 1731, 1598, 1494, 1260, $1179 \mathrm{~cm}^{-1}$; HRMS (ESI, $\mathrm{m} / \mathrm{z}$ ): calcd for $\mathrm{C}_{19} \mathrm{H}_{21} \mathrm{ClN}_{4} \mathrm{O}_{3} \mathrm{Na}[\mathrm{M}+\mathrm{Na}]^{+} 411.1194$, found 411.1199 .

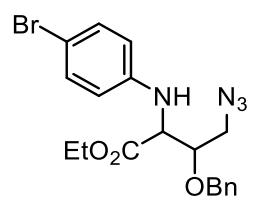

Ethyl 4-azido-3-(benzyloxy)-2-((4-bromophenyl)amino)butanoate (4dh): compound 4dh was isolated through a silica gel flash column $(\mathrm{PE}:$ EtOAc $=20: 1)$ as yellow oil $(62 \mathrm{mg}, 72 \%$ yield, $d r=1.3)$.

Isomer 1 (less polar): ${ }^{1} \mathrm{H}$ NMR (400 MHz, Chloroform- $d$ ) $\delta 7.42-7.29(\mathrm{~m}, 5 \mathrm{H}), 7.28-7.22(\mathrm{~m}, 2 \mathrm{H})$, $6.50(\mathrm{~d}, J=8.3 \mathrm{~Hz}, 2 \mathrm{H}), 4.66(\mathrm{~d}, J=11.3 \mathrm{~Hz}, 1 \mathrm{H}), 4.54(\mathrm{~d}, J=11.3 \mathrm{~Hz}, 1 \mathrm{H}), 4.43(\mathrm{~d}, J=10.5 \mathrm{~Hz}, 1 \mathrm{H})$, $4.23-4.03(\mathrm{~m}, 4 \mathrm{H}), 3.58(\mathrm{dd}, J=12.4,6.2 \mathrm{~Hz}, 1 \mathrm{H}), 3.46(\mathrm{dd}, J=12.4,6.9 \mathrm{~Hz}, 1 \mathrm{H}), 1.20(\mathrm{t}, J=7.1 \mathrm{~Hz}$, $3 \mathrm{H}) ;{ }^{13} \mathrm{C}\left\{{ }^{1} \mathrm{H}\right\} \mathrm{NMR}(101 \mathrm{MHz}$, Chloroform-d) $\delta$ 171.6, 146.2, 137.0, 132.1, 128.6, 128.3, 128.2, 115.5, 110.5, 77.9, 73.5, 61.7, 57.8, 51.5, 14.2; IR (neat): 3389, 2980, 2101, 1739, 1594, 1497, 1276, $1196 \mathrm{~cm}^{-}$ 1; HRMS (ESI, m/z): calcd for $\mathrm{C}_{19} \mathrm{H}_{21} \mathrm{BrN}_{4} \mathrm{O}_{3} \mathrm{Na}[\mathrm{M}+\mathrm{Na}]^{+} 455.0689$, found 455.0695 .

Isomer 2 (more polar): ${ }^{1} \mathrm{H}$ NMR (400 MHz, Chloroform- $d$ ) $\delta 7.42-7.27(\mathrm{~m}, 5 \mathrm{H}), 7.29-7.20$ (m, 2H), $6.55-6.46(\mathrm{~m}, 2 \mathrm{H}), 4.72(\mathrm{~d}, J=11.6 \mathrm{~Hz}, 1 \mathrm{H}), 4.60(\mathrm{~d}, J=11.6 \mathrm{~Hz}, 1 \mathrm{H}), 4.32(\mathrm{~d}, J=9.1 \mathrm{~Hz}, 1 \mathrm{H}), 4.26$ - $4.16(\mathrm{~m}, 3 \mathrm{H}), 3.87(\mathrm{dt}, J=5.3 \mathrm{~Hz}, 1 \mathrm{H}), 3.61-3.49(\mathrm{~m}, 2 \mathrm{H}), 1.25(\mathrm{t}, J=7.1 \mathrm{~Hz}, 3 \mathrm{H}) ;{ }^{13} \mathrm{C}\left\{{ }^{1} \mathrm{H}\right\} \mathrm{NMR}$ (101 MHz, Chloroform- $d$ ) $\delta 171.1,145.2,137.2,132.1,128.5,128.1,128.0,115.5,110.7,78.9$, 73.4, 61.8, 58.5, 51.4, 14.2; IR (neat): 3372, 2980, 2099, 1730, 1593, 1496, 1261, $1180 \mathrm{~cm}^{-1}$; HRMS (ESI, $\mathrm{m} / \mathrm{z}$ ): calcd for $\mathrm{C}_{19} \mathrm{H}_{21} \mathrm{BrN}_{4} \mathrm{O}_{3} \mathrm{Na}[\mathrm{M}+\mathrm{Na}]^{+} 455.0689$, found 455.0678 .

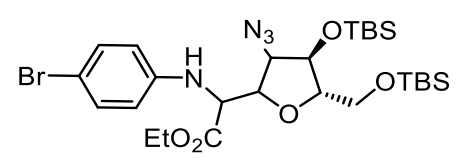

Ethyl 2-((4R,5S)-3-azido-4-((tert-butyldimethylsilyl)oxy)-5-(((tert-butyldimethylsilyl)oxy)methyl)tetrahydrofuran-2-yl)-2-((4-bromophenyl)amino)acetate (4di): compound 4di was isolated through a silica gel flash column $(\mathrm{PE}: \mathrm{EtOAc}=100: 1)$ as yellow oil $(45 \mathrm{mg}, 35 \%$ yield, $d r=1.1)$.

Isomer 1 (less polar): ${ }^{1} \mathrm{H}$ NMR (400 MHz, Chloroform- $d$ ) $\delta 7.29-7.20(\mathrm{~m}, 2 \mathrm{H}), 6.69-6.60(\mathrm{~m}, 2 \mathrm{H})$, $4.53(\mathrm{~d}, J=8.1 \mathrm{~Hz}, 1 \mathrm{H}), 4.35-4.17(\mathrm{~m}, 4 \mathrm{H}), 4.12(\mathrm{dd}, J=7.1,4.7 \mathrm{~Hz}, 1 \mathrm{H}), 3.96(\mathrm{dd}, J=7.2,6.0 \mathrm{~Hz}$, 1H), $3.78(\mathrm{dt}, J=6.4,3.5 \mathrm{~Hz}, 1 \mathrm{H}), 3.72(\mathrm{dd}, J=11.4,3.5 \mathrm{~Hz}, 1 \mathrm{H}), 3.62$ (dd, $J=11.4,3.5 \mathrm{~Hz}, 1 \mathrm{H}), 1.28$ $(\mathrm{t}, J=7.1 \mathrm{~Hz}, 3 \mathrm{H}), 0.97-0.81(\mathrm{~m}, 18 \mathrm{H}), 0.16(\mathrm{~s}, 3 \mathrm{H}), 0.11(\mathrm{~s}, 3 \mathrm{H}), 0.08-0.04(\mathrm{~m}, 6 \mathrm{H}) ;{ }^{13} \mathrm{C}\left\{{ }^{1} \mathrm{H}\right\} \mathrm{NMR}$ $(101 \mathrm{MHz}$, Chloroform- $d$ ) $\delta 170.8,145.9,131.9,115.8,110.7,84.4,81.9,75.8,68.9,62.1,61.9,59.0$, 
25.8, 25.6, 18.3, 17.9, 14.2, -4.8, -4.8, -5.3, -5.5; IR (neat): 3376, 2953, 2928, 2856, 2105, 1743, 1595 , $1497,1252,1188,1144,1098 \mathrm{~cm}^{-1}$.

Isomer 2 (more polar): ${ }^{1} \mathrm{H}$ NMR (600 MHz, Chloroform- $\left.d\right) \delta 7.28(\mathrm{~d}, J=8.6 \mathrm{~Hz}, 2 \mathrm{H}), 6.56(\mathrm{~d}, J=8.7 \mathrm{~Hz}$, $2 \mathrm{H}), \delta 4.51(\mathrm{~d}, J=10.1 \mathrm{~Hz}, 1 \mathrm{H}), 4.46(\mathrm{t}, J=6.9 \mathrm{~Hz}, 1 \mathrm{H}), 4.25-4.19(\mathrm{~m}, 1 \mathrm{H}), 4.17-4.11(\mathrm{~m}, 3 \mathrm{H}), 3.98$ $(\mathrm{dd}, J=8.8,7.2 \mathrm{~Hz}, 1 \mathrm{H}), 3.82(\mathrm{dt}, J=6.1,2.7 \mathrm{~Hz}, 1 \mathrm{H}), 3.77(\mathrm{dd}, J=11.6,2.8 \mathrm{~Hz}, 1 \mathrm{H}), 3.65(\mathrm{dd}, J=11.6$, $2.5 \mathrm{~Hz}, 1 \mathrm{H}), 1.24$ (t, J= 7.1 Hz, 3H), $0.93-0.89$ (m, 18H), 0.17 (s, 3H), 0.12 (s, 3H), 0.05 (s, 3H), 0.03 $(\mathrm{s}, 3 \mathrm{H}) ;{ }^{13} \mathrm{C}\left\{{ }^{1} \mathrm{H}\right\} \mathrm{NMR}(151 \mathrm{MHz}$, Chloroform- $d$ ) $\delta$ 171.1, 146.2, 132.2, 115.6, 110.7, 84.3, 80.7, 75.0, 68.1, 61.8, 61.7, 57.3, 25.8, 25.6, 18.2, 17.9, 14.1, -4.8, -4.9, -5.4, -5.5; IR (neat): 3363, 2953, 2928, 2856, 2105, 1733, 1595, 1498, 1252, 1180, 1147, $1097 \mathrm{~cm}^{-1}$; HRMS (ESI, m/z): calcd for $\mathrm{C}_{27} \mathrm{H}_{47} \mathrm{BrN}_{4} \mathrm{O}_{5} \mathrm{Si}_{2} \mathrm{Na}$ $[\mathrm{M}+\mathrm{Na}]^{+} 665.2161$, found 665.2131 .

\section{Synthesis of silyl enol ether 5}

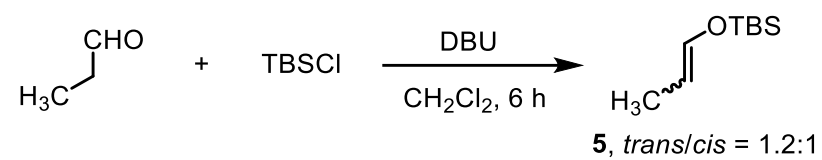

tert-Butyldimethylchlorosilane $(3.62 \mathrm{~g}, 24 \mathrm{mmol})$ was added to a solution of propionaldehyde $(1.44 \mathrm{~mL}$, $20 \mathrm{mmol})$ and DBU $(4.77 \mathrm{~mL}, 32 \mathrm{mmol})$ in dichloromethane $(28 \mathrm{~mL})$. The mixture was stirred at room temperature for $6 \mathrm{~h}$. The reaction solution was diluted with brine $(30 \mathrm{~mL})$ and extracted with $\mathrm{CH}_{2} \mathrm{Cl}_{2}(20$ $\mathrm{mL} \times 3$ ). Combined extracts were dried over anhydrous $\mathrm{Na}_{2} \mathrm{SO}_{4}$, and concentrated under reduced pressure. The residue was purified by vacuum distillation to give the tert-butyldimethyl(prop-1-en-1-yloxy)silane 5. The ratio of trans/cis was determined to be $1.2: 1$ by ${ }^{1} \mathrm{H}$ NMR.

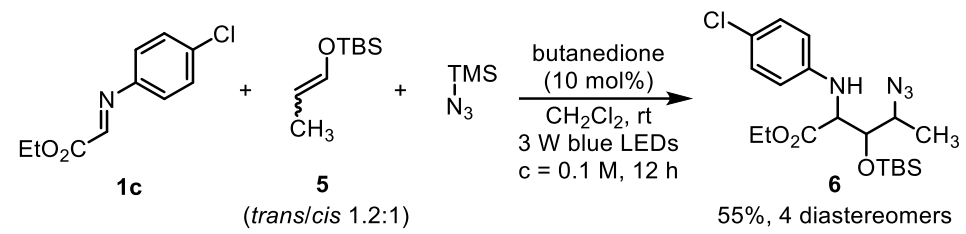

To a test tube charged with a stir bar were added a ethyl-2-((4-chlorophenyl)imino)acetate $1 \mathrm{c}$ (42 mg, 0.2 mmol), tert-butyldimethyl(prop-1-en-1-yloxy)silane 5 (172 $\mathrm{mg}, 1.0 \mathrm{mmol})$. After the test tube was evacuated and backfilled with $\mathrm{N}_{2}$ three times, the solvent $\mathrm{CH}_{2} \mathrm{Cl}_{2}(2 \mathrm{~mL})$ was added through a syringe, followed by the addition of 2,3-butanedione $(1.7 \mu \mathrm{L}, 0.02 \mathrm{mmol}$, note: fluorenone is also able to promote reaction, but it's inseperable with the products) and azidotrimethylsilane ( $32 \mu \mathrm{L}, 0.24 \mathrm{mmol})$. The reaction mixture was stirred under the irradiation of $3 \mathrm{~W}$ blue LEDs at room temperature for $12 \mathrm{~h}$. The progress of the reaction was monitored by TLC. Upon the consumption of imine 1c, the mixture was concentrated in vacuo. The residue was then purified through a silica gel flash column to afford the corresponding product 6. 
Ethyl 4-azido-3-((tert-butyldimethylsilyl)oxy)-2-((4-chlorophenyl)amino)pentanoate (6): compound 6 was isolated through a silica gel flash column $(\mathrm{PE}:$ EtOAc $=100: 1)$ as yellow oil (47 mg, 55\% yield).

Isomer $1+$ Isomer $2(d r=2.3):{ }^{1} \mathrm{H}$ NMR $(600 \mathrm{MHz}$, Chloroform- $d) \delta 7.16-7.09(\mathrm{~m}, 2 \mathrm{H}$, major $+2 \mathrm{H}$, minor), $6.63-6.57$ (m, 2H, major), $6.57-6.52$ (m, 2H, minor), 4.52 (d, J = 7.5 Hz, 1H, major), 4.38 (d, $J=10.7 \mathrm{~Hz}, 1 \mathrm{H}$, minor), 4.34 (d, $J=7.4 \mathrm{~Hz}, 1 \mathrm{H}$, major), $4.30-4.19$ (m, 2H, major $+1 \mathrm{H}$, minor), $4.19-$ 4.10 (m, 2H, minor), $4.02-3.97$ (m, 1H, minor), $3.96-3.87$ (m, 1H, major), $3.74-3.66$ (m, 1H, major $+1 \mathrm{H}$ minor), $1.35-1.31(\mathrm{~m}, 3 \mathrm{H}$, major $+3 \mathrm{H}$, minor), $1.30(\mathrm{t}, J=6.7 \mathrm{~Hz}, 3 \mathrm{H}$, major), $1.22(\mathrm{t}, J=7.3 \mathrm{~Hz}$, $3 \mathrm{H}$, minor), 0.92 (s, 9H, minor), 0.89 (s, 9H, major), 0.12 (s, 3H, minor), 0.08 (s, 3H, major), 0.04 (s, 3H, major), -0.01 (s, 3H, minor); ${ }^{13} \mathrm{C}\left\{{ }^{1} \mathrm{H}\right\} \mathrm{NMR}$ (151 MHz, Chloroform- $d$ ) $\delta 172.1$ (minor), 170.5 (major), 145.8 (minor), 144.6 (major), 129.3 (major), 129.1 (minor), 123.0 (minor), 122.9 (major), 114.9 (minor), 114.5 (major), 76.5 (major), 75.7 (minor), 61.6 (major), 61.5 (minor), 59.3 (major), 59.1 (major), 59.1 (minor), 58.4 (minor), 25.9 (minor), 25.8 (major), 18.2 (minor), 18.1 (major), 16.2 (major), 15.9 (minor), 14.3 (major), 14.1 (minor), -4.0 (major), -4.0 (minor), -4.5 (major), -4.7 (minor) ; IR (neat): 3420, 2955, 2925, 2852, 2097, 1738, 1601, 1500, 1258, $1200 \mathrm{~cm}^{-1}$; HRMS (ESI, m/z): calcd for $\mathrm{C}_{19} \mathrm{H}_{31} \mathrm{ClN}_{4} \mathrm{O}_{3} \mathrm{SiNa}$ $[\mathrm{M}+\mathrm{Na}]^{+} 449.1746$, found 449.1736 .

Isomer 3: ${ }^{1} \mathrm{H}$ NMR $(600 \mathrm{MHz}$, Chloroform- $d$ ) $\delta 7.15-7.09(\mathrm{~m}, 2 \mathrm{H}), 6.55-6.48(\mathrm{~m}, 2 \mathrm{H}), 4.42(\mathrm{~d}, J=$ $10.3 \mathrm{~Hz}, 1 \mathrm{H}), 4.15(\mathrm{q}, J=7.1 \mathrm{~Hz}, 2 \mathrm{H}), 4.08-4.01(\mathrm{~m}, 2 \mathrm{H}), 3.67-3.58(\mathrm{~m}, 1 \mathrm{H}), 1.30(\mathrm{~d}, J=6.7 \mathrm{~Hz}, 3 \mathrm{H})$, $1.22(\mathrm{t}, J=7.1 \mathrm{~Hz}, 3 \mathrm{H}), 0.92(\mathrm{~s}, 9 \mathrm{H}), 0.17$ (s, 3H), 0.04 (s, 3H); ${ }^{13} \mathrm{C}\left\{{ }^{1} \mathrm{H}\right\} \mathrm{NMR}$ (151 MHz, Chloroform-d) $\delta 171.8,145.4,129.2,123.1,114.7,75.6,61.6,60.6,58.0,25.8,18.2,15.8,14.1,-4.4,-5.0$; IR (neat): 3389, 2924, 2853, 2093, 1730, 1602, 1498, 1255, $1192 \mathrm{~cm}^{-1}$; HRMS (ESI, m/z): calcd for $\mathrm{C}_{19} \mathrm{H}_{31} \mathrm{ClN}_{4} \mathrm{O}_{3} \mathrm{SiNa}[\mathrm{M}+\mathrm{Na}]^{+} 449.1746$, found 449.1755 .

Isomer 4: ${ }^{1} \mathrm{H}$ NMR (600 MHz, Chloroform- $d$ ) $\delta 7.15-7.10(\mathrm{~m}, 2 \mathrm{H}), 6.57-6.50(\mathrm{~m}, 2 \mathrm{H}), 4.57(\mathrm{~d}, J=8.6$ $\mathrm{Hz}, 1 \mathrm{H}), 4.20(\mathrm{qd}, J=7.2,2.3 \mathrm{~Hz}, 2 \mathrm{H}), 4.15(\mathrm{dt}, J=6.8,2.8 \mathrm{~Hz}, 1 \mathrm{H}), 3.86-3.81(\mathrm{~m}, 1 \mathrm{H}), 3.63(\mathrm{tt}, J=$ 6.8, 3.8 Hz, 1H), 1.42 (d, J=6.3 Hz, 3H), 1.23 (t, J=7.2 Hz, 3H), $0.91(\mathrm{~s}, 9 \mathrm{H}), 0.12$ (s, 3H), 0.05 (s, 3H);

${ }^{13} \mathrm{C}\left\{{ }^{1} \mathrm{H}\right\}$ NMR (151 MHz, Chloroform- $d$ ) $\delta 171.0,144.9,129.2,123.1,114.6,76.3,61.6,60.0,59.6,25.8$, 18.2, 16.2, 14.2, -4.4, -4.6; IR (neat): 3410, 2925, 2853, 2108, 1737, 1601, 1500, 1255, $1178 \mathrm{~cm}^{-1}$; HRMS (ESI, m/z): calcd for $\mathrm{C}_{19} \mathrm{H}_{31} \mathrm{ClN}_{4} \mathrm{O}_{3} \mathrm{SiNa}[\mathrm{M}+\mathrm{Na}]^{+}$449.1746, found 449.1735.

\section{Mechanistic-Related Experiments}

\section{i. The reaction in the presence/absence of fluorenone}

To a test tube equipped with a stir bar were added imine 1a (39 mg, $0.2 \mathrm{mmol})$ and 9-fluorenone (3.6 mg, $0.02 \mathrm{mmol}$ or none). After the test tube was evacuated and backfilled with $\mathrm{N}_{2}$ three times, acetone $(1 \mathrm{~mL})$ was added through a syringe, followed by the addition of 2,3-dihydrofuran (46 $\mu \mathrm{L}, 0.6 \mathrm{mmol})$ and azidotrimethylsilane ( $32 \mu \mathrm{L}, 0.24 \mathrm{mmol})$, and 1-fluoro-4-iodobenzene ( $23 \mu \mathrm{L}, 0.2 \mathrm{mmol}$, as an internal 
standard). The reaction mixture was stirred under the irradiation of $3 \mathrm{~W}$ blue LEDs at room temperature and $50 \mu \mathrm{L}$ of reaction mixture was taken by a syringe at indicated time $(1.0 \mathrm{~h}, 3.0 \mathrm{~h}, 6.0 \mathrm{~h}, 24 \mathrm{~h})$. The mixture was diluted with chloroform- $d(0.5 \mathrm{~mL})$ and submitted for quantitative ${ }^{19} \mathrm{~F}$ NMR analysis. The results are list in Table $\mathbf{S 2}$.

Table S2. The reaction process in the presence/absence of fluorenone

\begin{tabular}{|c|c|c|}
\hline $\begin{array}{ll}\mathrm{n} / \mathrm{h} & \mathrm{n}_{4 \mathrm{aa}}(\mathrm{mmol})\end{array}$ & Fluorenone $10 \%$ & No fluorenone \\
\hline 0 & 0.000 & 0.000 \\
\hline 1.0 & 0.028 & $<0.010$ \\
\hline 3.0 & 0.076 & 0.030 \\
\hline 6.0 & 0.110 & 0.076 \\
\hline 24.0 & 0.178 & 0.140 \\
\hline
\end{tabular}

\section{ii. Reactions with different concentration of imine 1a}

To a test tube equipped with a stir bar were added imine $1 \mathrm{a}(19.5 \mathrm{mg}, 0.1 \mathrm{mmol}$ or $39 \mathrm{mg}, 0.2 \mathrm{mmol})$ and 9-fluorenone ( $3.6 \mathrm{mg}, 0.02 \mathrm{mmol}$ ). After the test tube was evacuated and backfilled with $\mathrm{N}_{2}$ three times, acetone $(1 \mathrm{~mL})$ was added through a syringe, followed by the addition of 2,3-dihydrofuran (46 $\mu \mathrm{L}, 0.6$ mmol) and azidotrimethylsilane (32 $\mu \mathrm{L}, 0.24 \mathrm{mmol}$ ), and 1-fluoro-4-iodobenzene ( $23 \mu \mathrm{L}, 0.2 \mathrm{mmol}$, as an internal standard). The reaction mixture was stirred under the irradiation of $3 \mathrm{~W}$ blue LEDs at room temperature and $50 \mu \mathrm{L}$ of reaction mixture was taken by a syringe at indicated time $(0.5 \mathrm{~h}, 1.0 \mathrm{~h}, 2.0 \mathrm{~h}$, 3.0 h). The mixture was diluted with chloroform- $d(0.5 \mathrm{~mL})$ and submitted for quantitative ${ }^{19} \mathrm{~F}$ NMR analysis. The results are list in Table S3 and Figure S1.

Table S3. The reaction process with different concentration of imine 1a

\begin{tabular}{|c|c|c|}
\hline $\mathrm{n} / \mathrm{h}$ & $0.1 \mathrm{mmol} \mathbf{1 a}$ & $0.2 \mathrm{mmol} \mathbf{1 a}$ \\
\hline 0 & 0.000 & 0.000 \\
\hline 0.5 & 0.004 & 0.010 \\
\hline 1.0 & 0.012 & 0.028 \\
\hline 2.0 & 0.024 & 0.052 \\
\hline 3.0 & 0.034 & 0.076 \\
\hline
\end{tabular}




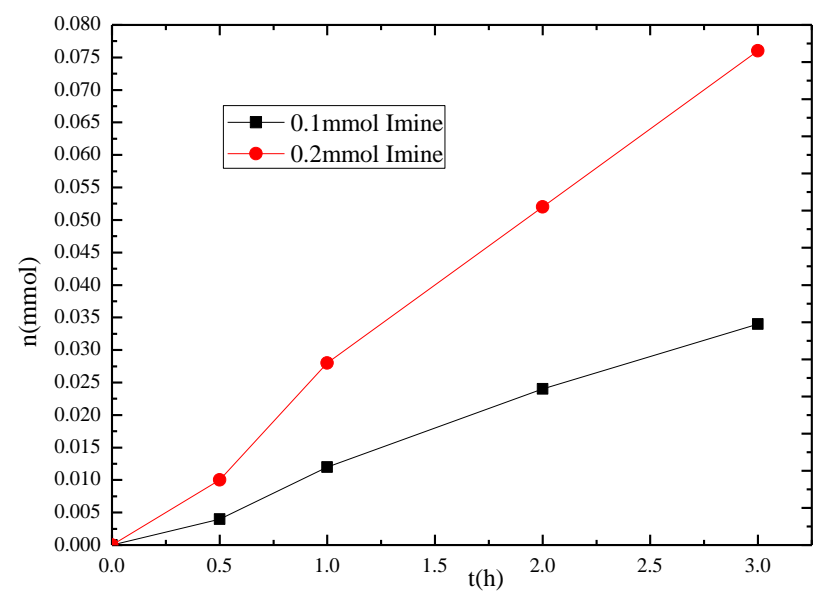

Figure S1. The reaction process with different concentration of imine 1a

\section{iii. Reactions with different concentration of 2,3-dihydrofuran 2a}

To a test tube equipped with a stir bar were added imine $1 \mathbf{a}(39 \mathrm{mg}, 0.2 \mathrm{mmol})$ and 9-fluorenone (3.6 mg, $0.02 \mathrm{mmol})$. After the test tube was evacuated and backfilled with $\mathrm{N}_{2}$ three times, acetone $(1 \mathrm{~mL})$ was added through a syringe, followed by the addition of 2,3-dihydrofuran $(23 \mu \mathrm{L}, 0.3 \mathrm{mmol}$ or $46 \mu \mathrm{L}, 0.6$ $\mathrm{mmol}$ ) and azidotrimethylsilane (32 $\mu \mathrm{L}, 0.24 \mathrm{mmol})$, and 1-fluoro-4-iodobenzene ( $23 \mu \mathrm{L}, 0.2 \mathrm{mmol}$, as an internal standard). The reaction mixture was stirred under the irradiation of $3 \mathrm{~W}$ blue LEDs at room temperature and $50 \mu \mathrm{L}$ of reaction mixture was taken by a syringe at indicated time $(0.5 \mathrm{~h}, 1.0 \mathrm{~h}, 2.0 \mathrm{~h}$, $3.0 \mathrm{~h})$. The mixture was diluted with chloroform- $d(0.5 \mathrm{~mL})$ and submitted for quantitative ${ }^{19} \mathrm{~F}$ NMR analysis. The results are list in Table S4 and Figure S2.

Table S4. The reaction process with different concentration of dihydrofuran $\mathbf{2 a}$

\begin{tabular}{|c|c|c|}
\hline $\mathrm{n}_{\text {4aa }}(\mathrm{mmol})$ & $0.3 \mathrm{mmol} \mathbf{2 a}$ & $0.6 \mathrm{mmol} \mathbf{2 a}$ \\
\hline 0 & 0.000 & 0.000 \\
\hline 0.5 & 0.006 & 0.010 \\
\hline 1.0 & 0.020 & 0.028 \\
\hline 2.0 & 0.048 & 0.052 \\
\hline 3.0 & 0.074 & 0.076 \\
\hline
\end{tabular}




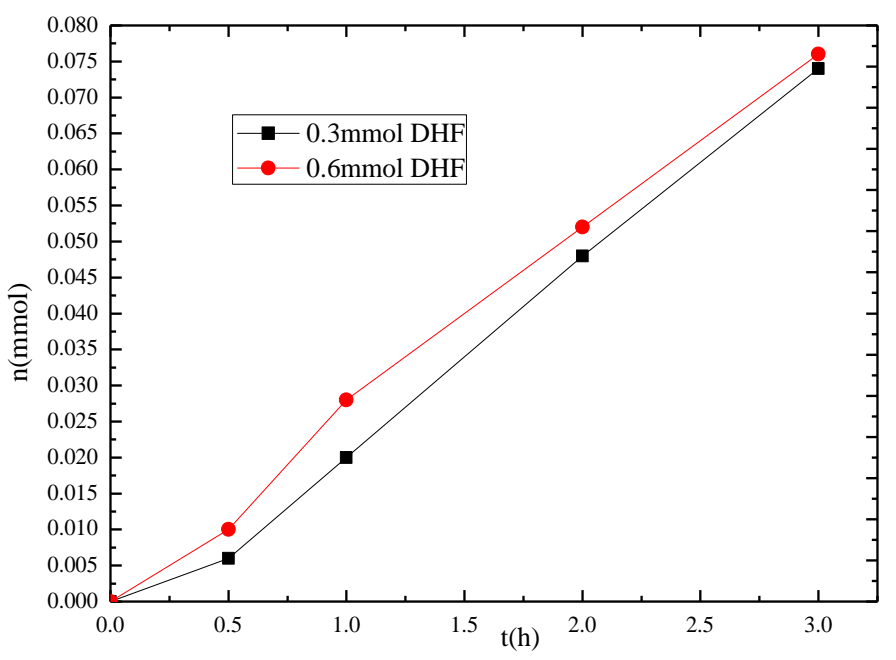

Figure S2. The reaction process with different concentration of imine 2a

iv. The isomerization of enol ether in the presence and in the absence of fluorenone

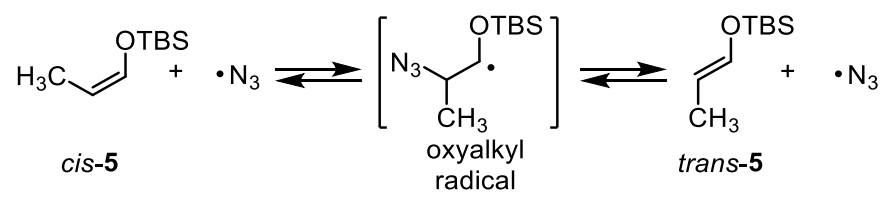

To a test tube equipped with a stir bar was added 9-fluorenone ( $3.6 \mathrm{mg}, 0.02 \mathrm{mmol}$ or none). After the test tube was evacuated and backfilled with $\mathrm{N}_{2}$ three times, acetone $(1 \mathrm{~mL})$ was added through a syringe, followed by the addition of tert-butyldimethyl(prop-1-en-1-yloxy)silane 5 (34 mg, $0.2 \mathrm{mmol}$ ) and azidotrimethylsilane $(32 \mu \mathrm{L}, 0.24 \mathrm{mmol})$, and dodecane $(23 \mu \mathrm{L}, 0.1 \mathrm{mmol})$. The reaction mixture was stirred under the irradiation of $3 \mathrm{~W}$ blue LEDs at room temperature and $50 \mu \mathrm{L}$ of reaction mixture was taken by a syringe at indicated time. The mixture was diluted with $1.5 \mathrm{ml}$ petroleum ether and submitted for gas chromatography analysis. The results are list in Table S5.

Table S5. Isomerization of enol ether in the presence and in the absence of fluorenone

\begin{tabular}{|c|c|c|c|}
\hline Entry & $\mathrm{n}_{E}(\mathrm{mmol})$ & $\mathrm{n}_{z}(\mathrm{mmol})$ & $E / Z$ \\
\hline $\begin{array}{c}\text { In the absence of } \\
\text { fluorenone }(3 \mathrm{~h})\end{array}$ & 0.1259 & 0.0741 & 1.7 \\
\hline $\begin{array}{c}\text { In the presence of } \\
\text { fluorenone }(3 \mathrm{~h})\end{array}$ & 0.1685 & 0.0315 & 5.3 \\
\hline
\end{tabular}

\section{Product Derivatization}

i. The reduction of carboxylate group

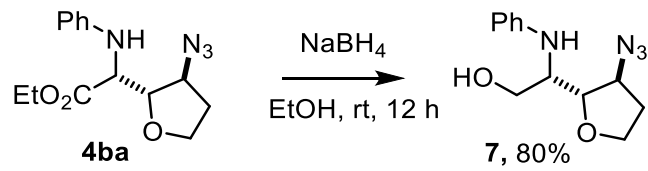


2-(3-Azidotetrahydrofuran-2-yl)-2-(phenylamino)ethan-1-ol (7): $\mathrm{NaBH}_{4}(12 \mathrm{mg}, 0.3 \mathrm{mmol})$ was added to the solution of $\mathbf{4 b a}$ (isomer $1,29 \mathrm{mg}, 0.1 \mathrm{mmol})$ in $\mathrm{EtOH}(1 \mathrm{~mL})$ at $0{ }^{\circ} \mathrm{C}$. The mixture was warmed to room temperature and stirred for 12 hours. After $\mathbf{4 b a}$ was consumed as indicated by TLC, the reaction was quenched with brine and extracted with $\mathrm{CH}_{2} \mathrm{Cl}_{2}(10 \mathrm{~mL} \times 3)$. The combined organic layers were dried over anhydrous $\mathrm{Na}_{2} \mathrm{SO}_{4}$, and concentrated under reduced pressure. The residue was purified through a silica gel flash column $(\mathrm{PE} / \mathrm{EtOAc}=5: 1)$ to give the product 7 as colorless oil $(20 \mathrm{mg}, 80 \%) .{ }^{1} \mathrm{H} \mathrm{NMR}$ $(600 \mathrm{MHz}$, Chloroform- $d$ ) $\delta 7.20(\mathrm{t}, J=7.6 \mathrm{~Hz}, 2 \mathrm{H}), 6.76(\mathrm{t}, J=7.4 \mathrm{~Hz}, 1 \mathrm{H}), 6.67(\mathrm{~d}, J=8.0 \mathrm{~Hz}, 2 \mathrm{H})$, $4.08(\mathrm{dt}, J=7.6,3.8 \mathrm{~Hz}, 1 \mathrm{H}), 4.02(\mathrm{td}, J=8.3,3.8 \mathrm{~Hz}, 1 \mathrm{H}), 3.97(\mathrm{br}, 1 \mathrm{H}), 3.93-3.85(\mathrm{~m}, 1 \mathrm{H}), 3.87-$ $3.79(\mathrm{~m}, 2 \mathrm{H}), 3.76(\mathrm{dt}, J=10.6,4.4 \mathrm{~Hz}, 1 \mathrm{H}), 3.44(\mathrm{~d}, J=7.3 \mathrm{~Hz}, 1 \mathrm{H}), 2.51(\mathrm{t}, J=5.8 \mathrm{~Hz}, 1 \mathrm{H}), 2.28-$ $2.18(\mathrm{~m}, 1 \mathrm{H}), 2.01(\mathrm{ddt}, J=13.7,7.1,3.7 \mathrm{~Hz}, 1 \mathrm{H}) ;{ }^{13} \mathrm{C}\left\{{ }^{1} \mathrm{H}\right\} \mathrm{NMR}(151 \mathrm{MHz}$, Chloroform- $d) \delta 146.7,129.5$, 118.4, 113.8, 85.1, 67.4, 63.6, 62.2, 56.1, 32.0; IR (neat): 3361, 2943, 2877, 2097, 1601, 1497, 1436, 1317, 1256, 1180, 1065, $\mathrm{cm}^{-1}$; HRMS (ESI, m/z): calcd for $\mathrm{C}_{12} \mathrm{H}_{16} \mathrm{~N}_{4} \mathrm{O}_{2} \mathrm{Na}[\mathrm{M}+\mathrm{Na}]^{+} 271.1165$, found 271.1176.

\section{ii. The hydrolysis of carboxylate group}

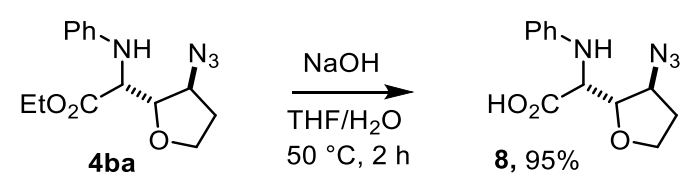

2-(3-Azidotetrahydrofuran-2-yl)-2-(phenylamino)acetic acid (8): To a well stirred solution of compound 4ba (isomer 1, $29 \mathrm{mg}, 0.1 \mathrm{mmol})$ in THF (1 mL), $1 \mathrm{M} \mathrm{NaOH}$ solution $(0.5 \mathrm{~mL})$ was added at room temperature, then the mixture was stirred at $50{ }^{\circ} \mathrm{C}$ for $2 \mathrm{~h}$. THF was removed, and the residue was extracted with water and EtOAc. The aqueous layer was acidified with cold $\mathrm{HCl}$ and extracted with EtOAc $(3 \times 15 \mathrm{~mL})$. The combined organic layers were dried over anhydrous $\mathrm{Na}_{2} \mathrm{SO}_{4}$, and concentrated under reduced pressure. The residue was purified through a silica gel flash column $(\mathrm{PE} /$ Acetone $=1: 1)$ to give the product 8 as white solid (25 mg, 95\% yield, m.p. $\left.123-124{ }^{\circ} \mathrm{C}\right) ;{ }^{1} \mathrm{H}$ NMR $\left(600 \mathrm{MHz}, \mathrm{DMSO}-d_{6}\right)$ $\delta 7.09(\mathrm{t}, J=7.7 \mathrm{~Hz}, 2 \mathrm{H}), 6.70(\mathrm{~d}, J=8.0 \mathrm{~Hz}, 2 \mathrm{H}), 6.61(\mathrm{t}, J=7.3 \mathrm{~Hz}, 1 \mathrm{H}), 5.99(\mathrm{br}, 1 \mathrm{H}), 4.22(\mathrm{dt}, J=$ 6.5, $2.9 \mathrm{~Hz}, 1 \mathrm{H}), 4.05(\mathrm{dd}, J=7.7,2.8 \mathrm{~Hz}, 1 \mathrm{H}), 3.96(\mathrm{~d}, J=7.7 \mathrm{~Hz}, 1 \mathrm{H}), 3.91(\mathrm{td}, J=8.3,4.4 \mathrm{~Hz}, 1 \mathrm{H})$, $3.79(\mathrm{td}, J=8.0 \mathrm{~Hz}, 1 \mathrm{H}), 3.35(\mathrm{br}, 1 \mathrm{H}), 2.32(\mathrm{dq}, J=13.6,7.9 \mathrm{~Hz}, 1 \mathrm{H}), 1.94$ (ddt, $J=13.7,7.3,3.8 \mathrm{~Hz}$, $1 \mathrm{H}) ;{ }^{13} \mathrm{C}\left\{{ }^{1} \mathrm{H}\right\} \mathrm{NMR}\left(151 \mathrm{MHz}, \mathrm{DMSO}-d_{6}\right) \delta 173.3,147.9,129.3,117.5,113.6,84.4,67.2,63.8,58.8,31.9$; IR (neat): 3304, 2989, 2882, 2491, 2099, 1714, 1601, 1498, 1417, 1259, 1198, $1088 \mathrm{~cm}^{-1}$; HRMS (ESI, $\mathrm{m} / \mathrm{z}$ ): calcd for $\mathrm{C}_{12} \mathrm{H}_{14} \mathrm{~N}_{4} \mathrm{O}_{3} \mathrm{Na}[\mathrm{M}+\mathrm{Na}]^{+}$285. 0958, found 285. 0975.

\section{iii. The reduction of azide group}

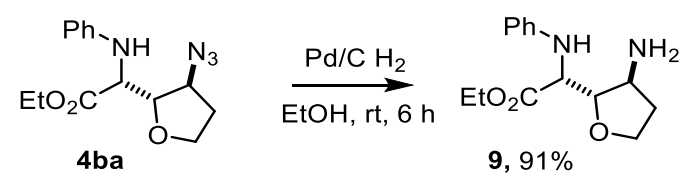


Ethyl-2-(3-aminotetrahydrofuran-2-yl)-2-(phenylamino)acetate (9): 4ba (isomer 1, $29 \mathrm{mg}, 0.1 \mathrm{mmol}$ ) was dissolved in EtOH $(1 \mathrm{~mL})$ and transfered to a $10 \mathrm{~mL}$ sealable tube charged with a stir bar and palladium on charcoal ( $3 \mathrm{mg}, 10 \mathrm{wt}$. \%) under $\mathrm{N}_{2}$. The tube was then evacuated and backfilled with $\mathrm{H}_{2}$ three times with a $\mathrm{H}_{2}$ balloon. The reaction mixture was stirred vigorously under $\mathrm{H}_{2}$ atmosphere at room temperature for $6 \mathrm{~h}$. The mixture was filtered through a Celite ${ }^{\circledR}$ pad and concentrated in vacuo. The residue was purified through a silica gel flash column $\left(\mathrm{CH}_{2} \mathrm{Cl}_{2} / \mathrm{CH}_{3} \mathrm{OH}=10: 1\right)$ to give the product 9 as colorless oil (24 mg, 91\%). ${ }^{1} \mathrm{H}$ NMR (600 MHz, Chloroform- $d$ ) $\delta 7.20-7.12(\mathrm{~m}, 2 \mathrm{H}), 6.75(\mathrm{t}, J=7.4 \mathrm{~Hz}, 1 \mathrm{H})$, $6.70(\mathrm{~d}, J=7.8 \mathrm{~Hz}, 2 \mathrm{H}), 4.54(\mathrm{~d}, J=8.9 \mathrm{~Hz}, 1 \mathrm{H}), 4.20(\mathrm{q}, J=7.1 \mathrm{~Hz}, 2 \mathrm{H}), 4.13(\mathrm{dd}, J=8.3,5.9 \mathrm{~Hz}, 1 \mathrm{H})$, $3.98-3.88(\mathrm{~m}, 2 \mathrm{H}), 3.77(\mathrm{dd}, J=5.8 \mathrm{~Hz}, 1 \mathrm{H}), 3.57(\mathrm{dt}, J=6.4 \mathrm{~Hz}, 1 \mathrm{H}), 2.21(\mathrm{dq}, J=13.7,7.1 \mathrm{~Hz}, 1 \mathrm{H})$, $1.75-1.66(\mathrm{~m}, 1 \mathrm{H}), 1.41(\mathrm{br}, 2 \mathrm{H}), 1.24(\mathrm{t}, J=7.1 \mathrm{~Hz}, 3 \mathrm{H}) ;{ }^{13} \mathrm{C}\left\{{ }^{1} \mathrm{H}\right\} \mathrm{NMR}(151 \mathrm{MHz}$, Chloroform- $d$ ) $\delta$ 172.0, 146.8, 129.3, 118.7, 114.0, 86.8, 67.3, 61.3, 60.0, 54.8, 36.1, 14.2; IR (neat): 3355, 2978, 1729, 1602, 1498, 1313, 1260, $1179 \mathrm{~cm}^{-1}$; HRMS (ESI, m/z): calcd for $\mathrm{C}_{14} \mathrm{H}_{20} \mathrm{~N}_{2} \mathrm{O}_{3} \mathrm{Na}[\mathrm{M}+\mathrm{Na}]^{+} 287.1366$, found 287.1364 .

\section{iv. The copper(I)-catalyzed azide-alkyne cycloaddition}

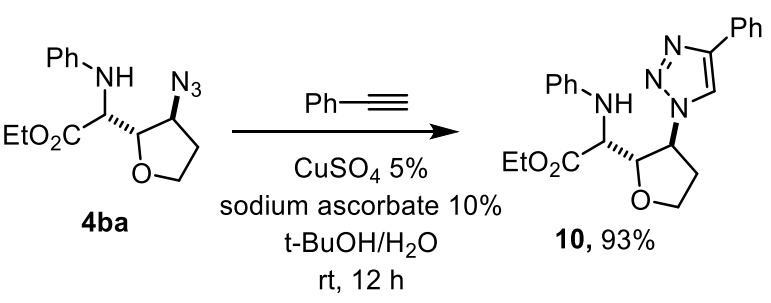

Ethyl-2-(3-(4-phenyl-1H-1,2,3-triazol-1-yl)tetrahydrofuran-2-yl)-2-(phenylamino)acetate (10): To a solution of compound $4 \mathbf{b a}$ (isomer 1, $58 \mathrm{mg}, 0.2 \mathrm{mmol}$ ) in $t-\mathrm{BuOH}: \mathrm{H}_{2} \mathrm{O}(1: 1,1 \mathrm{~mL}$ ) at room temperature was added $\mathrm{CuSO}_{4}(1.6 \mathrm{mg}, 0.01 \mathrm{mmol})$ and sodium ascorbate $(4 \mathrm{mg}, 0.02 \mathrm{mmol})$ and ethynylbenzene ( $24.5 \mathrm{mg}, 0.24 \mathrm{mmol})$. The reaction mixture was stirred for $12 \mathrm{~h}$ at room temperature. After $4 \mathbf{b a}$ was consumed as indicated by TLC, the reaction was quenched with brine and extracted with EtOAc $(10 \mathrm{~mL}$ $\times 3)$. The residue was purified through a silica gel flash column $(\mathrm{PE} /$ Acetone $=5: 1)$ to give the product 10 as white solid (73 mg, 93\% yield, m.p. $\left.105-106{ }^{\circ} \mathrm{C}\right) ;{ }^{1} \mathrm{H}$ NMR $(600 \mathrm{MHz}$, Chloroform- $d$ ) $\delta 7.82(\mathrm{dd}$, $J=8.2,1.4 \mathrm{~Hz}, 2 \mathrm{H}), 7.79(\mathrm{~s}, 1 \mathrm{H}), 7.43(\mathrm{t}, J=7.6 \mathrm{~Hz}, 2 \mathrm{H}), 7.37-7.31(\mathrm{~m}, 1 \mathrm{H}), 7.21-7.16(\mathrm{~m}, 2 \mathrm{H}), 6.80$ $(\mathrm{tt}, J=7.3,1.1 \mathrm{~Hz}, 1 \mathrm{H}), 6.77-6.72(\mathrm{~m}, 2 \mathrm{H}), 5.34-5.27(\mathrm{~m}, 1 \mathrm{H}), 4.64-4.56(\mathrm{~m}, 2 \mathrm{H}), 4.32(\mathrm{t}, J=5.4$ $\mathrm{Hz}, 1 \mathrm{H}), 4.26-4.20(\mathrm{~m}, 1 \mathrm{H}), 4.19-4.12(\mathrm{~m}, 2 \mathrm{H}), 4.13-4.04(\mathrm{~m}, 1 \mathrm{H}), 2.59$ (dq, $J=13.8,8.0 \mathrm{~Hz}, 1 \mathrm{H})$, $2.52(\mathrm{ddt}, J=13.5,7.5,4.9 \mathrm{~Hz}, 1 \mathrm{H}), 1.16(\mathrm{t}, J=7.1 \mathrm{~Hz}, 3 \mathrm{H}) ;{ }^{13} \mathrm{C}\left\{{ }^{1} \mathrm{H}\right\} \mathrm{NMR}$ (101 MHz, Chloroform- $d$ ) $\delta$ 171.1, 147.9, 146.6, 130.4, 129.4, 128.9, 128.3, 125.7, 119.2, 119.0, 114.2, 85.1, 68.2, 62.0, 61.9, 59.4, 33.4, 14.1; IR (neat): 3355, 2981, 1729, 1602, 1498, 1483, 1462, 1306, 1259, 1181, $1076 \mathrm{~cm}^{-1}$; HRMS (ESI, m/z): calcd for $\mathrm{C}_{22} \mathrm{H}_{24} \mathrm{~N}_{4} \mathrm{O}_{3} \mathrm{Na}[\mathrm{M}+\mathrm{Na}]^{+}$415.1741, found 415.1726. 


\section{v. The synthesis of morpholines}
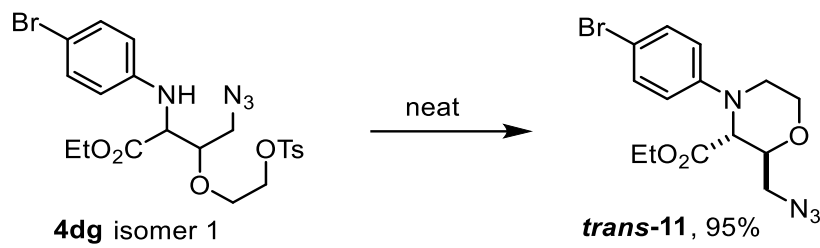

trans-Ethyl-2-(azidomethyl)-4-(4-bromophenyl)morpholine-3-carboxylate (trans-11): 4dg ( isomer 1, less polar) (54 mg, $0.1 \mathrm{mmol}$ ) was stored at room temperature for 24 hours without solvent. The residue was purified through a silica gel flash column $(\mathrm{PE} / \mathrm{EtOAc}=10: 1)$ to give the product trans-11 as colourless oil (35 mg, 95\%); ${ }^{1} \mathrm{H}$ NMR (600 MHz, Chloroform- $d$ ) $\delta 7.41-7.32(\mathrm{~m}, 2 \mathrm{H}), 6.84-6.78(\mathrm{~m}$, 2H), $4.26(\mathrm{td}, J=6.2,4.1 \mathrm{~Hz}, 1 \mathrm{H}), 4.13(\mathrm{q}, J=7.1 \mathrm{~Hz}, 2 \mathrm{H}), 4.05(\mathrm{~d}, J=4.1 \mathrm{~Hz}, 1 \mathrm{H}), 3.96$ (ddd, $J=11.7$, 8.0, 3.6 Hz, 1H), 3.87 (ddd, $J=11.7,5.3,3.7 \mathrm{~Hz}, 1 \mathrm{H}), 3.69$ (dd, $J=12.7,6.5 \mathrm{~Hz}, 1 \mathrm{H}), 3.60$ (dd, $J=12.7$, $5.9 \mathrm{~Hz}, 1 \mathrm{H}), 3.47$ (ddd, $J=11.8,8.0,3.7 \mathrm{~Hz}, 1 \mathrm{H}), 3.13$ (dt, $J=12.1,4.5 \mathrm{~Hz}, 1 \mathrm{H}), 1.17$ (t, $J=7.1 \mathrm{~Hz}, 3 \mathrm{H}$ ); ${ }^{13} \mathrm{C}$ NMR $\left(151 \mathrm{MHz}, \mathrm{CDCl}_{3}\right) \delta 169.9,149.5,132.1,119.2,113.9,74.0,62.6,61.5,60.7,50.6,46.8,14.1 ;$ IR (neat): 2978, 2872, 2098, 1738, 1589, 1494, 1297, 1176, 1124, $1076 \mathrm{~cm}^{-1}$; HRMS (ESI, m/z): calcd for $\mathrm{C}_{14} \mathrm{H}_{17} \mathrm{BrN}_{4} \mathrm{O}_{3} \mathrm{Na}[\mathrm{M}+\mathrm{Na}]^{+}$391.0376, found 391.0366. The relative stereochemistry of trans-11 was determined through NOE analysis.

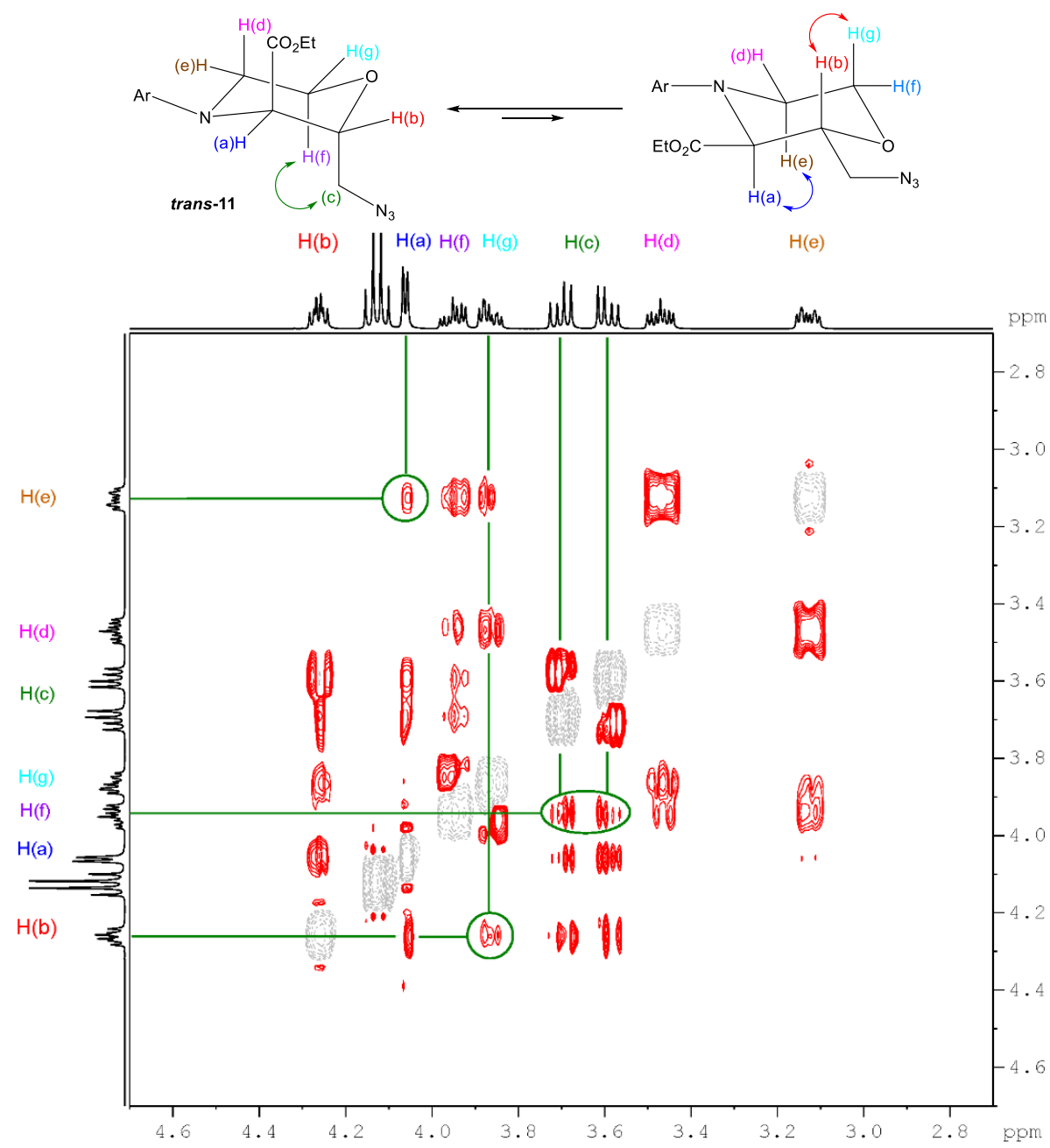



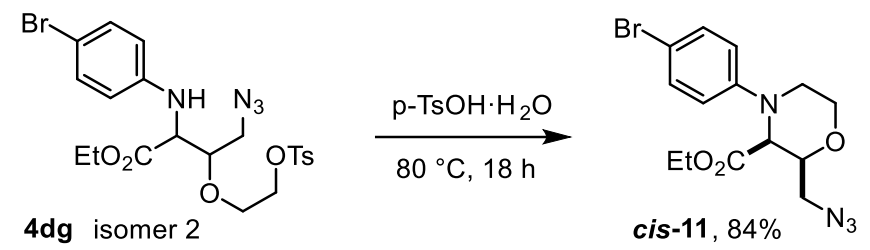

cis-Ethyl-2-(azidomethyl)-4-(4-bromophenyl)morpholine-3-carboxylate (cis-11): 4dg ( isomer 2, more polar) $(54 \mathrm{mg}, 0.1 \mathrm{mmol})$ was dissolved in $\mathrm{EtOH}(0.5 \mathrm{~mL})$. Add $p$-Toluenesulfonic acid monohydrate $(0.02 \mathrm{mmol})$ to the mixture, which was stirred for $18 \mathrm{~h}$ at $80{ }^{\circ} \mathrm{C}$. The mixture was concentrated in vacuo. The residue was purified through a silica gel flash column $(\mathrm{PE} / \mathrm{EtOAc}=10: 1)$ to give the product cis-11 as colourless oil (31 mg, 84\%); ${ }^{1} \mathrm{H}$ NMR (400 MHz, Chloroform- $d$ ) $\delta 7.38-7.31$ (m, 2H), $6.77-6.69(\mathrm{~m}, 2 \mathrm{H}), 4.30(\mathrm{~d}, J=3.5 \mathrm{~Hz}, 1 \mathrm{H}), 4.20(\mathrm{ddd}, J=7.2,3.9,1.2 \mathrm{~Hz}, 1 \mathrm{H}), 4.12(\mathrm{qd}, J=$ 7.1, 2.2 Hz, 2H), $3.98(\mathrm{ddd}, J=7.3,5.1,3.6 \mathrm{~Hz}, 1 \mathrm{H}), 3.82(\mathrm{ddd}, J=12.3,11.1,3.2 \mathrm{~Hz}, 1 \mathrm{H}), 3.71(\mathrm{td}, J=$ 11.9, $3.9 \mathrm{~Hz}, 1 \mathrm{H}), 3.58-3.43(\mathrm{~m}, 2 \mathrm{H}), 3.23(\mathrm{dd}, J=11.3,2.8 \mathrm{~Hz}, 1 \mathrm{H}), 1.17(\mathrm{t}, J=7.1 \mathrm{~Hz}, 3 \mathrm{H}) ;{ }^{13} \mathrm{C} \mathrm{NMR}$ $\left(101 \mathrm{MHz}, \mathrm{CDCl}_{3}\right) \delta 169.5,148.5,132.1,116.4,112.2,76.7,67.2,61.2,59.4,52.3,43.0,14.2$; IR (neat): 2922, 2864, 2097, 1738, 1588, 1493, 1291, 1172, 1116, $1080 \mathrm{~cm}^{-1} ;$ HRMS (ESI, m/z): calcd for $\mathrm{C}_{14} \mathrm{H}_{17} \mathrm{BrN}_{4} \mathrm{O}_{3} \mathrm{Na}[\mathrm{M}+\mathrm{Na}]^{+}$391.0376, found 391.0364. The relative stereochemistry of cis-11 was determined through NOE analysis.

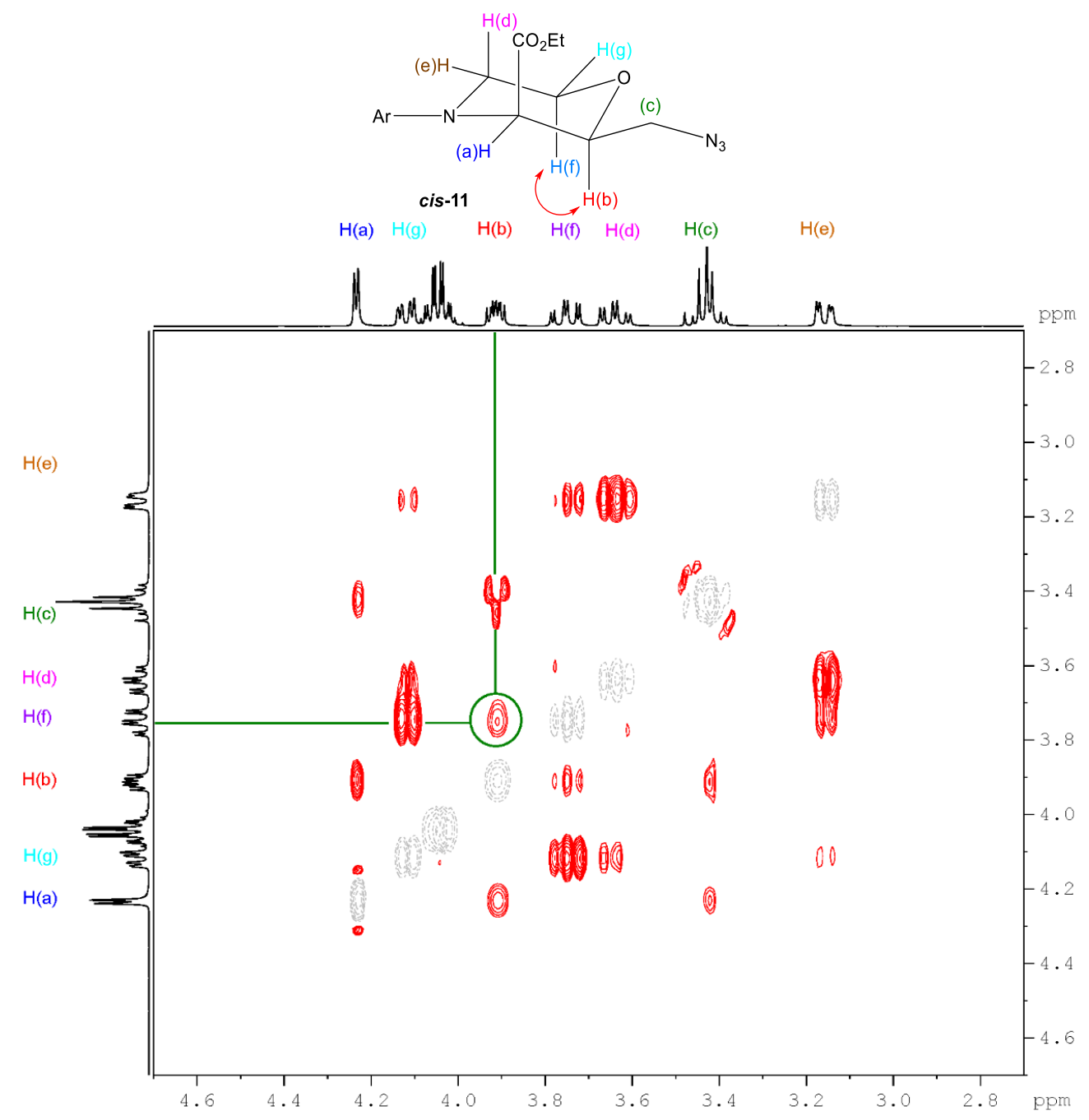




\section{vi. The Removal of the PMP group}

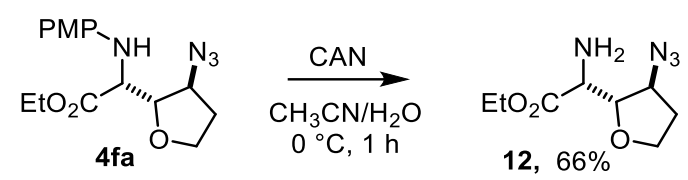

Ethyl-2-amino-2-(3-azidotetrahydrofuran-2-yl)acetate (12): To a solution of CAN (ceric ammonium nitrate, $219 \mathrm{mg}, 0.4 \mathrm{mmol}$ ) in $0.7 \mathrm{ml} \mathrm{H} \mathrm{H}_{2} \mathrm{O}$ at $0{ }^{\circ} \mathrm{C}$ was added $4 \mathbf{f a}$ (isomer $1,32 \mathrm{mg}, 0.1 \mathrm{mmol}$ ) in $0.3 \mathrm{ml}$ $\mathrm{CH}_{3} \mathrm{CN}$. The reaction mixture was stirred for $1 \mathrm{~h}$ at $0{ }^{\circ} \mathrm{C}$. The mixture was modulated to alkalescence with saturated aqueous sodium carbonate. Then the mixture was extracted by DCM for three times, washed with brine, dried over $\mathrm{Na}_{2} \mathrm{SO}_{4}$ and concentrated in vacuo. The residue was purified through a silica gel flash column $(\mathrm{PE} / \mathrm{EtOAc}=1: 1)$ to give the product 12 as yellow oil $(14 \mathrm{mg}, 66 \%) ;{ }^{1} \mathrm{H} \mathrm{NMR}$ $(600 \mathrm{MHz}$, Chloroform- $d) \delta 4.26(\mathrm{dt}, J=7.6,4.7 \mathrm{~Hz}, 1 \mathrm{H}), 4.22(\mathrm{qd}, J=7.1,4.4 \mathrm{~Hz}, 2 \mathrm{H}), 4.04(\mathrm{dd}, J=$ 5.0, $3.2 \mathrm{~Hz}, 1 \mathrm{H}), 3.99$ (ddd, $J=8.6,7.5,4.6 \mathrm{~Hz}, 1 \mathrm{H}), 3.86(\mathrm{td}, J=8.4,6.9 \mathrm{~Hz}, 1 \mathrm{H}), 3.48(\mathrm{~d}, J=3.2 \mathrm{~Hz}$, $1 \mathrm{H}), 2.26(\mathrm{dq}, J=12.9,7.8 \mathrm{~Hz}, 1 \mathrm{H}), 2.01(\mathrm{ddt}, J=12.9,6.9,4.6 \mathrm{~Hz}, 1 \mathrm{H}), 1.62(\mathrm{br}, 2 \mathrm{H}), 1.29(\mathrm{t}, J=7.1$ $\mathrm{Hz}, 3 \mathrm{H}) ;{ }^{13} \mathrm{C}\left\{{ }^{1} \mathrm{H}\right\} \mathrm{NMR}(101 \mathrm{MHz}$, Chloroform- $d$ ) $\delta 173.7,84.4,67.9,62.5,61.5,56.0,32.5,14.2$; IR (neat): 3383, 2983, 2930, 2106, 1739, 1685, 1515, 1372, 1262, 1197, 1072, $1026 \mathrm{~cm}^{-1}$; HRMS (ESI, m/z): calcd for $\mathrm{C}_{8} \mathrm{H}_{14} \mathrm{~N}_{4} \mathrm{O}_{3} \mathrm{Na}[\mathrm{M}+\mathrm{Na}]^{+} 237.0958$, found 237.0963 .

\section{vii. The synthesis of $\gamma$-Lactam}
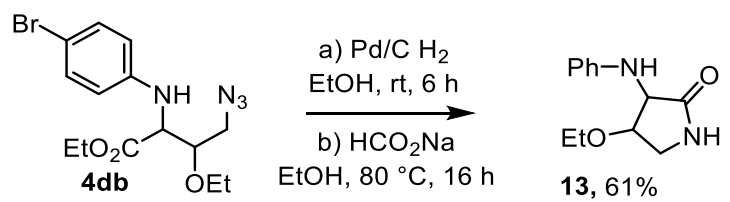

4-Ethoxy-3-(phenylamino)pyrrolidin-2-one (13): 4db (isomer 1, $74 \mathrm{mg}, 0.2 \mathrm{mmol}$ ) was dissolved in EtOH $(2 \mathrm{~mL})$ and transfered to a $10 \mathrm{~mL}$ sealable tube charged with a stir bar and palladium on charcoal (7.4 mg, 10 wt. \%) under $\mathrm{N}_{2}$. The tube was then evacuated and backfilled with $\mathrm{H}_{2}$ three times with a $\mathrm{H}_{2}$ balloon. The reaction mixture was stirred vigorously under $\mathrm{H}_{2}$ atmosphere at room temperature for $6 \mathrm{~h}$. The mixture was filtered through a Celite ${ }^{\circledR}$ pad. The filtrate was directly used for next step without further purification. Add sodium formate $(13.6 \mathrm{mg}, 0.4 \mathrm{mmol})$ to the filtrate and the mixture was stirred for $16 \mathrm{~h}$ at $80{ }^{\circ} \mathrm{C}$. The mixture was acidized by saturated aqueous ammonium chloride. Then the mixture was extracted by EtOAc for three times, washed with brine, dried over $\mathrm{Na}_{2} \mathrm{SO}_{4}$ and concentrated in vacuo. The residue was purified through a silica gel flash column (PE/Acetone $=3: 1)$ to give the product $\mathbf{1 3}$ as red oil (27 mg, 61\%); ${ }^{1} \mathrm{H}$ NMR (400 MHz, Chloroform- $d$ ) $\delta 7.25-7.15(\mathrm{~m}, 2 \mathrm{H}), 6.92-6.84(\mathrm{~m}, 3 \mathrm{H})$, $6.79(\mathrm{tt}, J=7.3,1.1 \mathrm{~Hz}, 1 \mathrm{H}), 4.40(\mathrm{br}, 1 \mathrm{H}), 4.10-3.97(\mathrm{~m}, 2 \mathrm{H}), 3.64(\mathrm{ddd}, J=9.1,7.0,1.8 \mathrm{~Hz}, 1 \mathrm{H}), 3.57$ $(\mathrm{qd}, J=7.0,5.0 \mathrm{~Hz}, 2 \mathrm{H}), 3.31-3.18(\mathrm{~m}, 1 \mathrm{H}), 1.24(\mathrm{t}, J=7.0 \mathrm{~Hz}, 3 \mathrm{H}) ;{ }^{13} \mathrm{C} \mathrm{NMR}\left(101 \mathrm{MHz}, \mathrm{CDCl}_{3}\right) \delta$ 174.3, 147.7, 129.2, 118.6, 114.0, 82.4, 65.8, 61.0, 45.0, 15.4; IR (neat): 3324, 3053, 2974, 2877, 1704 
1602, 1498, 1260, 1121, $1039 \mathrm{~cm}^{-1}$; HRMS (ESI, m/z): calcd for $\mathrm{C}_{12} \mathrm{H}_{16} \mathrm{~N}_{2} \mathrm{O}_{2} \mathrm{Na}[\mathrm{M}+\mathrm{Na}]^{+} 243.1104$, found 243.1111 .

\section{References}

[1] Shi, M.; Ma, G.-N.; Gao, J. J. Org. Chem. 2007, 72, 9779. 


\section{Copies of NMR Spectra for New Compounds}

চ

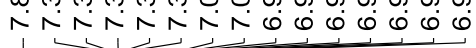

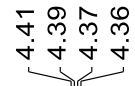

$\mathrm{EtO}_{2} \mathrm{C}$

$1 \mathrm{~g}\left(\mathrm{CDCl}_{3}, 400 \mathrm{MHz}\right)$

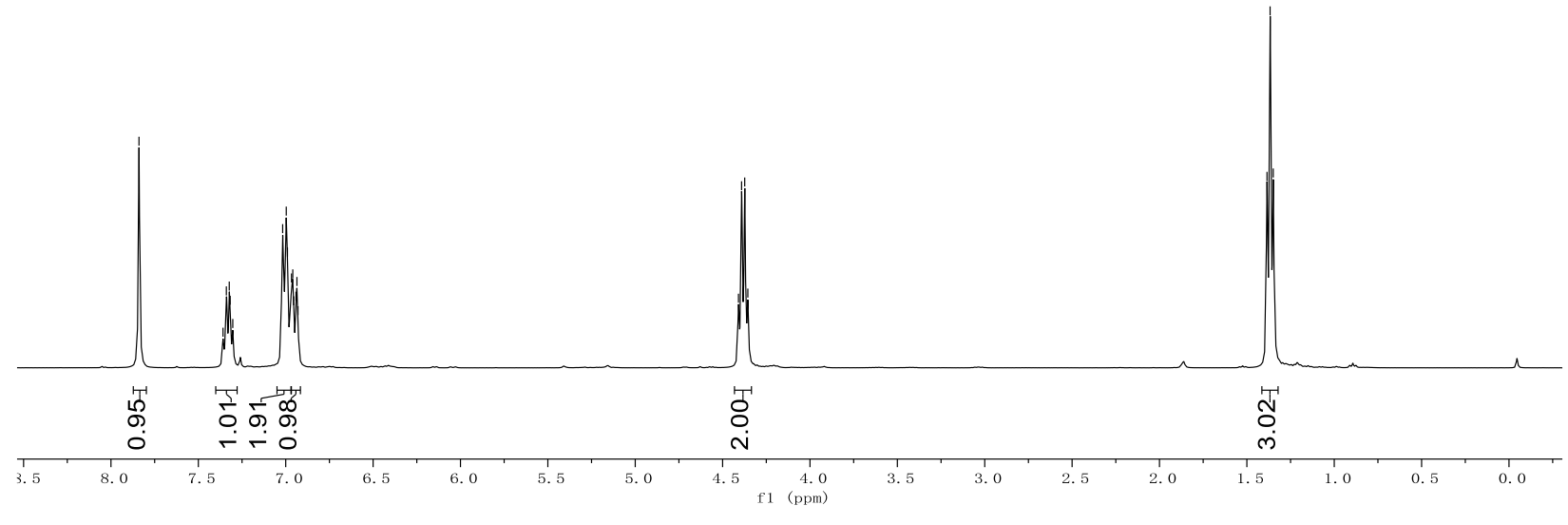

กิ

ษั่

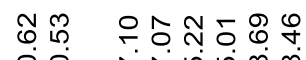

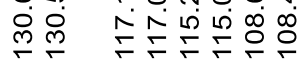

年年宁年

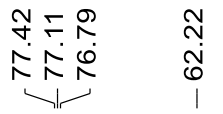

$\stackrel{m}{\stackrel{m}{+}}$<smiles>CC(=O)OCNc1cccc(F)c1</smiles>

$1 \mathrm{~g}\left(\mathrm{CDCl}_{3}, 101 \mathrm{MHz}\right)$
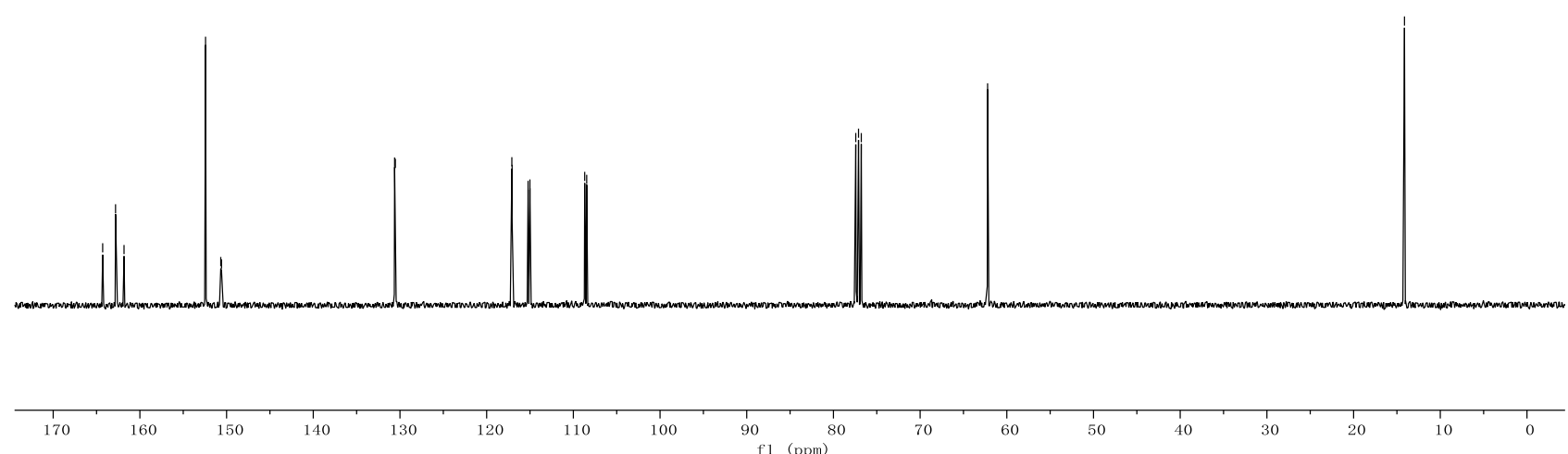
तै

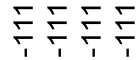

(1,

$1 \mathrm{~g}\left(\mathrm{CDCl}_{3}, 376 \mathrm{MHz}\right)$

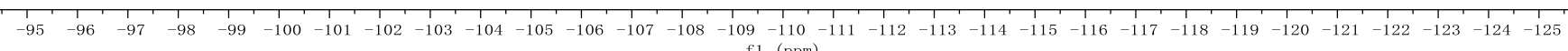




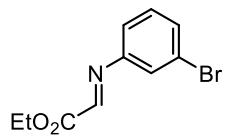

$1 i\left(\mathrm{CDCl}_{3}, 400 \mathrm{MHz}\right)$
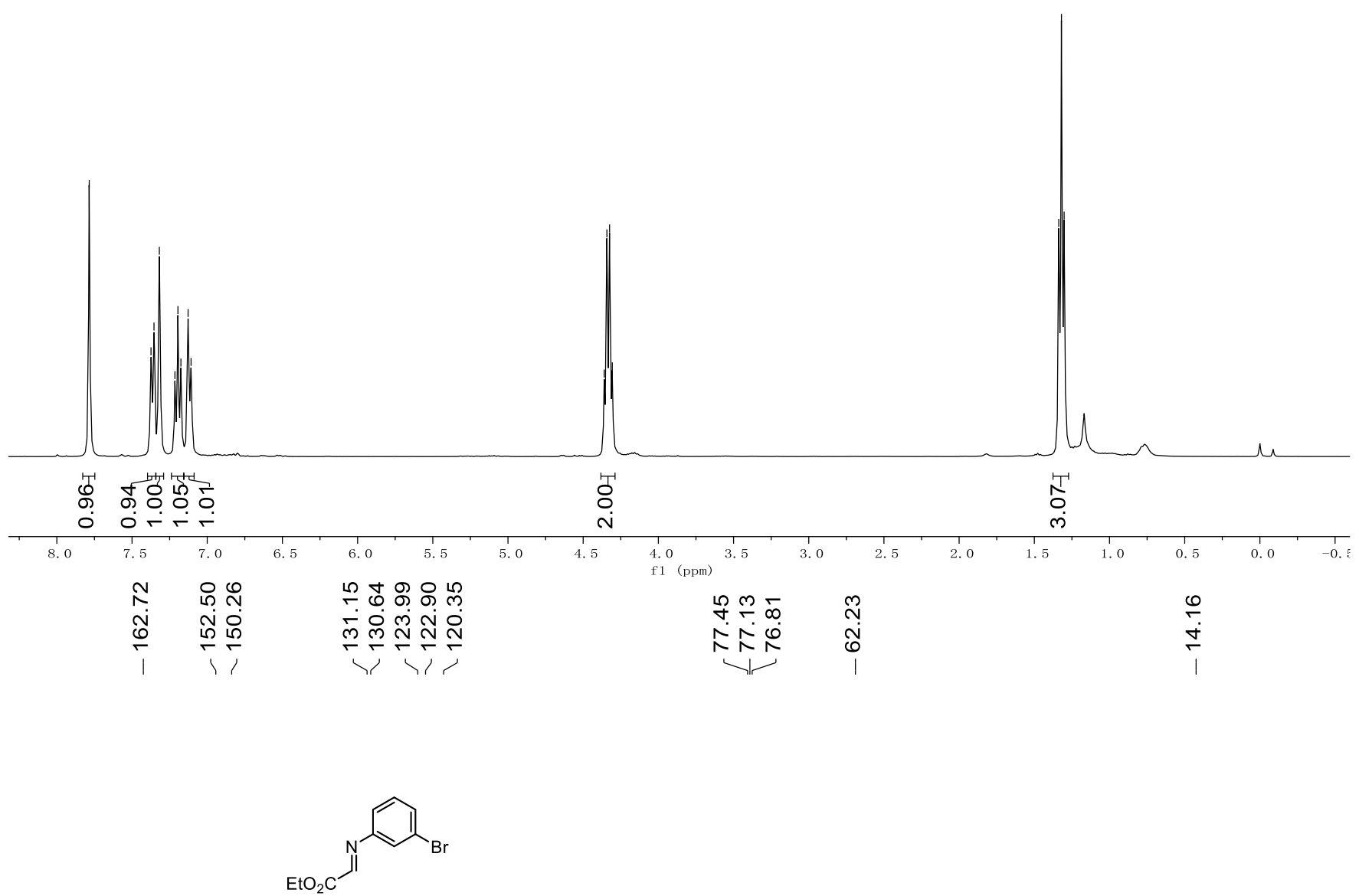

1i $\left(\mathrm{CDCl}_{3}, 101 \mathrm{MHz}\right)$

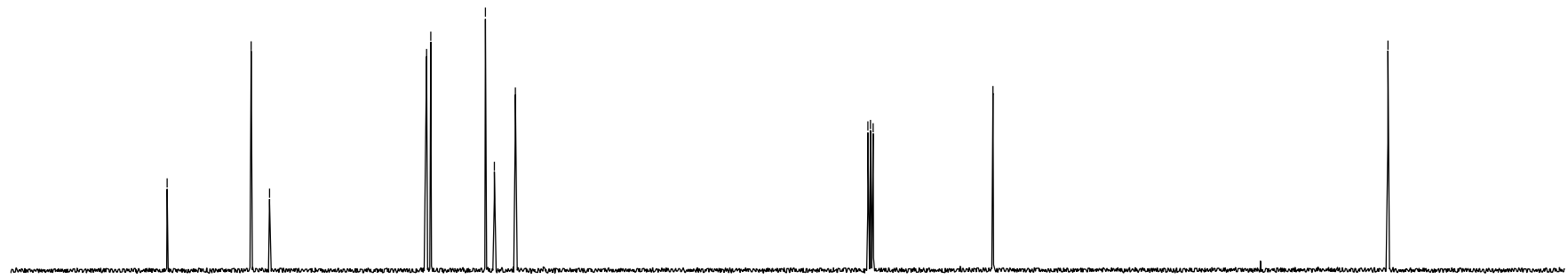

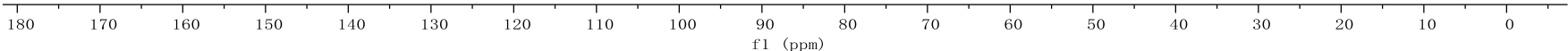




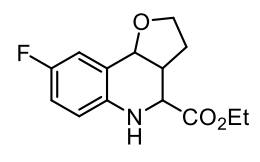

3, Isomer 1 (less polar) $\left(\mathrm{CDCl}_{3}, 400 \mathrm{MHz}\right)$

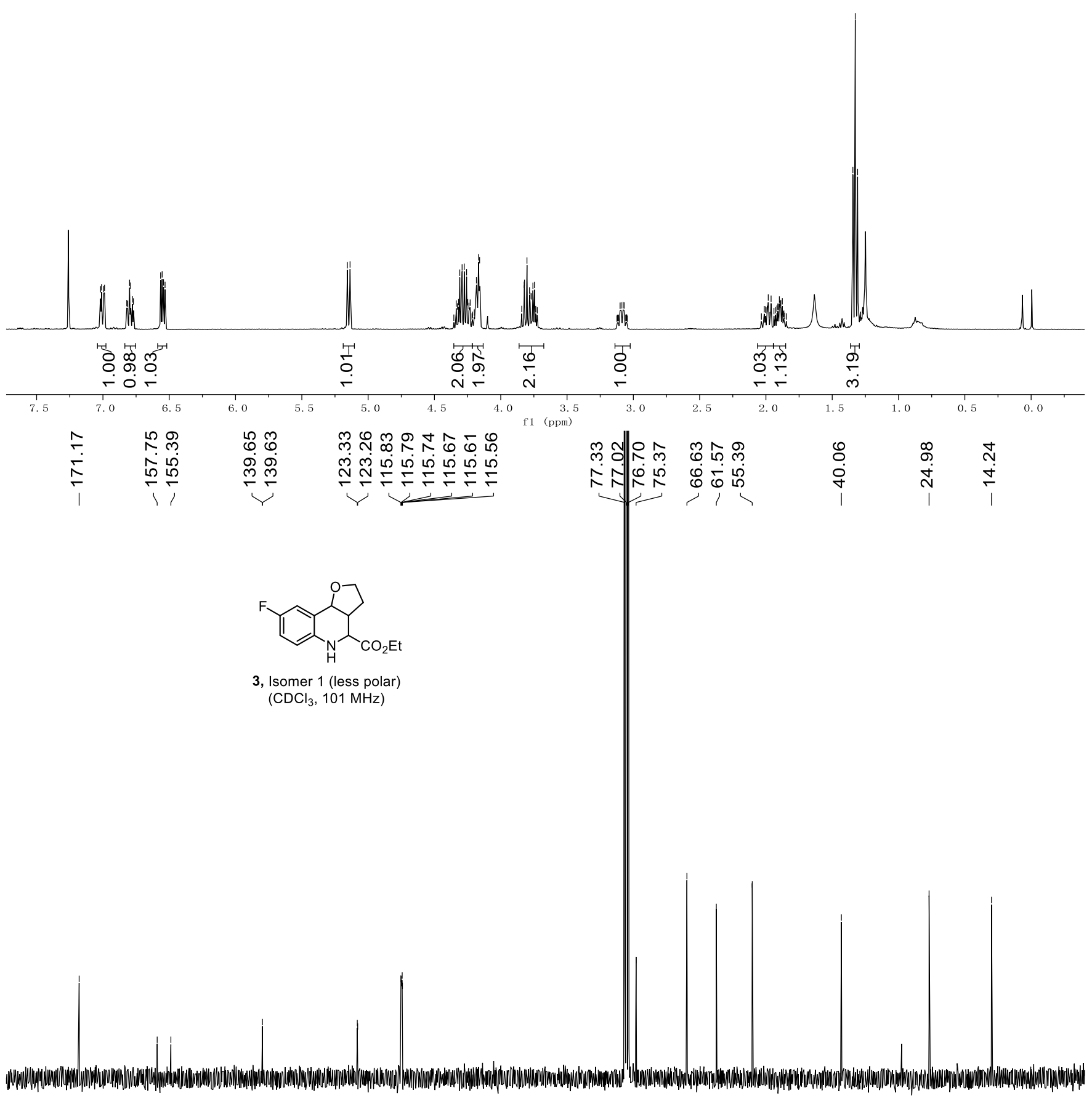

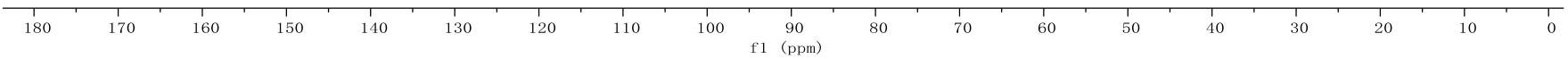


จุ๊

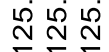

$\underbrace{1 \top \tau}$

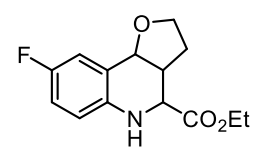

3, Isomer 1 (less polar)

$\left(\mathrm{CDCl}_{3}, 376 \mathrm{MHz}\right)$

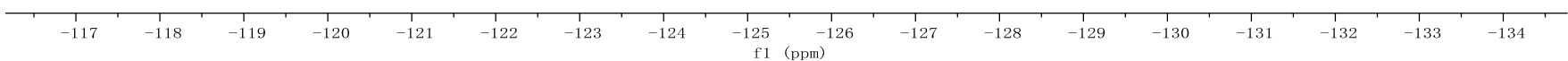




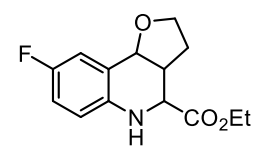

3, Isomer 2 (more polar)

$\left(\mathrm{CDCl}_{3}, 400 \mathrm{MHz}\right)$
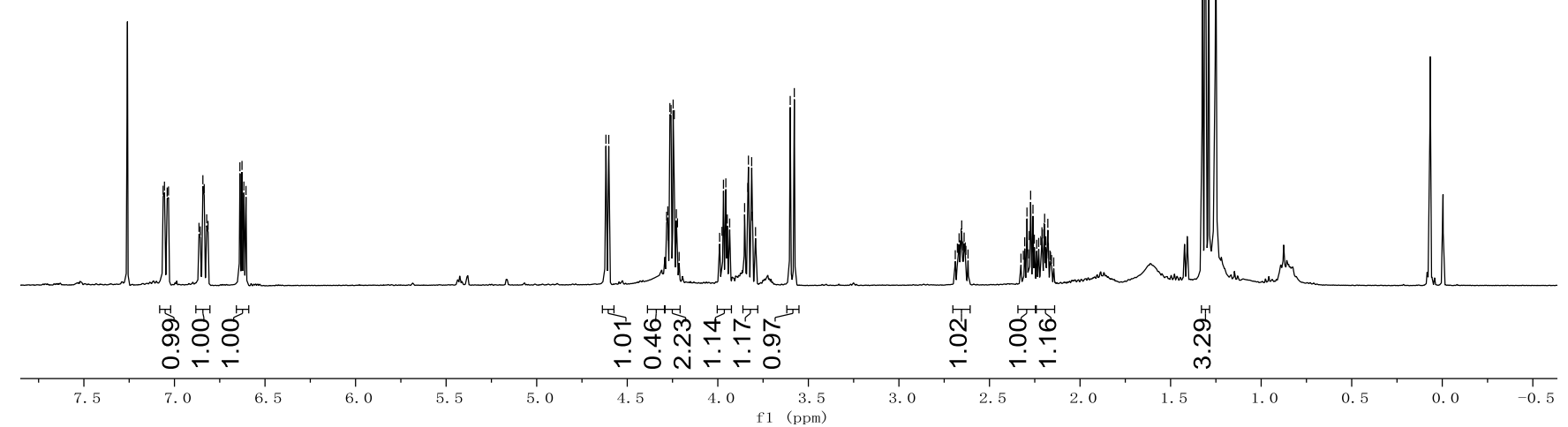

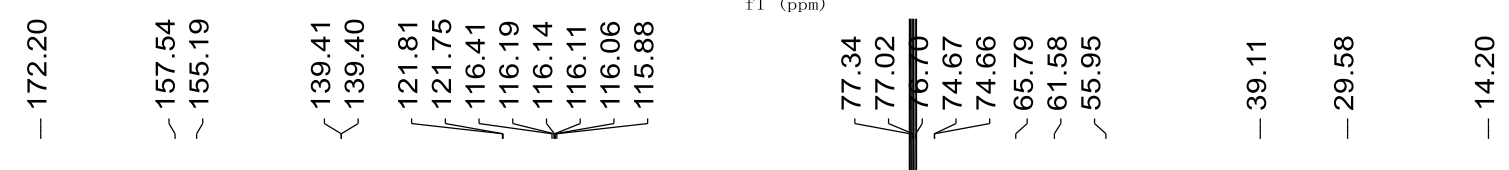

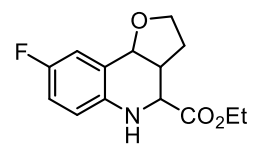

3, Isomer 2 (more polar)

$\left(\mathrm{CDCl}_{3}, 101 \mathrm{MHz}\right)$
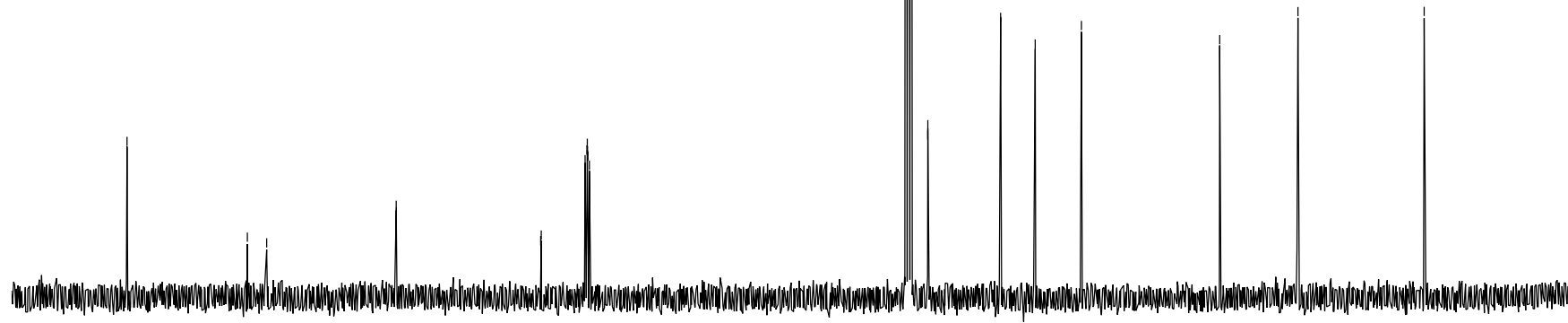

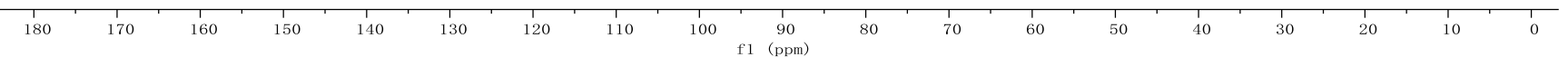


จุ

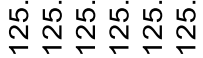

$\underbrace{1}$

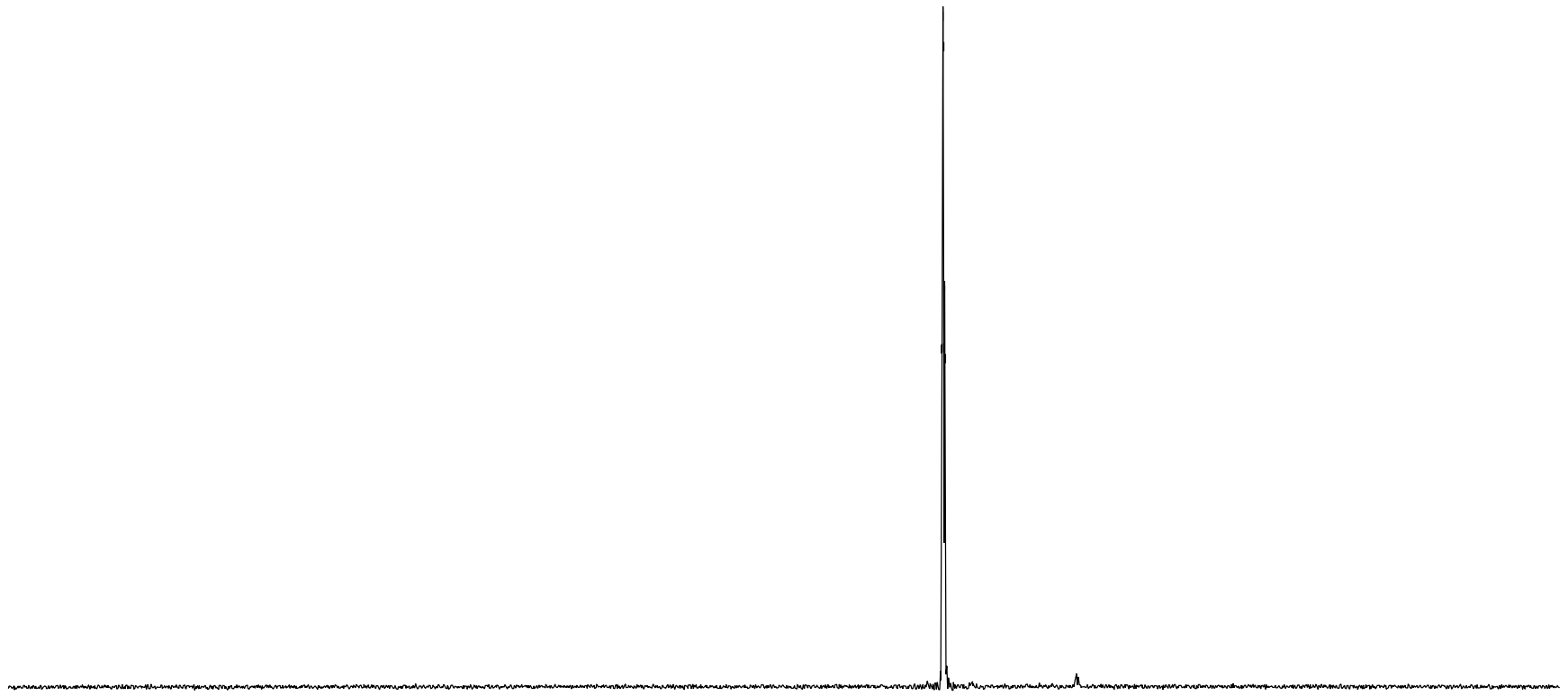

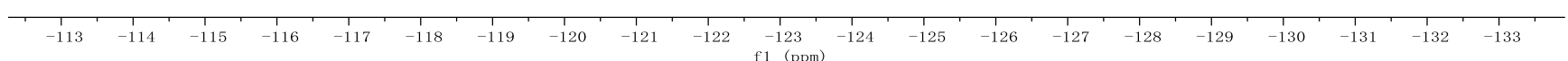




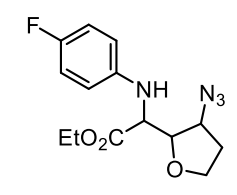

4aa, Isomer 1 (less polar)

$\left(\mathrm{CDCl}_{3}, 600 \mathrm{MHz}\right)$
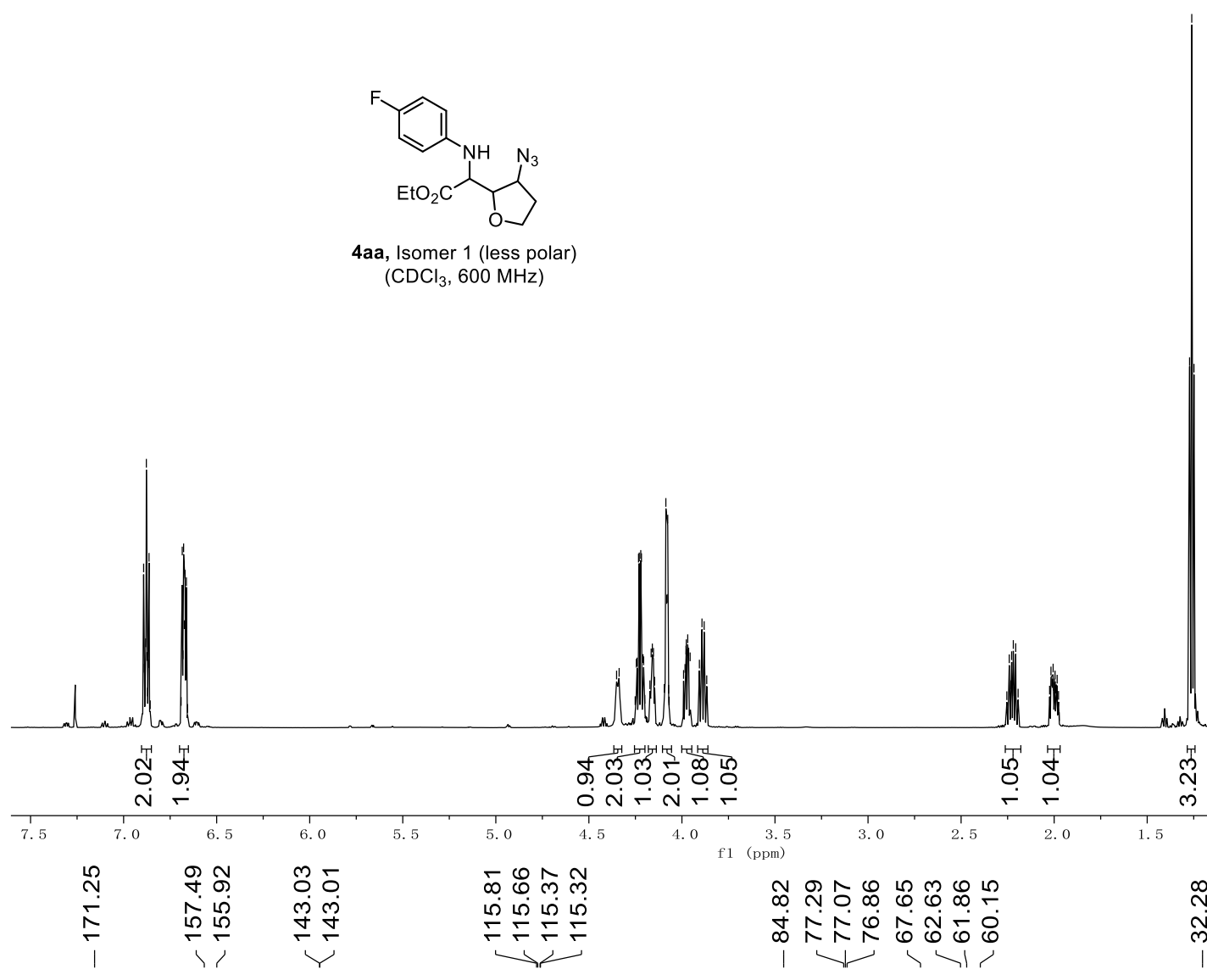

㔯 导

虫
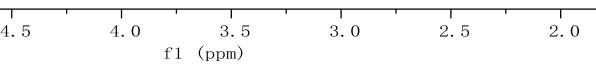

1.5

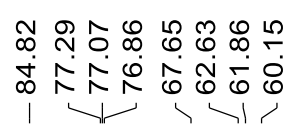

\begin{tabular}{|c|c|}
\hline & $\stackrel{\infty}{N}$ \\
\hline & vै \\
\hline
\end{tabular}

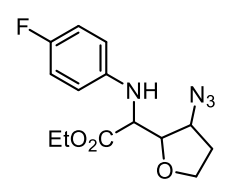

4aa, Isomer 1 (less polar) $\left(\mathrm{CDCl}_{3}, 151 \mathrm{MHz}\right)$

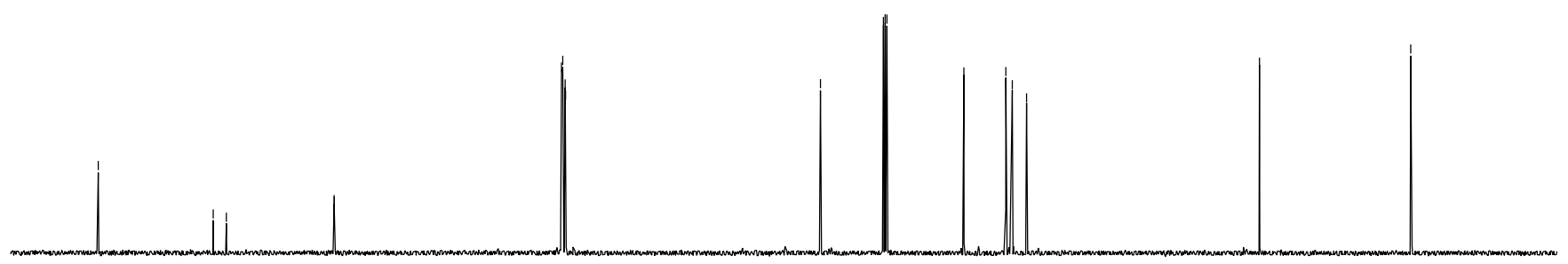

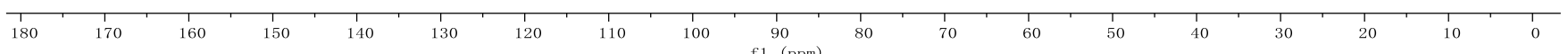




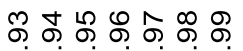

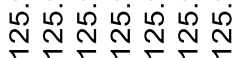

$\underbrace{1}$

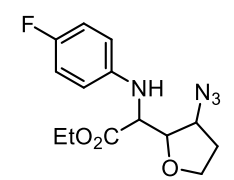

4aa, Isomer 1 (less polar) $\left(\mathrm{CDCl}_{3}, 376 \mathrm{MHz}\right)$

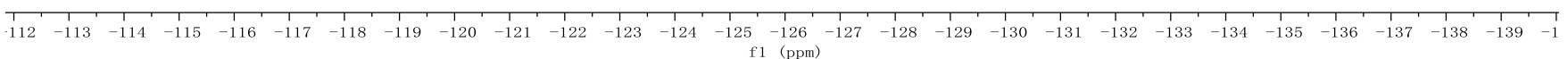




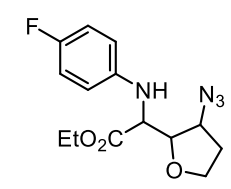

4aa, Isomer 2 (more polar) $\left(\mathrm{CDCl}_{3}, 600 \mathrm{MHz}\right)$
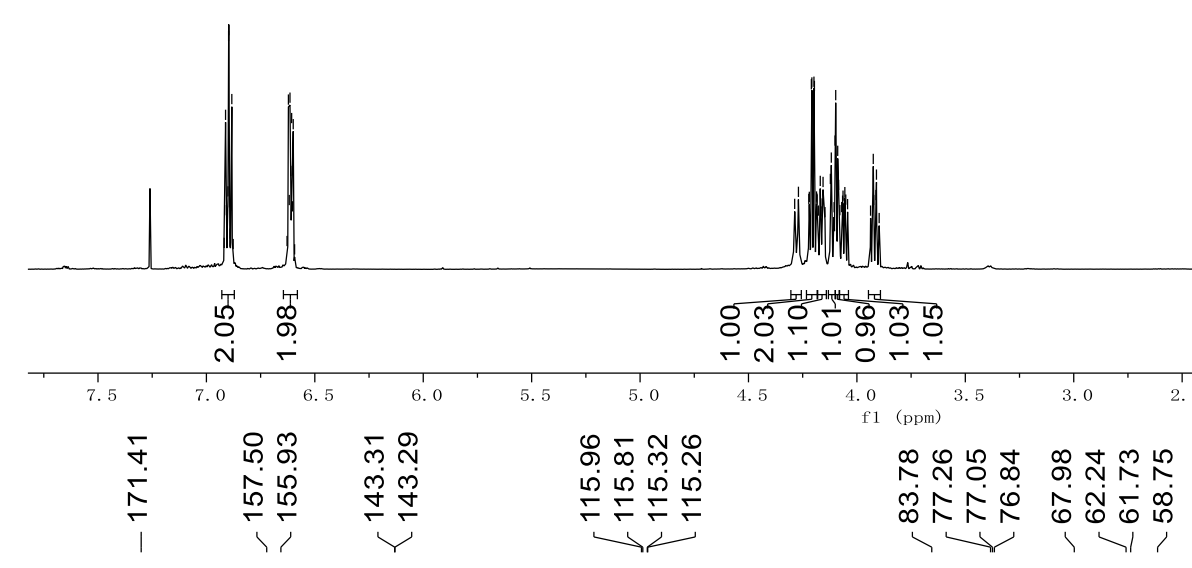

సั่

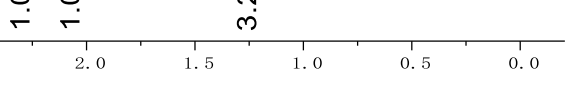

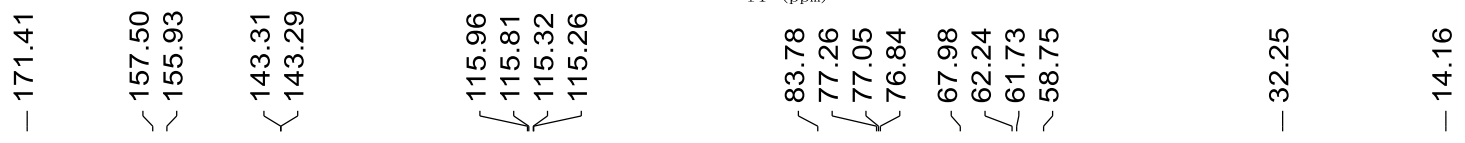

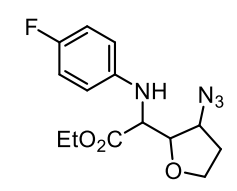

4aa, Isomer 2 (more polar)

$\left(\mathrm{CDCl}_{3}, 151 \mathrm{MHz}\right)$

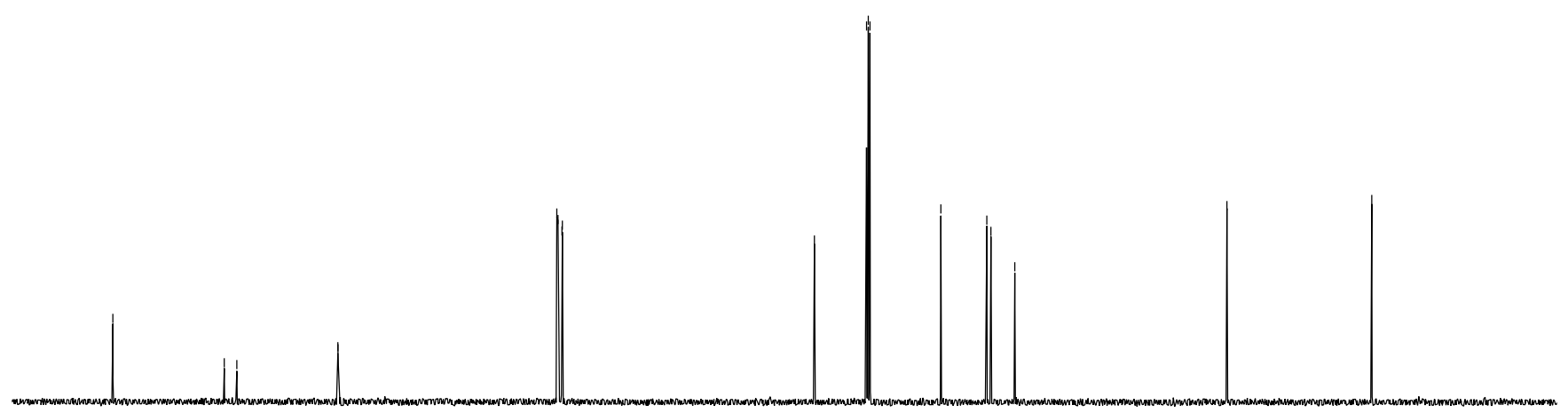

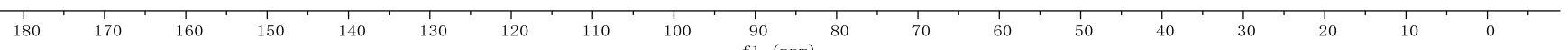




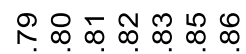

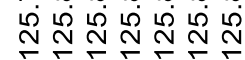
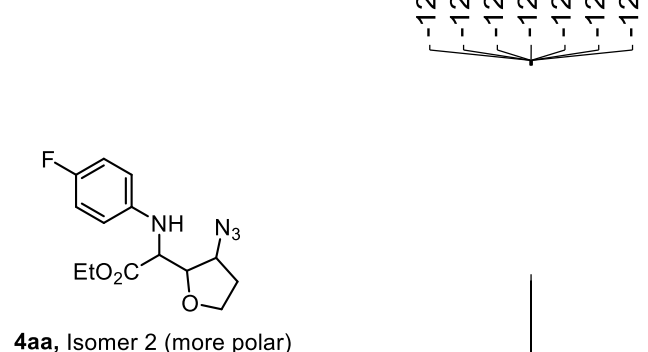

$\left(\mathrm{CDCl}_{3}, 376 \mathrm{MHz}\right)$

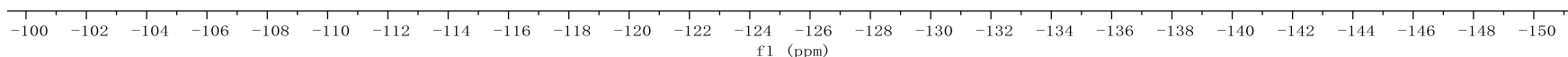



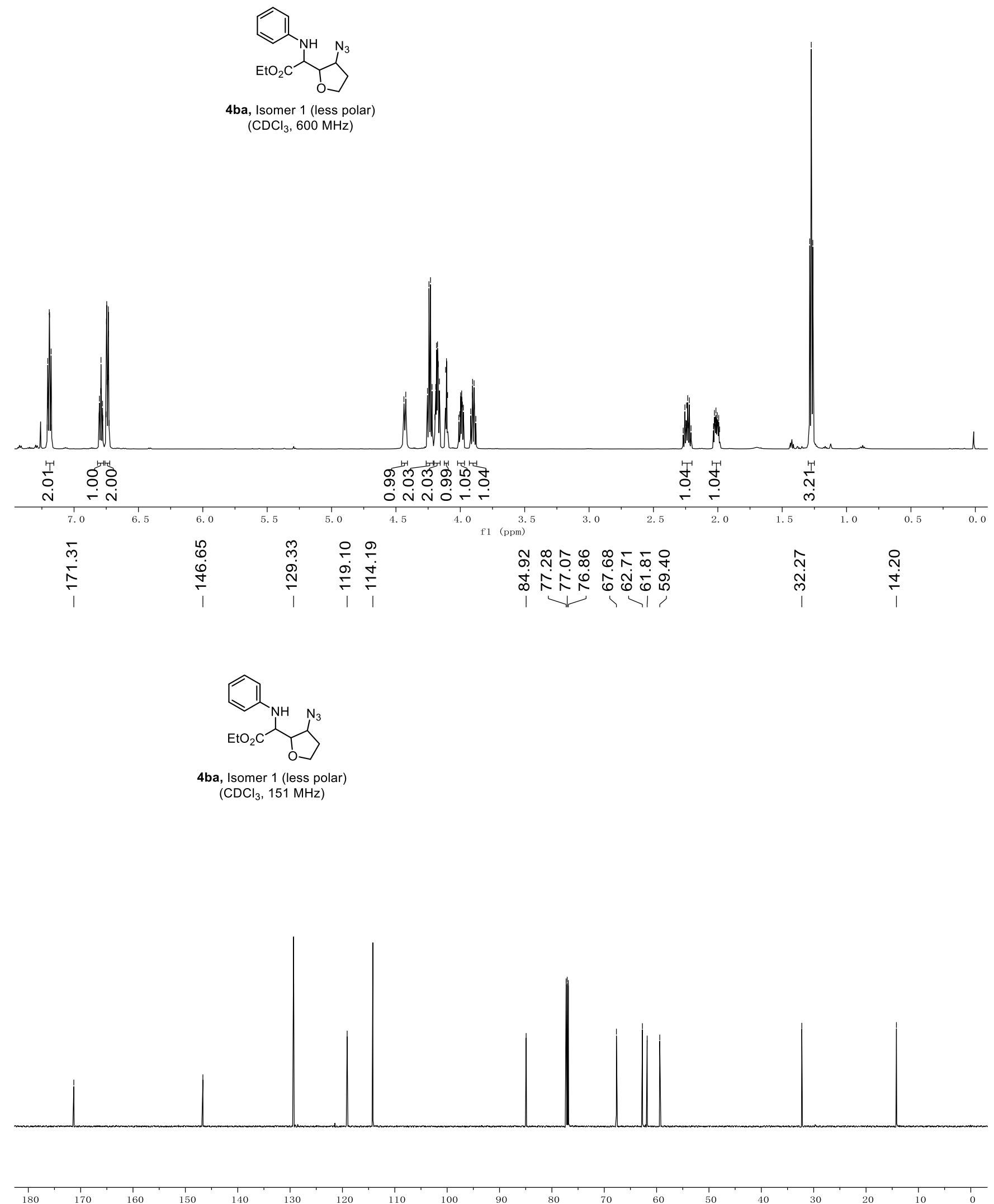

f1 $\stackrel{90}{(\mathrm{ppm})}$

$80+70$

$20110+1$ 


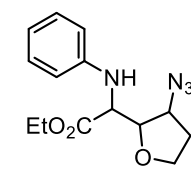

4ba, Isomer 2 (more polar) $\left(\mathrm{CDCl}_{3}, 600 \mathrm{MHz}\right)$
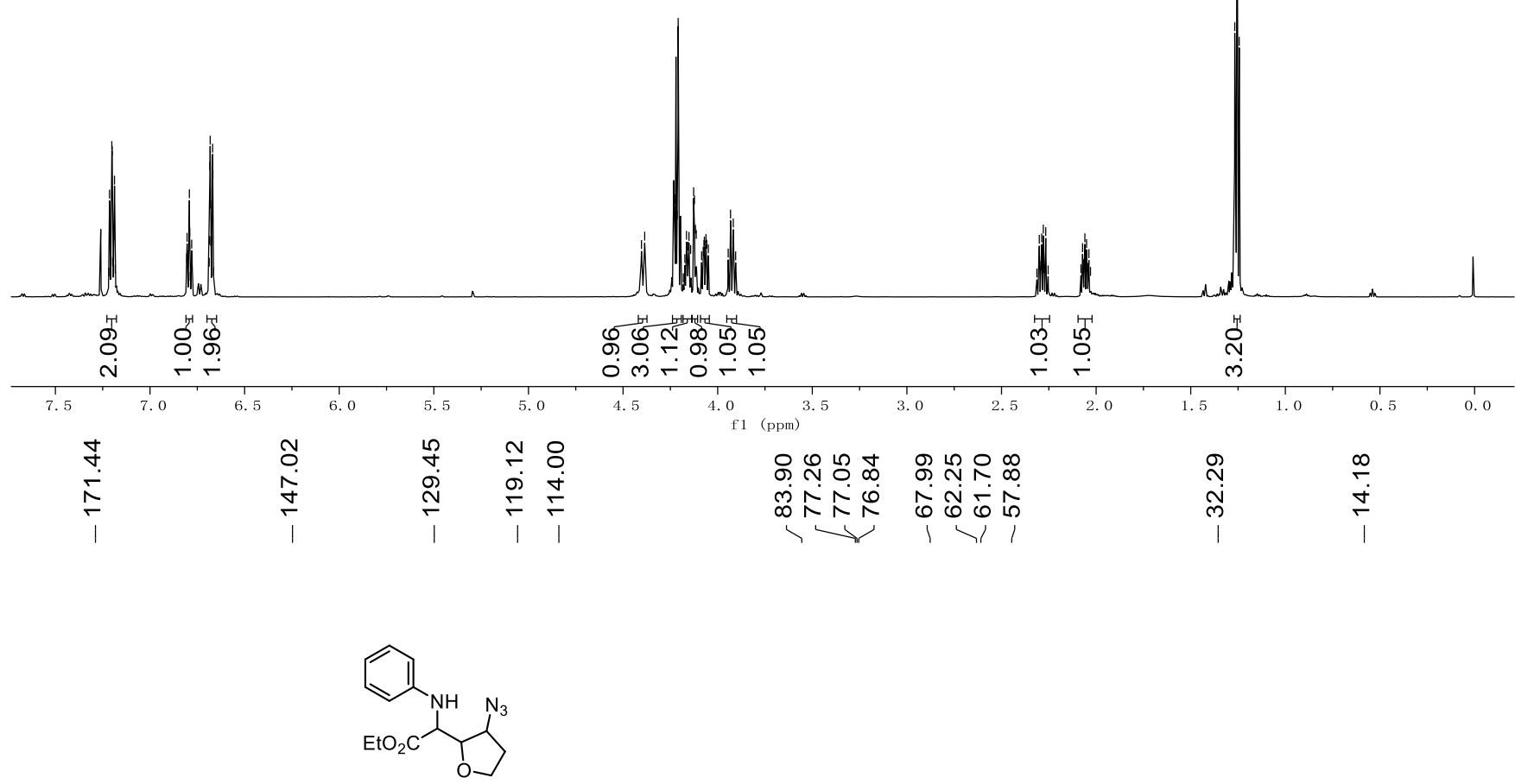

4ba, Isomer 2 (more polar) $\left(\mathrm{CDCl}_{3}, 151 \mathrm{MHz}\right)$

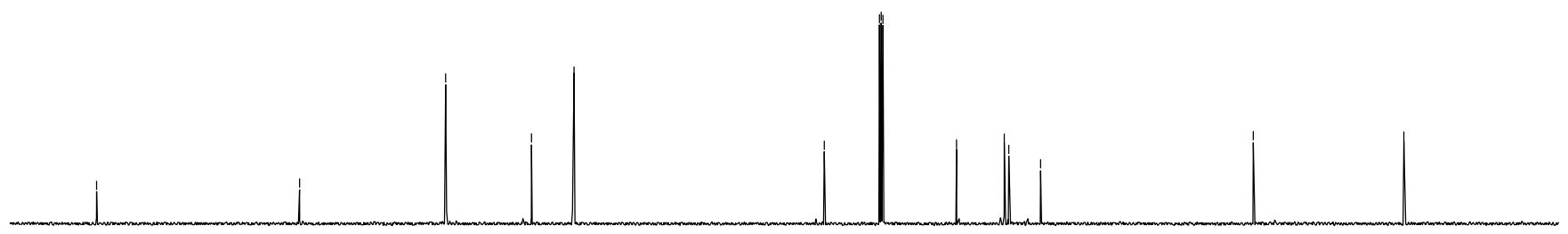

T 180

$170 \quad 160$

150

f1 $1 \mathrm{ppm})$ 

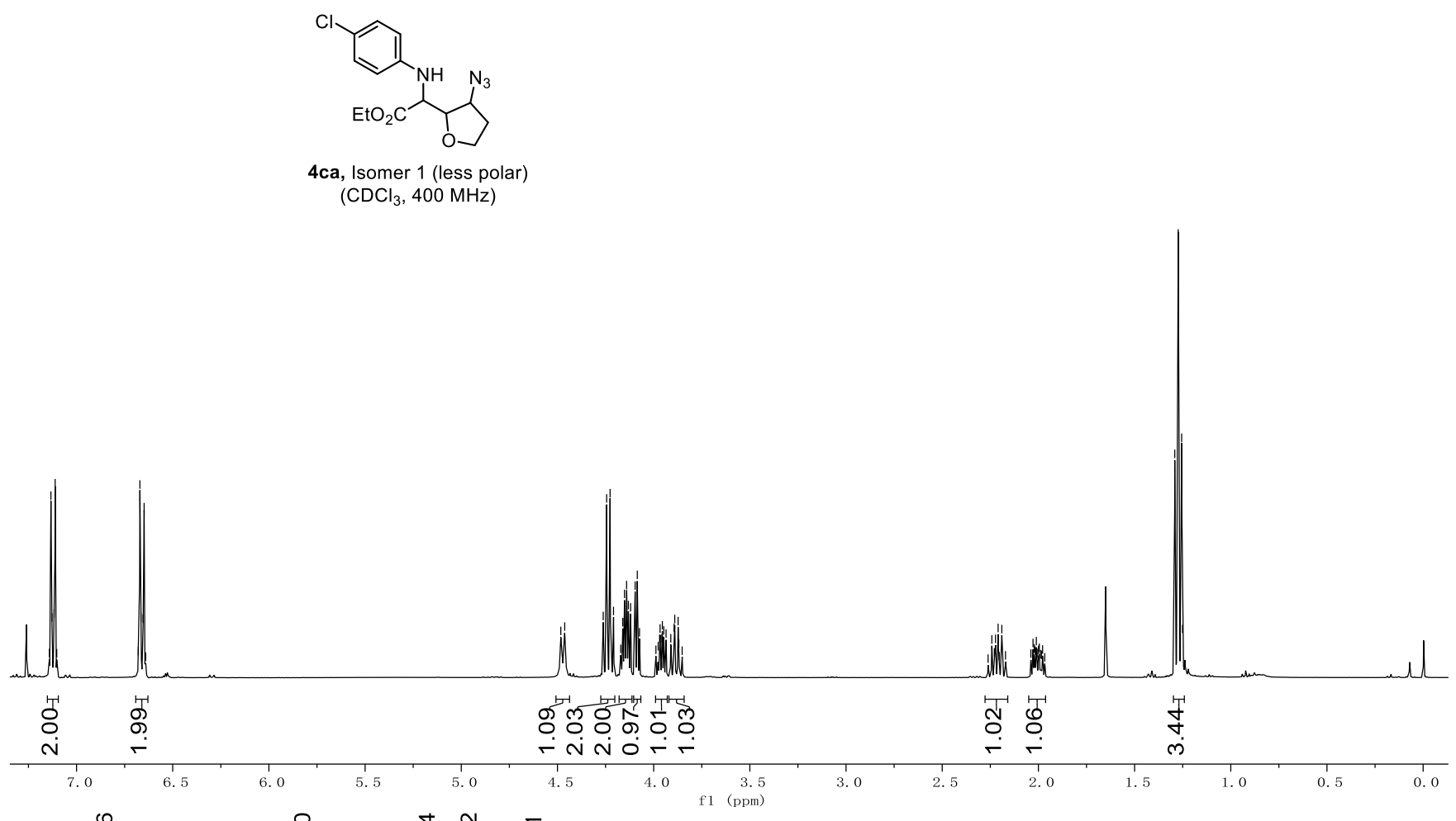

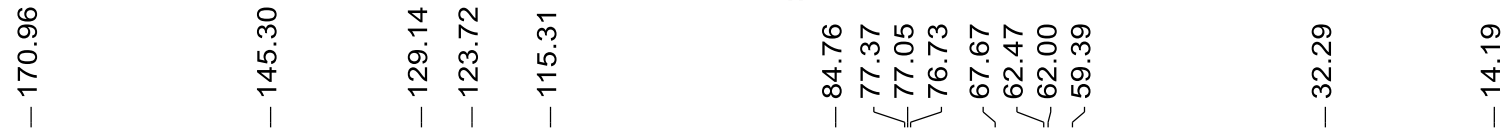

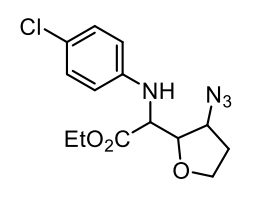

4ca, Isomer 1 (less polar) $\left(\mathrm{CDCl}_{3}, 101 \mathrm{MHz}\right)$

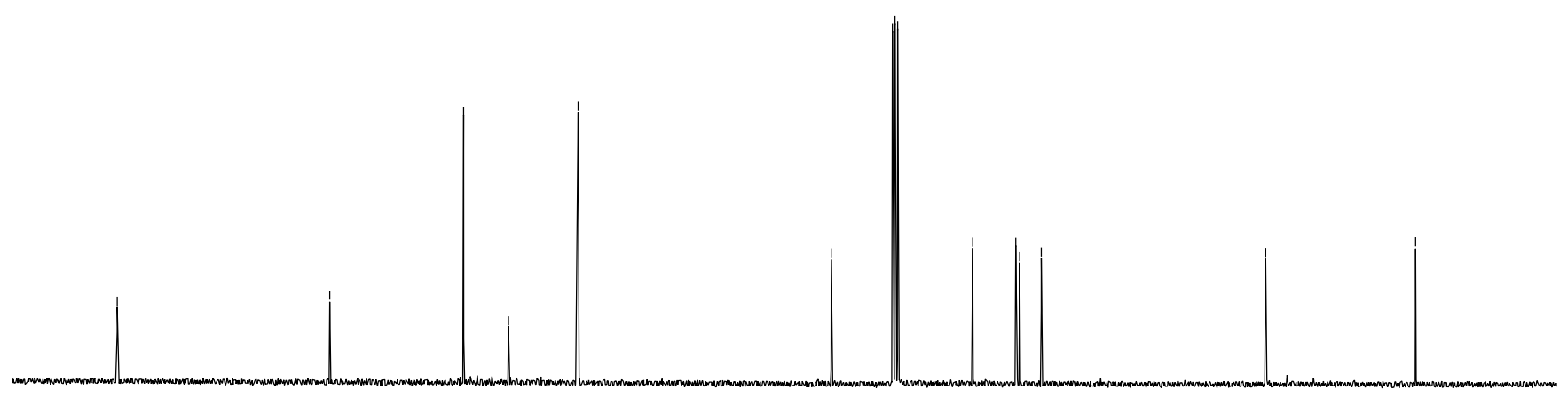

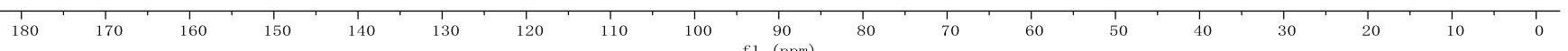




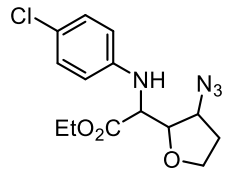

4ca, Isomer 2 (more polar) $\left(\mathrm{CDCl}_{3}, 400 \mathrm{MHz}\right)$
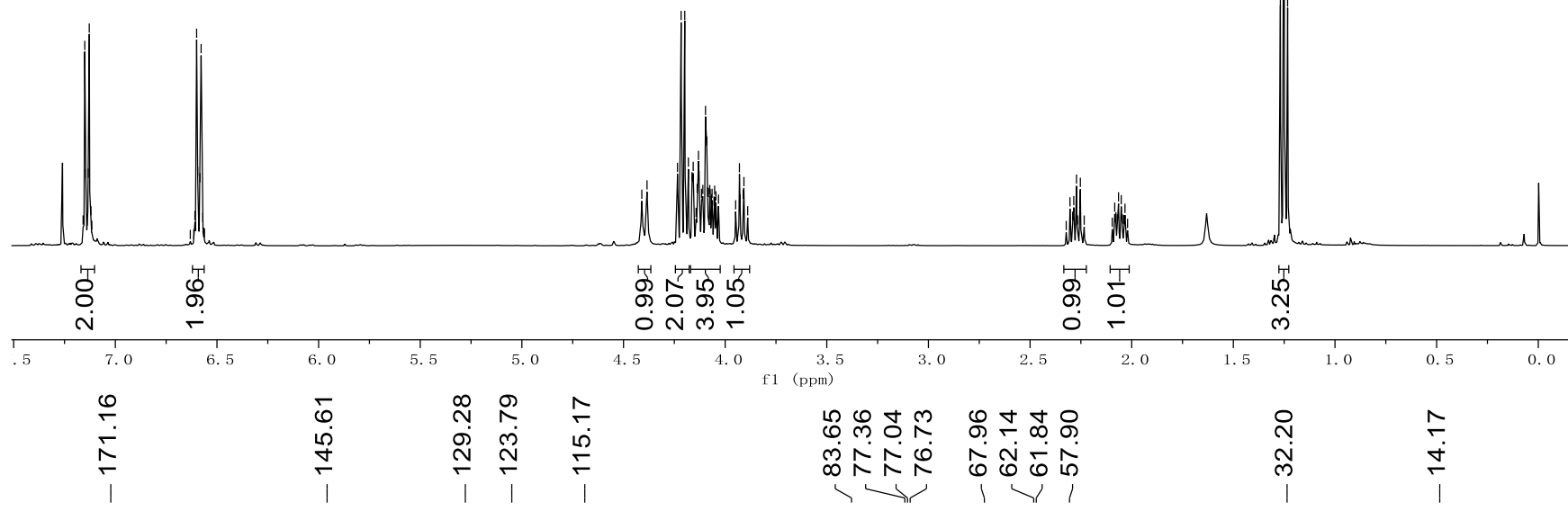

'T

กับ

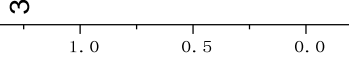

采令

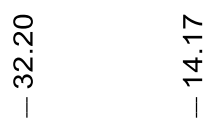

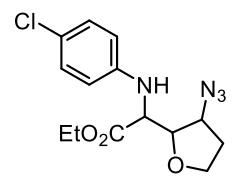

4ca, Isomer 2 (more polar)

$\left(\mathrm{CDCl}_{3}, 101 \mathrm{MHz}\right)$

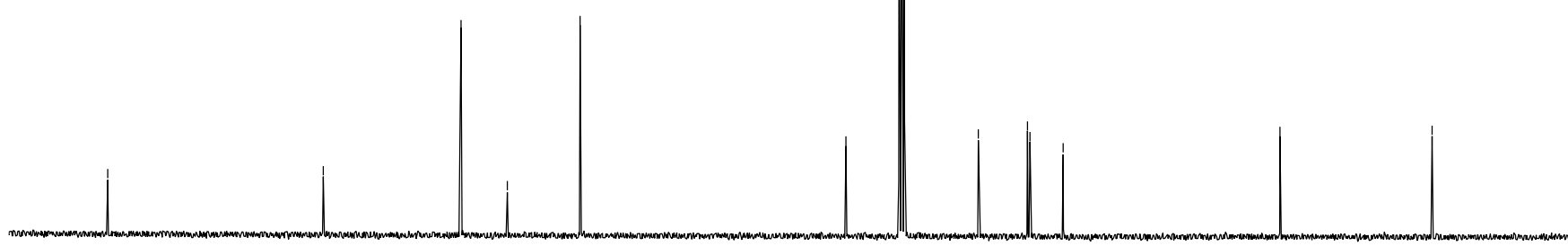

$180 \quad 170 \quad 160 \quad 150 \quad 140 \quad 130 \quad 120$ f1 $(\mathrm{ppm})$ 


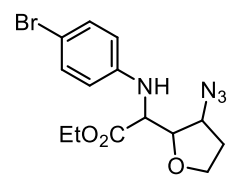

4da, Isomer 1 (less polar) $\left(\mathrm{CDCl}_{3}, 400 \mathrm{MHz}\right)$

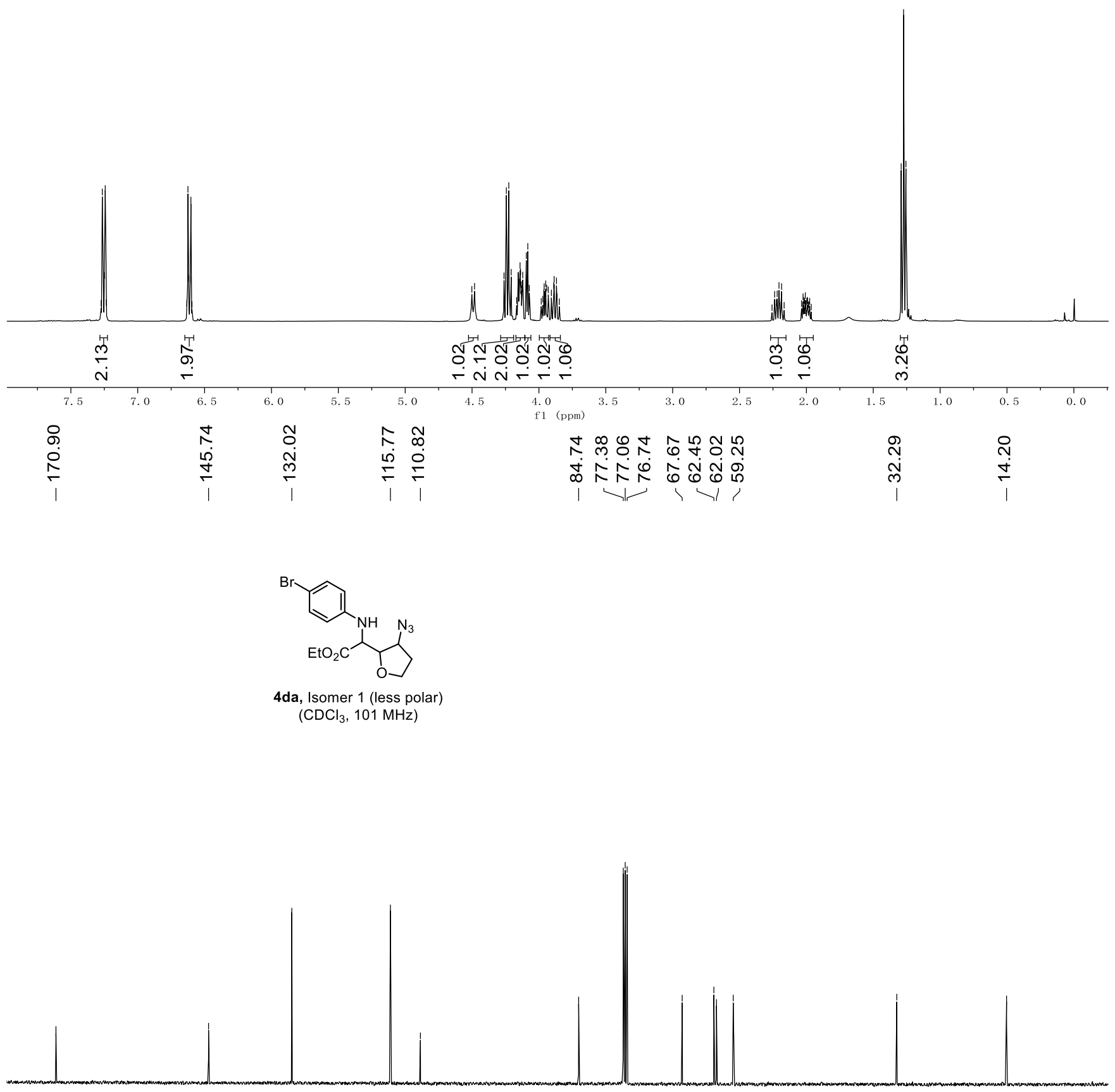

170

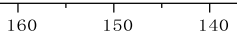

$110 \quad 100$

f1 (ppm)

1
$80 \quad 70$

60

50 


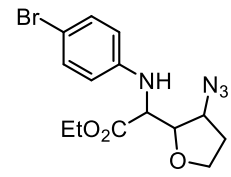

4da, Isomer 2 (more polar) $\left(\mathrm{CDCl}_{3}, 400 \mathrm{MHz}\right)$
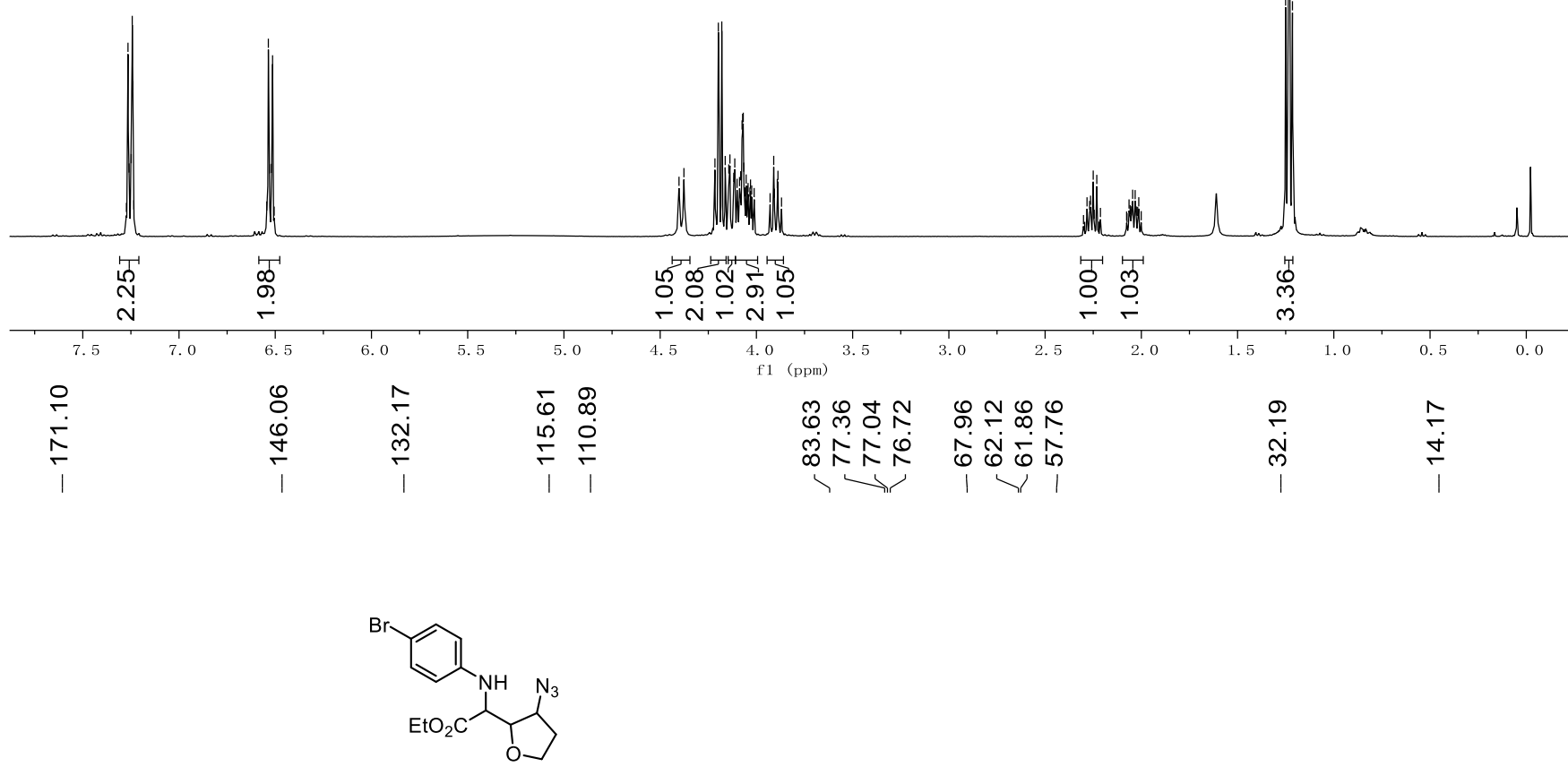

4da, Isomer 2 (more polar) $\left(\mathrm{CDCl}_{3}, 101 \mathrm{MHz}\right.$

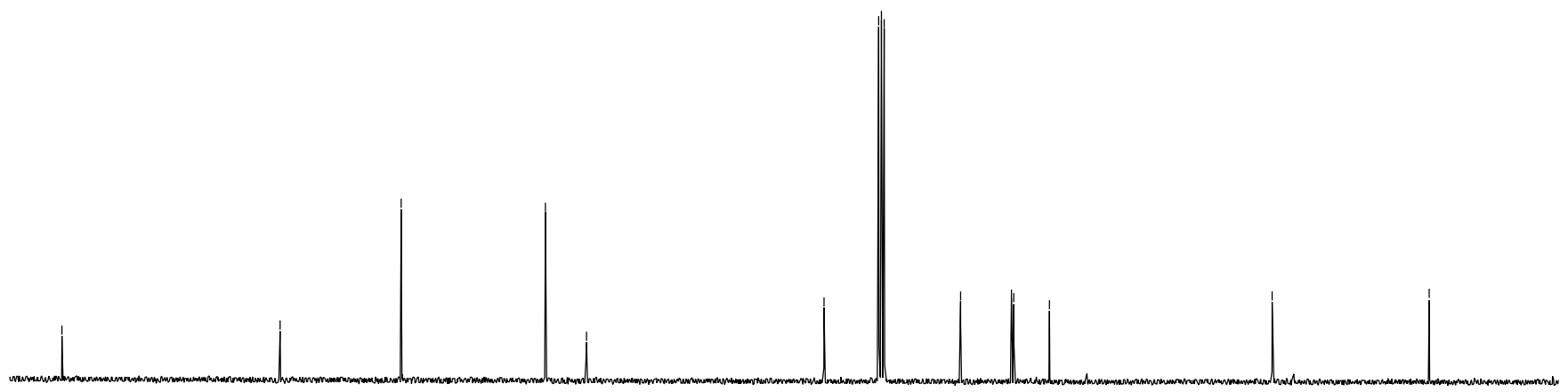

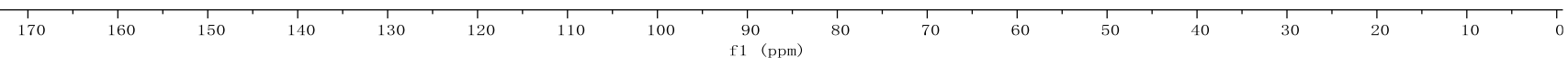




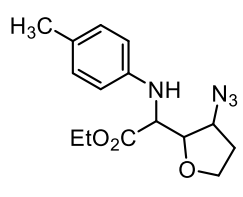

4ea, Isomer 1 (less polar)

$\left(\mathrm{CDCl}_{3}, 600 \mathrm{MHz}\right)$
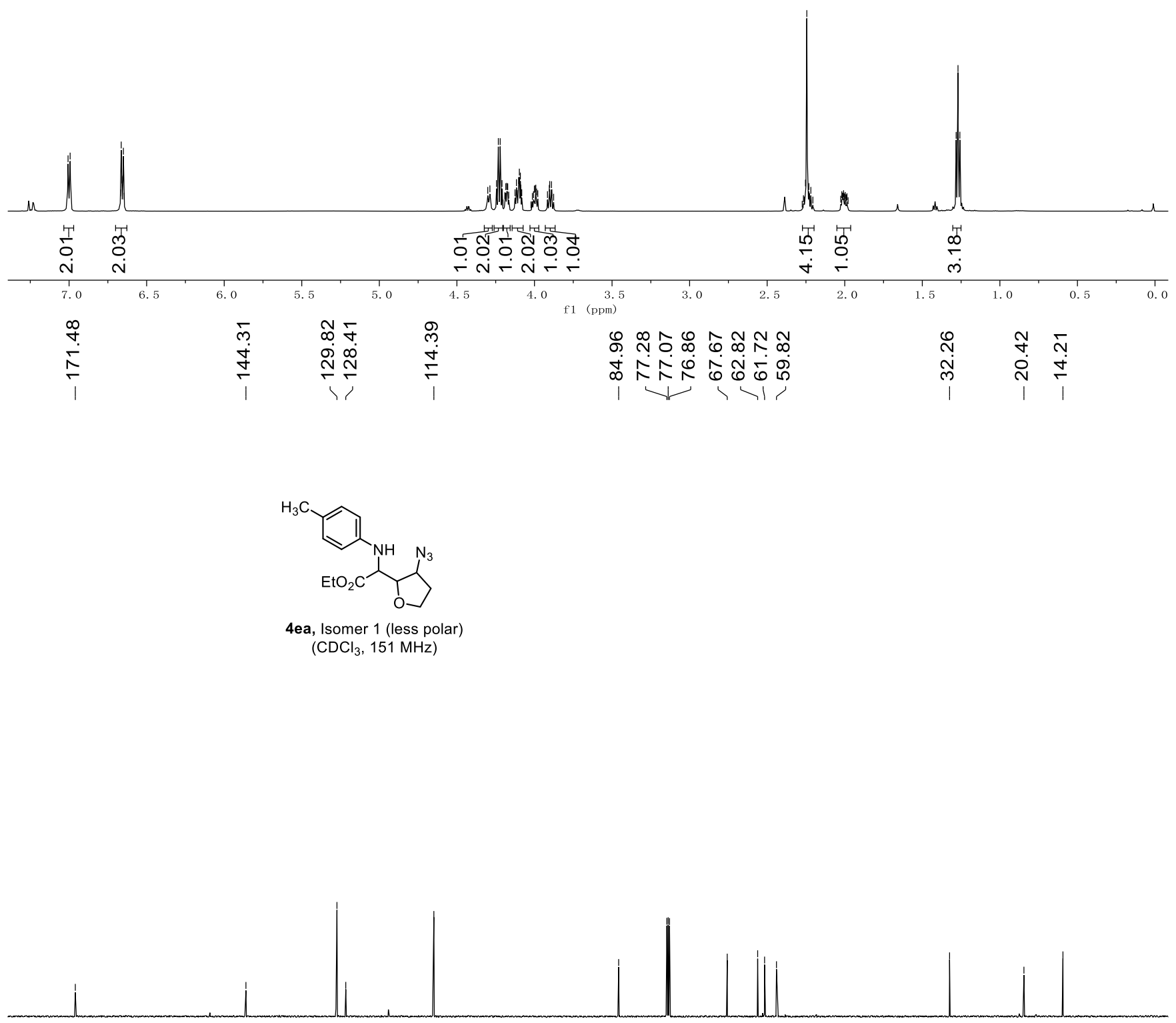

$\frac{1}{180}$

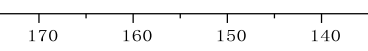

$140 \quad 130$

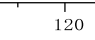

110

f1 $\stackrel{90}{(\mathrm{ppm})}$ 


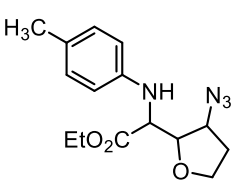

4ea, Isomer 2 (more polar)

$\left(\mathrm{CDCl}_{3}, 400 \mathrm{MHz}\right)$
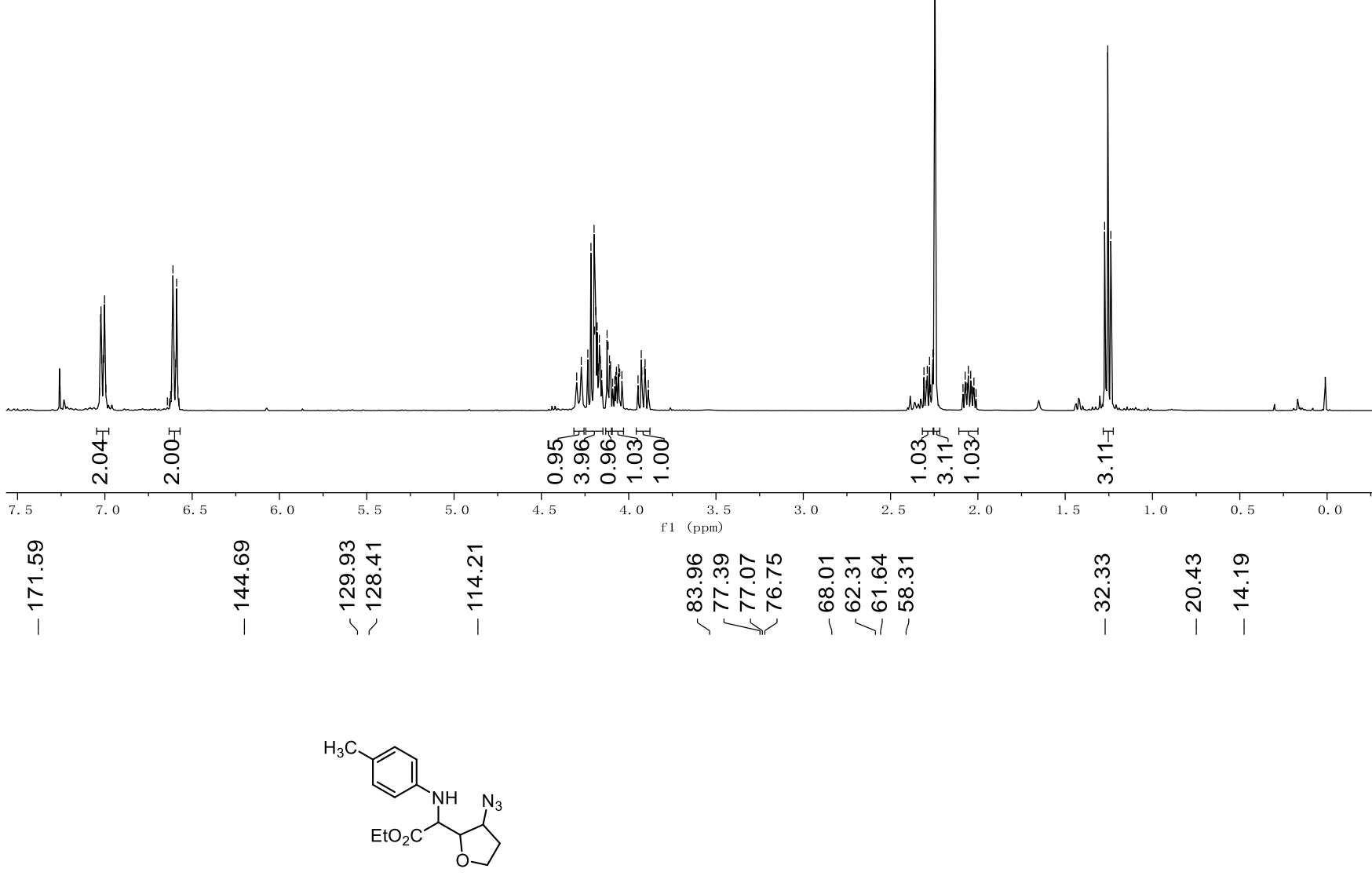

4ea, Isomer 2 (more polar)

$\left(\mathrm{CDCl}_{3}, 101 \mathrm{MHz}\right)$

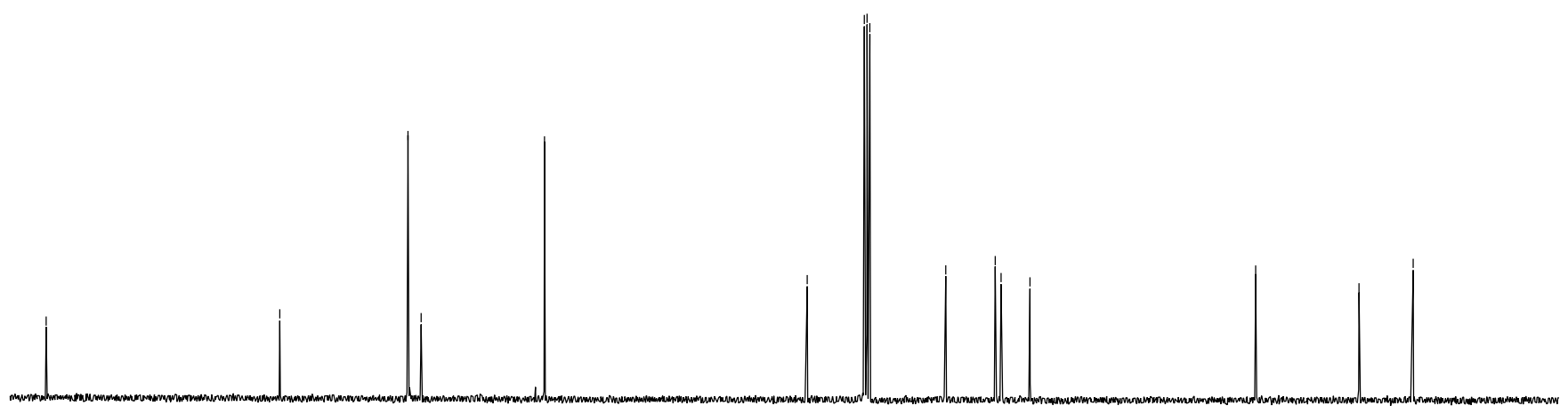

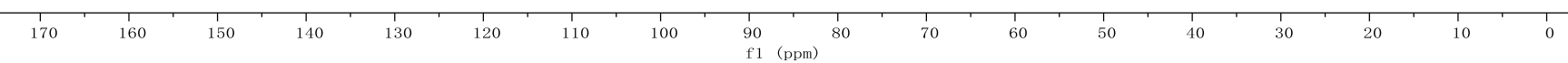



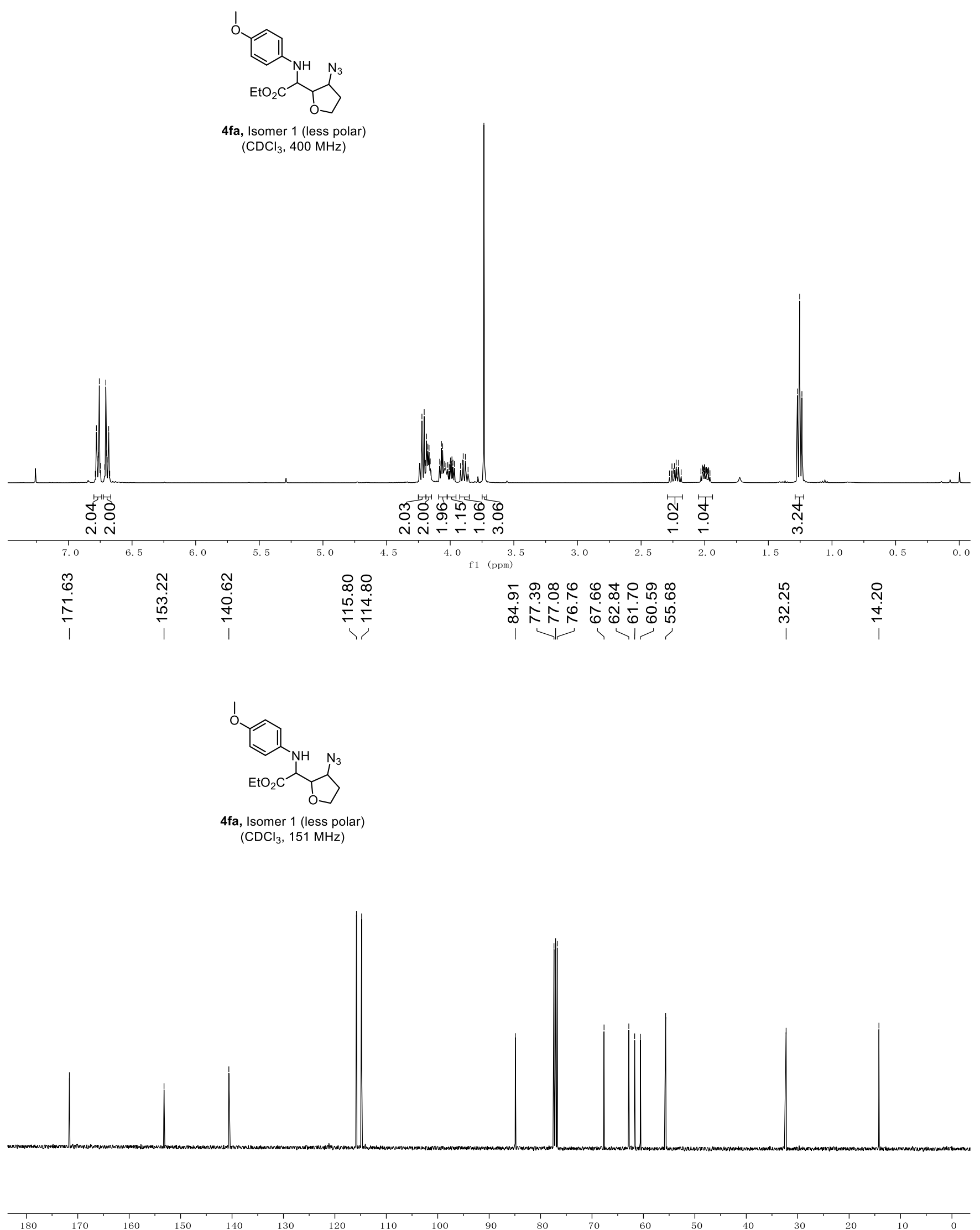

90

80

$70 \quad 60$




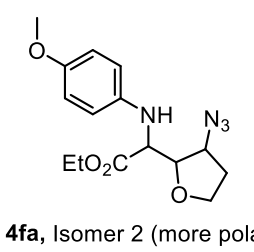

$\left(\mathrm{CDCl}_{3}, 400 \mathrm{MHz}\right)$

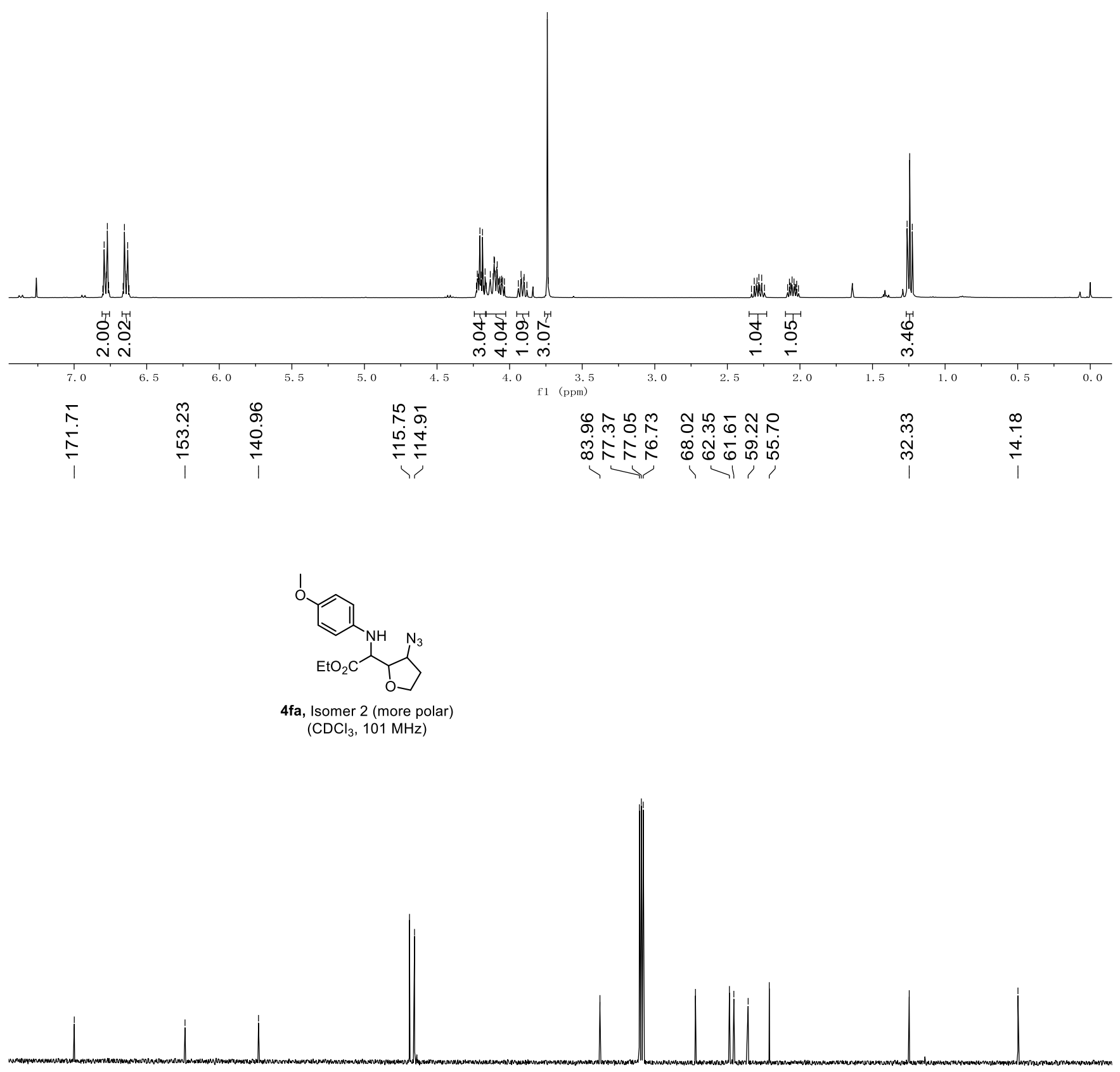




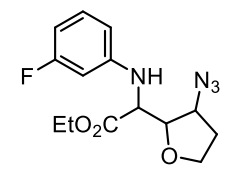

4ga, Isomer 1 (less polar)

$\left(\mathrm{CDCl}_{3}, 400 \mathrm{MHz}\right)$

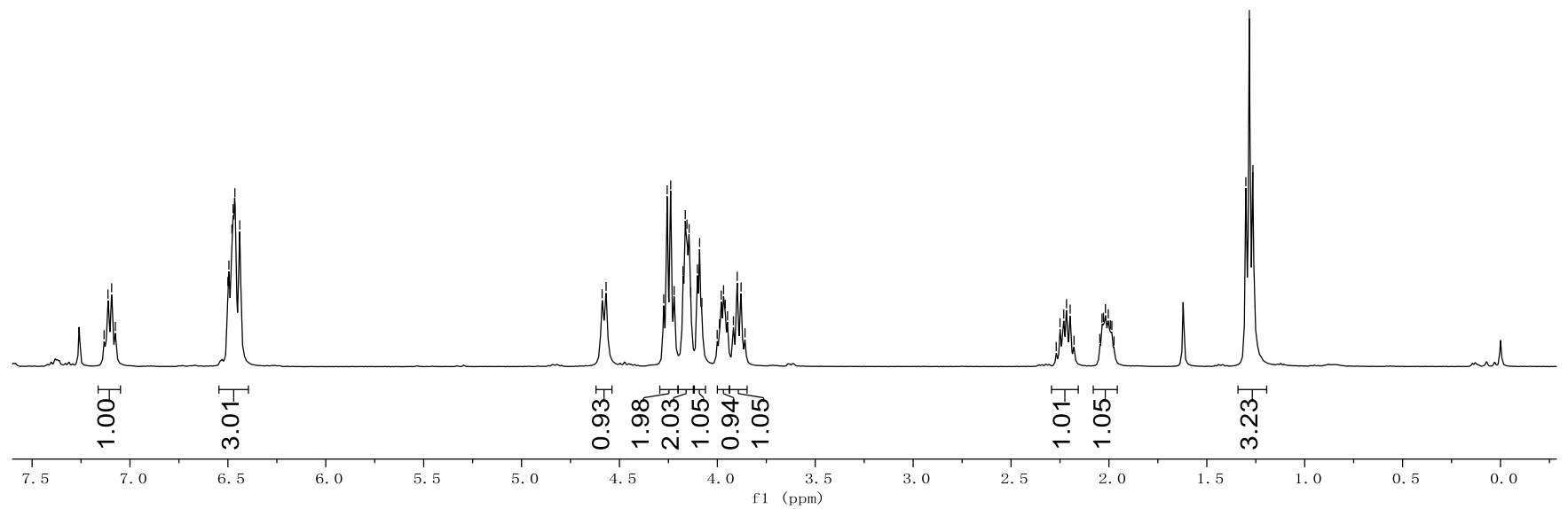

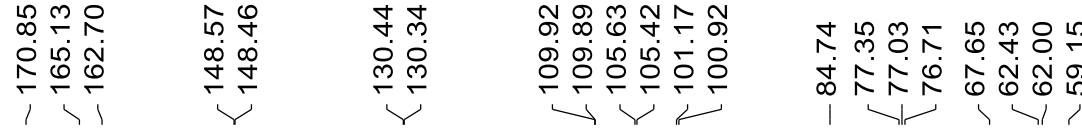

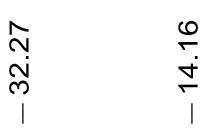

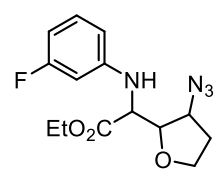

4ga, Isomer 1 (less polar)

$\left(\mathrm{CDCl}_{3}, 101 \mathrm{MHz}\right)$

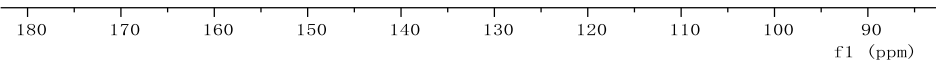




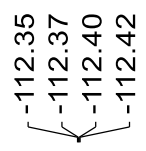

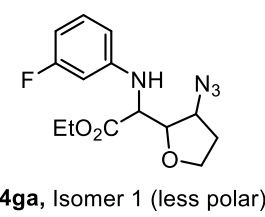

Aga, Isomer 1 (less polar)
$\left(\mathrm{CDCl}_{3}, 376 \mathrm{MHz}\right)$

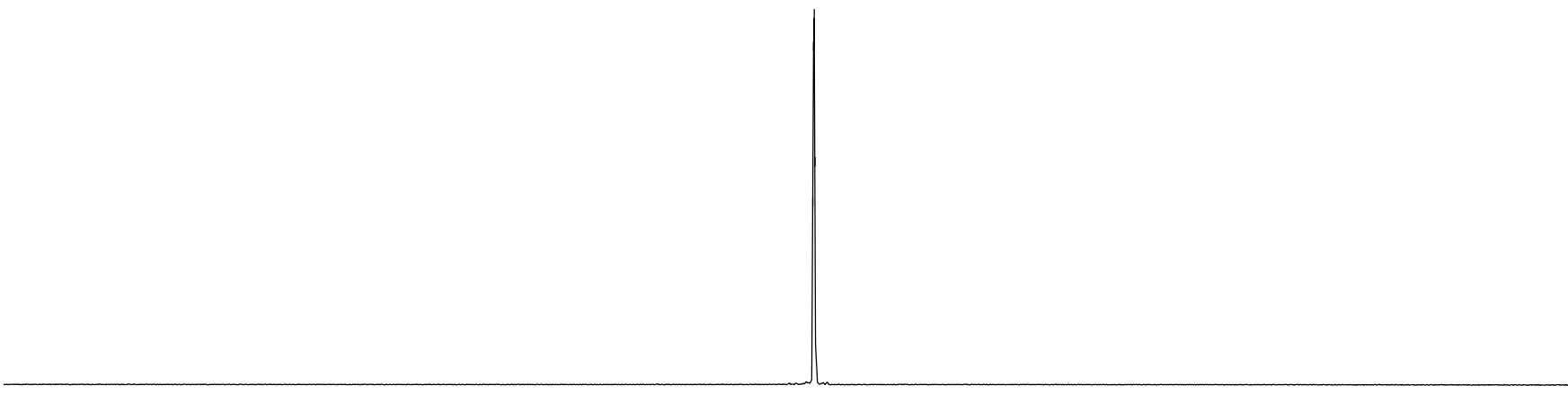

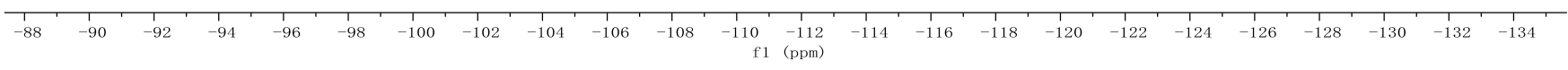

S49 


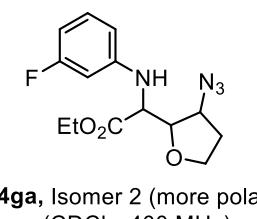

4ga, Isomer 2 (more polar)

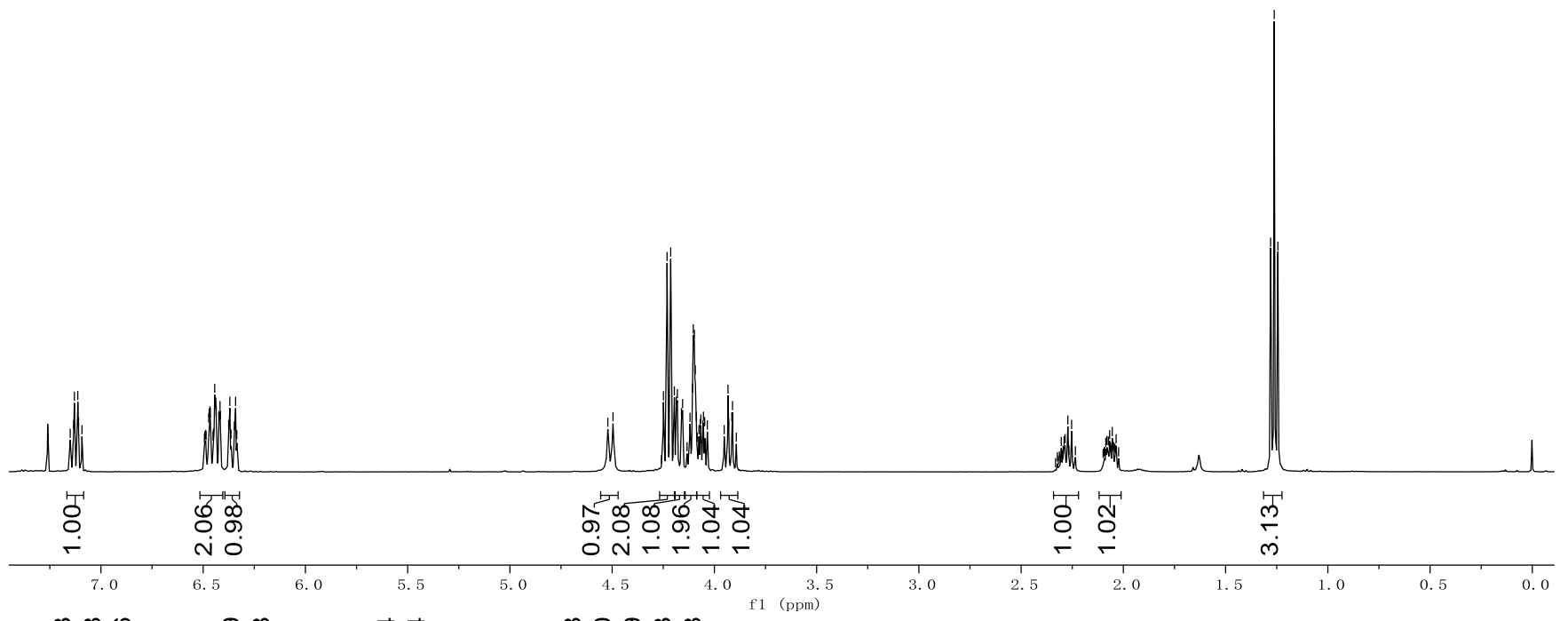

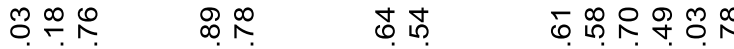

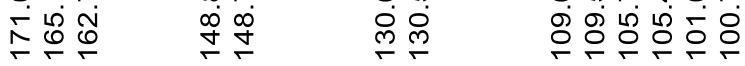

सִ

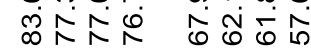

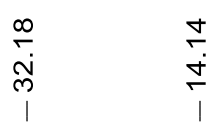

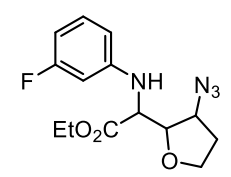

4ga, Isomer 2 (more polar) $\left(\mathrm{CDCl}_{3}, 101 \mathrm{MHz}\right)$

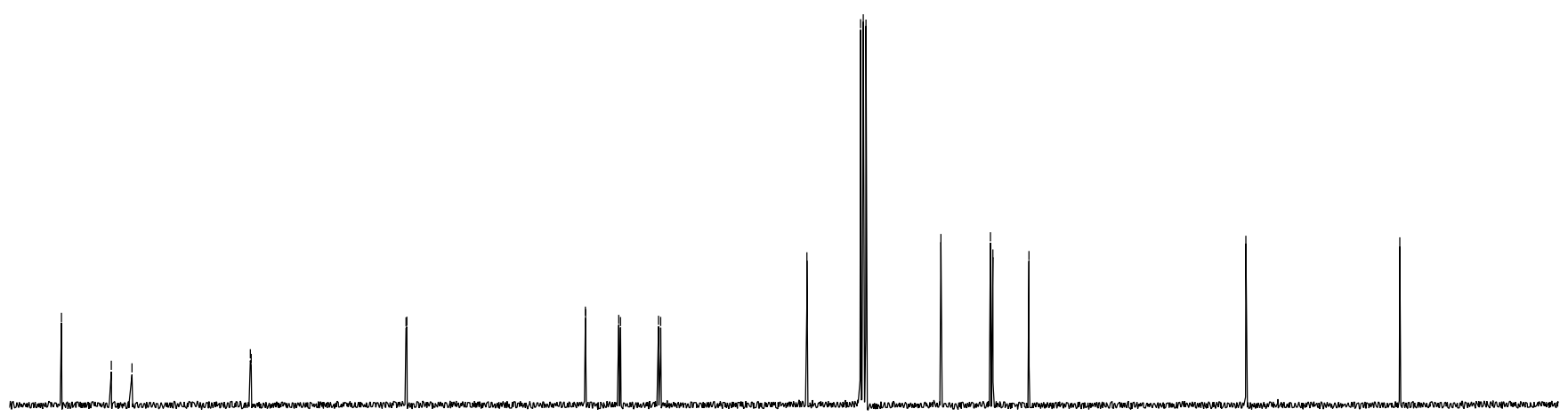

$\begin{array}{rrrrrrrrr}170 & 160 & 150 & 140 & 130 & 120 & 110 & 100 & 90 \\ & & & & & 1 & 1 & 1 & 1\end{array}$ 
$\underbrace{3}$

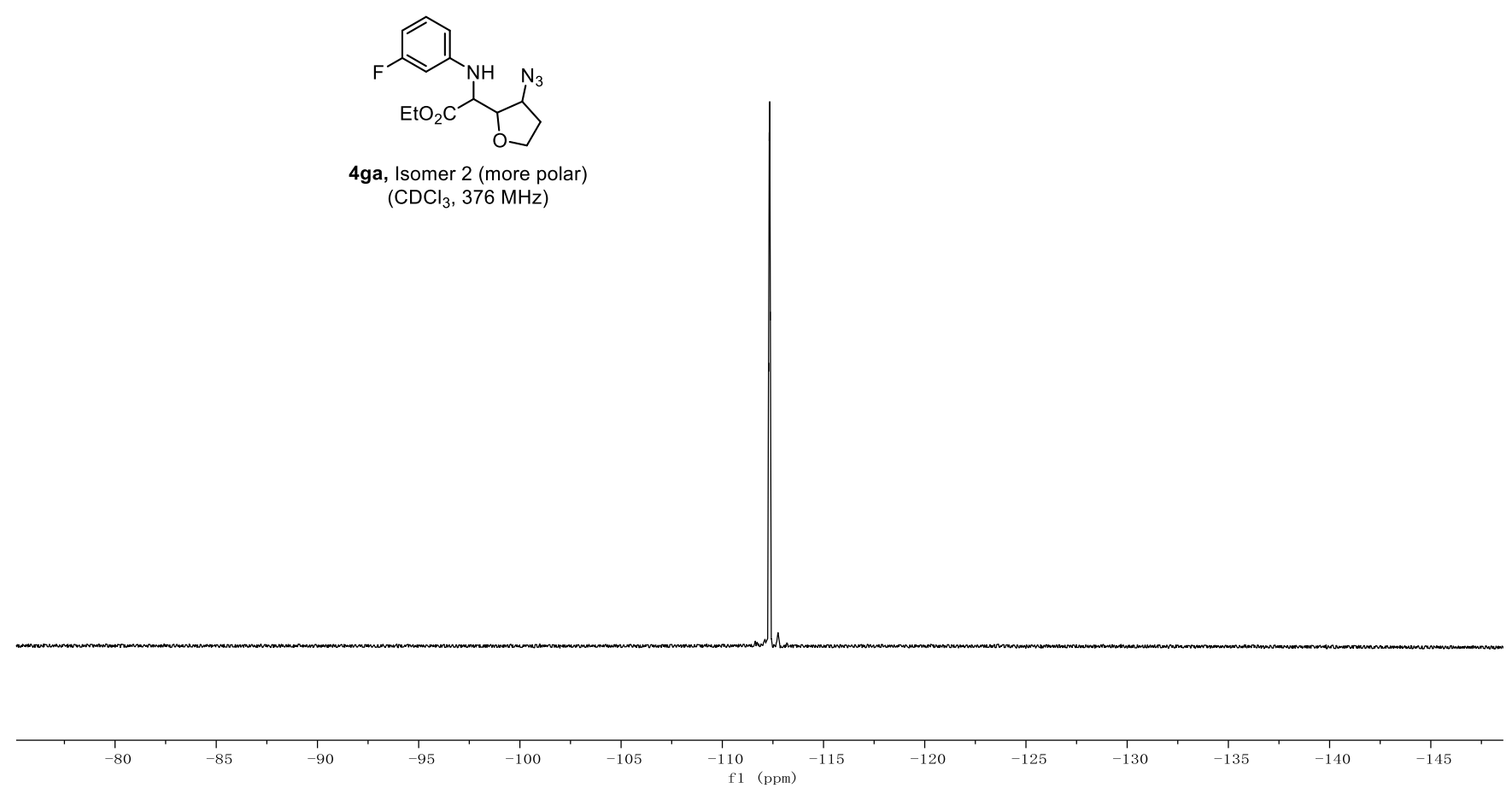

S51 


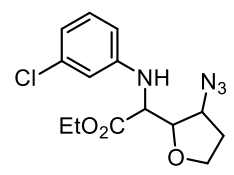

4ha, Isomer 1 (less polar)

$$
\left(\mathrm{CDCl}_{3}, 400 \mathrm{MHz}\right)
$$
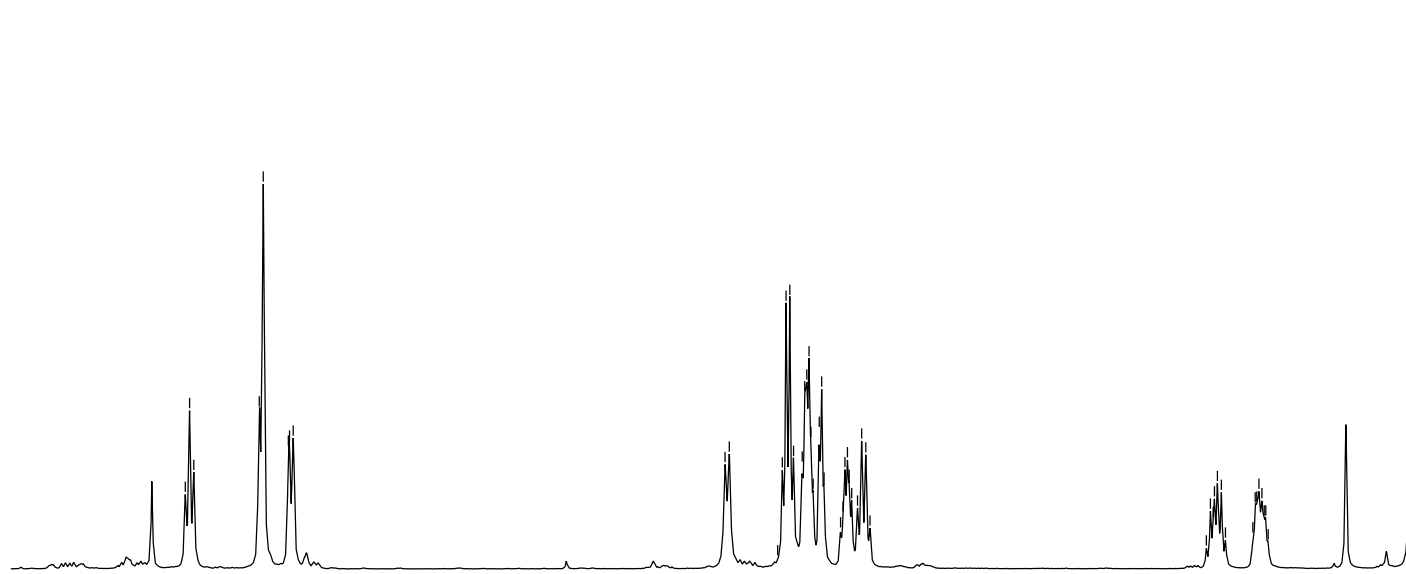

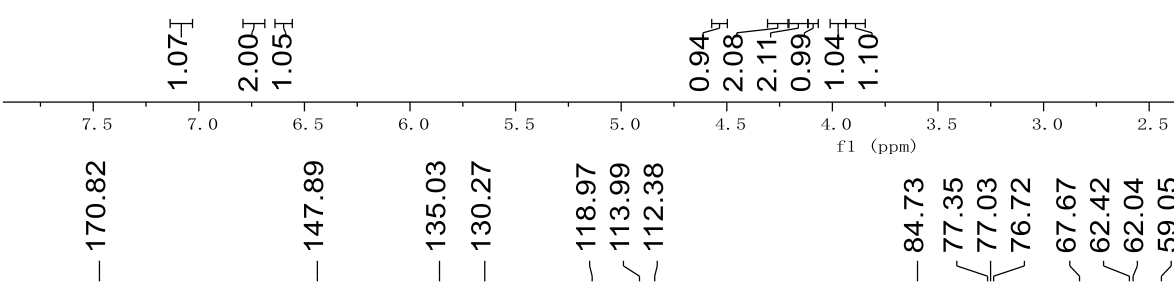
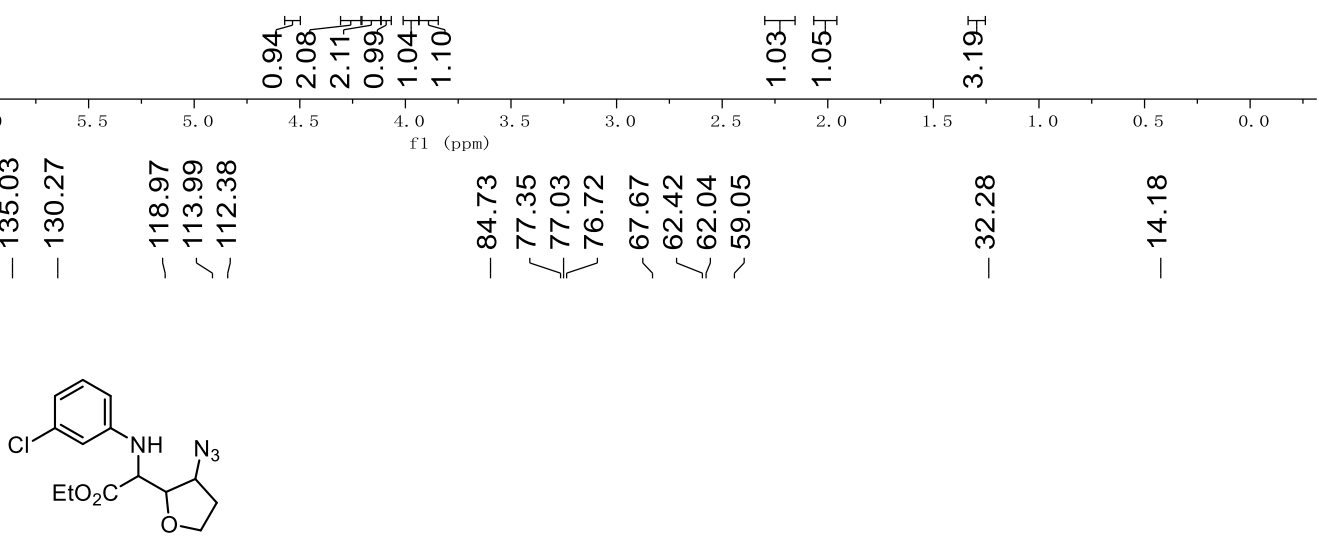

4ha, Isomer 1 (less polar) $\left(\mathrm{CDCl}_{3}, 101 \mathrm{MHz}\right)$

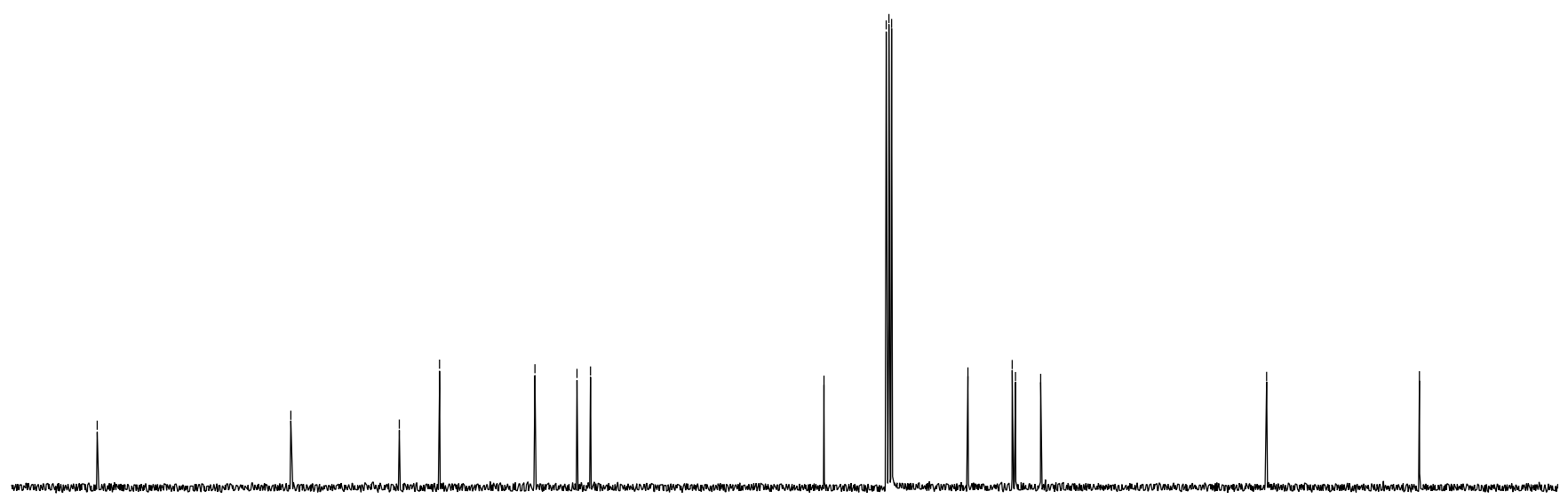

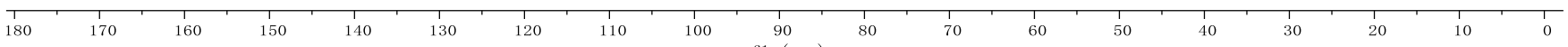




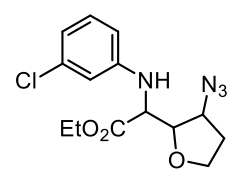

4ha, Isomer 2 (more polar) $\left(\mathrm{CDCl}_{3}, 400 \mathrm{MHz}\right)$

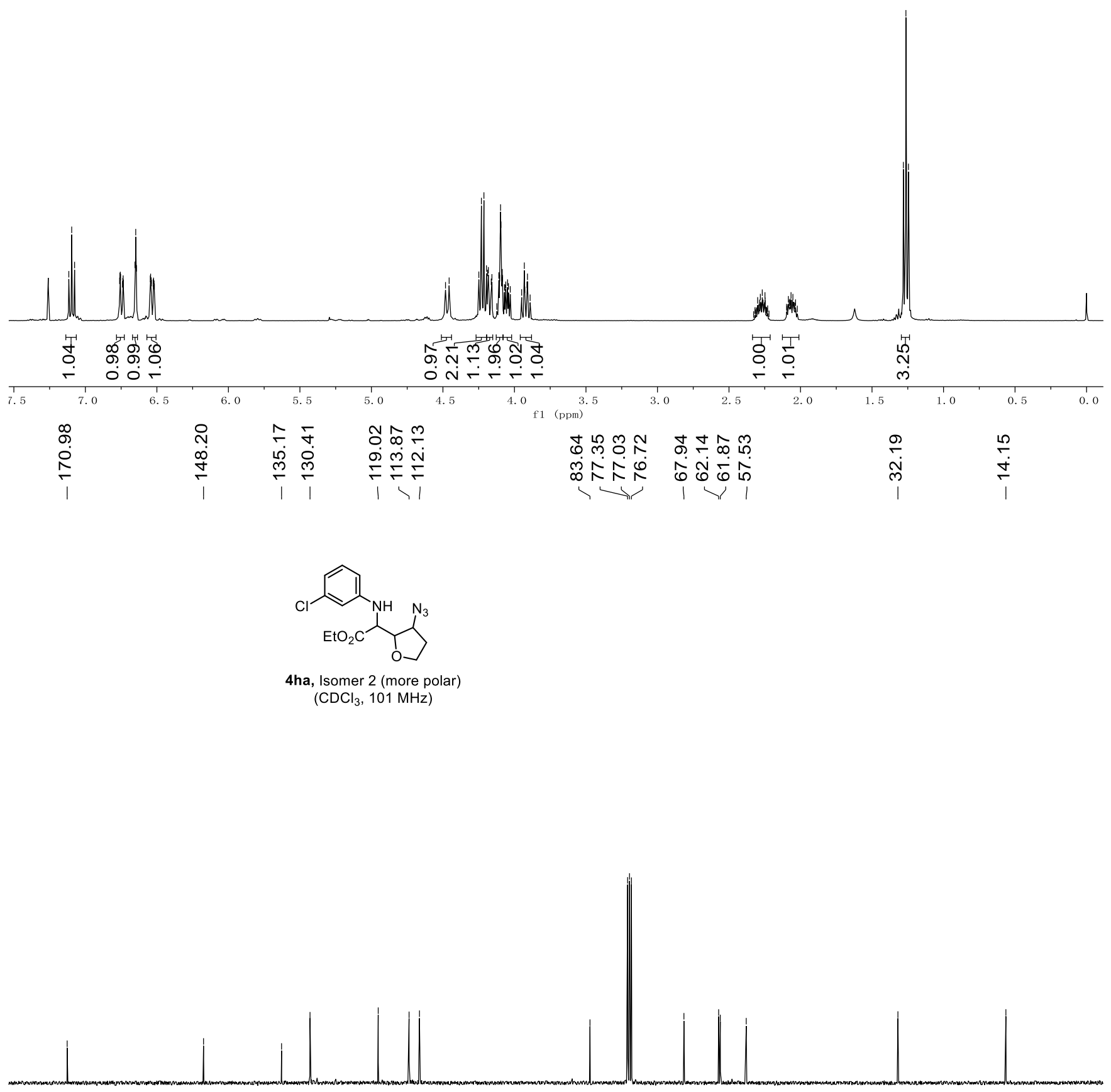

$\underset{180}{180}$

$170 \quad 160$

150

140

90
$\mathrm{f} 1$

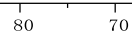

60

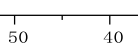




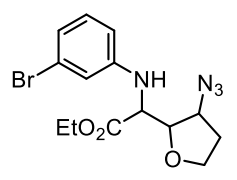

4ia, Isomer 1 (less polar) $\left(\mathrm{CDCl}_{3}, 400 \mathrm{MHz}\right)$

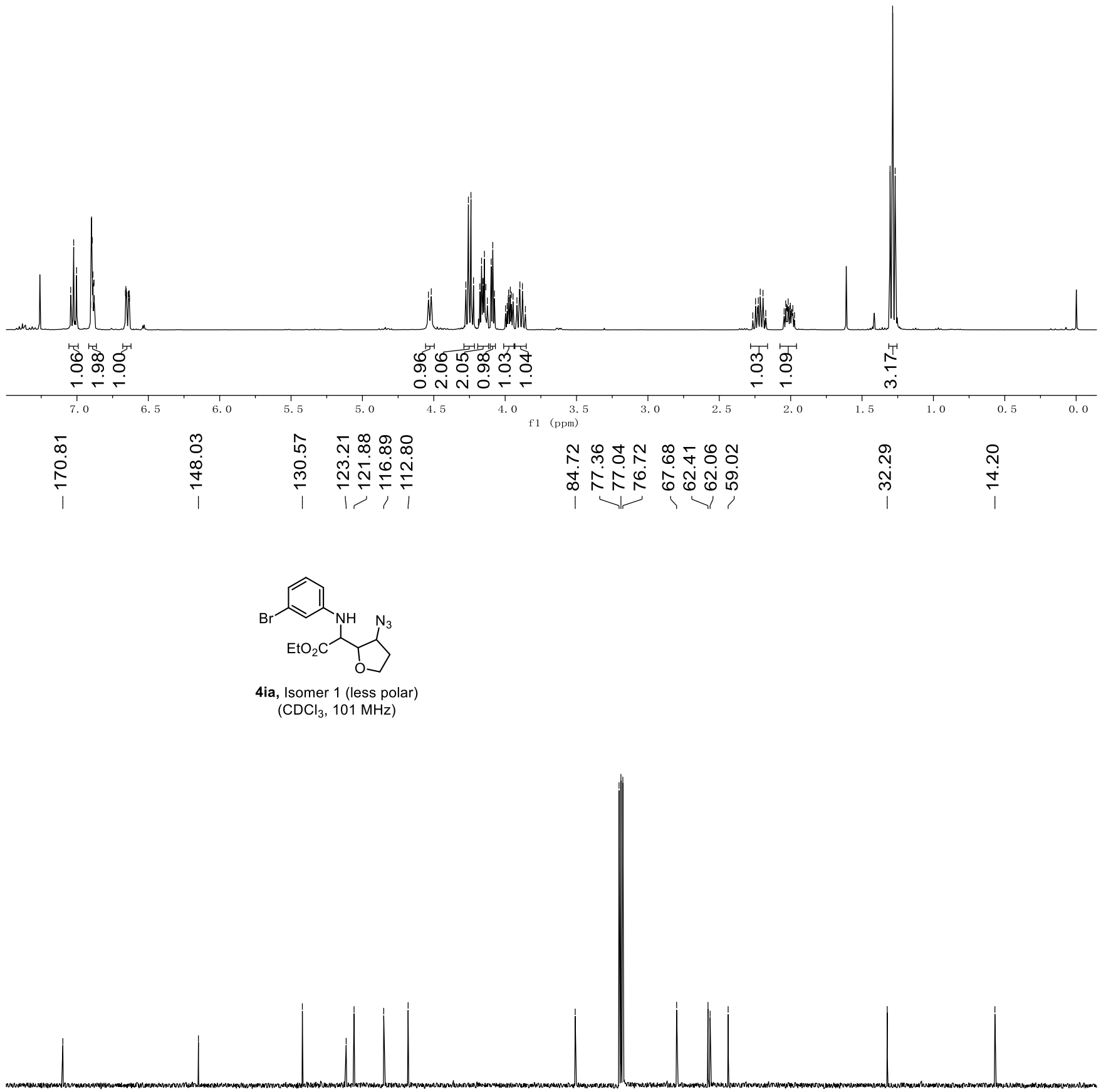

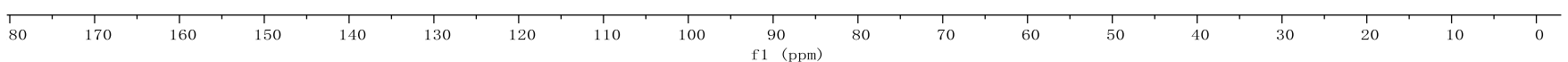




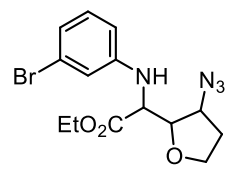

4ia, Isomer 2 (more polar) $\left(\mathrm{CDCl}_{3}, 400 \mathrm{MHz}\right)$

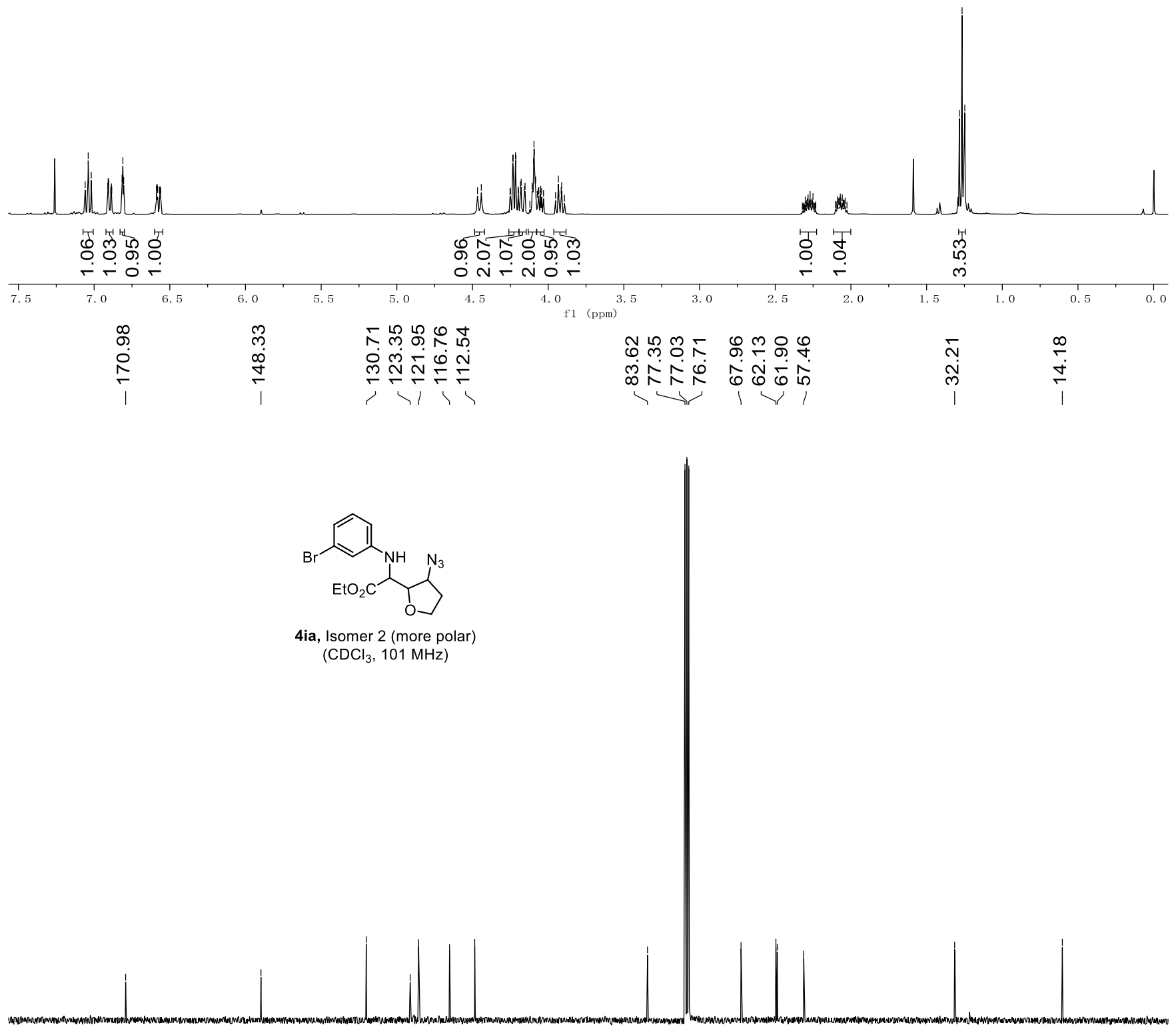

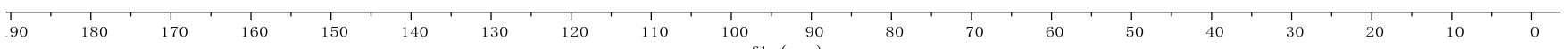




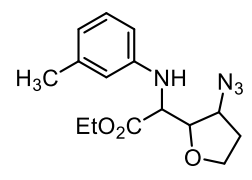

4ja, Isomer 1 (less polar)

$\left(\mathrm{CDCl}_{3}, 400 \mathrm{MHz}\right)$

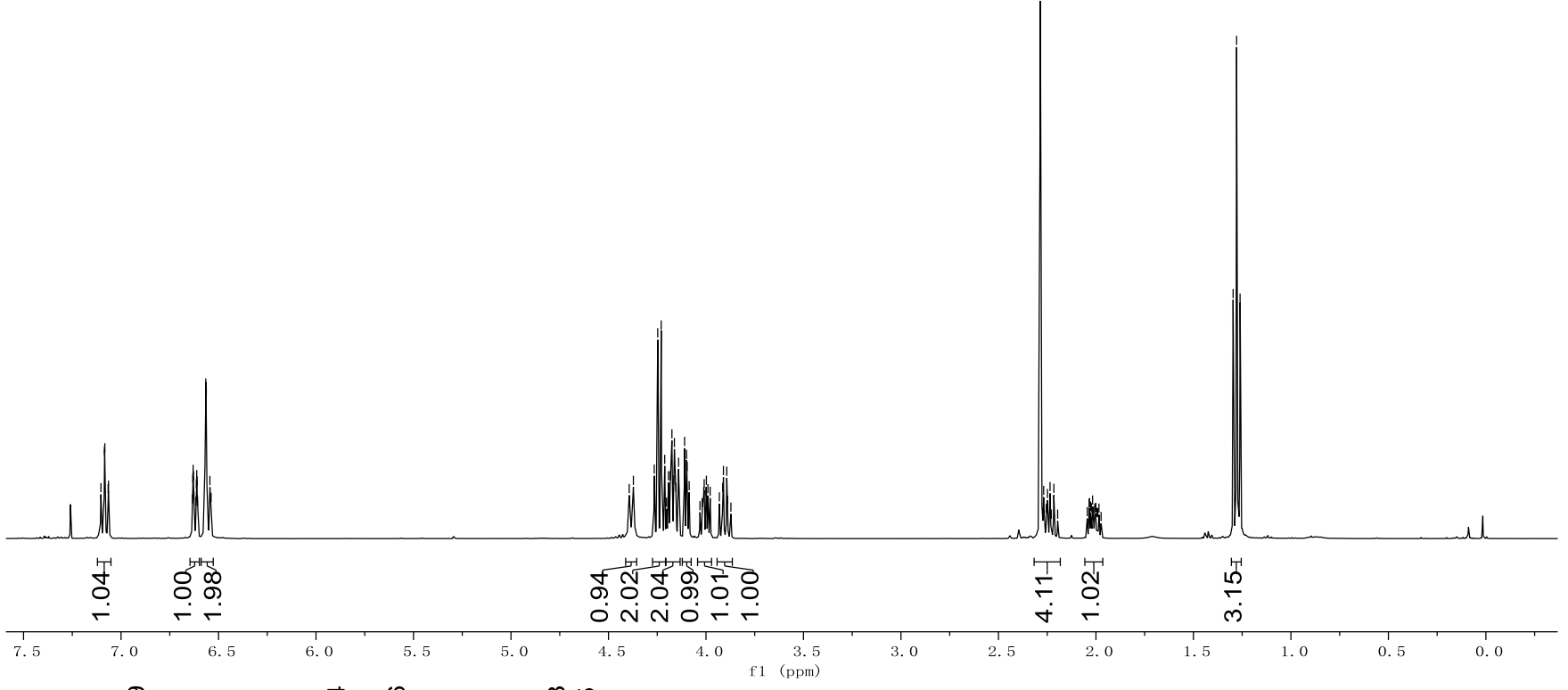

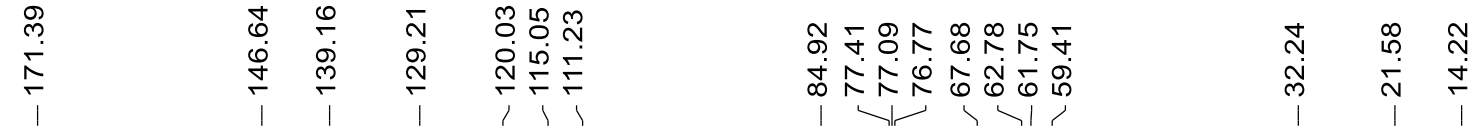
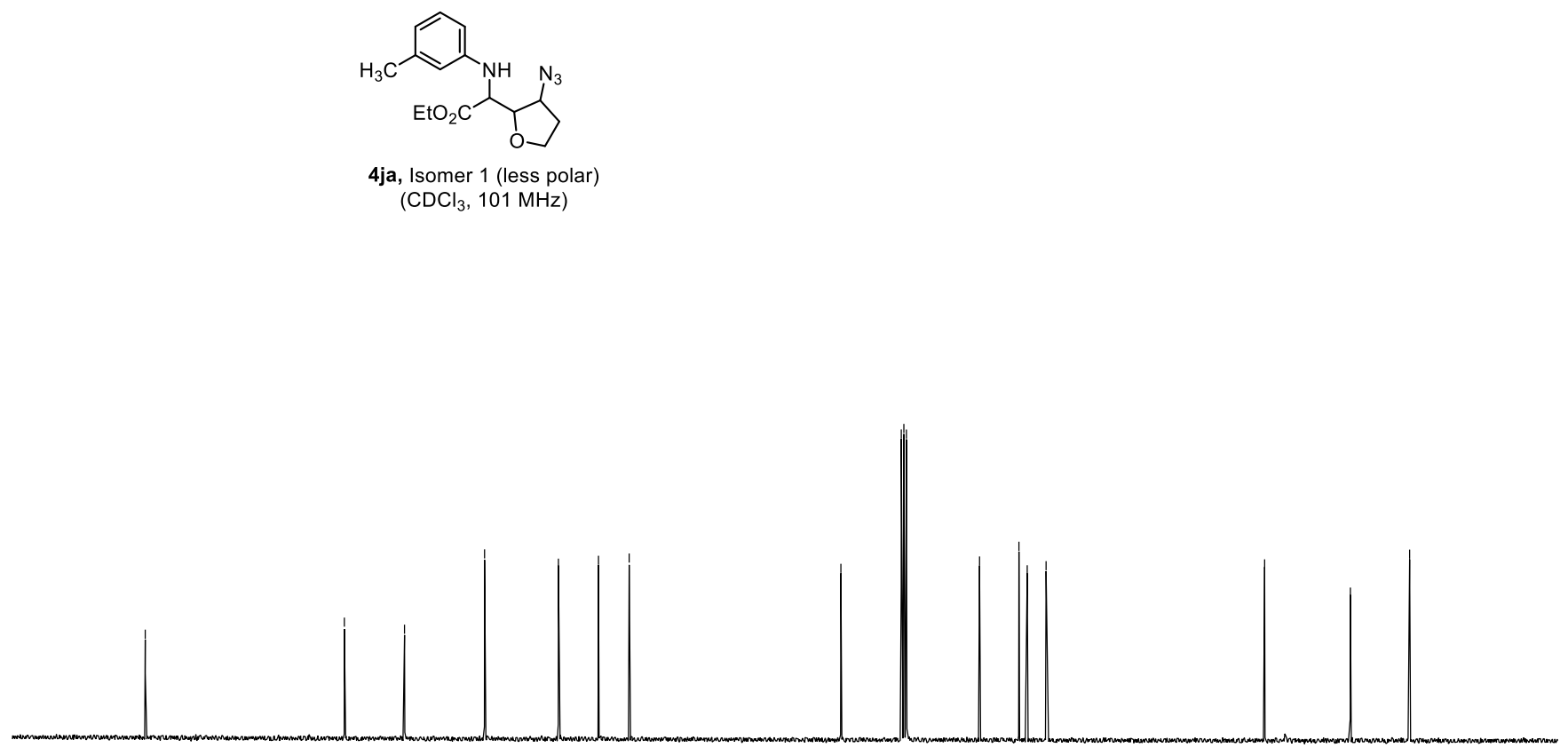

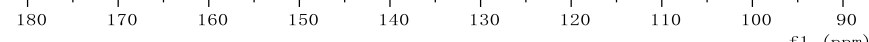




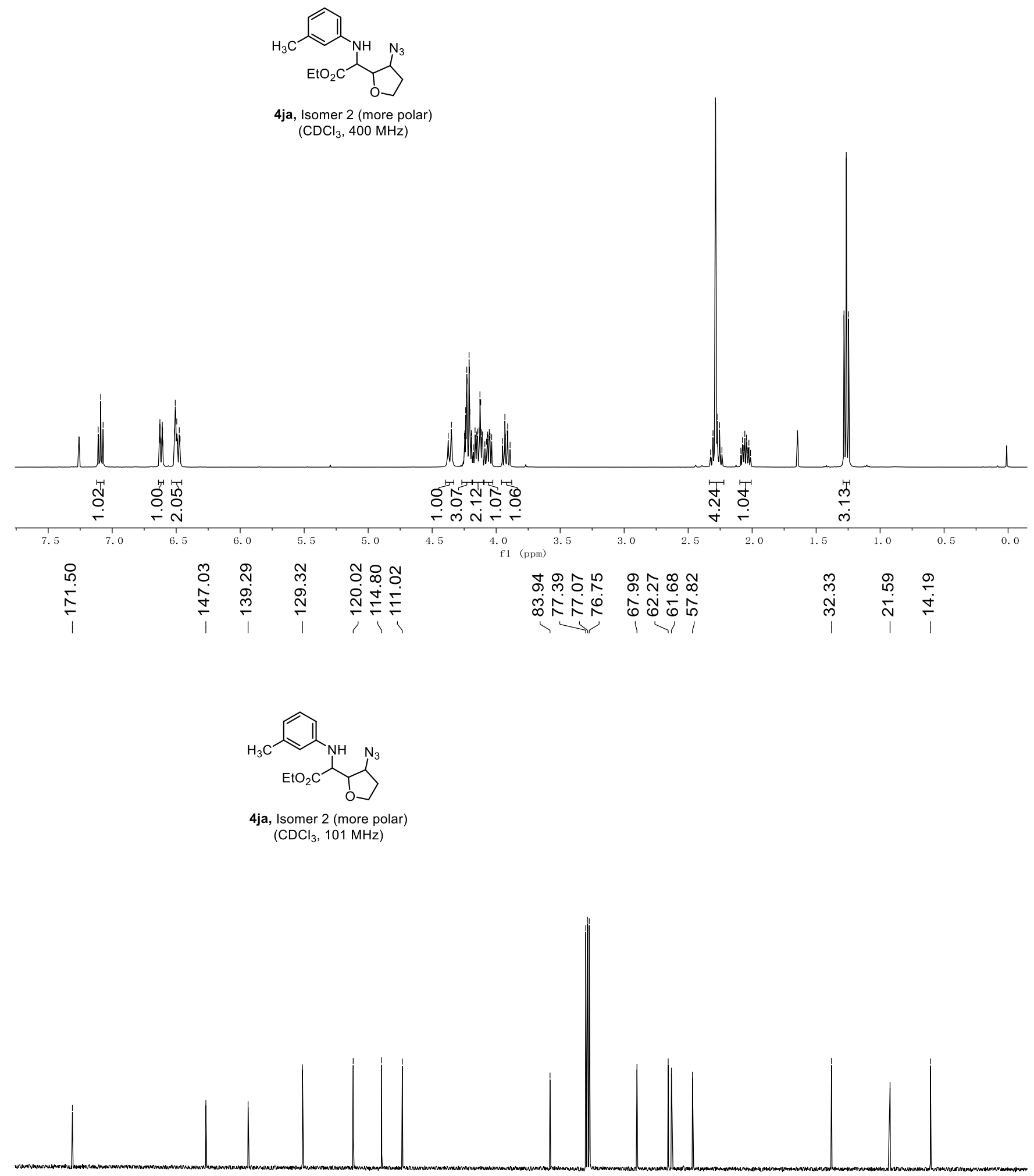



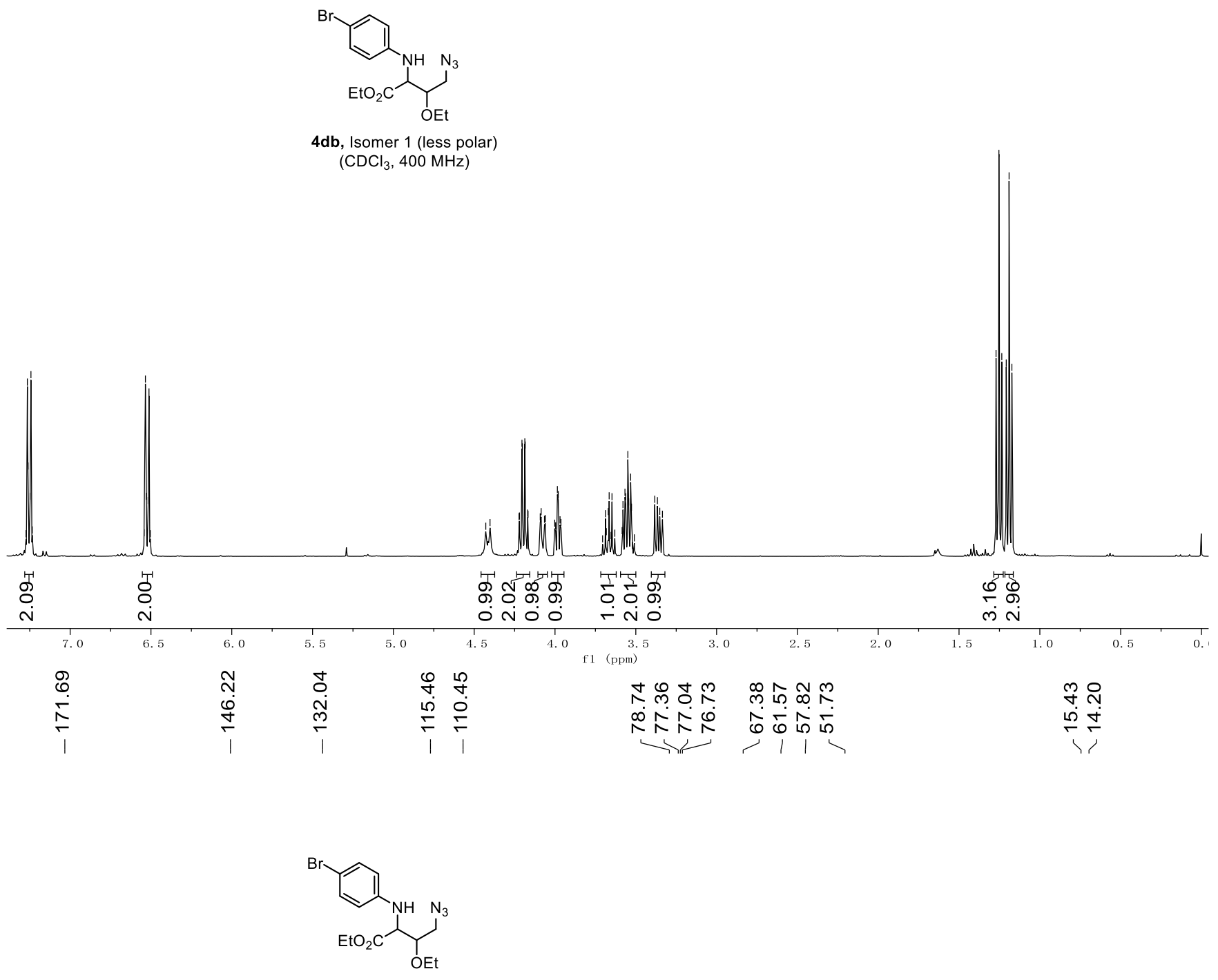

4db, Isomer 1 (less polar) $\left(\mathrm{CDCl}_{3}, 101 \mathrm{MHz}\right)$

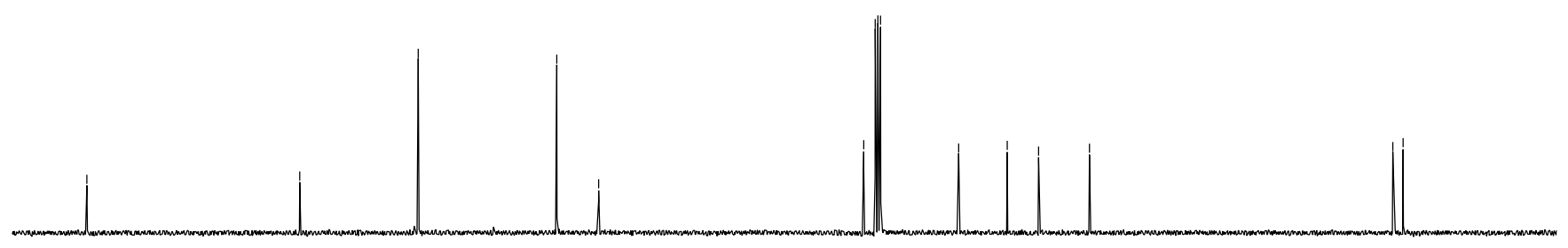

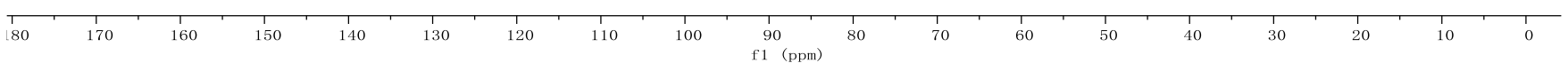




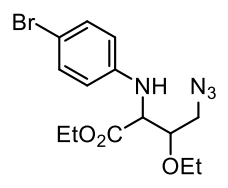

$\mathbf{4 d b}$, Isomer 2 (more polar) $\left(\mathrm{CDCl}_{3}, 400 \mathrm{MHz}\right)$
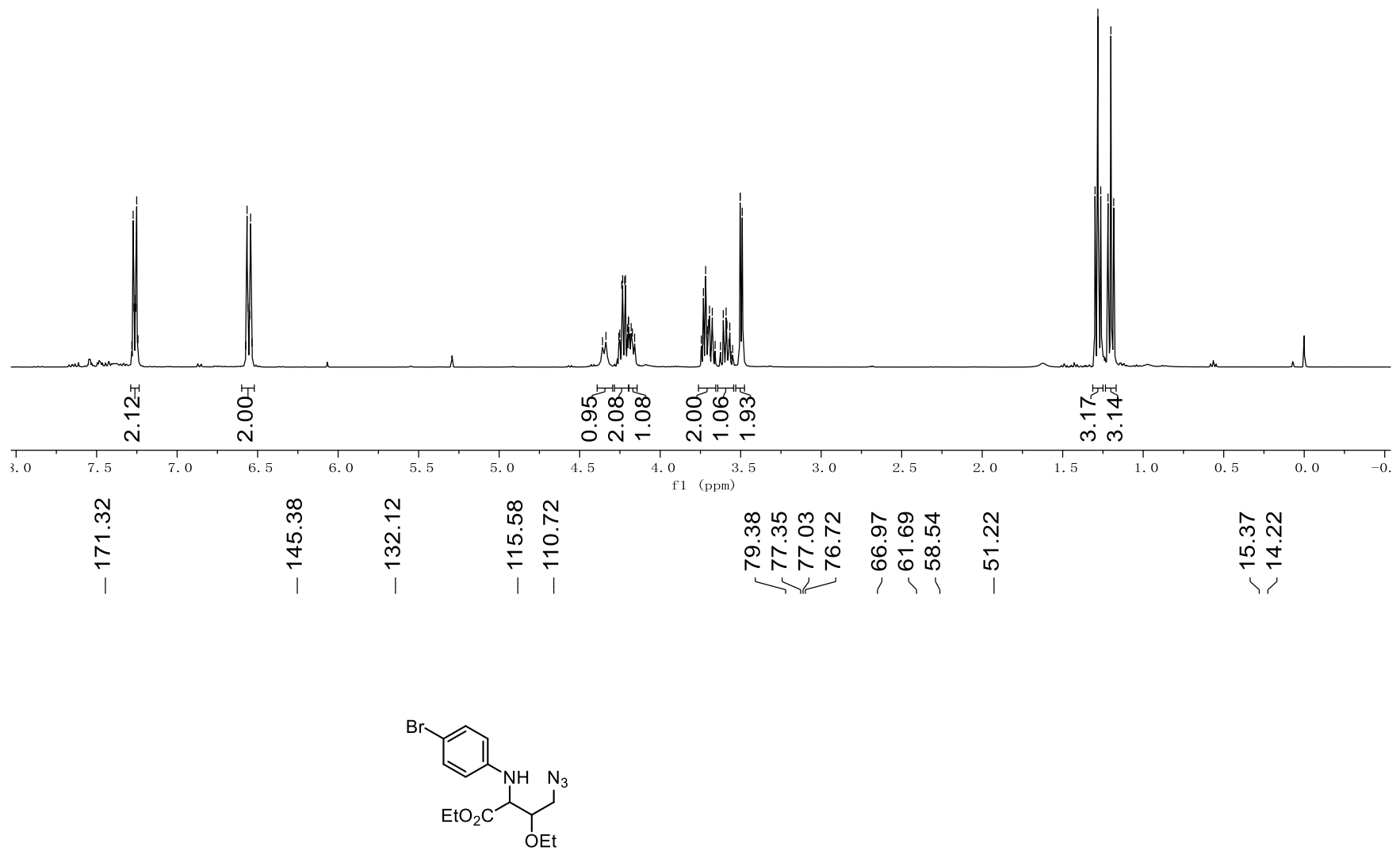

4db, Isomer 2 (more polar) $\left(\mathrm{CDCl}_{3}, 101 \mathrm{MHz}\right)$

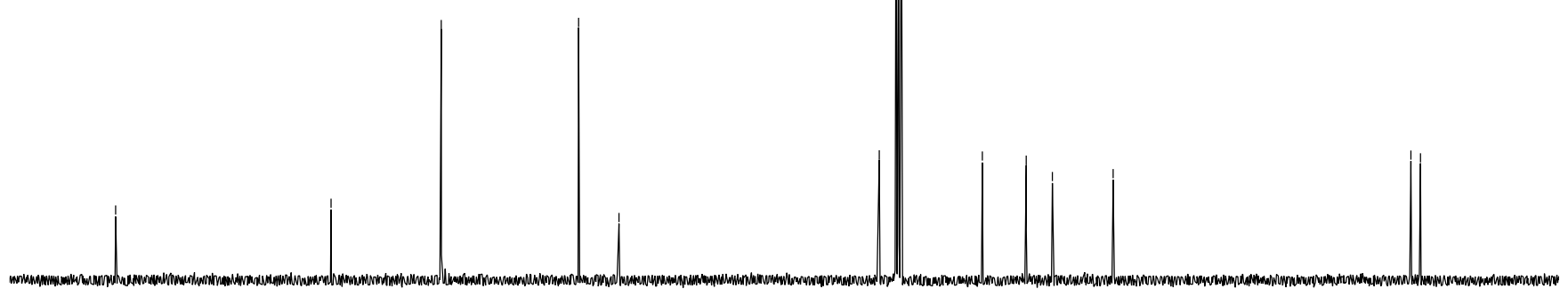

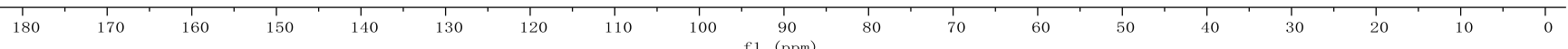




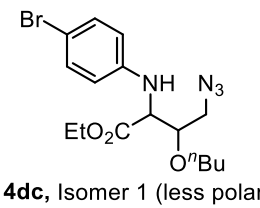

$\left(\mathrm{CDCl}_{3}, 600 \mathrm{MHz}\right)$
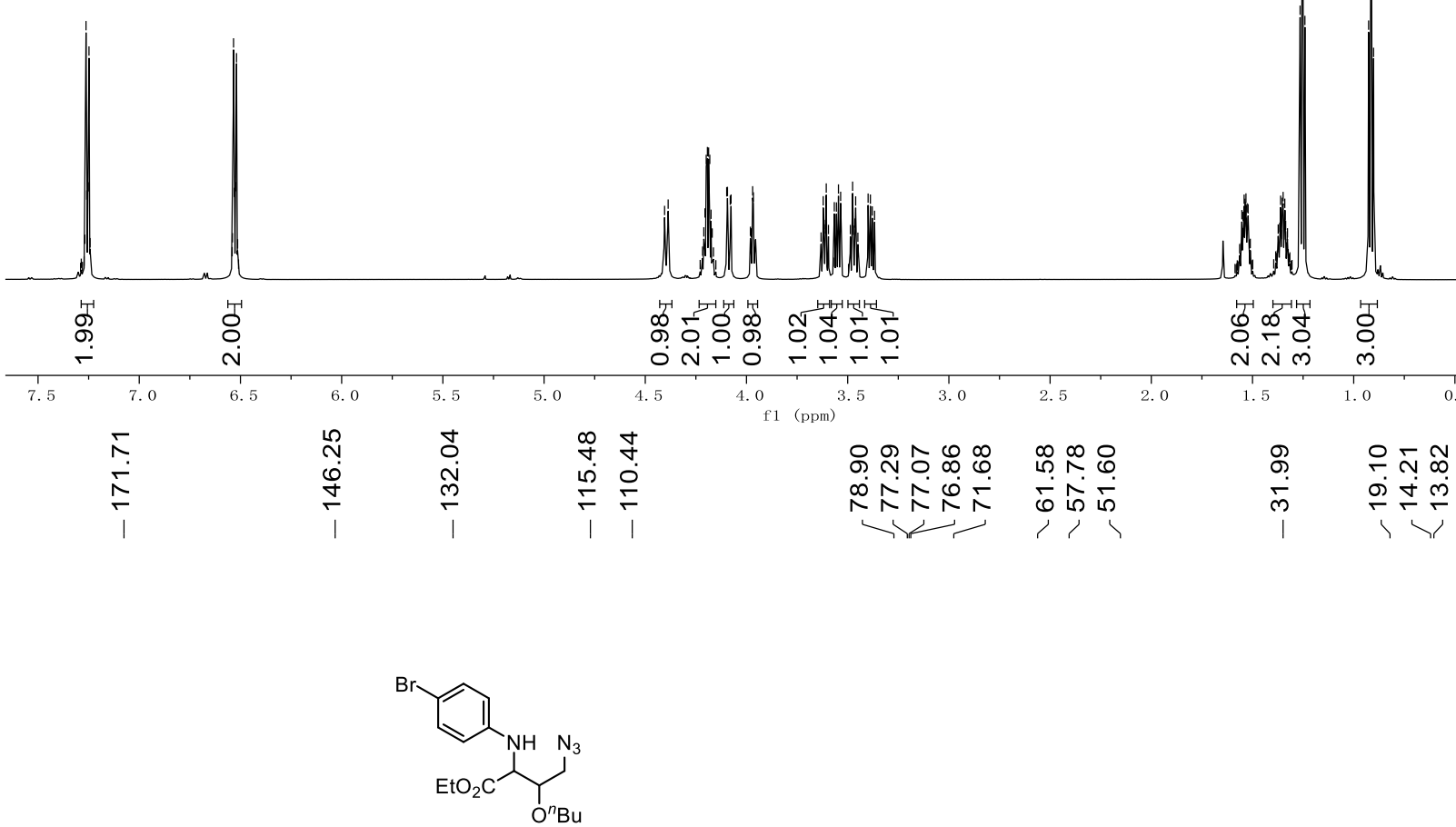

4dc, Isomer 1 (less polar) $\left(\mathrm{CDCl}_{3}, 151 \mathrm{MHz}\right)$

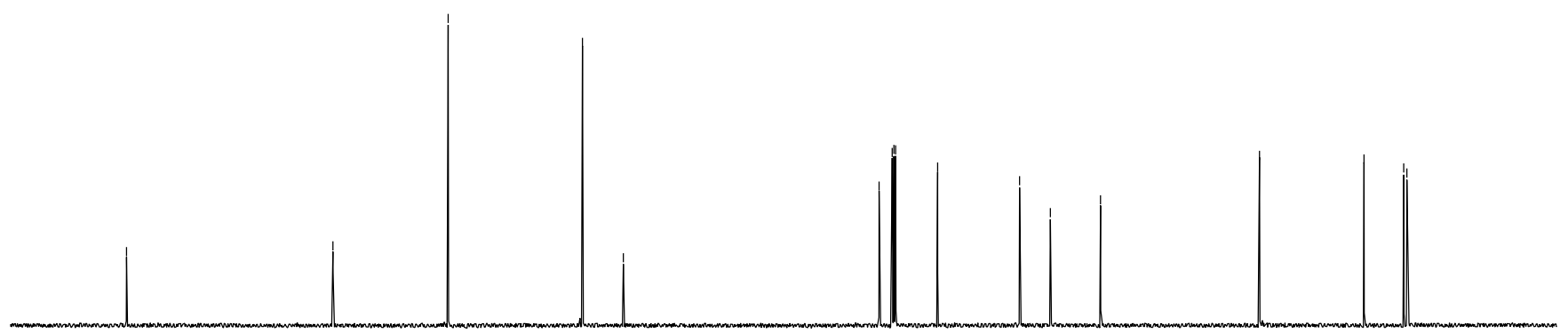

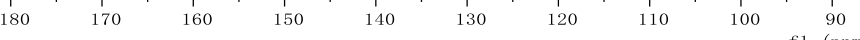



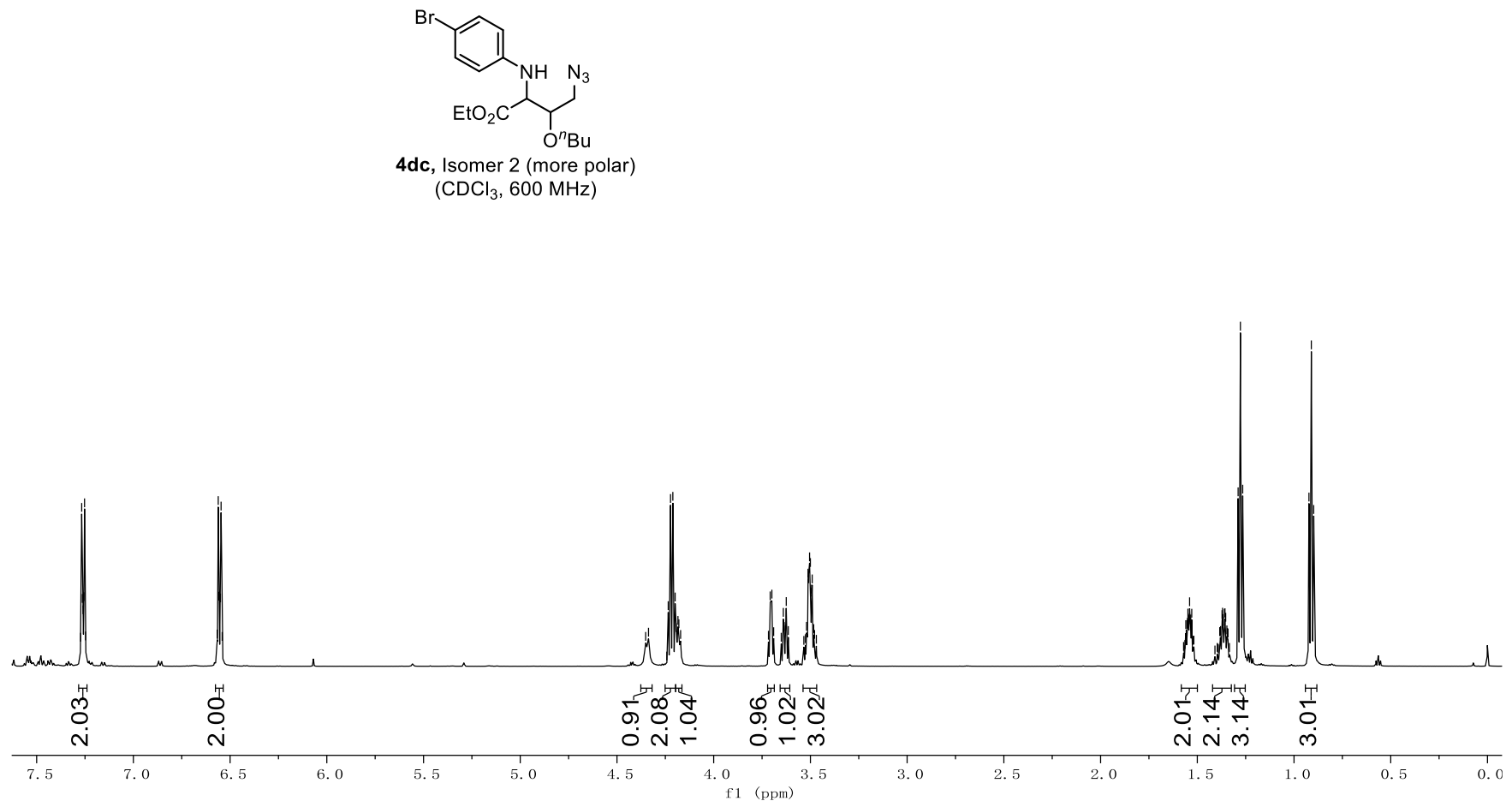

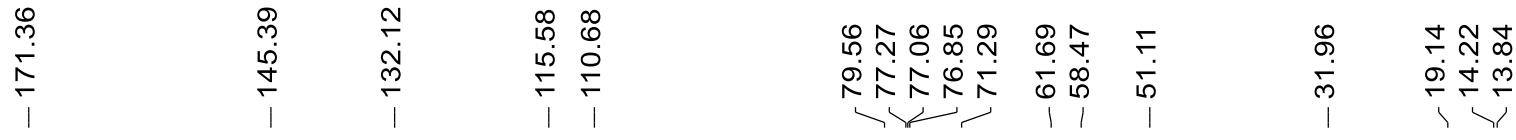

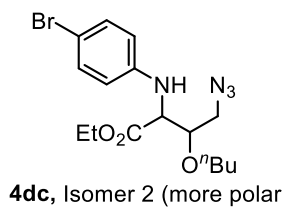

$$
\left(\mathrm{CDCl}_{3}, 151 \mathrm{MHz}\right)
$$

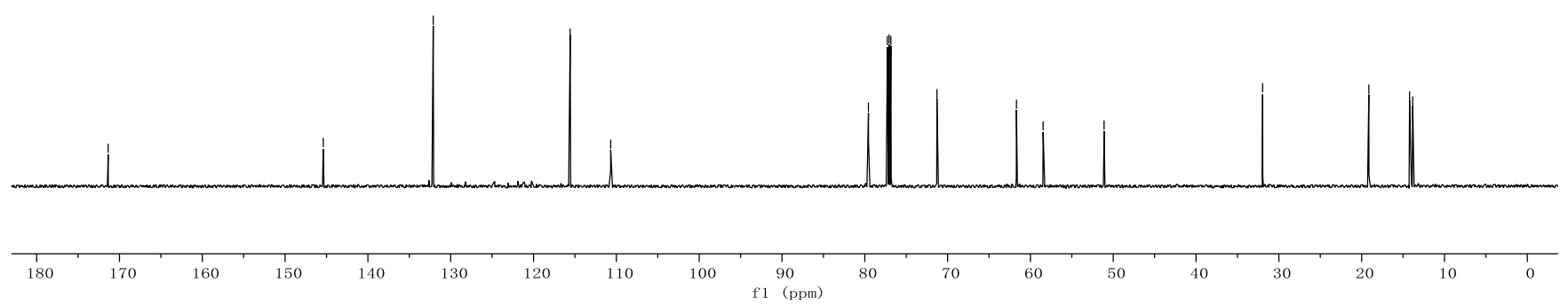




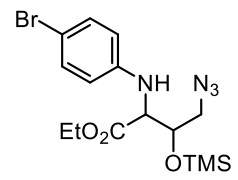

4dd, Isomer 1 (less polar)

$\left(\mathrm{CDCl}_{3}, 600 \mathrm{MHz}\right)$

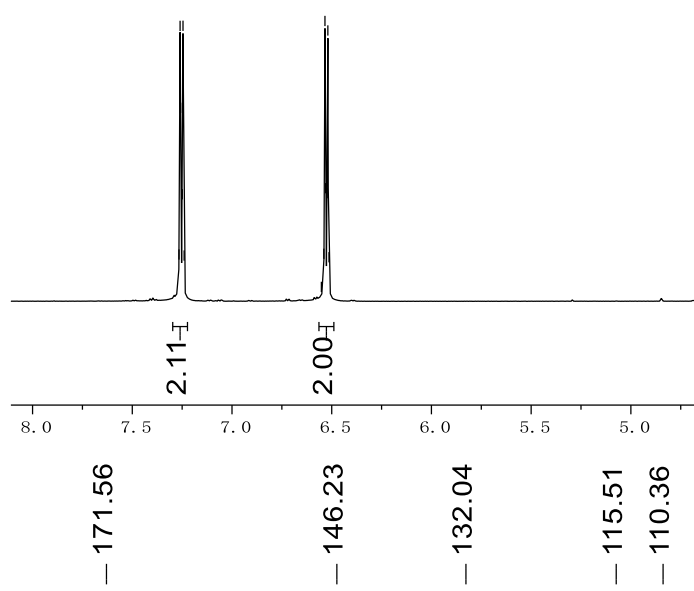

(H)

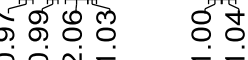

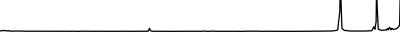

hul
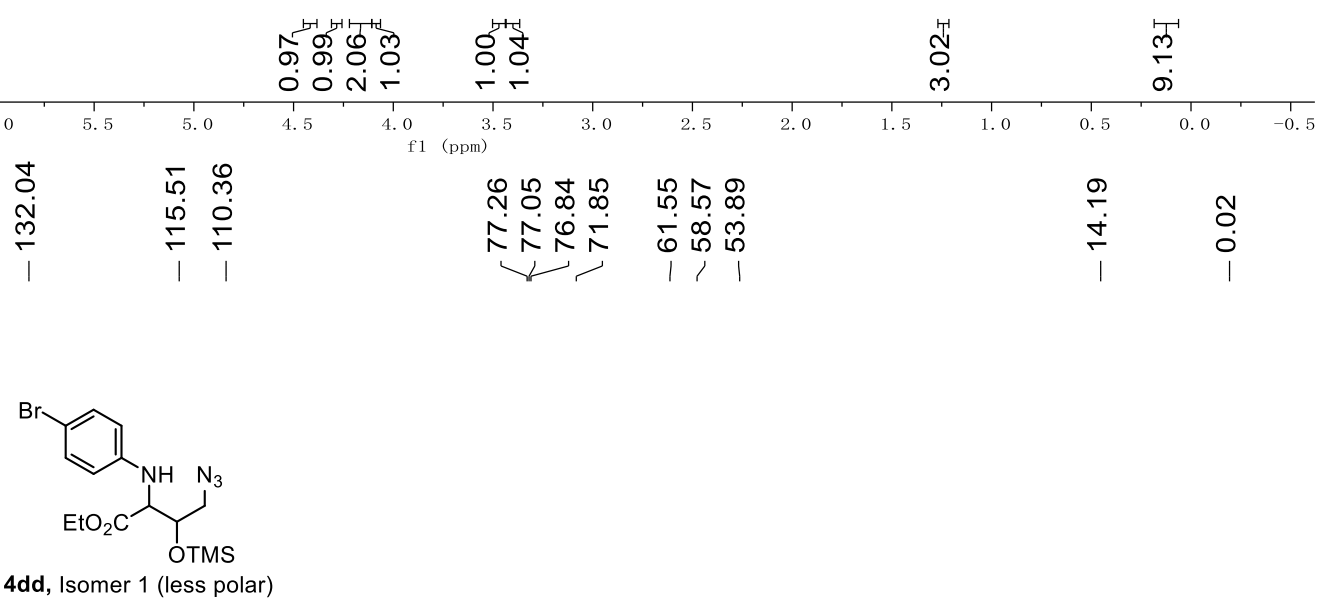

$\left(\mathrm{CDCl}_{3}, 151 \mathrm{MHz}\right)$

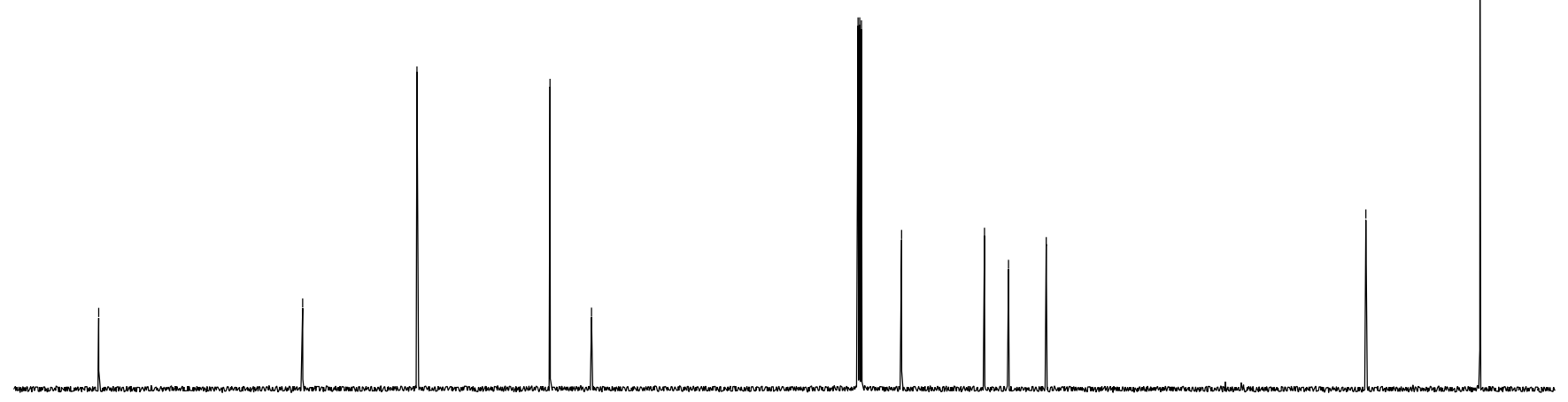

$\frac{1}{180}$

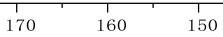

140

130

120

90

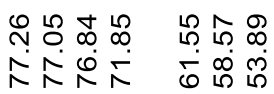

$\begin{array}{ll}\stackrel{0}{r} & 0 \\ \stackrel{1}{*} & 0 \\ 1 & 1\end{array}$ 

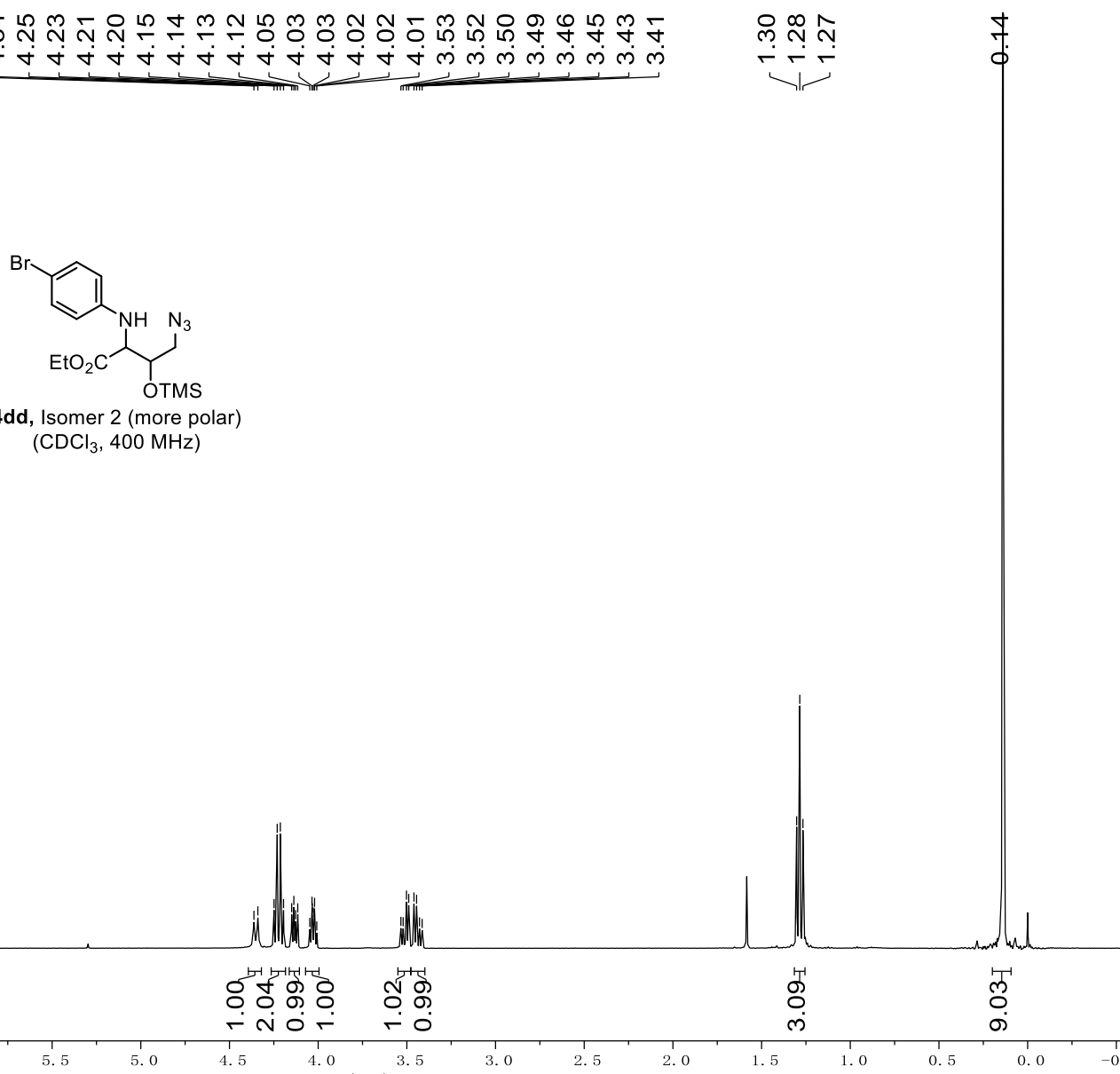

4dd, Isomer 2 (more polar) $\left(\mathrm{CDCl}_{3}, 400 \mathrm{MHz}\right)$
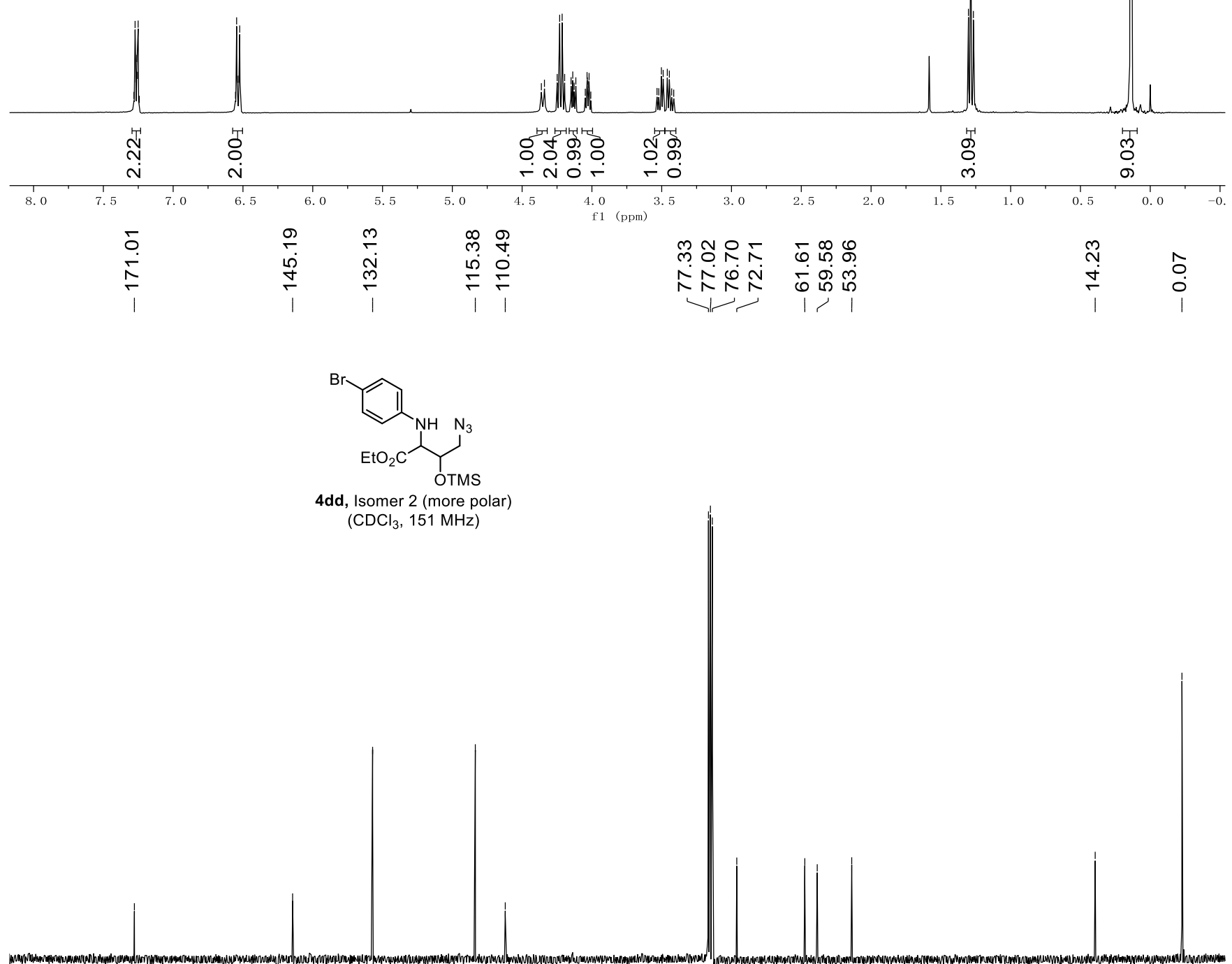

4dd, Isomer 2 (more polar) $\left(\mathrm{CDCl}_{3}, 151 \mathrm{MHz}\right)$

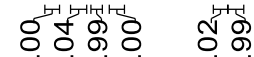
- No- 4. 0 (ppm)

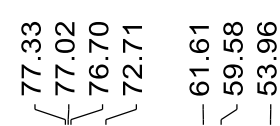




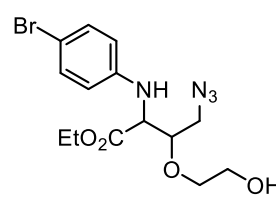

4de, Isomer $1+$ Isomer 2

$\left(\mathrm{CDCl}_{3}, 600 \mathrm{MHz}\right)$
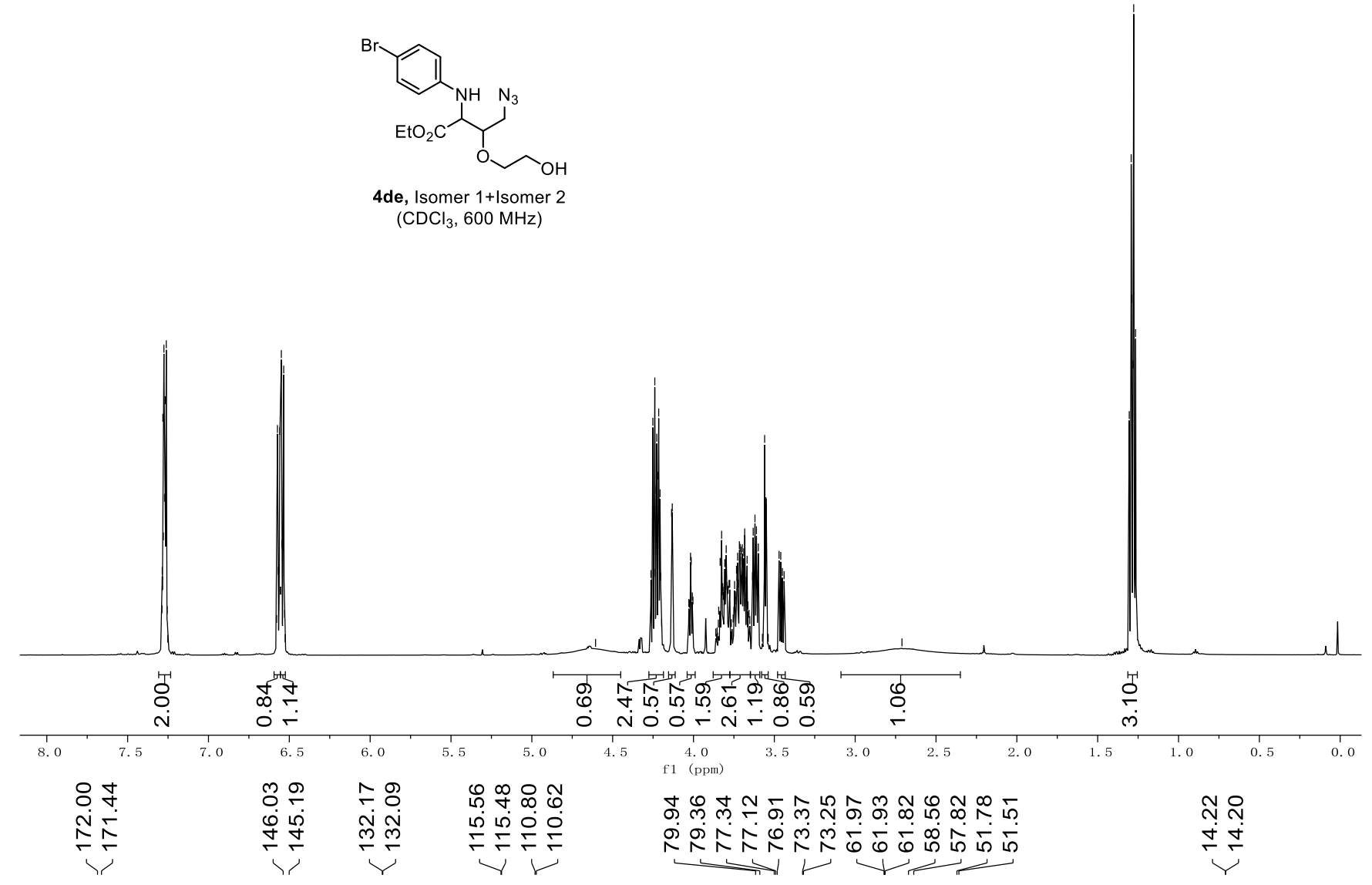

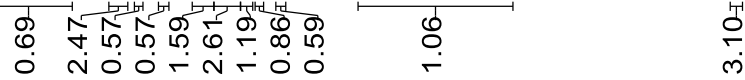

ل

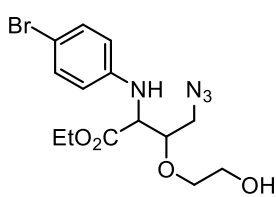

4de, Isomer $1+$ Isomer 2 $\left(\mathrm{CDCl}_{3}, 151 \mathrm{MHz}\right)$

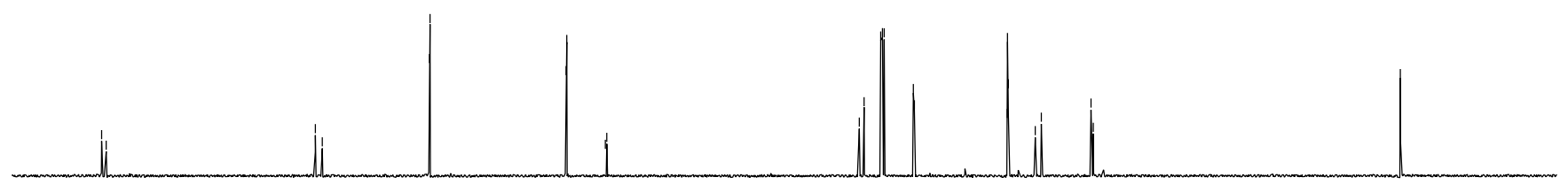




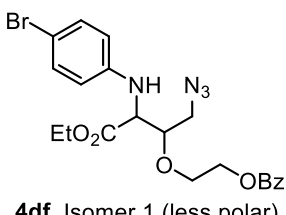

4df, Isomer 1 (less polar)

$\left(\mathrm{CDCl}_{3}, 400 \mathrm{MHz}\right)$
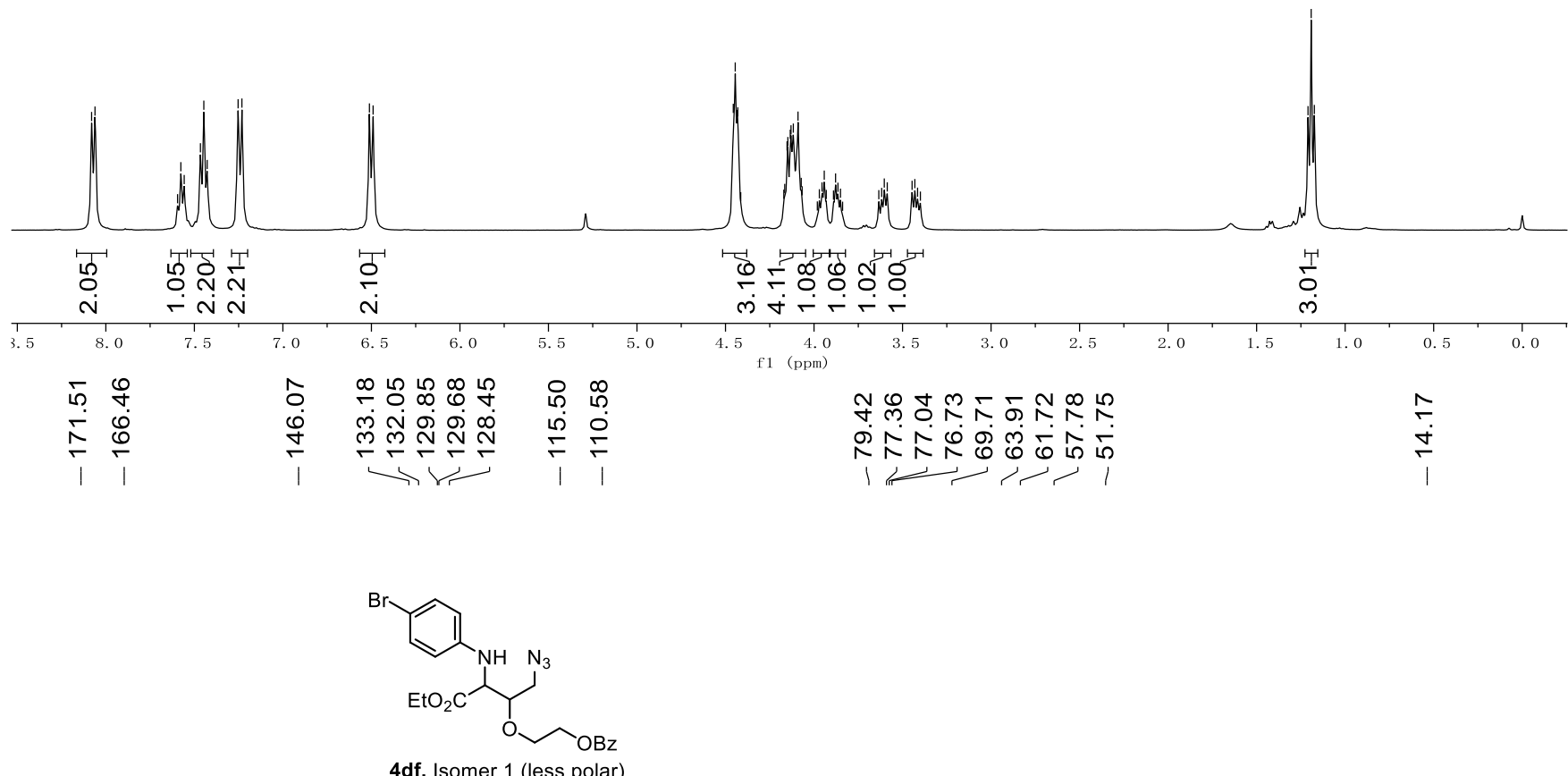

4df, Isomer 1 (less polar)

$\left(\mathrm{CDCl}_{3}, 101 \mathrm{MHz}\right)$
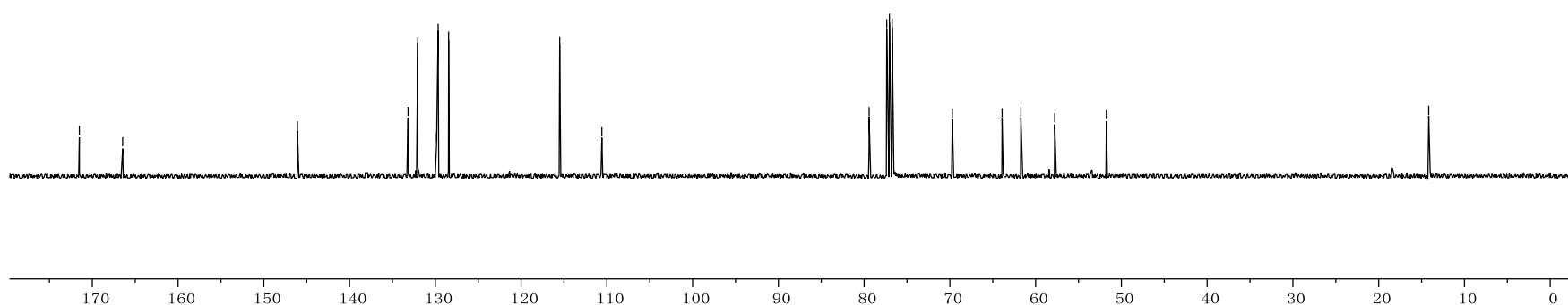

140

${ }^{90}$

$80+70,60$

50

40

30 


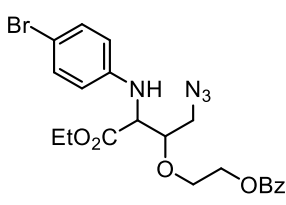

4df, Isomer 2 (more polar) $\left(\mathrm{CDCl}_{3}, 400 \mathrm{MHz}\right)$

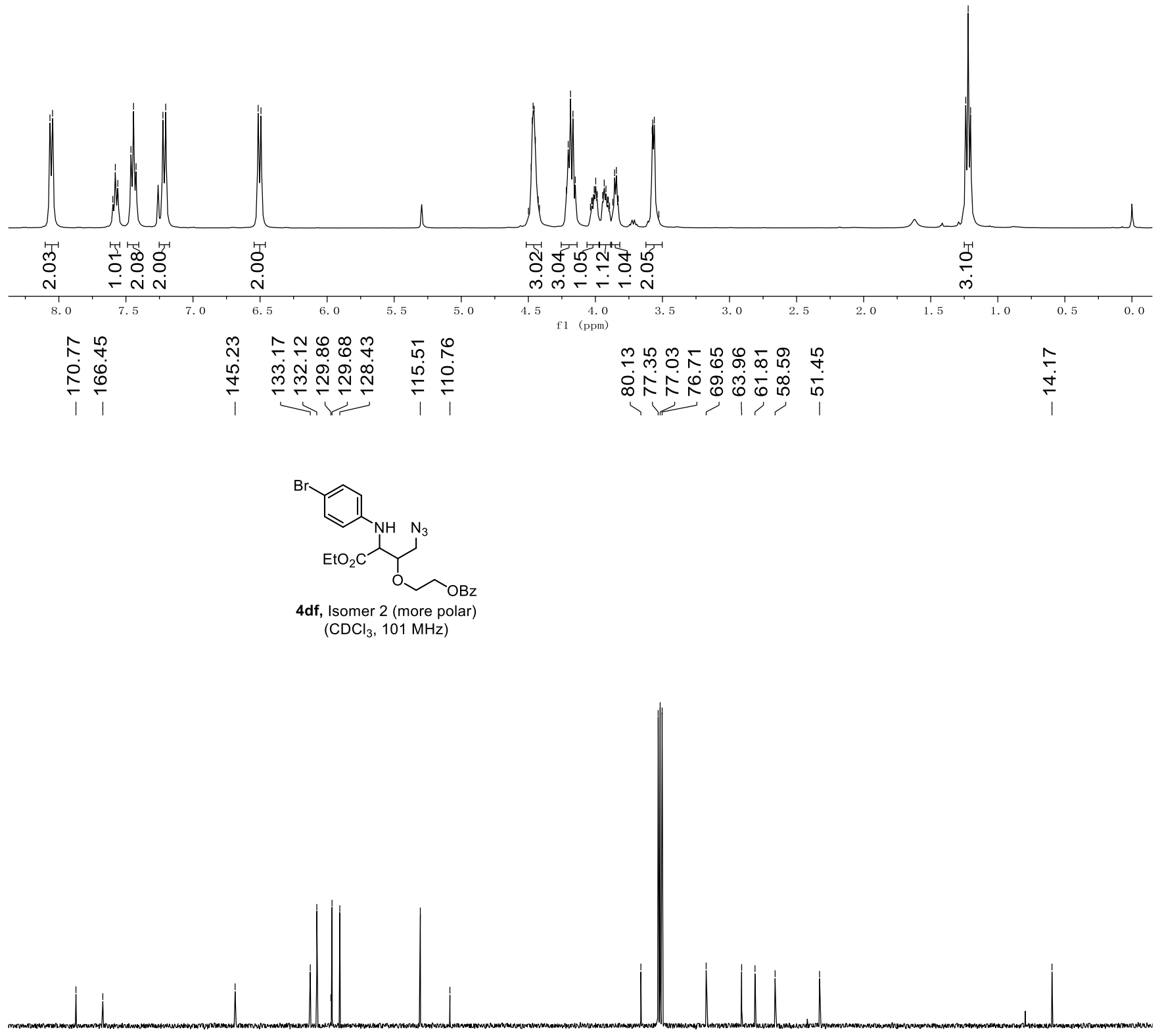

T180

$70 \quad 160$

150

f1 $\stackrel{90}{(\mathrm{ppm})}$

80

70

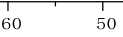

40

30 


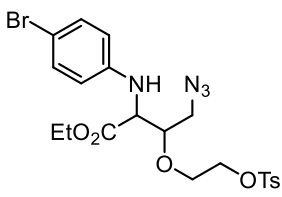

$4 \mathrm{dg}$, Isomer 1 (less polar)

$\left(\mathrm{CDCl}_{3}, 400 \mathrm{MHz}\right)$

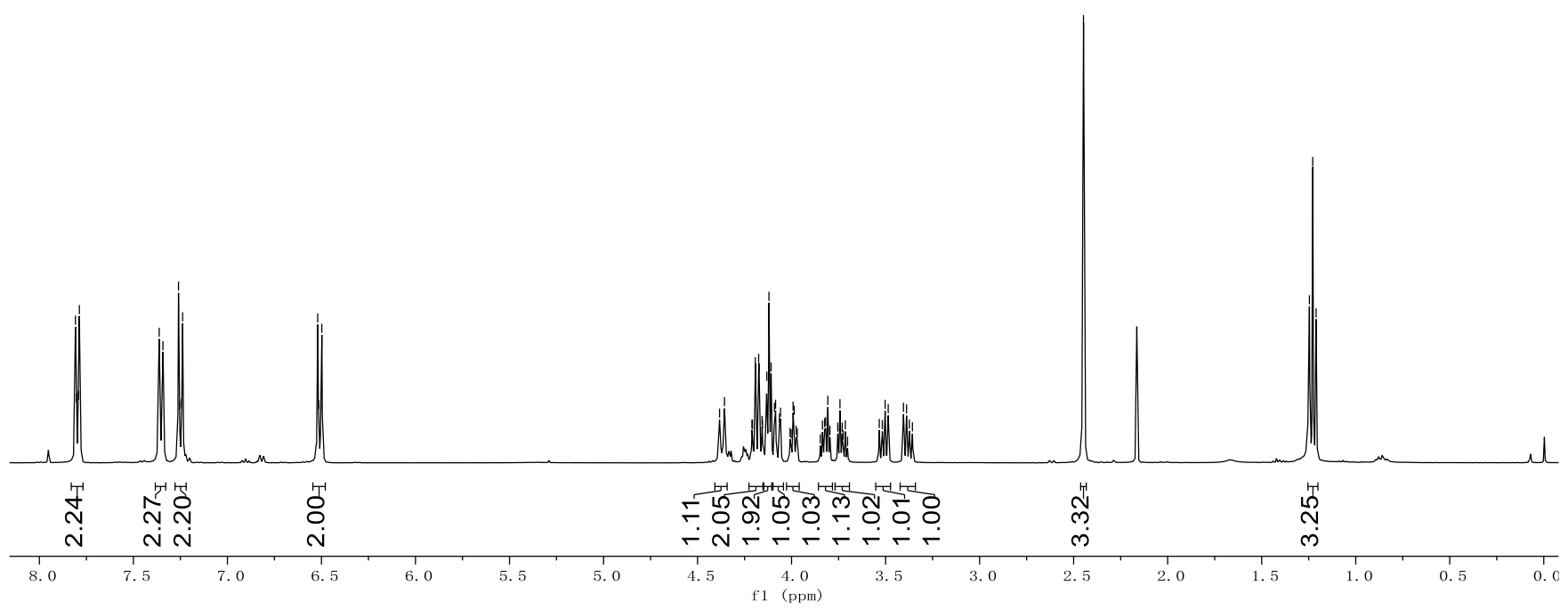

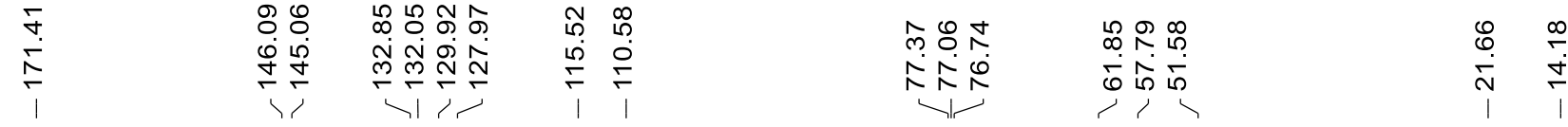

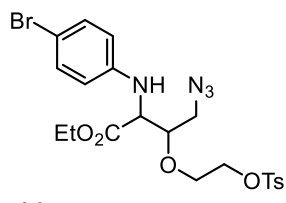

4dg, Isomer 1 (less polar)

$\left(\mathrm{CDCl}_{3}, 151 \mathrm{MHz}\right)$

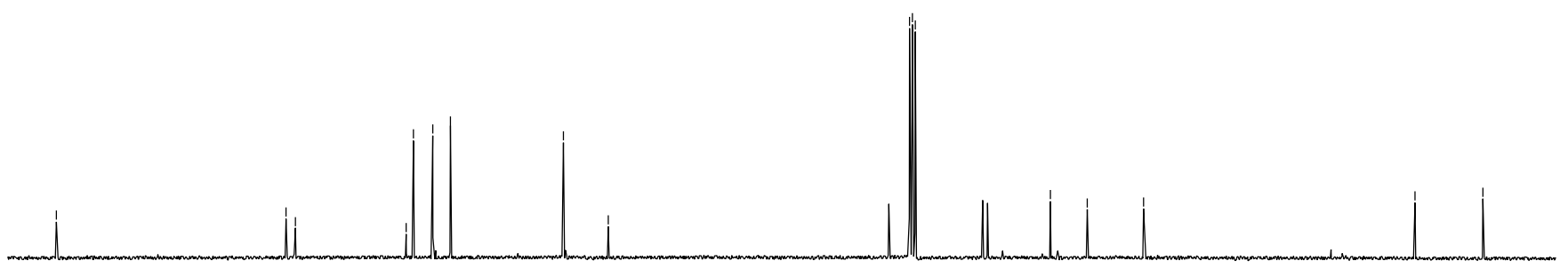

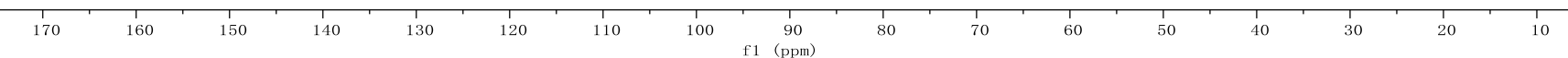




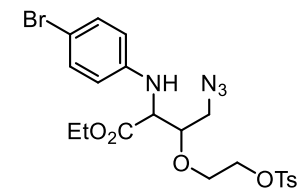

4dg, Isomer 2 (more polar) $\left(\mathrm{CDCl}_{3}, 400 \mathrm{MHz}\right)$

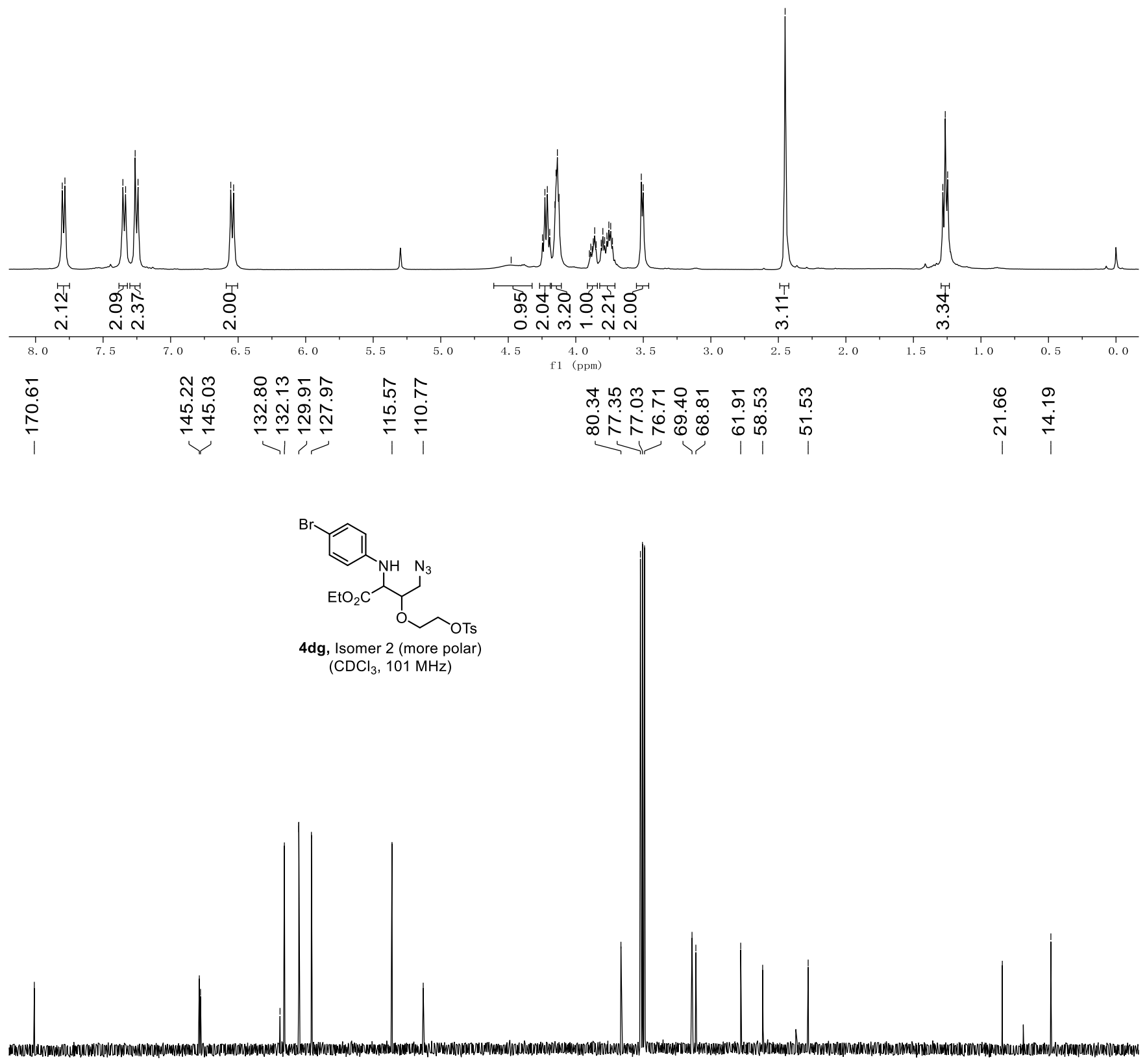

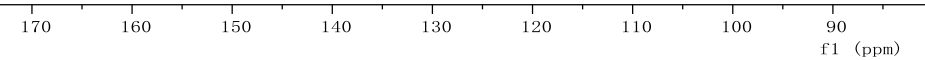



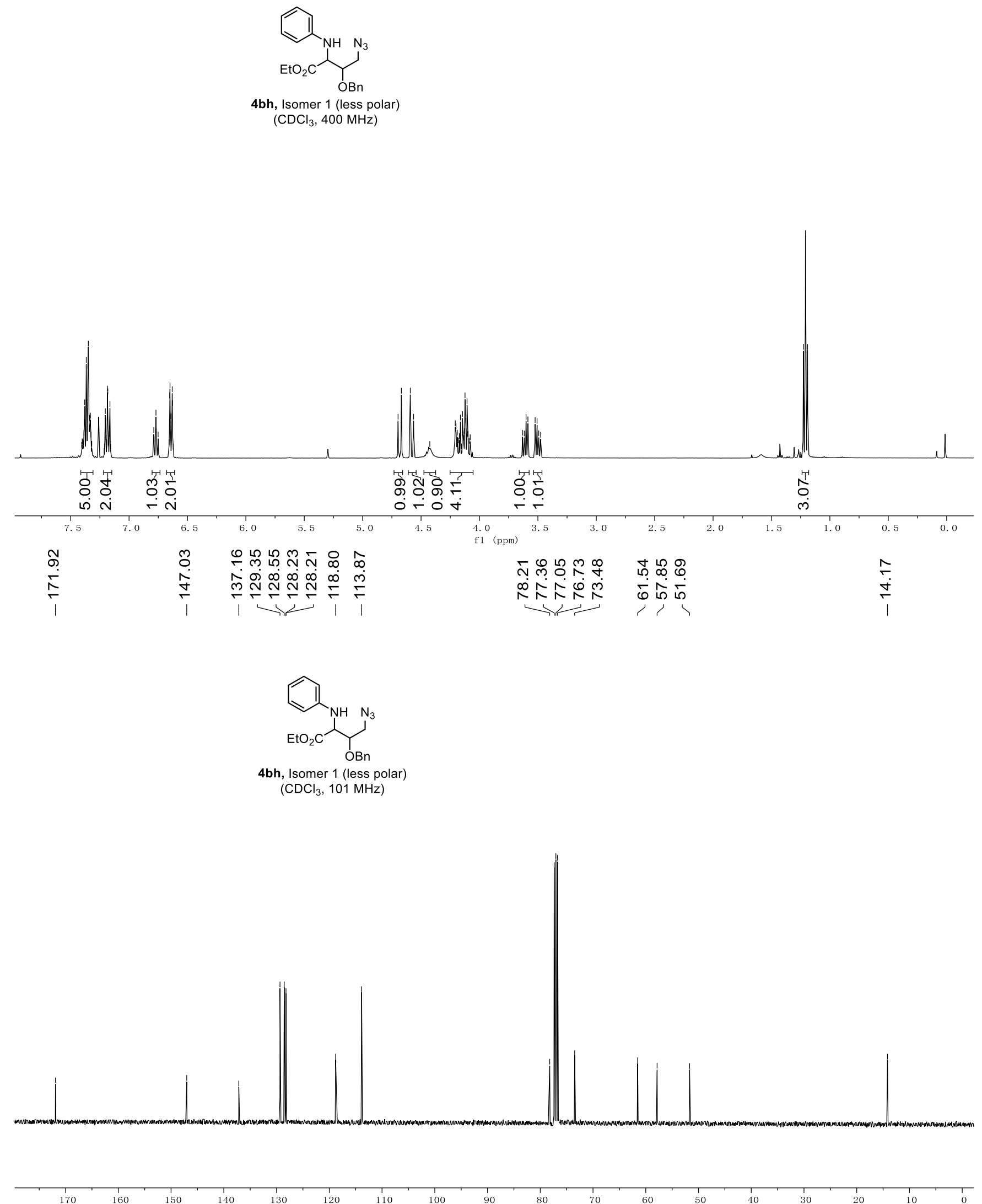

140

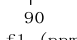

80

60

50

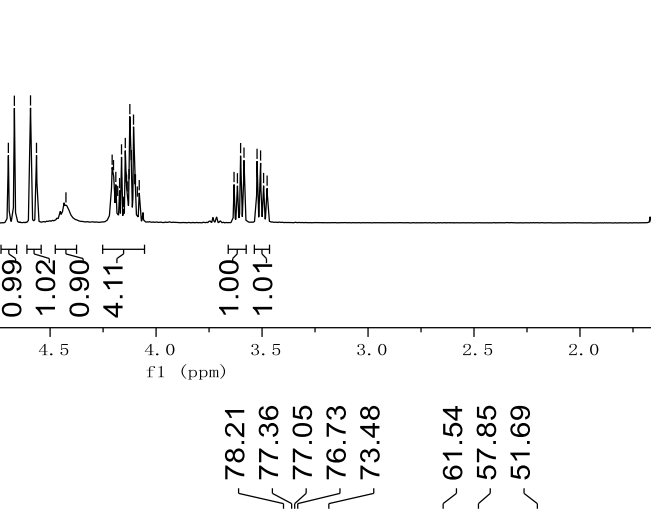



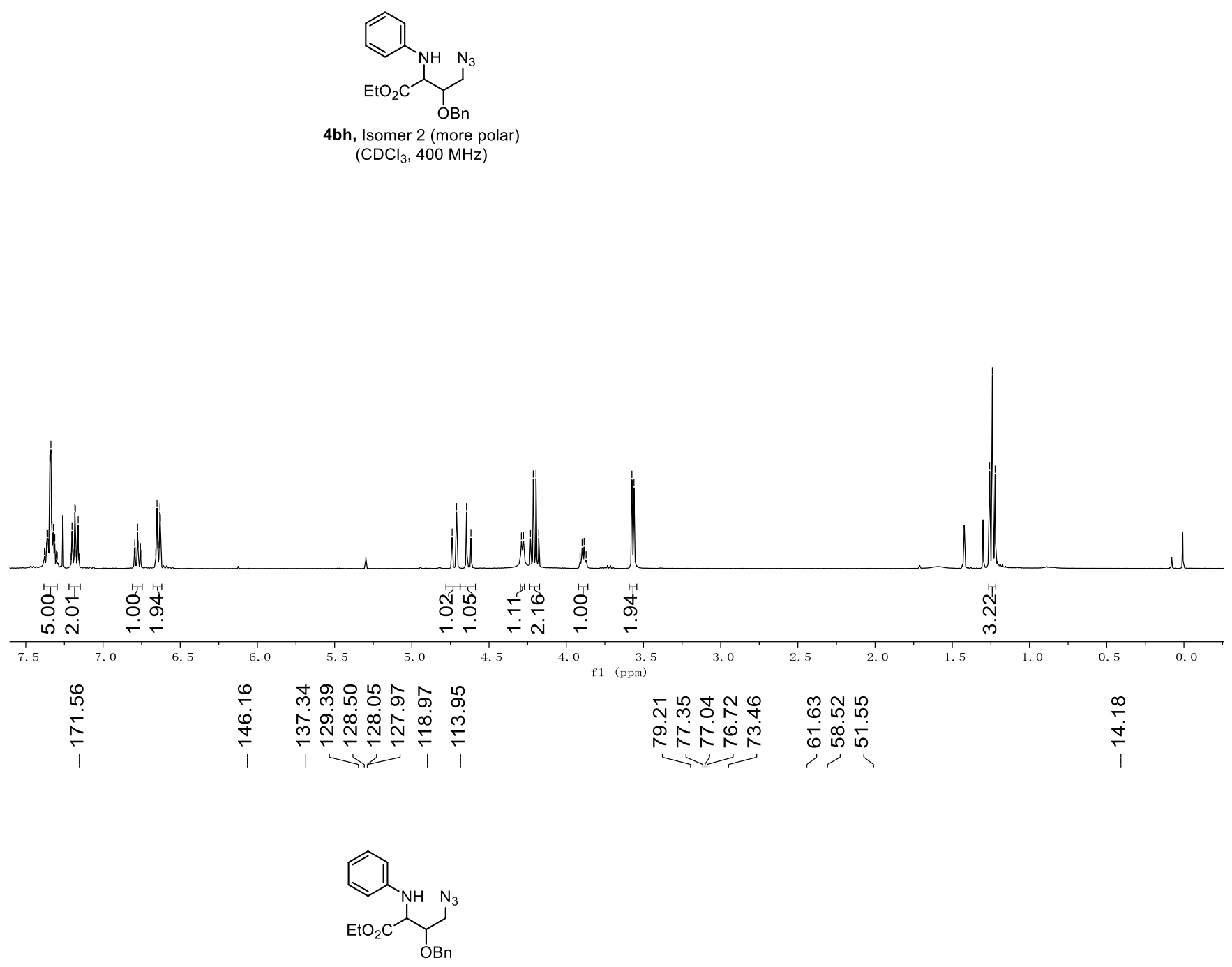

4bh, Isomer 2 (more polar)

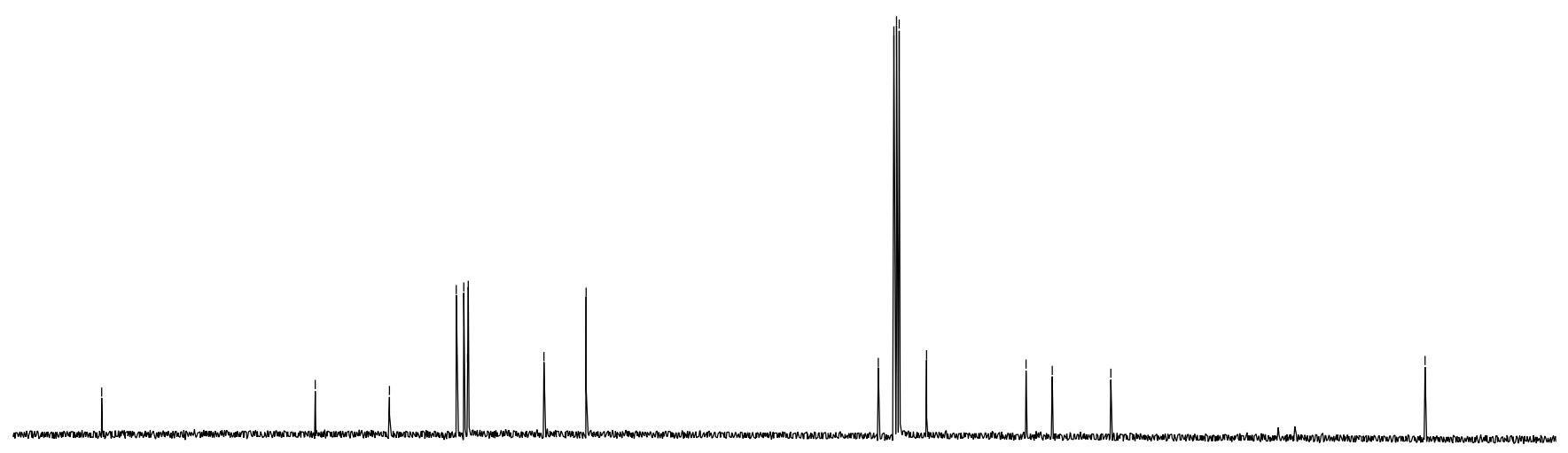




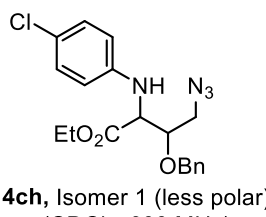

$\left(\mathrm{CDCl}_{3}, 600 \mathrm{MHz}\right)$
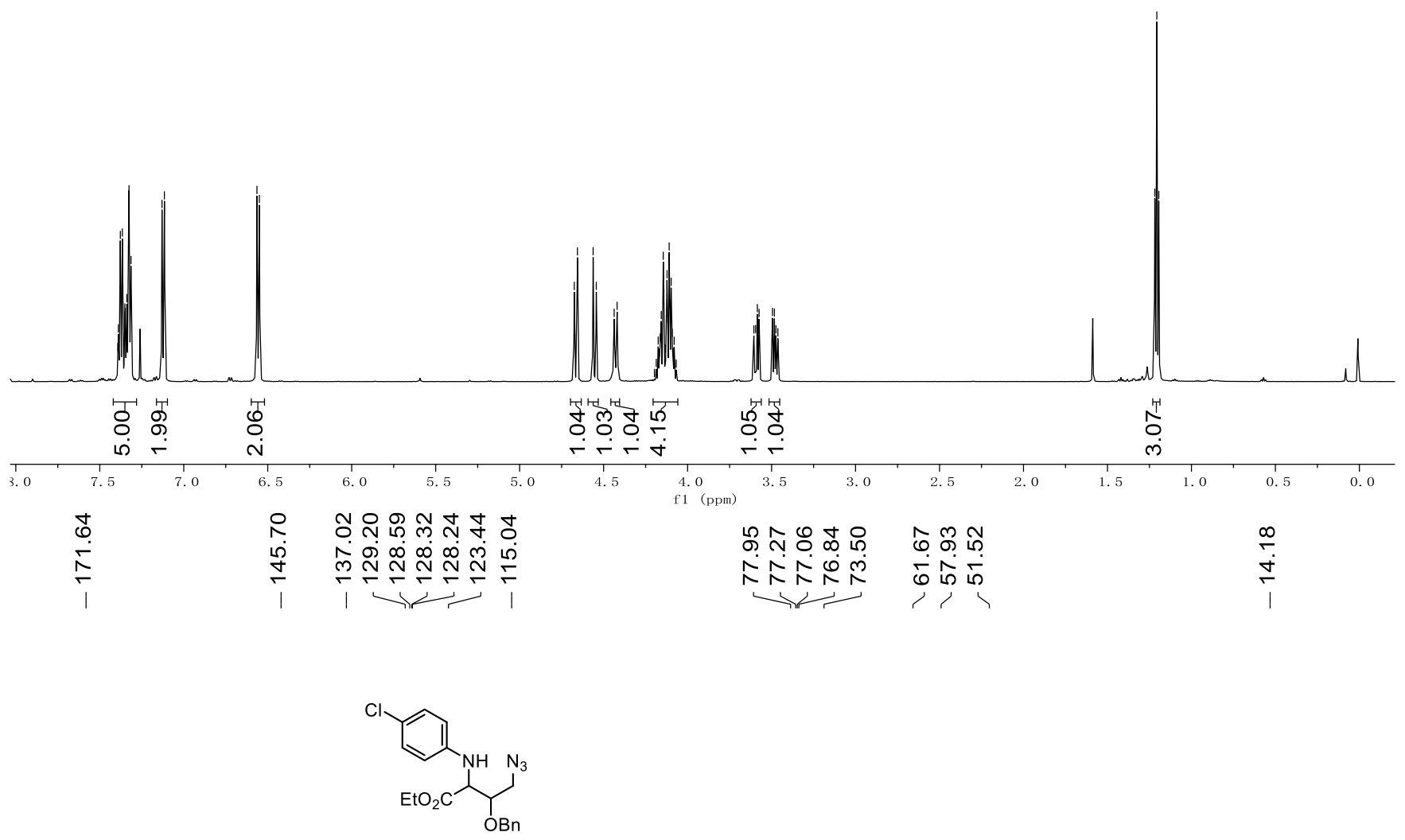

4ch, Isomer 1 (less polar)

$\left(\mathrm{CDCl}_{3}, 151 \mathrm{MHz}\right)$

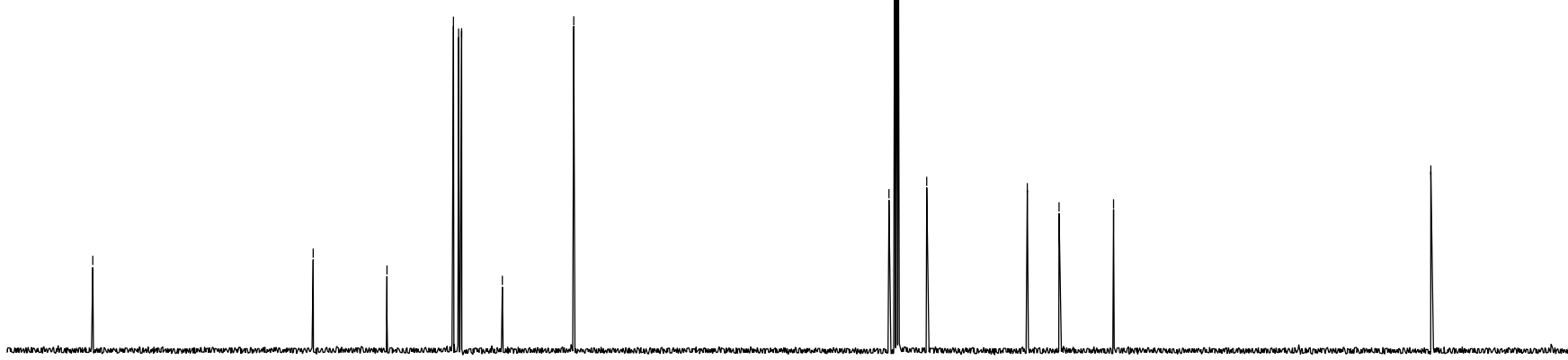

180

$170 \quad 160$

150

90

$80 \quad 70$

60

40

30 


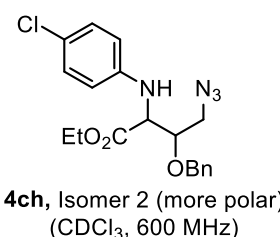

$$
\left(\mathrm{CDCl}_{3}, 600 \mathrm{MHz}\right)
$$

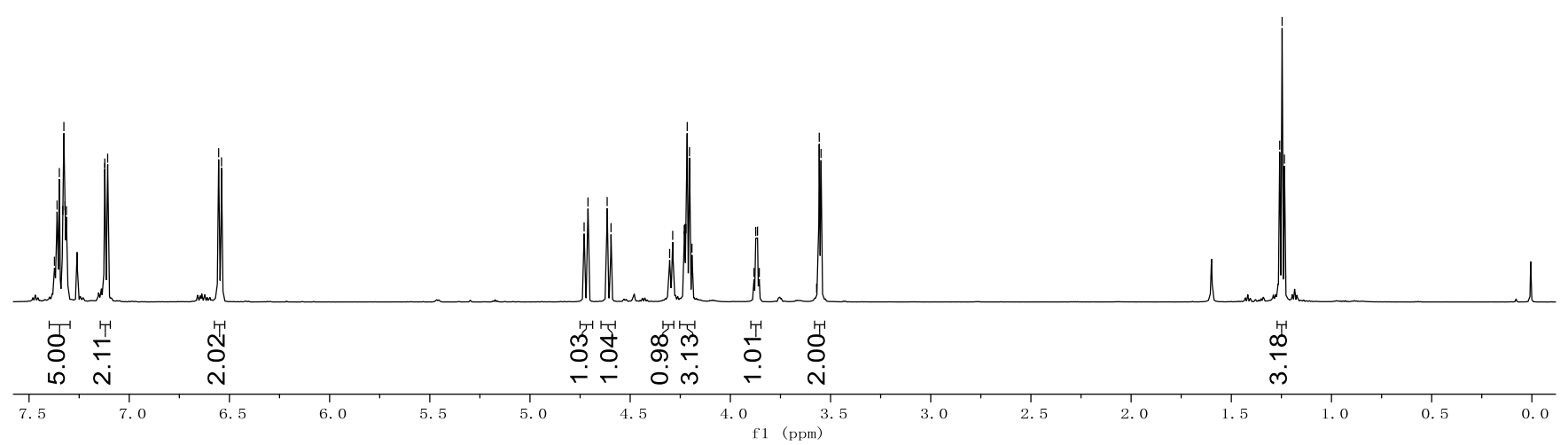

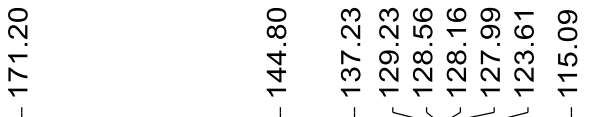

万ฺ

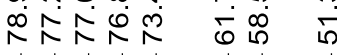

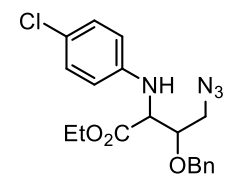

4ch, Isomer 2 (more polar)

$\left(\mathrm{CDCl}_{3}, 151 \mathrm{MHz}\right)$
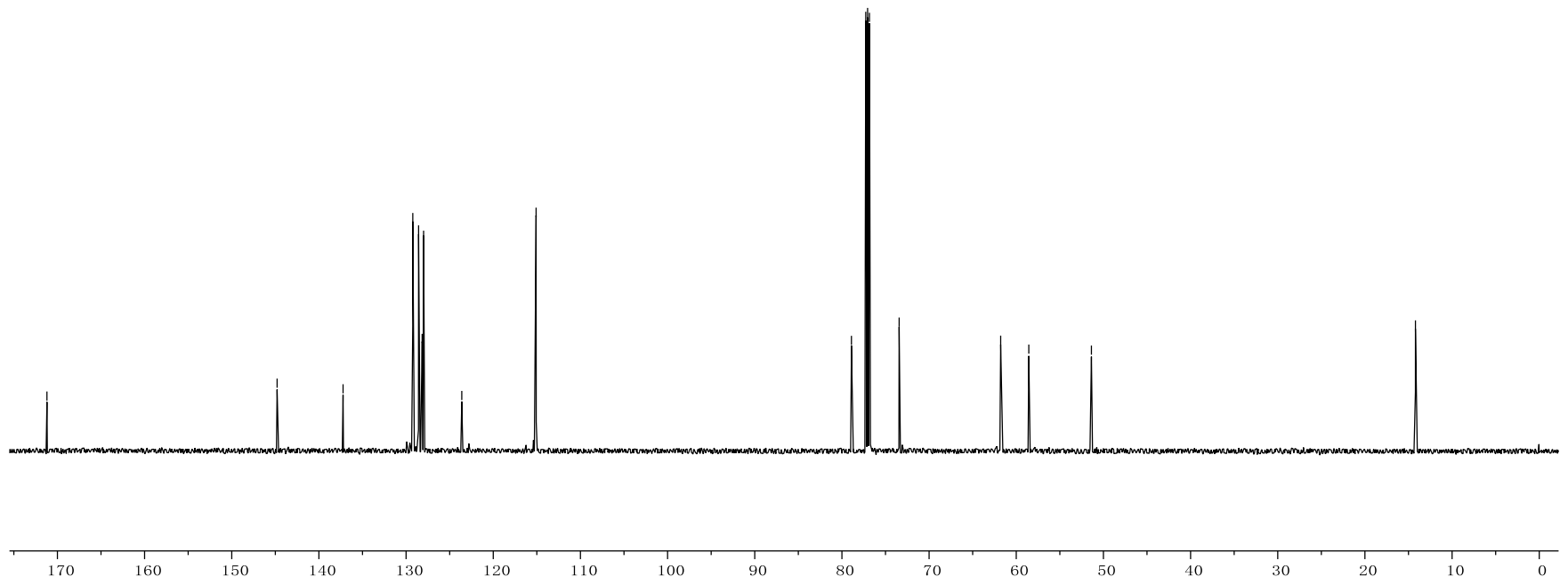

140

130

f1 (ppm) 


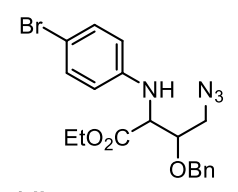

4dh, Isomer 1 (less polar)

$\left(\mathrm{CDCl}_{3}, 400 \mathrm{MHz}\right)$
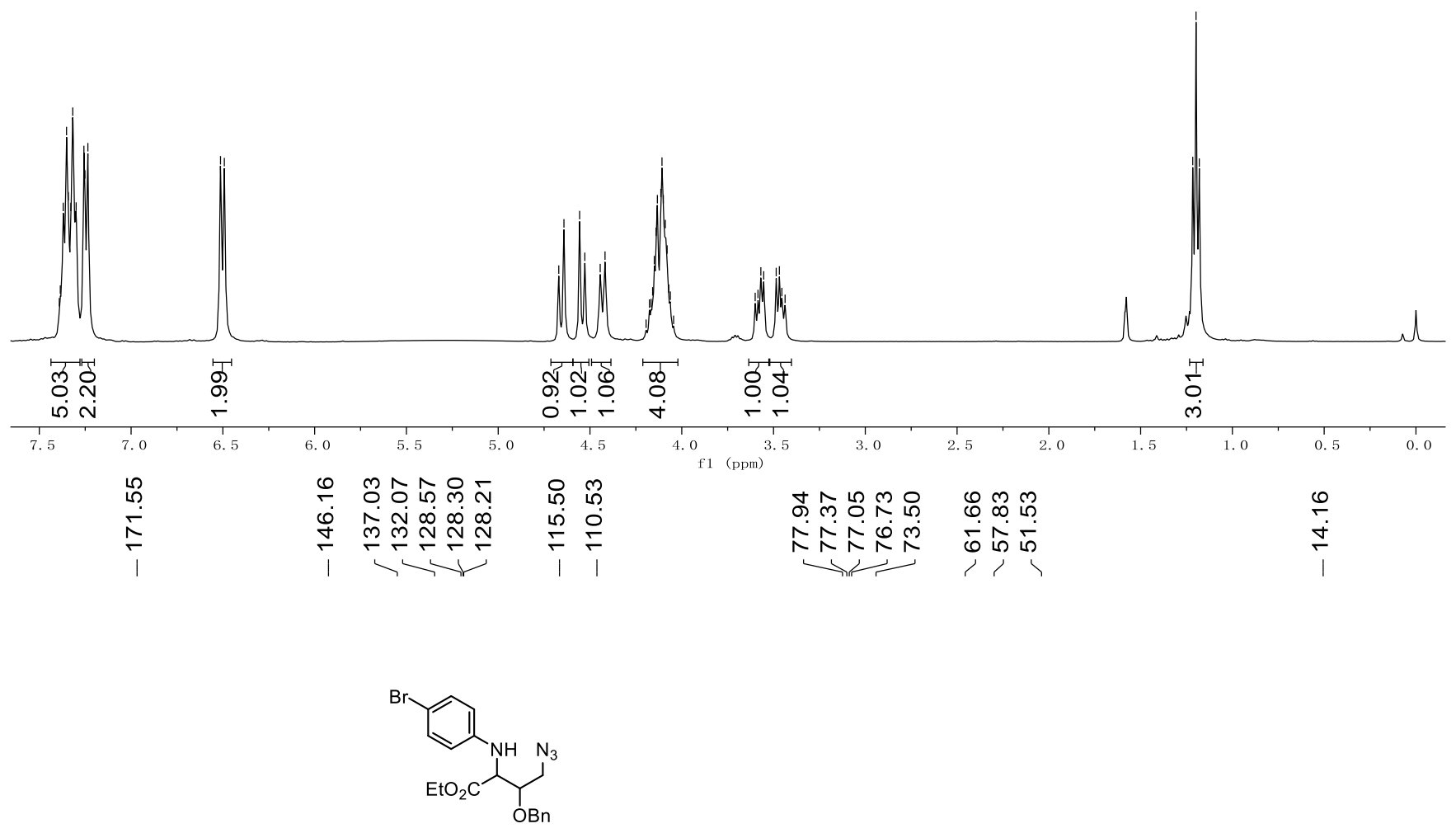

4dh, Isomer 1 (less polar)

$\left(\mathrm{CDCl}_{3}, 101 \mathrm{MHz}\right)$

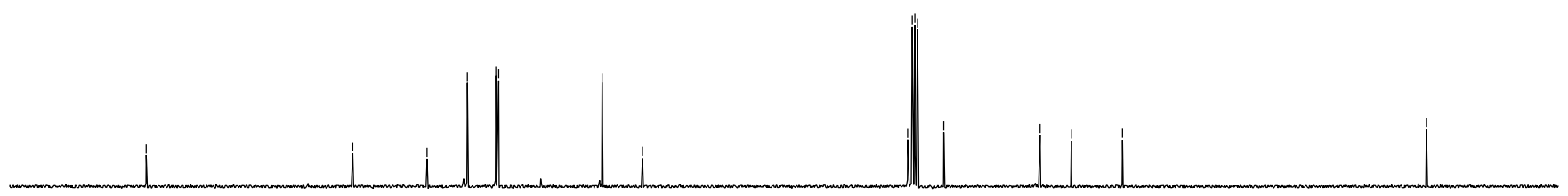




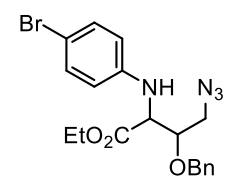

4dh, Isomer 2 (more polar) $\left(\mathrm{CDCl}_{3}, 400 \mathrm{MHz}\right)$
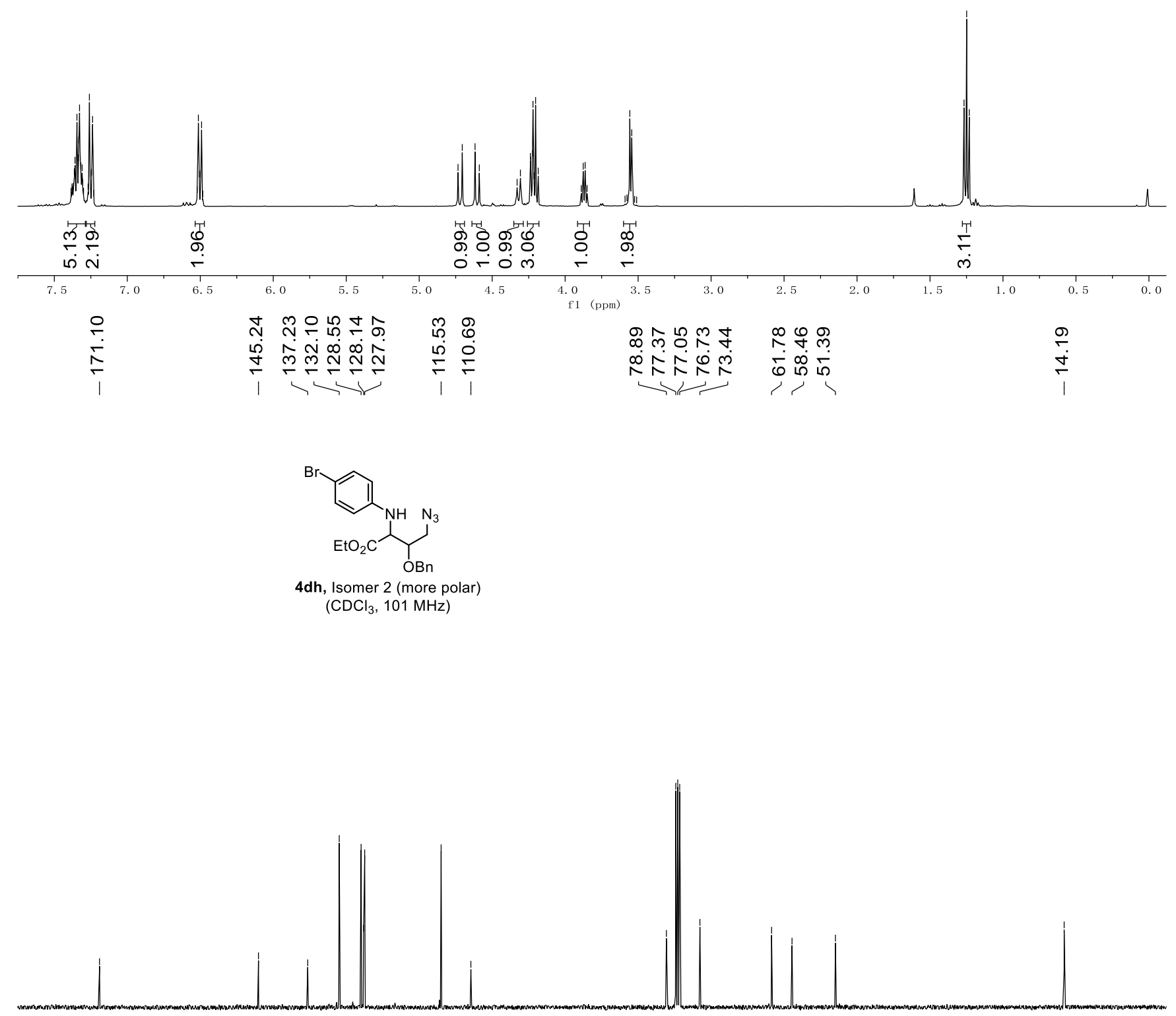

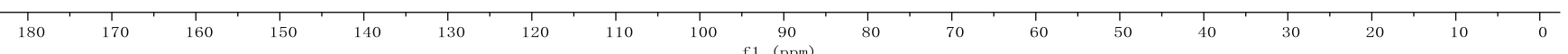




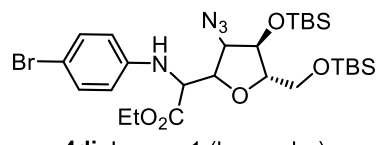

4di, Isomer 1 (less polar)

$\left(\mathrm{CDCl}_{3}, 400 \mathrm{MHz}\right)$
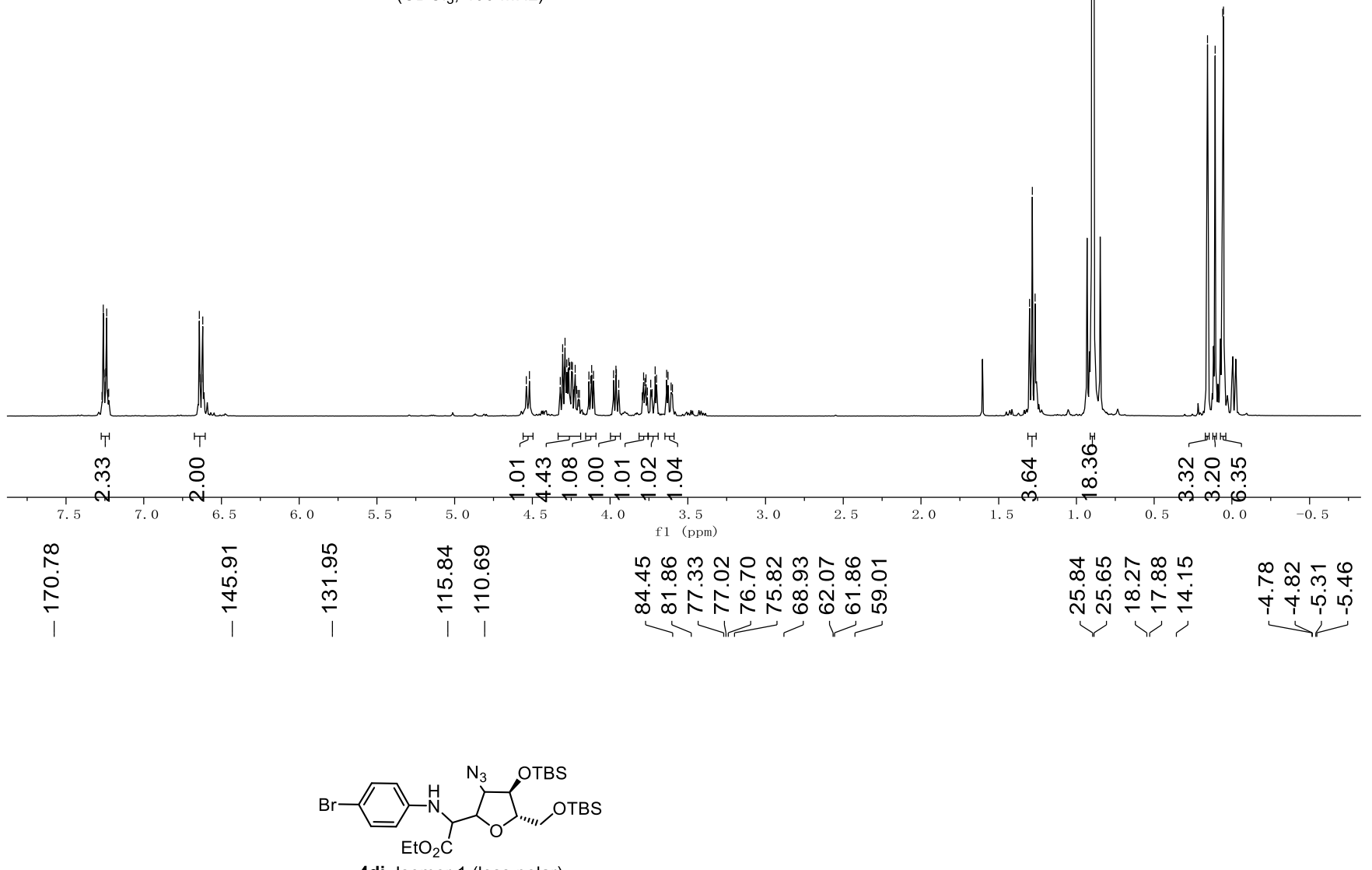

4di, Isomer 1 (less polar)

$\left(\mathrm{CDCl}_{3}, 101 \mathrm{MHz}\right)$

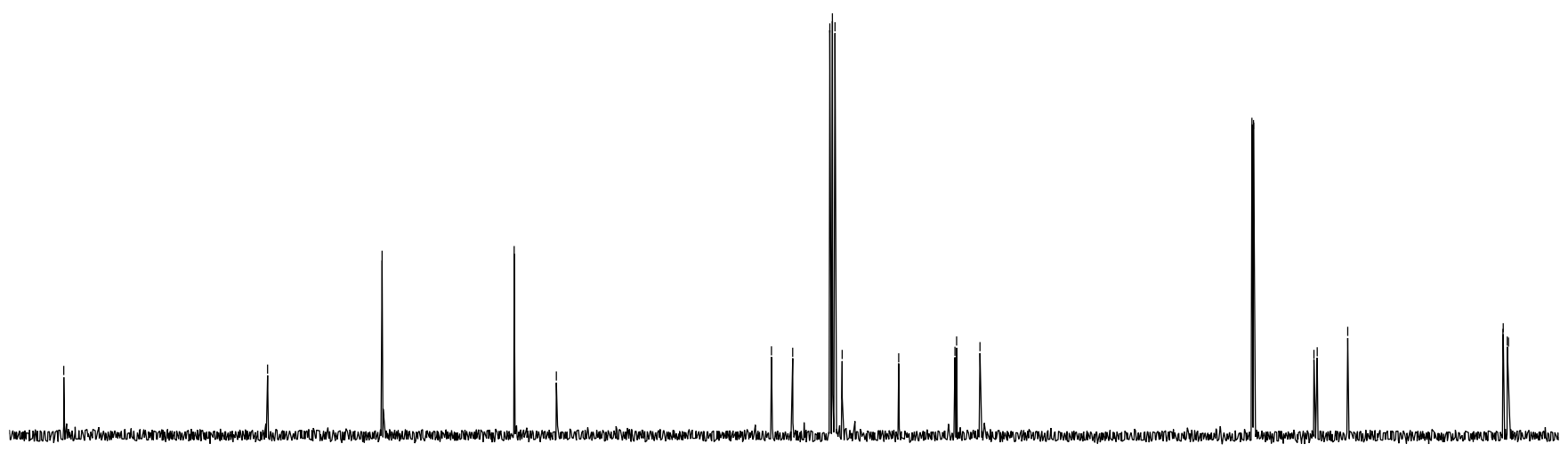

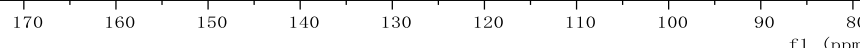




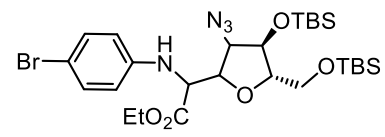

4di, Isomer 2 (more polar)

$\left(\mathrm{CDCl}_{3}, 600 \mathrm{MHz}\right)$

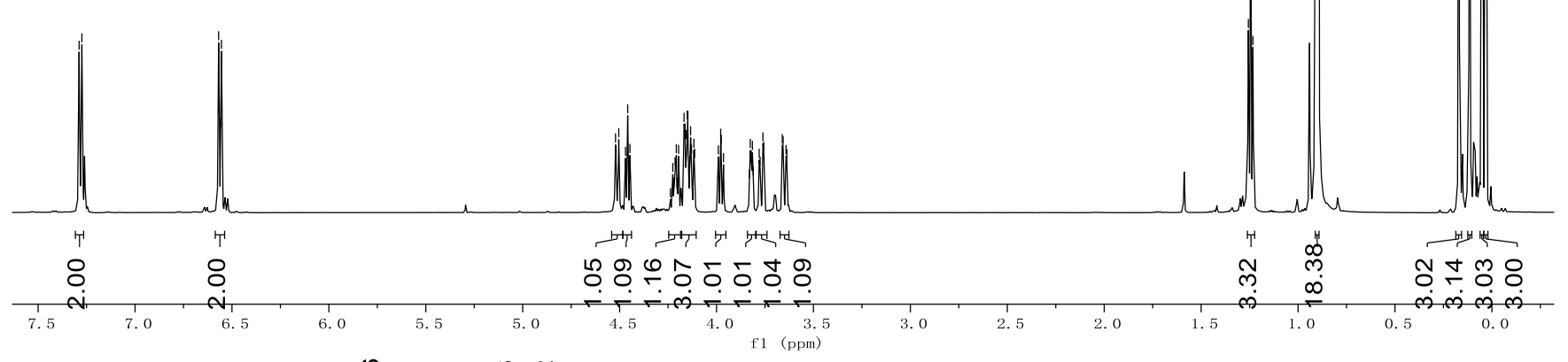

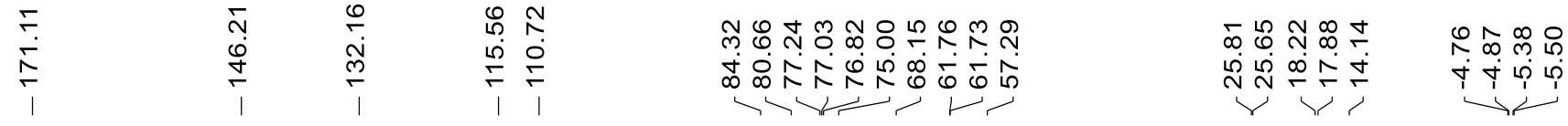

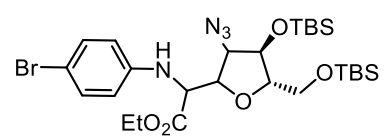

4di, Isomer 2 (more polar)

$\left(\mathrm{CDCl}_{3}, 151 \mathrm{MHz}\right)$

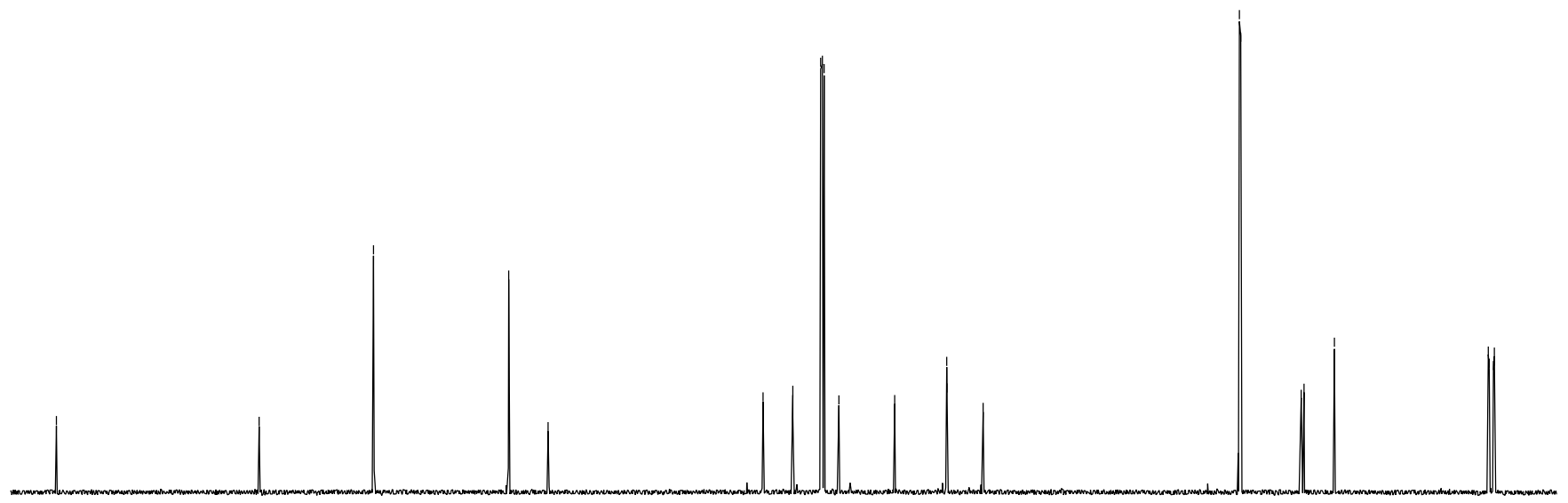

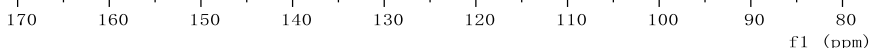




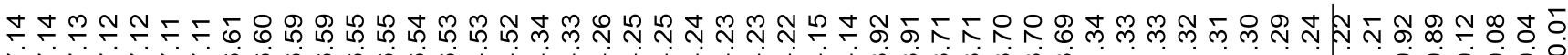

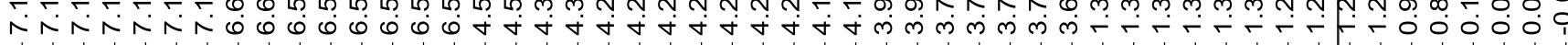

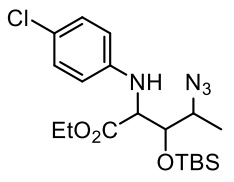

6, Isomer ( $1+2)$

$\left(\mathrm{CDCl}_{3}, 600 \mathrm{MHz}\right)$

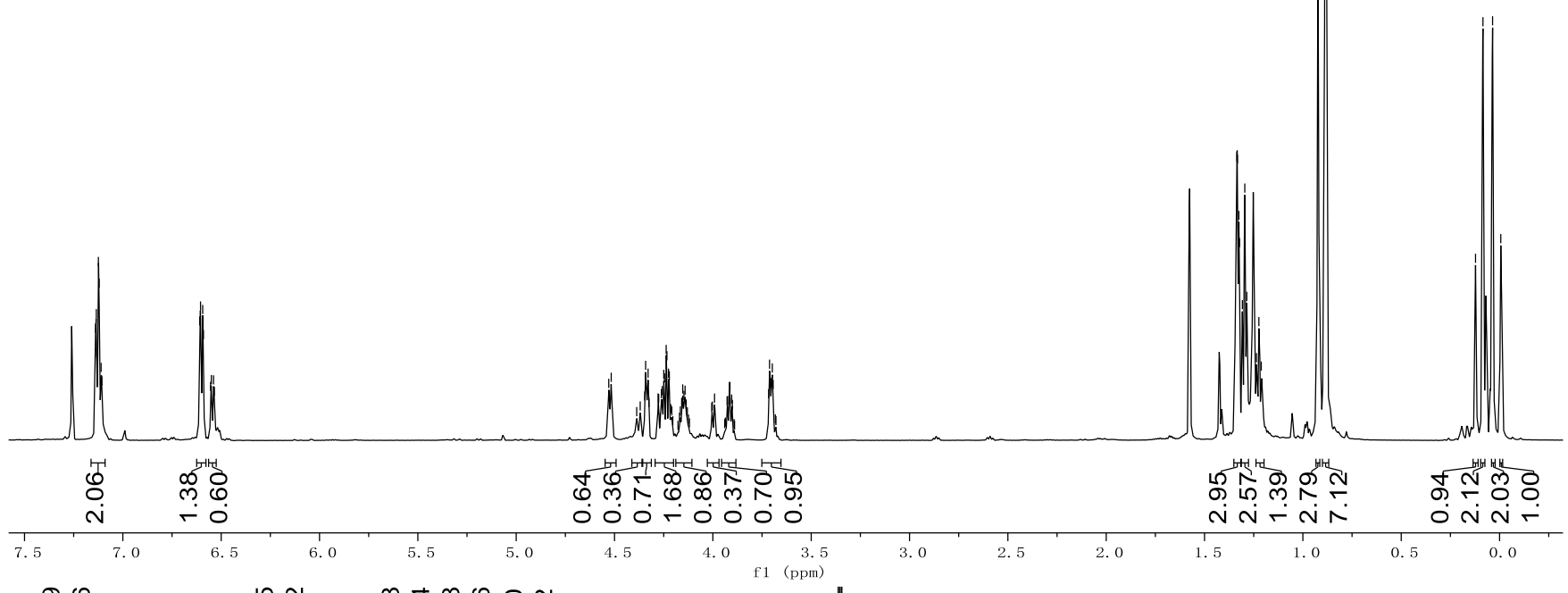

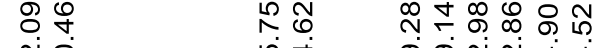

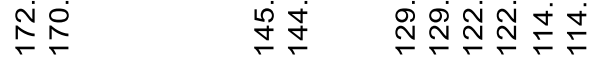

i,

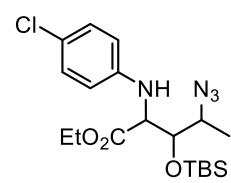

6, Isomer $(1+2)$

$\left(\mathrm{CDCl}_{3}, 151 \mathrm{MHz}\right)$

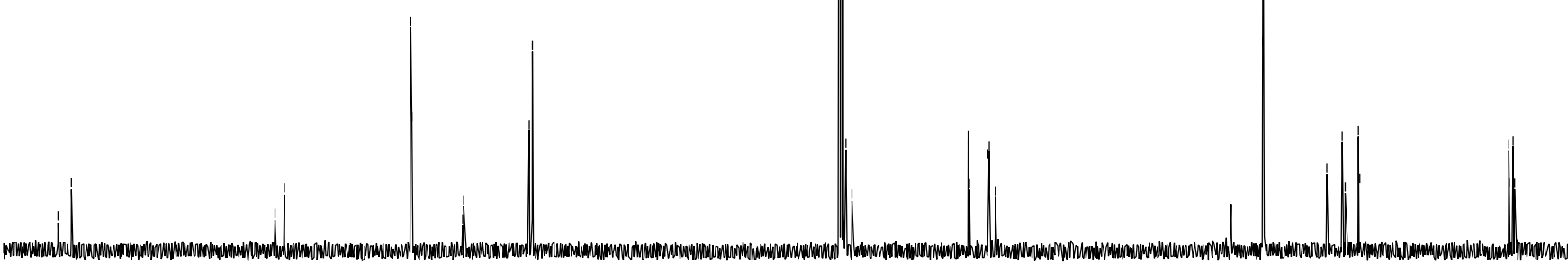

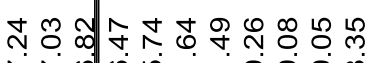

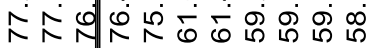

б.

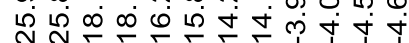

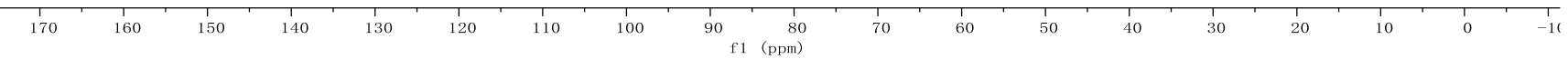




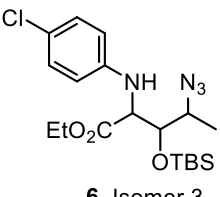

6, Isomer 3

$\left(\mathrm{CDCl}_{3}, 600 \mathrm{MHz}\right)$

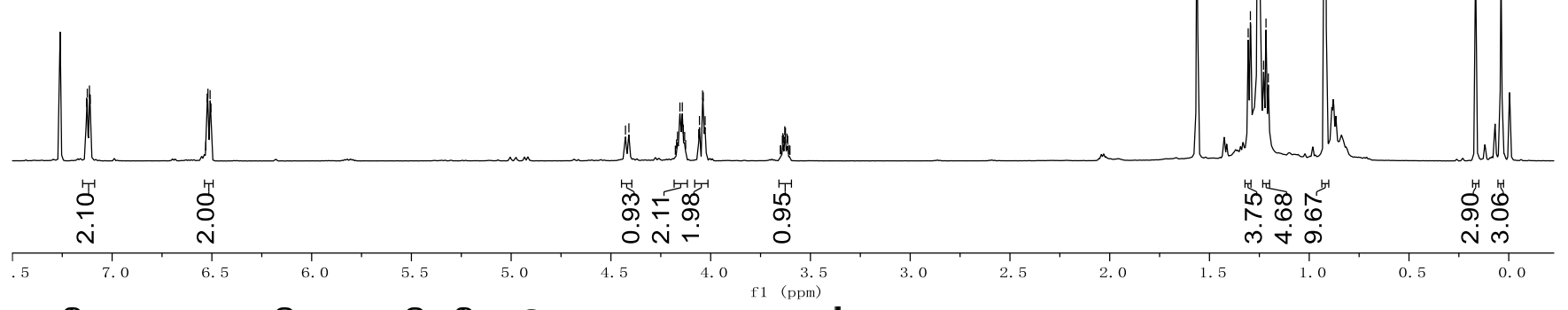

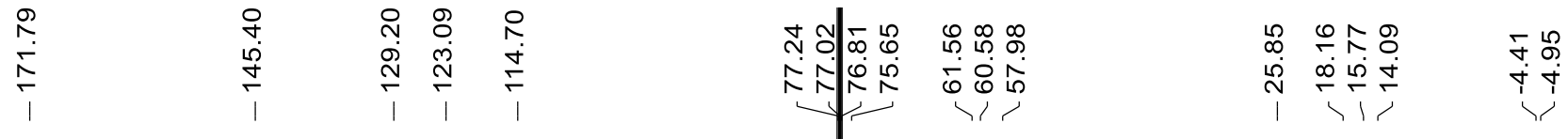
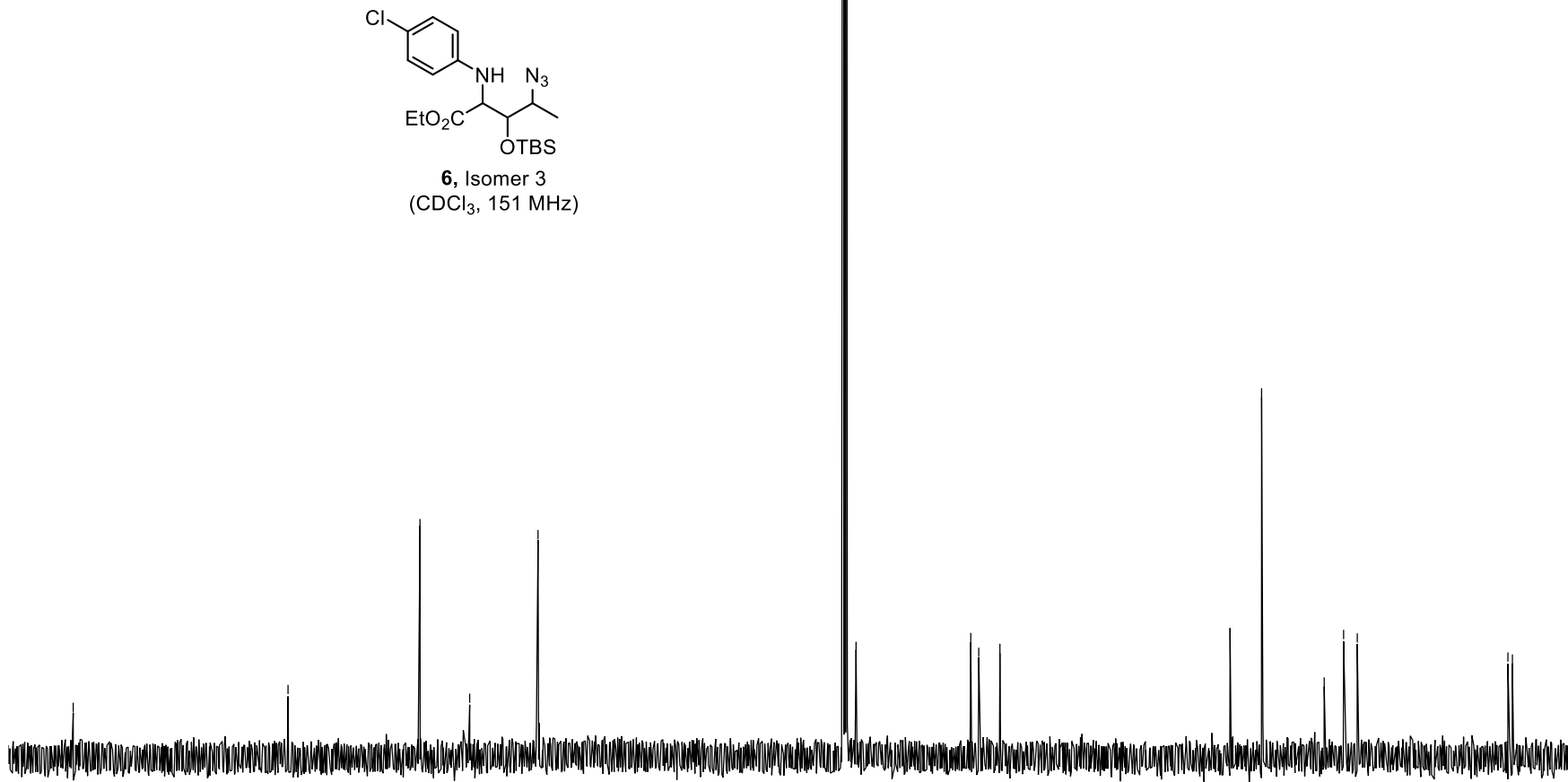

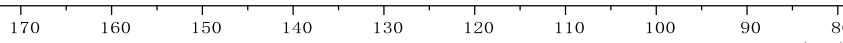



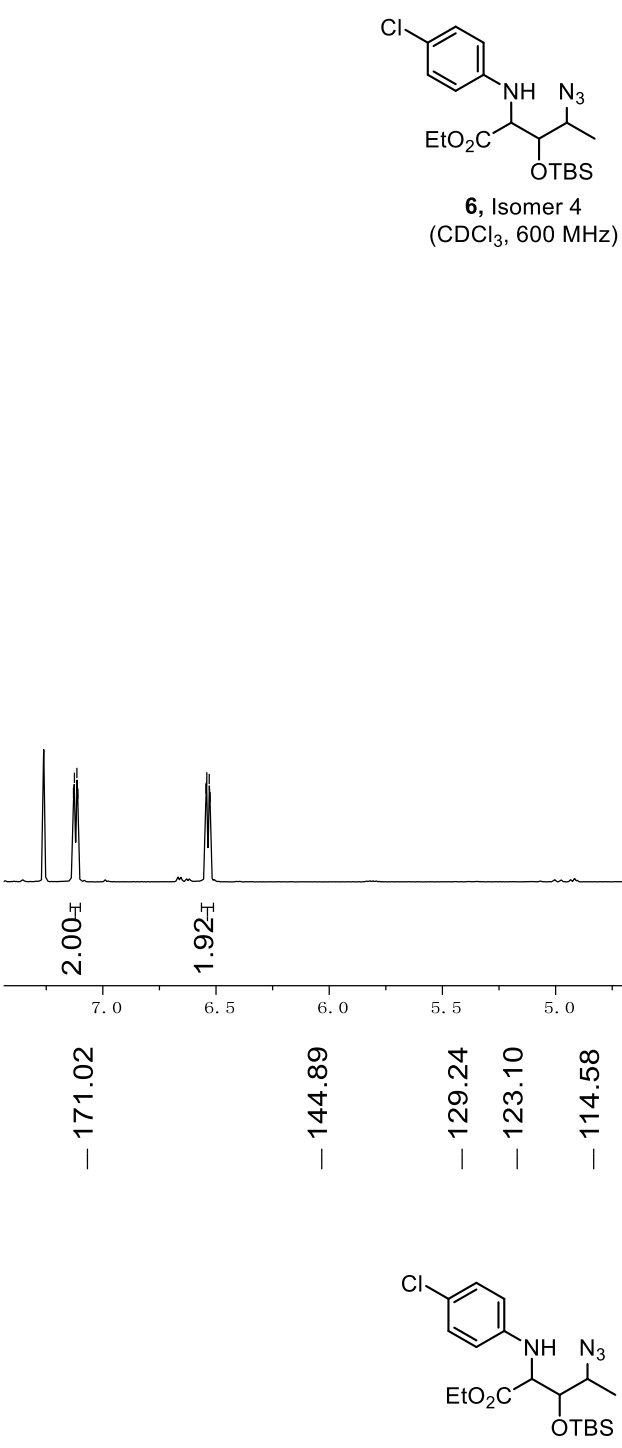

6, Isomer 4 $\left(\mathrm{CDCl}_{3}, 151 \mathrm{MHz}\right)$
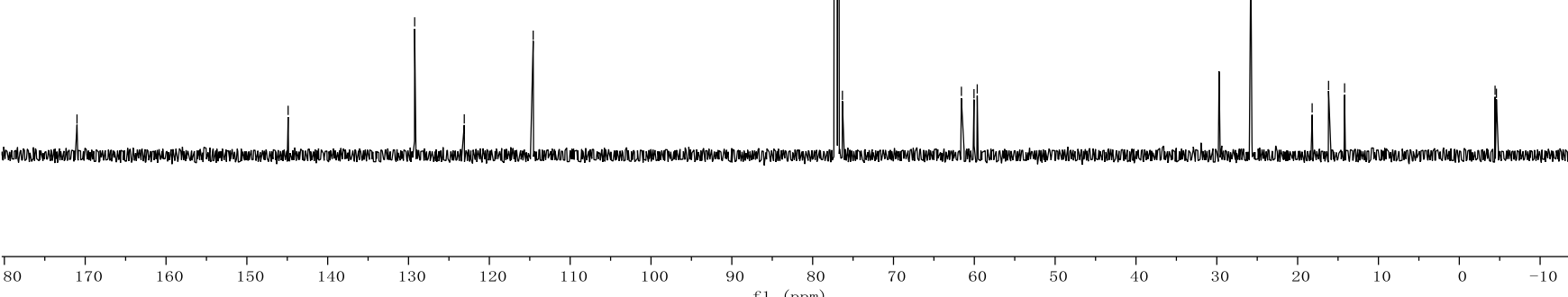


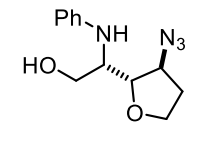

$7\left(\mathrm{CDCl}_{3}, 600 \mathrm{MHz}\right)$
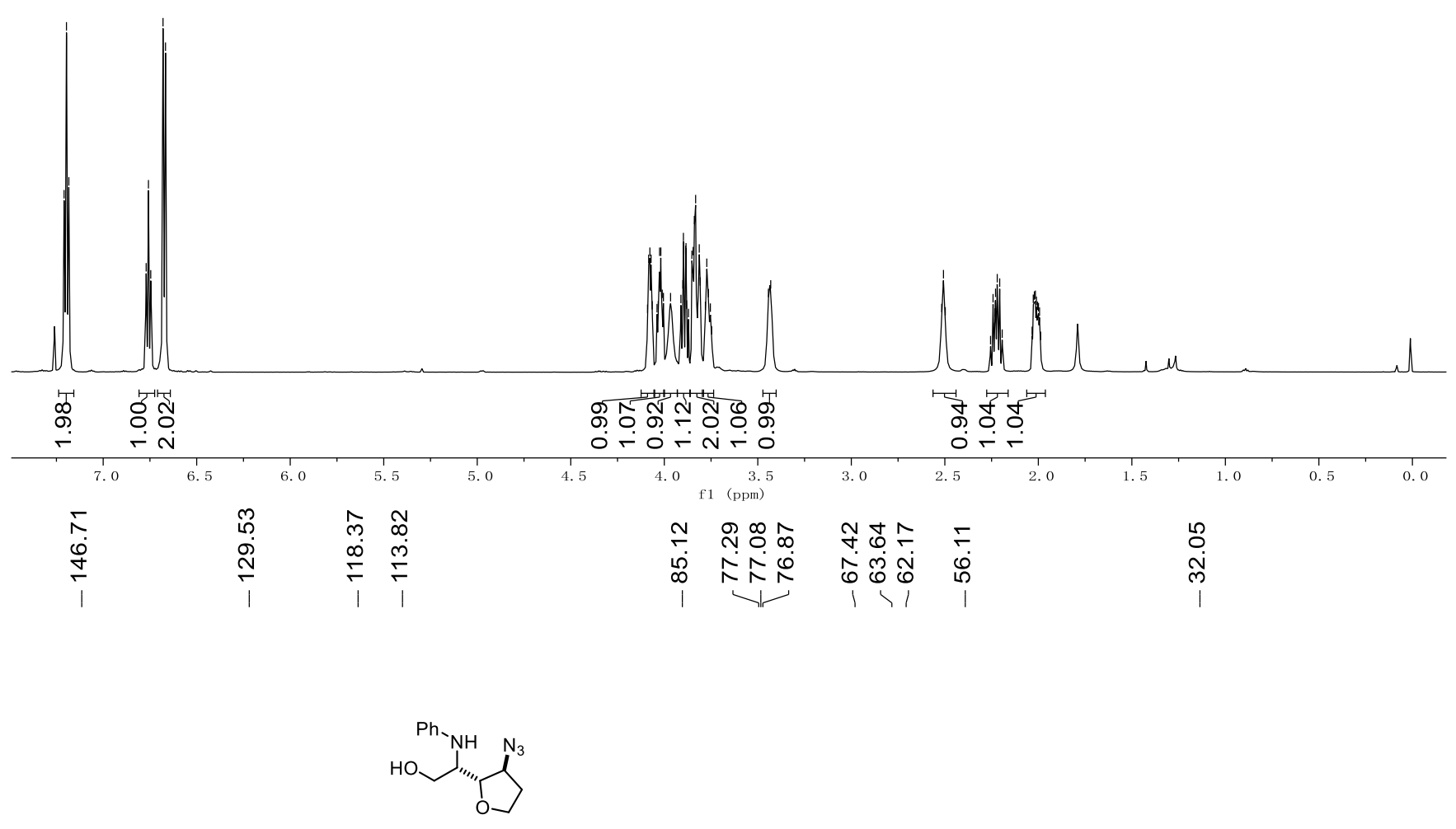

$7\left(\mathrm{CDCl}_{3}, 151 \mathrm{MHz}\right)$

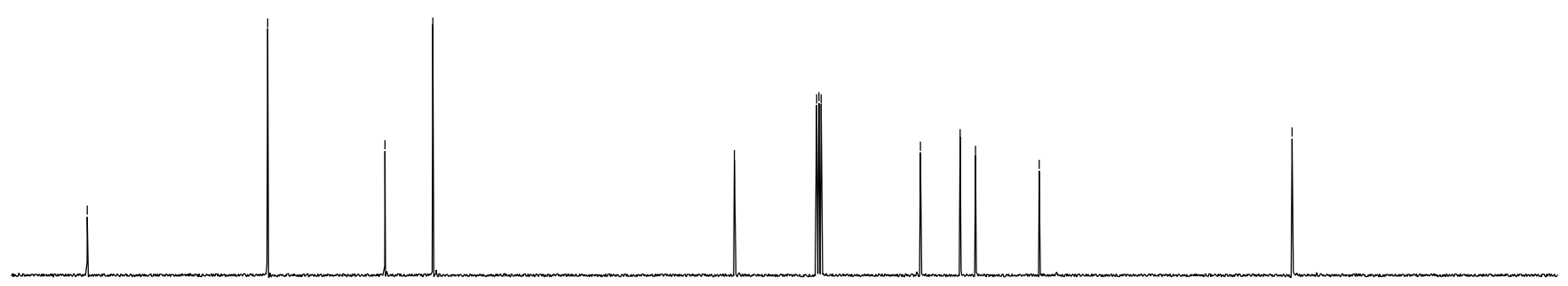

\begin{tabular}{llllllllllllllllllllllllllll|l|l|l|l|l|l|l|l|l|l|l|l}
150 & 145 & 140 & 135 & 130 & 125 & 120 & 115 & 110 & 105 & 100 & 95 & 90 & 85 & 80 & 75 & 70 & 65 & 60 & 55 & 50 & 45 & 40 & 35 & 30 & 25 & 20 & 15 & 10
\end{tabular} 

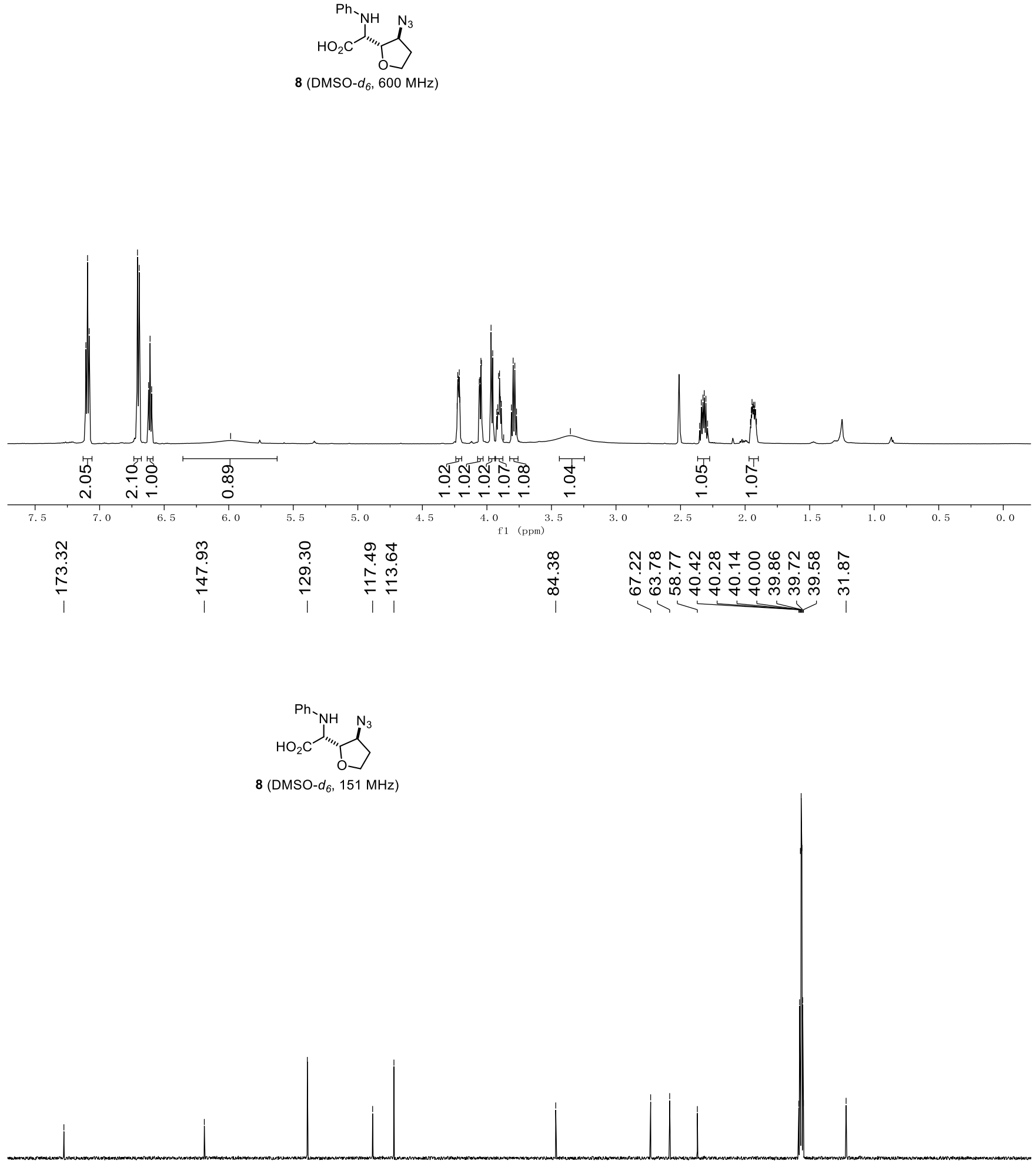

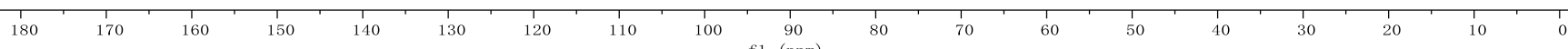



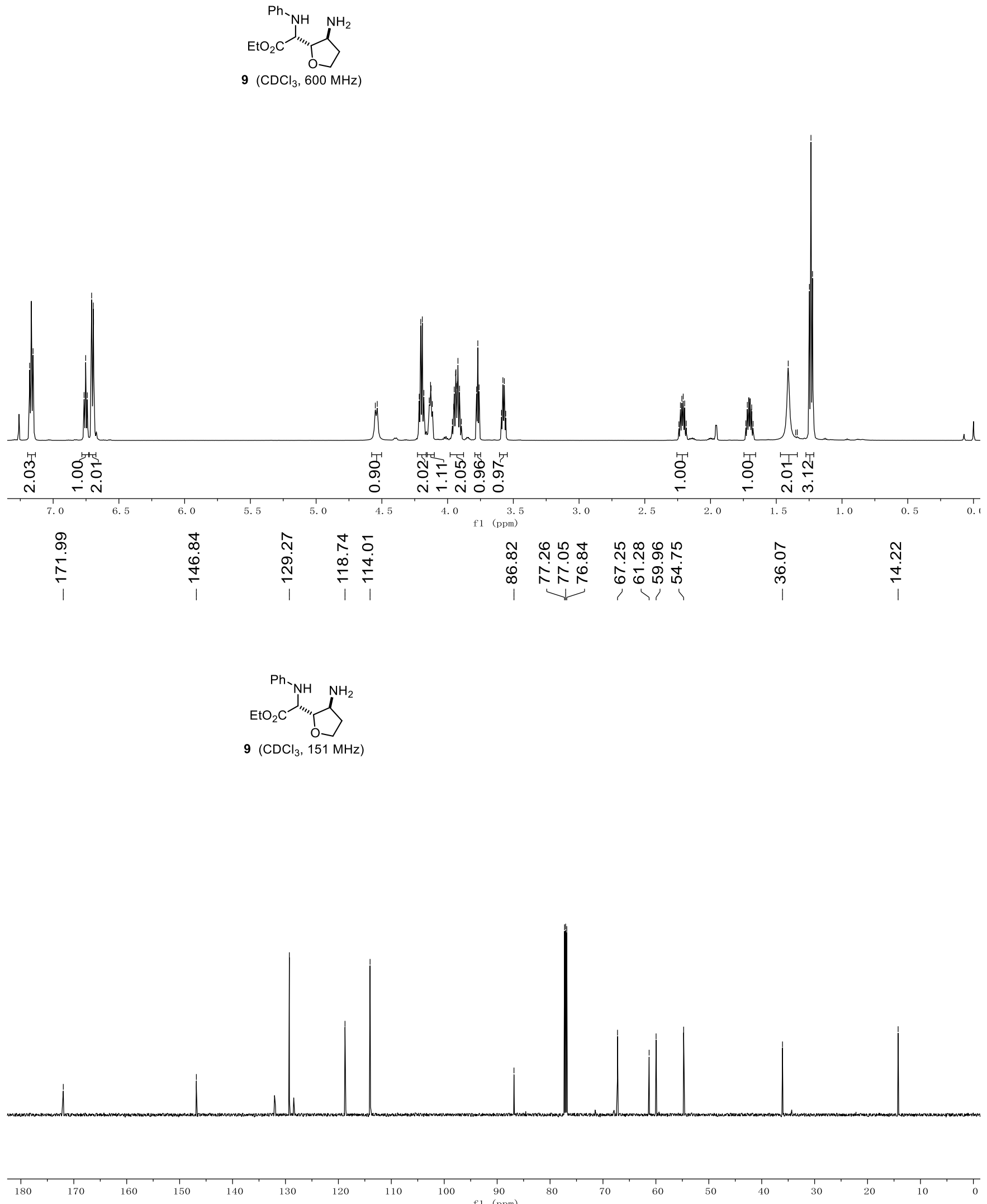

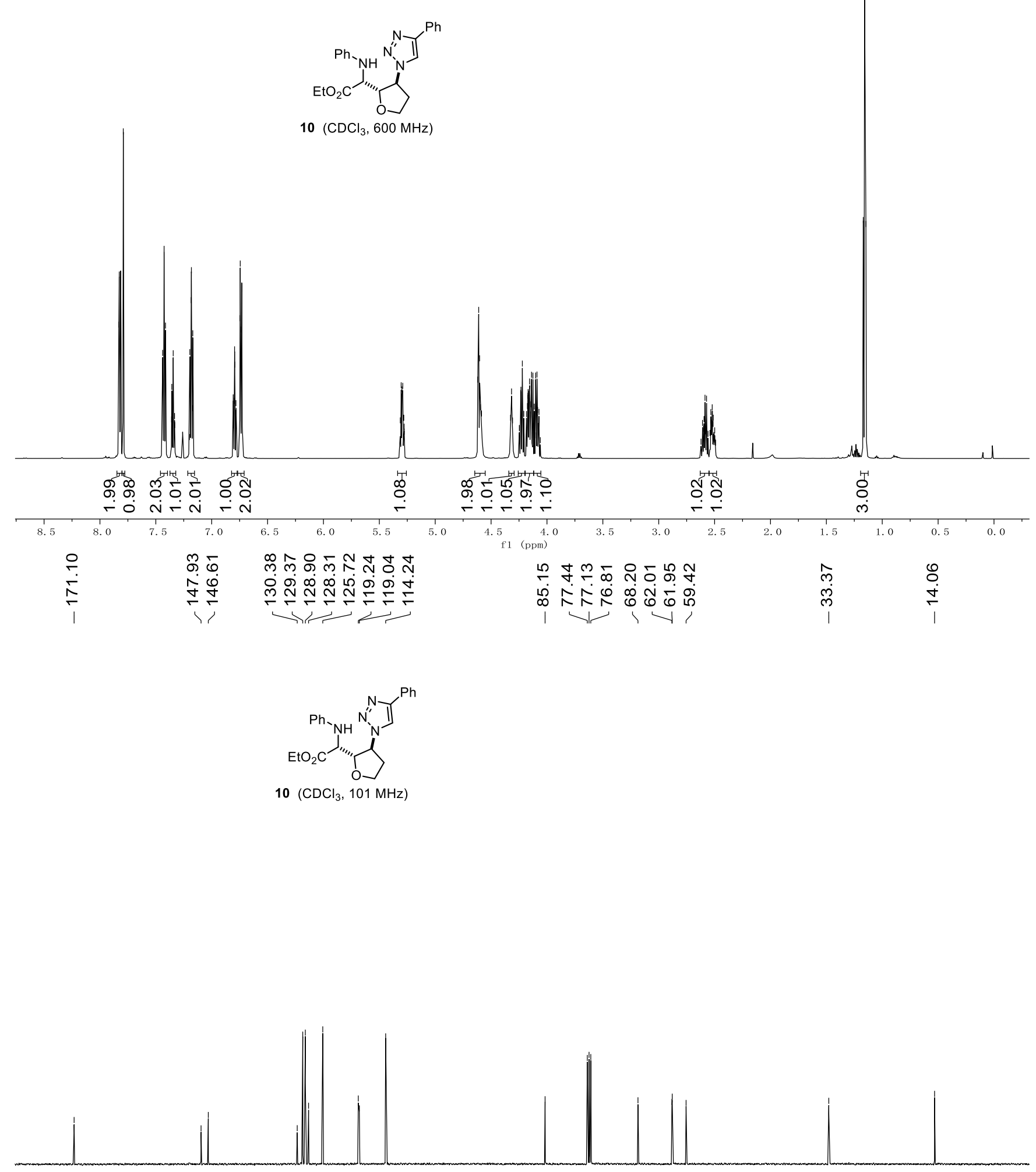

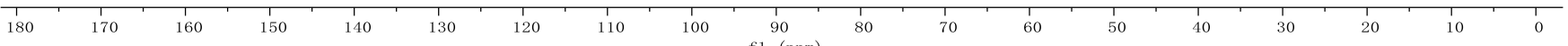




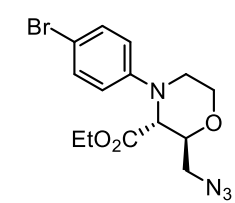

trans-11 $\left(\mathrm{CDCl}_{3}, 600 \mathrm{MHz}\right)$
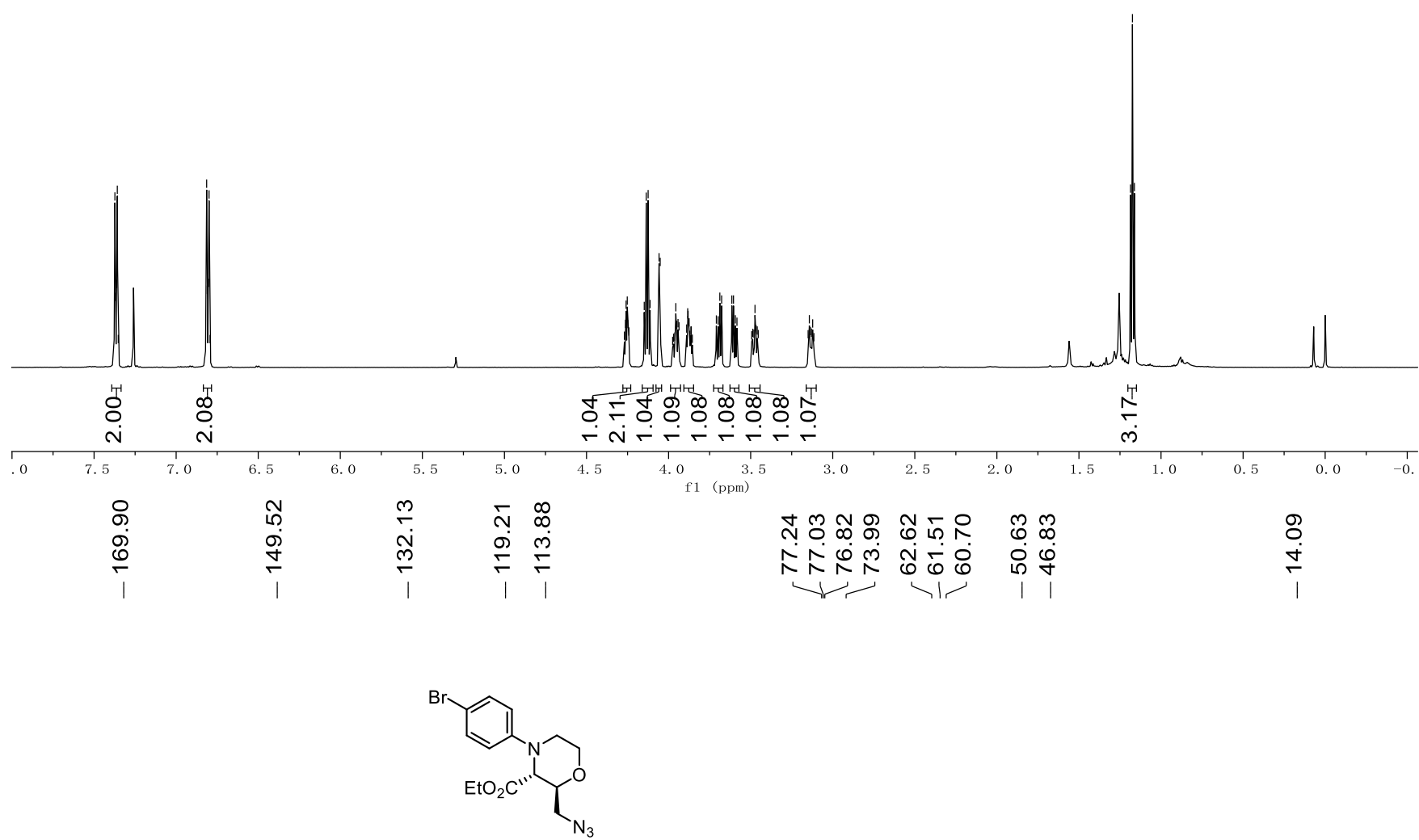

trans-11 $\left(\mathrm{CDCl}_{3}, 151 \mathrm{MHz}\right)$

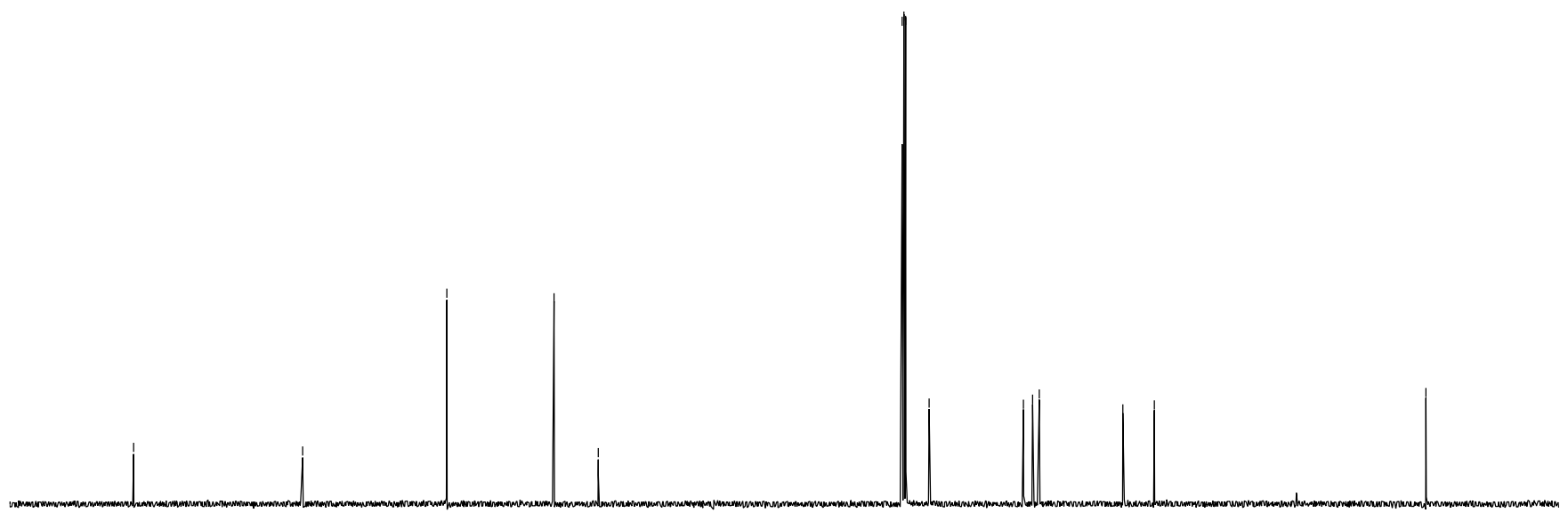




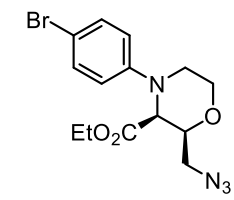

cis-11 $\left(\mathrm{CDCl}_{3}, 400 \mathrm{MHz}\right)$
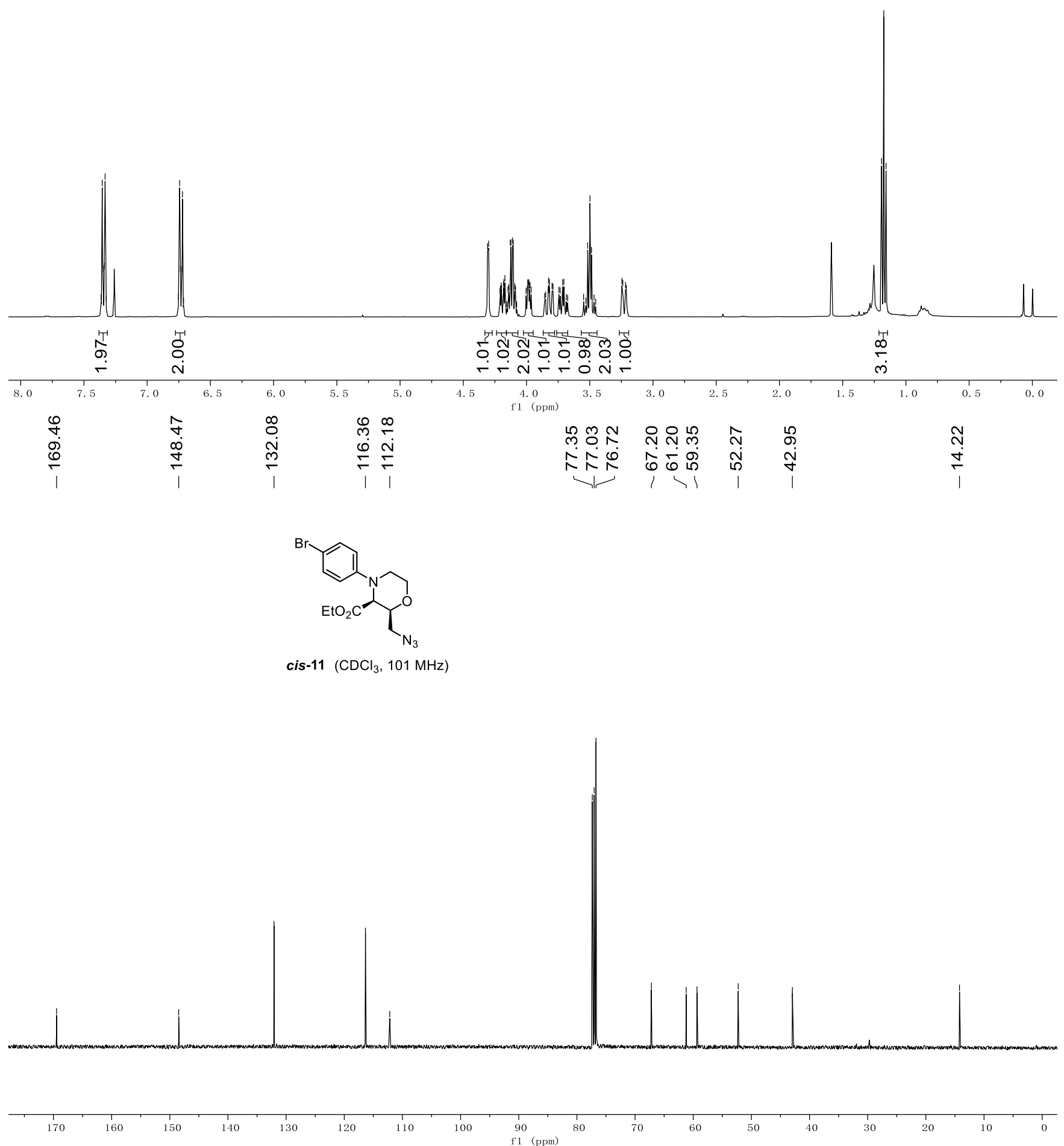


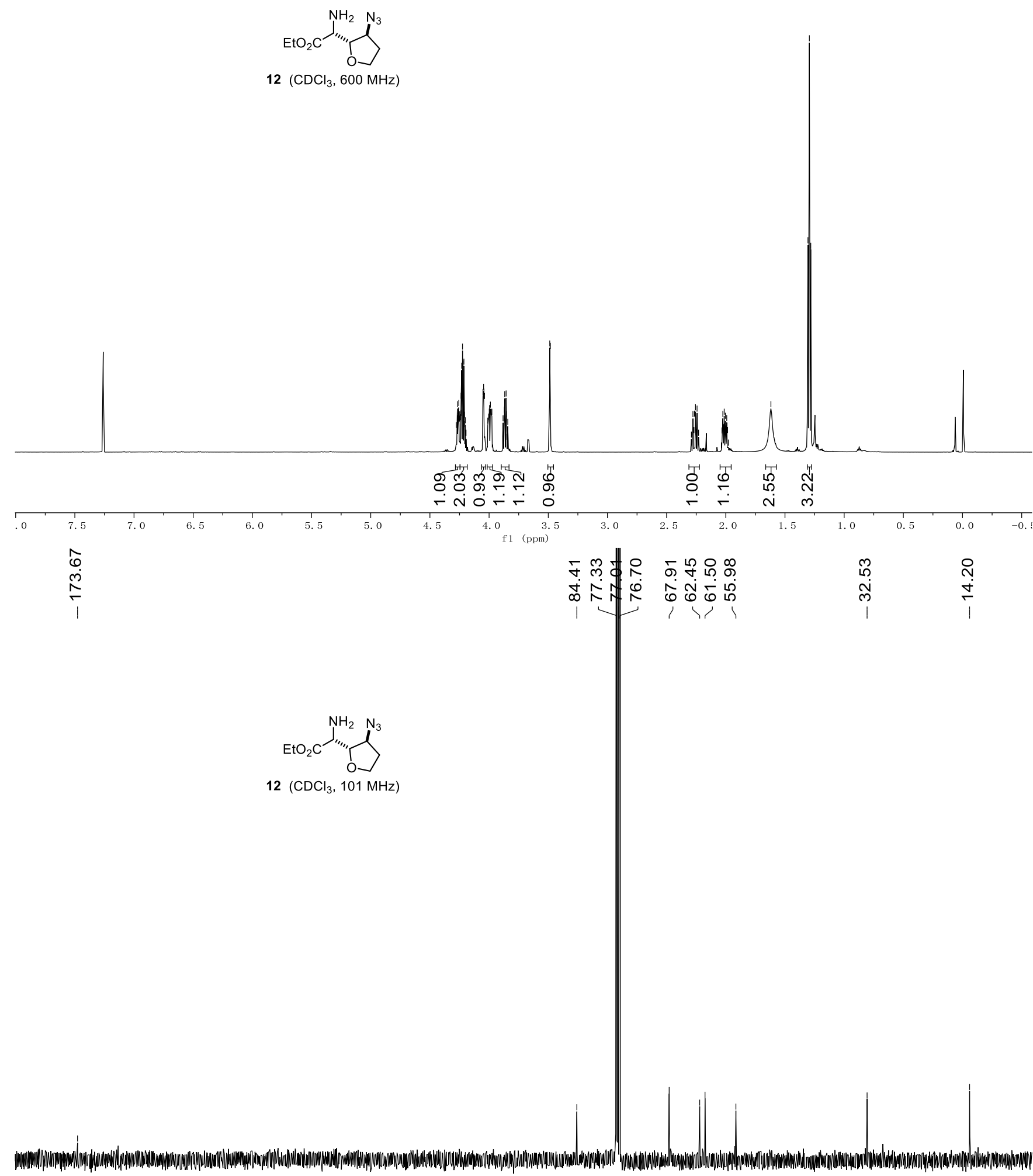

\begin{tabular}{|c|c|c|c|c|c|c|c|c|c|c|c|c|c|c|c|c|}
\hline 180 & 170 & 160 & 150 & 140 & 130 & 120 & 110 & 100 & 90 & 80 & 70 & 60 & 50 & 40 & 30 & 20 \\
\hline
\end{tabular}




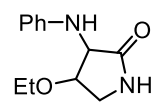

$13\left(\mathrm{CDCl}_{3}, 400 \mathrm{MHz}\right)$
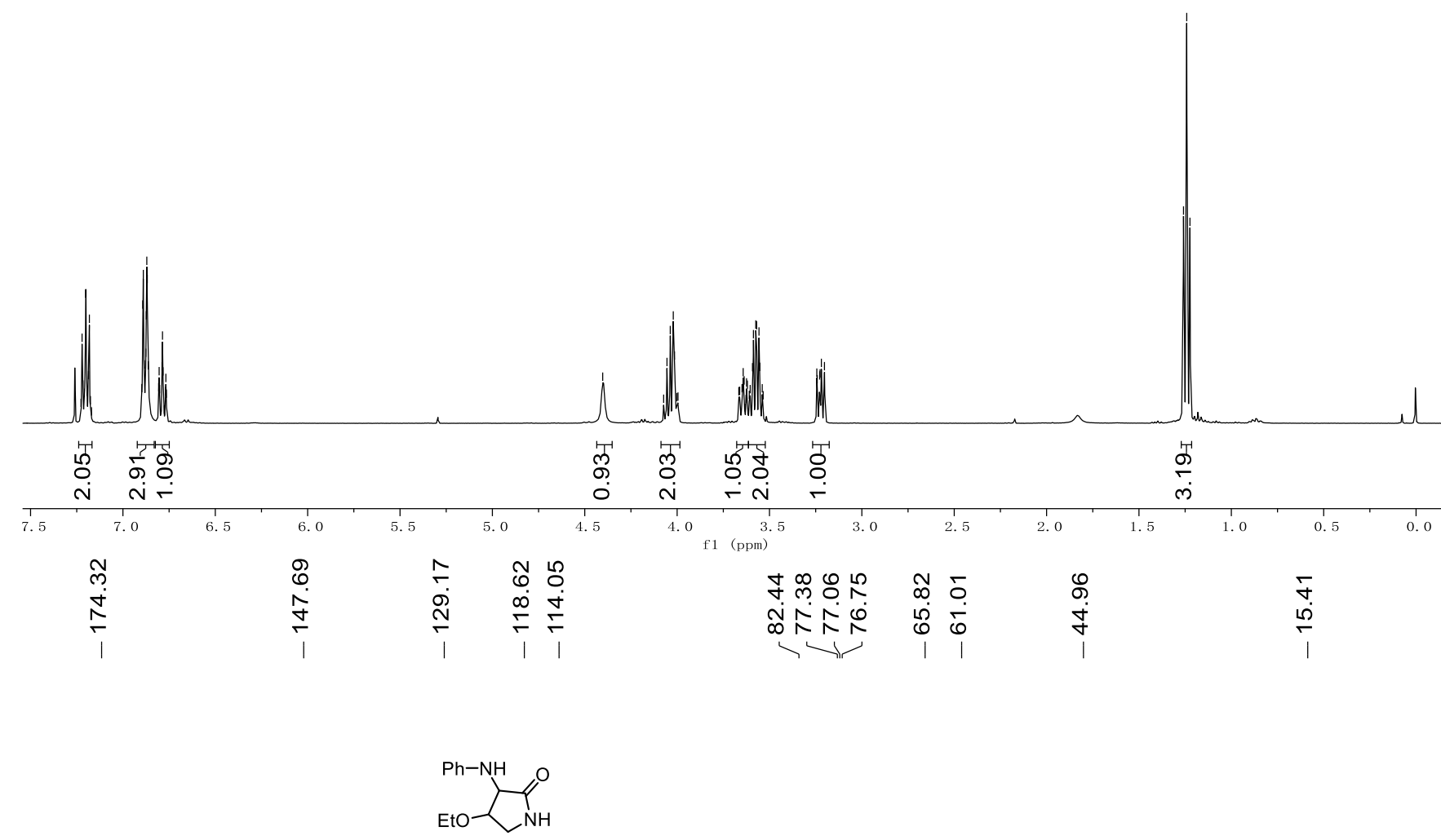

$13\left(\mathrm{CDCl}_{3}, 101 \mathrm{MHz}\right)$
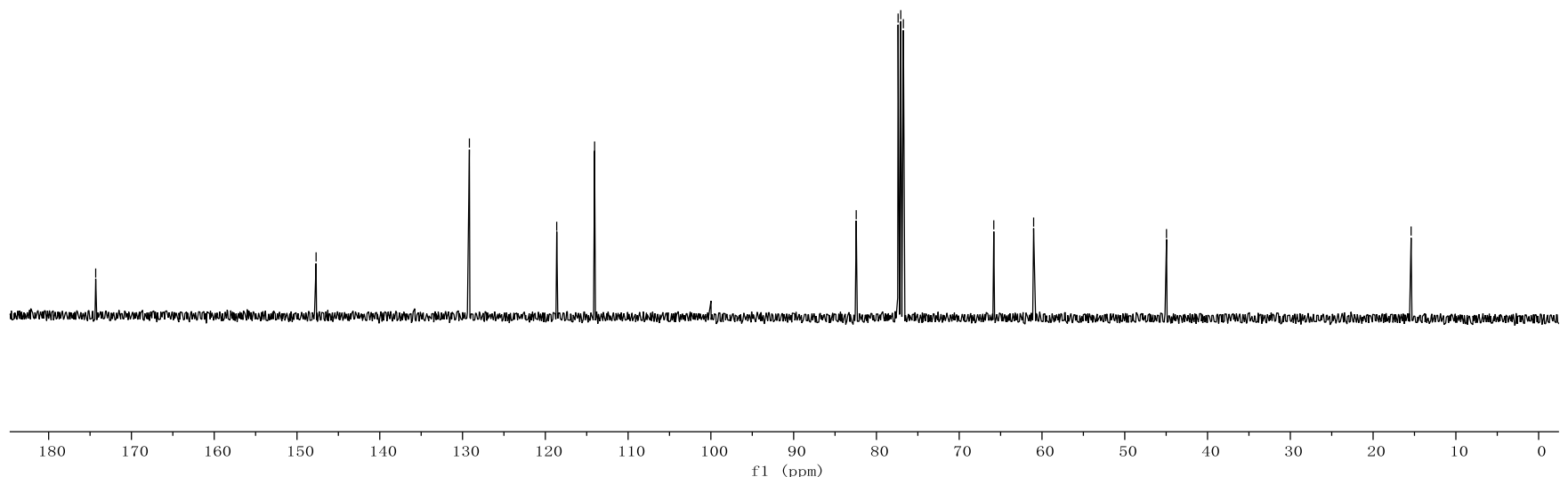